\title{
LECTURES ON REPRESENTATIONS of Surface Groups
}

\author{
Notes of a course given in \\ ETH-Zürich Fall 2006, \\ and \\ Orsay Spring 2007-2008.
}

\section{François LABOURIE ${ }^{1}$}

(Based on notes taken by Tobias HARTNICK)

April 12, 2011

\footnotetext{
${ }^{1}$ Univ. Paris-Sud, Laboratoire de Mathématiques, Orsay F-91405 Cedex; CNRS, Orsay cedex, F-91405, The research leading to these results has received funding from the European Research Council under the European Community's seventh Framework Programme (FP7/2007-2013)/ERC grant agreement $\mathrm{n}^{\circ}$ FP7-246918, as well as well as from the ANR program ETTT (ANR-09-BLAN-0116-01)
} 


\section{Chapter 1}

\section{Introduction}

The purpose of this lecture series is to describe the representation variety - or character variety - of the fundamental group $\pi_{1}(S)$ of a closed connected surface $S$ of genus greater than 2 , with values in a Lie group $G$. This character variety is roughly defined to be

$$
\operatorname{Rep}\left(\pi_{1}(S), G\right):=\operatorname{Hom}\left(\pi_{1}(S), G\right) / G,
$$

where $G$ acts on $\operatorname{Hom}\left(\pi_{1}(S), G\right)$ by conjugation.

These character varieties have been heavily studied in the context of gauge theories - mainly in the case of compact groups - and hyperbolic geometry when $G=\operatorname{PSL}(2, \mathbb{R})$ or $G=\operatorname{PSL}(2, \mathbb{C})$.

We shall mainly be interested in these lectures on the topology of these varieties and their symplectic structure which was discovered by Atiyah-BottGoldman, setting aside their interpretation in the theory of Riemann surfaces.

In the preliminary chapters, we give a crash course on surfaces, vector bundles and connections. While there is no new material here, we insist on describing very early both differential geometric and combinatorial aspects of the objects that we are interested in.

These various point of view allow us to give several models of the character variety generalising the familiar picture about first cohomology groups being described alternatively using de Rham, Čech or simplicial cohomology or as the space of homomorphisms of the fundamental group into $\mathbb{R}$.

We then describe the smooth structure of these character varieties as well as their tangent spaces. The understanding of the volume form on these tangent spaces requires us, following Witten, to introduce Reidemeister torsion.

When $G=\operatorname{PSL}(2, \mathbb{R})$, we prove Milnor-Wood Inequality and discuss connected components of the character variety.

We finally introduce the symplectic structure on these character varieties and prove Witten's formula which compute their symplectic volume in the case 
of a compact groups. We introduce two important algebras of observables, the first one consisting of Wilson loops or the other of spin networks and compute their Poisson bracket, thus introducing Goldman algebra.

In the last chapter, we turn to the integrality of the symplectic form and the relation with 3-manifolds and the Chern-Simons invariant.

Each chapter is closed by a section giving general references and further readings.

These notes correspond to lectures given at ETH-Zürich and Orsay at a beginning graduate level. The students were supposed to have only elementary knowledge of differential geometry and topology. It follows that these notes are quite informal: in the first chapters, we do not insist in the details of the differential geometric constructions and refer to classical textbooks, while in the more advanced chapters, we are sometimes reduced to give proofs only in special cases hoping that it gives the flavor of the general proofs. A typical example is the study of the properness of the action by conjugation of $G$ on $\operatorname{Hom}\left(\pi_{1}(S), G\right)$ : while the general case requires a certain knowledge of algebraic groups and their action on algebraic varieties, we only treat here the case of $G=\operatorname{SL}(n, \mathbb{R})$ resorting to elementary arguments on matrices.

None of the results presented here are new, but the presentation and proofs sometimes differ from other exposition.

Tobias Hartnick had the difficult task of turning the chaos of my lectures in some decent mathematics, and I heartily thank him for writing up the first draft of these notes, giving them much of their shape and consistence.

I would also like to thank the mathematics department of ETH and the Institute for Mathematical Research (FIM) in Zürich for their invitation.

These notes would certainly not have existed without the comments and encouragement of François Laudenbach, who taught me symplectic geometry a long time ago.

I would also like to thank M. Burger, I. Chatterji, W. Goldman, O. GarcíaPrada, S. Kerckhoff, N. Hitchin, A. Iozzi, J. Souto, D. Toledo, R. Wentworth for many helpful comments on the text or the references. 


\section{Contents}

1 Introduction 1

2 Surfaces $\quad 6$

2.1 Surfaces as 2-dimensional manifolds . . . . . . . . . . . . . . 6

2.1.1 Basic definitions . . . . . . . . . . . . . . 6

2.1.2 Surfaces with boundary . . . . . . . . . . . 7

2.1.3 Gluing surfaces . . . . . . . . . . . . . . . . . . . . . . 9

2.2 Surfaces as combinatorial objects . . . . . . . . . . . 12

2.2 .1 Ribbon graphs . . . . . . . . . . . . . . 12

2.2.2 Classification of surfaces I: Existence . . . . . . . . . 18

2.3 The fundamental group of a surface . . . . . . . . . . . . 23

2.3.1 The fundamental group of a topological space . . . . . 23

2.3.2 Cayley graph and presentation complex . . . . . . . . 26

2.3.3 Classification of surfaces II: Uniqueness . . . . . . . . . . 28

2.4 Combinatorial versions of the fundamental group . . . . . . . . 29

2.4.1 A combinatorial description using a ribbon graph . . . . 29

2.4.2 A combinatorial description of the fundamental group using covers . . . . . . . . . . . . . 30

2.5 Cohomology of Surfaces . . . . . . . . . . . . . . 32

2.5.1 De Rham cohomology . . . . . . . . . . . . . . . 32

2.5.2 Ribbon graphs and cohomology . . . . . . . . . . . 37

2.5.3 The intersection form . . . . . . . . . . . . 41

2.6 Comments, references and further readings . . . . . . . . . 43

3 Vector bundles and connections $\quad 45$

3.1 Vector bundles . . . . . . . . . . . . . . . . . . 45

3.1.1 Definitions . . . . . . . . . . . . . . . . . 45

3.1.2 Constructions . . . . . . . . . . . . . . . 48

3.2 Vector bundles with structures . . . . . . . . . . . . . 49

3.2.1 Vector bundles over manifolds . . . . . . . . . . . . . 52

3.3 Connections . . . . . . . . . . . . . . . . . 53 
3.3.1 Koszul connection . . . . . . . . . . . . . . 54

3.3.2 Constructions . . . . . . . . . . . . . . . . . 55

3.3.3 Gauge equivalence and action of the gauge group . . . . 56

3.3.4 Holonomy along a path . . . . . . . . . . . . . 57

3.3.5 Holonomy and linear differential equations . . . . . . . . 58

3.3.6 Curvature . . . . . . . . . . . . . . . . . . 58

3.3.7 Flat connections . . . . . . . . . . . . . . . . 59

3.3.8 Connections preserving structures . . . . . . . . . 60

3.3.9 The holonomy map of a flat connection . . . . . . . . . 61

3.4 Combinatorial versions of connections . . . . . . . . . . . 62

3.4.1 Discrete connections on ribbon graphs . . . . . . . . . 62

3.4 .2 Local systems . . . . . . . . . . . . . . . 63

3.5 Four models of the representation variety . . . . . . . . . . 64

3.5.1 Flat connections and local systems . . . . . . . . . 65

3.5.2 Local systems and representations . . . . . . . . . . . . 66

3.5.3 Smooth and discrete connections . . . . . . . . . . 67

3.6 Comments, references and further readings . . . . . . . . 68

$\begin{array}{llr}4 & \text { Twisted Cohomology } & 69\end{array}$

4.1 De Rham version of twisted cohomology . . . . . . . . . . . . . 69

4.1.1 Motivation: variation of connection . . . . . . . . . . 69

4.1.2 De Rham version of the twisted cohomology . . . . . . . 72

4.2 A combinatorial version . . . . . . . . . . . . 76

4.2.1 The combinatorial complex . . . . . . . . . . . 77

4.2.2 The isomorphism Theorem . . . . . . . . . . . 78

4.2 .3 Duality . . . . . . . . . . . . . . . . . . . . 81

4.2.4 Proof of Theorem 4.1.7 . . . . . . . . . . . . . 84

4.3 Torsion . . . . . . . . . . . . . . . . . . . 84

4.3.1 Determinants . . . . . . . . . . . . . 85

4.3.2 An isomorphism between determinants . . . . . . . 85

4.3.3 The torsion of a metric complex . . . . . . . . . 87

4.3.4 The torsion of a flat metric connexion . . . . . . . . 87

4.3.5 Torsion and symplectic complexes . . . . . . . . . . . 89

4.3.6 Parallel metric on bundles . . . . . . . . . . . . . . . . 91

4.4 Comments, references and further readings . . . . . . . . . 93

$\begin{array}{llr}5 & \text { Moduli spaces } & \mathbf{9 4}\end{array}$

5.1 Moduli space and character variety . . . . . . . . . . . . . . 94

5.2 Topology and smooth structure . . . . . . . . . . . . . 9 95

5.3 Proof of the Theorem 5.2.6 . . . . . . . . . . . . . . 97

5.3.1 The Zariski closure of a group . . . . . . . . . . . . . 99 
5.3.2 More on the holonomy map . . . . . . . . . . . . . 102

5.3.3 The Zariski dense part is Zariski open and non-empty . . 104

5.3.4 The action is proper . . . . . . . . . . 106

5.3.5 The moduli space and its tangent space . . . . . . . . . 108

5.3.6 The complementary of the Zariski dense part . . . . . . . 109

5.4 Connected components . . . . . . . . . . . . . . . . 109

5.4.1 Connected components I: the compact case . . . . . . 109

5.4.2 Connected Components II : an invariant . . . . . . . . . 111

5.4.3 Connected components III: Milnor-Wood inequality . . . 113

5.4.4 Connected components IV: Goldman's work . . . . . . . 116

5.5 Comments, references and further readings . . . . . . . . 117

$\begin{array}{llr}6 & \text { Symplectic structure } & 118\end{array}$

6.1 Universal connections and smooth structures . . . . . . . . . 118

6.1 .1 Lifting Lemmas . . . . . . . . . . . . . . . . . . 118

6.1.2 Universal connections . . . . . . . . . . . . . . . . . . . 119

6.2 The symplectic form . . . . . . . . . . . . . . . . . . . 121

6.3 Classical mechanics and observables . . . . . . . . . . . . . . 122

6.4 Special observables I: Wilson loops and the Goldman algebra . . 123

6.5 Special observables II: Spin networks . . . . . . . . . . . . . 128

6.5.1 An observable on the space of all flat connections . . . . 129

6.5.2 Poisson bracket of spin network observables . . . . . . . 130

6.6 Volumes of moduli spaces . . . . . . . . . . . . . . . . 132

6.6.1 Witten's formula . . . . . . . . . . . . . . 132

6.6.2 Volume and the disintegrated measure . . . . . . . . 133

6.6.3 Characters and the disintegrated measure . . . . . . . 136

6.7 Comments, references and further readings . . . . . . . . . . 138

7 Three manifolds and integrality $\quad 140$

7.1 Integrality . . . . . . . . . . . . . . . . . . . 140

7.1.1 The Cartan form . . . . . . . . . . . . . . . . . 141

7.1.2 Integrating the Cartan form . . . . . . . . . . . . . . 142

7.2 Boundary of 3-Manifolds and Lagrangian submanifolds . . . . . 145

7.2.1 Submanifolds of the moduli space . . . . . . . . . . 146

7.2 .2 Isotropic submanifold . . . . . . . . . . . . . . . . 147

7.2.3 Chern-Simons invariants . . . . . . . . . . . . . 147

7.2.4 Chern Simons action difference and the symplectic form 150

7.3 Comments, references and further readings . . . . . . . . . . . 153

Index 


\section{Chapter 2}

\section{Surfaces}

In this introductory chapter, we give two definitions of surfaces. First as a differential geometric object, then as a combinatorial object: a ribbon graph. We use this description first to give the classification of oriented surfaces then to describe the fundamental objects we are going to deal with in this book: the fundamental group and the cohomology in its various guises. The style of this chapter is that of a crash course mainly meant to settle ideas and notation for the next chapters.

The main point of this introductory chapter is to go back and forth between the two languages that we are going to use in the first chapters of this monograph: differential geometry and combinatorics.

\subsection{Surfaces as 2-dimensional manifolds}

\subsubsection{Basic definitions}

In this section, we will recall the point of view of differential geometers on surfaces.

Definition 2.1.1 A surface is a connected two-dimensional smooth manifold.

We assume that the reader is familiar with the definition of a manifold. Nevertheless, in order to fix ideas and notation, let us briefly discuss the definition above. We assume first that $S$ is a metrisable topological space, in particular Hausdorff. A two-dimensional chart for such a topological space $S$ is a pair $(U, \varphi)$ where $U \subset S$ is an open subset and $\varphi$ is a map from $U$ to $\mathbb{R}^{2}$, where $V=\varphi(U)$ is open in $\mathbb{R}^{2}$ and $\varphi$ is a homeomorphism onto its image. The map $\varphi$ is called the coordinate of the chart. A collection of charts $\left\{\left(U_{i}, \varphi_{i}\right) \mid i \in I\right\}$ 
is called an atlas for $S$ if $S=\bigcup U_{i}$. An atlas is called smooth or $C^{\infty}$ (we use these two expressions synonymously) if the coordinate changes

$$
\varphi_{i} \circ \varphi_{j}^{-1}: \varphi_{j}\left(U_{i} \cap U_{j}\right) \rightarrow \varphi_{i}\left(U_{i} \cap U_{j}\right)
$$

are smooth functions for all $i, j \in I$. The reason to introduce a smooth atlas is the desire to be able to talk about smooth functions on $S$. If smooth functions exists, we definitely want the coordinates $\varphi_{i}$ and there inverses $\varphi_{i}^{-1}$ to be smooth.

So let us just pretend that they are smooth.

If the notion of smooth functions is any good, then given a smooth function $\varphi: S \rightarrow \mathbb{R}$ also the composition or the function seen in the chart

$$
\varphi_{i}^{-1} \circ \varphi: \varphi_{i}\left(U_{i}\right) \rightarrow \mathbb{R}
$$

should be smooth. But these are just functions from subsets of $\mathbb{R}^{2}$ to $\mathbb{R}$ and for such functions we know what smoothness means. Thus we can just define a function $\varphi$ to be smooth if the function seen in all charts is smooth. In fact, it suffices to check this for only one particular chart around each point as we were clever enough to demand the coordinate changes to be smooth themselves. Thus we may talk about smooth functions on a surface like we may talk about smooth functions on $\mathbb{R}^{2}$.

Obvious examples of surfaces are given by connected open subsets of $\mathbb{R}^{2}$. Indeed, if $U \subset \mathbb{R}^{2}$ and $i: U \rightarrow \mathbb{R}^{2}$ is the inclusion map then $\{(U, i)\}$ is a smooth atlas for $U$. Geometric intuition tells us that all these examples are oriented, while the Möbius strip (Figure 2.1) is not. One way to formalise this is via the derivatives of the coordinate changes:

Definition 2.1.2 Let $S$ be a surface with atlas $\left\{\left(U_{i}, \varphi_{i}\right) \mid i \in I\right\}$. S is called oriented if the Jacobians

$$
\operatorname{Jac}\left(\varphi_{i}, \varphi_{j}\right):=\operatorname{det}\left(\mathrm{D}\left(\varphi_{i} \circ \varphi_{j}^{-1}\right)\right)
$$

are positive for any $i, j \in I$.

\subsubsection{Surfaces with boundary}

In order to study surfaces, we shall often cut a surface into pieces along a curve or to glue two such pieces in order to obtain a new surface. The pieces occurring in such geometric operations are not surfaces in the sense of Definition 2.1.1. 


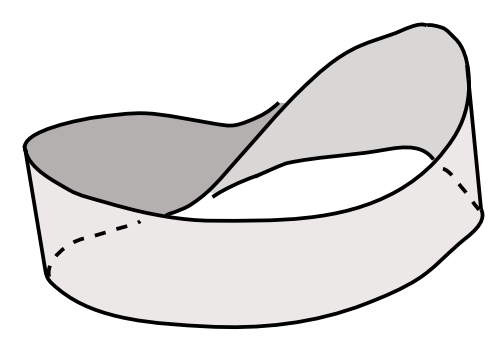

Figure 2.1: The Möbius strip

Thus, we need a more general definition in order to deal with these pieces as well. For this, we need another model space. Let

$$
H^{+}:=\left\{(x, y) \in \mathbb{R}^{2} \mid y \geq 0\right\}
$$

be the closed upper half plane. The upper half plane has a boundary

$$
\partial H^{+}:=\left\{(x, y) \in \mathbb{R}^{2} \mid y=0\right\}
$$

when considered as a subset of $\mathbb{R}^{2}$. Given a metrisable space $S$, a twodimensional chart with boundary is a pair $(U, \varphi)$ where $U$ is an open subset of $S$ and $\varphi: U \rightarrow V$ is a homeomorphism onto an open subset $V \subset H^{+}$, which is again called the coordinate of the chart. The subset

$$
\partial U:=\varphi^{-1}\left(\varphi(U) \cap \partial H^{+}\right) \subset U
$$

will be called the boundary of U. In order to define a smooth atlas of charts with boundary, we need to define a notion of smoothness for functions between open subsets of $H^{+}$.

Definition 2.1.3 Let $V_{1}, V_{2}$ be open subsets of $H^{+}$. A function $f: V_{1} \rightarrow V_{2}$ is called smooth if there exists an open subset $\widetilde{V}_{1}$ of $\mathbb{R}^{2}$ with $\widetilde{V}_{1} \cap H^{+}=V_{1}$. and a smooth function $\widetilde{f}: \widetilde{V}_{1} \rightarrow \mathbb{R}^{2}$ such that $f=\left.\widetilde{f}\right|_{V_{1}}$.

An atlas of charts with boundary $\left\{\left(U_{i}, \varphi_{i}\right) \mid i \in I\right\}$ is smooth if the coordinate changes

$$
\varphi_{i} \circ \varphi_{j}^{-1}: \varphi_{j}\left(U_{i} \cap U_{j}\right) \rightarrow \varphi_{i}\left(U_{i} \cap U_{j}\right)
$$

are smooth in the sense of Definition 2.1.3. Then we may define: 
Definition 2.1.4 $A$ surface with boundary is a metrisable space $S$ together with a smooth atlas of charts with boundary.

We define in a similar fashion as before the notion of smooth functions on a manifold with boundary. In order to define the boundary of a surface with boundary one needs to proof the following lemma:

Lemma 2.1.5 Let $S$ be a surface with boundary and let $\left(U_{1}, \varphi_{1}\right),\left(U_{2}, \varphi_{2}\right)$ be charts with boundary of $S$. Suppose $x \in \partial U_{1} \cap U_{2}$, in other words $x$ is a boundary point of $U_{1}$. Then $x \in \partial U_{2}$ is a boundary point of $U_{2}$.

Exercise 2.1.6 Deduce Lemma 2.1.5 from the implicit function Theorem.

In fact boundary points are not only preserved by smooth maps but even by continuous maps. To see this, however, requires some algebraic topology. By Lemma 2.1.5 the notion of being a boundary point of a chart does not depend on the particular chart. Thus we may define:

Definition 2.1.7 Let $S$ be a surface with boundary. A point $x \in S$ is called $a$ boundary point of $S$ if $x \in \partial U$ for some (and hence any) chart $(U, \varphi)$ containing it. The set of boundary points of $S$ is denoted $\partial S$.

In order to glue surfaces along their boundaries we need to know how they look like in a neighbourhood of a boundary component. This is described by the following lemma:

Lemma 2.1.8 [COllar Lemma] Let $M$ be a surface with boundary $\partial M$ and $c \subset \partial M$ a connected component. Then there exists a neighbourhood $U$ of $c$ in $M$ and a diffeomorphism $\psi: U \rightarrow V$ onto a subset $V \subset \mathbb{R}^{2}$ of the form $V \cong c \times[0,1)$ mapping $c$ onto $c \times\{0\}$.

The neighbourhood $U$ will be called a collar neighbourhood or tubular neighbourhood of $c$. The proof of Lemma 2.1.8 is easy if one uses Riemannian geometry and in particular exponential charts. There is no short elementary proof though and we omit the proof.

\subsubsection{Gluing surfaces}

Let $S_{1}, S_{2}$ be two surface with boundary. Let $c_{1}$ and $c_{2}$ be two diffeomorphic components of $\partial S_{1}$ and $c_{2}$ of $\partial S_{2}$. Intuitively, the gluing of the two surfaces along these boundary components is depicted by the Picture 2.3. We sketch 


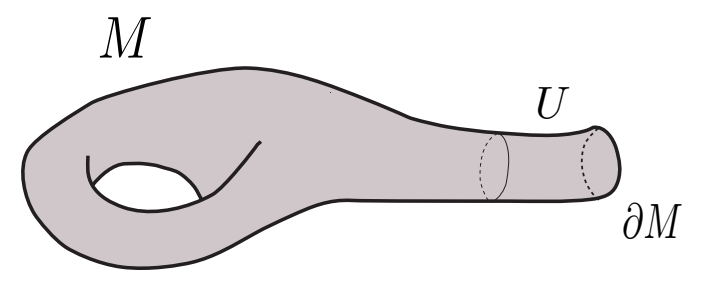

Figure 2.2: A collar neighbourhood

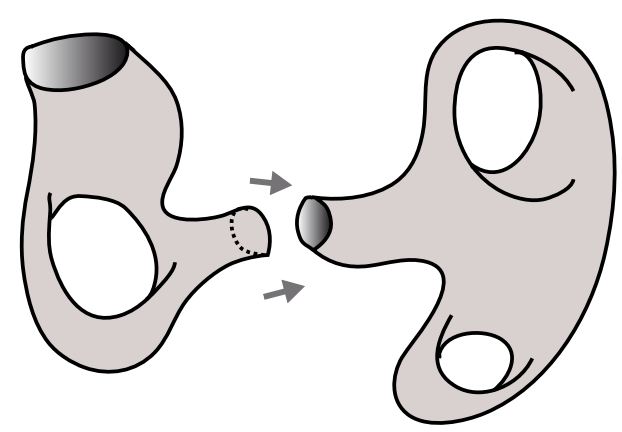

Figure 2.3: Gluing two surfaces

the formalisation of this gluing procedure now. Let notation be as above and let $\varphi: c_{1} \rightarrow c_{2}$ be a diffeomorphism. We define a topological space

$$
S_{1} \cup_{\varphi} S_{2}
$$

called the gluing of $S_{1}$ and $S_{2}$ along $\varphi$ as follows: on the disjoint union $S_{1} \amalg S_{2}$ we consider the equivalence relation generated by

$$
x \sim y \Leftrightarrow y=\varphi(x)
$$

for $x \in c_{1}, y \in c_{2}$. Then $S_{1} \cup_{\varphi} S_{2}$ is the quotient of $S_{1} \coprod S_{2}$ by this equivalence relation.

Exercise 2.1.9 Show that the space $S_{1} \cup_{\varphi} S_{2}$ is metrisable. 
An atlas for $S_{1} \cup_{\varphi} S_{2}$ is now constructed as the union of three subsets: Firstly, we take a smooth atlas $\left\{\left(U_{i}, \varphi_{i}\right) \mid i \in I\right\}$ for $S_{1} \backslash c_{1}$ and a smooth atlas $\left\{\left(U_{j}, \varphi_{j}\right) \mid j \in J\right\}$ for $S_{2} \backslash c_{2}$. Denote by $i_{1}: S_{1} \hookrightarrow S_{1} \cup_{\varphi} S_{2}, i_{2}: S_{2} \hookrightarrow S_{1} \cup_{\varphi} S_{2}$ the canonical inclusions. Then

$$
\left\{\left(i_{1}\left(U_{i}\right), \varphi_{i} \circ i_{1}^{-1}\right) \mid i \in I\right\} \cup\left\{\left(i_{2}\left(U_{j}\right), \varphi_{j} \circ i_{2}^{-1}\right) \mid j \in J\right\}
$$

is an atlas for the complement of the gluing curve in $S_{1} \cup_{\varphi} S_{2}$. It remains to construct a chart around this gluing curve which is compatible with the charts above. For this we use Lemma 2.1.8: Let $U_{1}, U_{2}$ be collar neighbourhoods of $c_{1}, c_{2}, \psi_{1}$ a diffeomorphism from $U_{1}$ onto $c_{1} \times(-1,0]$ (mapping $c_{1}$ to $\left.c_{1} \times\{0\}\right)$ and $\psi_{2}$ a diffeomorphism from $U_{2}$ onto $c_{2} \times[0,1)$ (mapping $c_{2}$ to $c_{2} \times\{0\}$ ). We consider the open subset $O:=U_{1} \cup_{\varphi} U_{2}$ of $S_{1} \cup_{\varphi} S_{2}$ and fix an embedding $\iota: c_{2} \times(-1,1) \rightarrow \mathbb{R}^{2}$. This is always possible as $c_{2}$ is either an interval or a circle. We define coordinates for $O$ by

$$
\psi: O \rightarrow \mathbb{R}^{2}, \quad x \mapsto\left\{\begin{array}{lll}
\iota \circ(\varphi, i d) \circ \psi_{1}(x) & \text { if } & x \in U_{1} \\
\iota \circ \psi_{2}(x) & \text { if } & x \in U_{2}
\end{array}\right.
$$

Then one has:

Proposition 2.1.10 $S_{1} \cup_{\varphi} S_{2}$ is a surface with boundary with smooth atlas given by

$$
\left\{\left(i_{1}\left(U_{i}\right), \varphi_{i} \circ i_{1}^{-1}\right) \mid i \in I\right\} \cup\left\{\left(i_{2}\left(U_{j}\right), \varphi_{j} \circ i_{2}^{-1}\right) \mid j \in J\right\} \cup\{O, \psi\} .
$$

Exercise 2.1.11 Prove Proposition 2.1.10.

Exercise 2.1.12 Let $S_{1}, S_{2}$ be oriented surfaces with boundary and $c_{1}, c_{2}$ be connected components of the respective boundaries. Then $c_{1}, c_{2}$ carry an induced orientation. Suppose now that $\varphi: c_{1} \rightarrow c_{2}$ is an orientation reversing diffeomorphism. Then there is a unique orientation of $S_{1} \cup_{\varphi} S_{2}$ compatible with the orientations of $S_{1}$ and $S_{2}$

So far the only examples of surfaces we have seen are open subsets of $\mathbb{R}^{2}$. Proposition 2.1.10 allows us to construct many other examples of surfaces. For example the closed unit disc in $\mathbb{R}^{2}$ is a surface with boundary and gluing two copies of this along the unit circle gives rise to the two-sphere $S^{2}$. In order to study such gluing more systematically we shall introduce some combinatorial notions. 


\section{$2.2 \quad$ Surfaces as combinatorial objects}

\subsubsection{Ribbon graphs}

A graph is a collection of points (vertices) which are joined by some lines (edges) as in Picture 2.4. If furthermore we choose an orientation on the edges, we say the graph is oriented. The following definition provides a formal description of the objects in the picture:

Definition 2.2.1 An oriented graph $\Gamma$ is a triple $\Gamma=(V, E, \varphi)$, where $V$ and $E$ are sets whose elements are called vertices and edges respectively and

$$
\varphi: E \rightarrow V \times V, \quad e \mapsto\left(e_{-}, e_{+}\right)
$$

is a map. The vertex $e_{-}$is called the origin of e while the vertex $e_{+}$is called the terminus of $e$.

$A$ graph is a pair $(\Gamma, I)$, where $\Gamma=(V, E, \varphi)$ is an oriented graph and $I: E \rightarrow E, e \mapsto \bar{e}$ is a fixed point free involution on $E$ satisfying

$$
\bar{e}_{+}=e_{-}, \quad \bar{e}_{-}=e_{+} .
$$

A pair $(e, \bar{e})$ in $E^{2}$ is called a geometric edge of $(\Gamma, I)$.

The geometric realisation $|\Gamma|_{I}$ of a graph $(\Gamma, I)$ is the topological space

$$
|\Gamma|_{I}=E \times[0,1] / \sim
$$

where $\sim$ is the equivalence relation generated by the following relations:

- $(e, t) \sim(\bar{e}, 1-t)$.

- If $e, f \in E$ with $e_{-}=f_{-}$then $(e, 0) \sim(f, 0)$.

- If $e, f \in E$ with $e_{+}=f_{+}$then $(e, 1) \sim(f, 1)$.

The geometric realisation of a finite graph cannot always be drawn on a plane with non intersecting edges. However, one can draw a graph in the plane, as if it were the projection of a graph in space, so that edges appears to cross over or under as in Figure 2.4.

We will be interested with graphs with some extra structure. For this we need the notion of a cyclic ordering on a finite set $S$. By this we shall mean a bijection $s: S \rightarrow S$ such that for any $x \in S$ the orbit $\left\{s^{n}(x)\right\}$ is $S$. Then given $x \in S$ we will call $s(x)$ the (cyclic) successor of $x$ and $s^{-1}(x)$ the (cyclic) predecessor of $x$. 


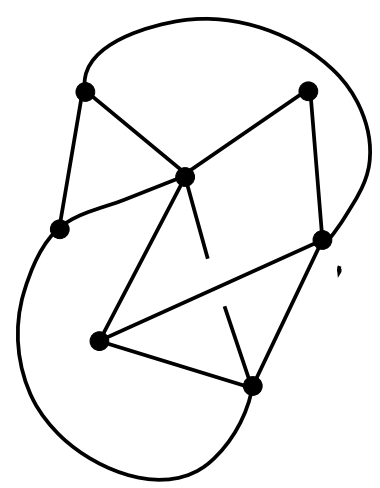

Figure 2.4: A graph

Definition 2.2.2 [RIBbon GRAPH] Let $(\Gamma, I)$ be a graph. For $v \in V$, the star of $v$ is

$$
E_{v}:=\left\{e \in E \mid e_{-}=v\right\},
$$

the set of edges starting from $v$. A ribbon graph is the data given by a graph and a cyclic ordering on the star of every vertex.

Any planar graph is a ribbon graph. More precisely, given an embedding of a graph ${ }^{1}$ into $\mathbb{R}^{2}$ the orientation of $\mathbb{R}^{2}$ induces a cyclic ordering on each of the sets $E_{v}$. (We may take a small circle around each vertex intersecting each edge only once. Then the circle obtains an orientation from $\mathbb{R}^{2}$ and this defines the ordering.

One can always represent a ribbon graph on the plane as the projection of a graph in space - so again with edges that may cross, over or under - so that the cyclic ordering of the edges coincides with the cyclic ordering arising from the orientation in the plane as in Figure 2.5.)

Exercise 2.2.3 Show that any embedding of a graph into an oriented surface gives rise to a cyclic orientation on the sets $E_{v}$.

We will now go the other way around and construct surfaces starting from ribbon graphs. As a first step we will embed a ribbon graph into an open (in other words non-compact) oriented surface, even though we remarked a that it is not always possible to do so in the plane.

\footnotetext{
${ }^{1}$ When we talk about an embedding of an graph into a topological space we actually mean an embedding of the geometric realisation of that graph. This slight abuse of language will be used throughout.
} 


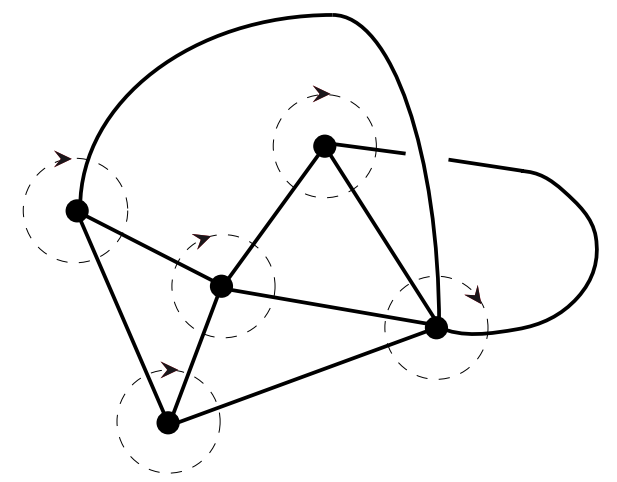

Figure 2.5: Cyclic ordering of edges in a planar graph

Lemma 2.2.4 Every ribbon graph can be embedded into an oriented surface such that its cyclic orderings are induced from the orientation of the surface.

Proof: We construct a surface $S$ by gluing certain elementary pieces: for every vertex $v \in V$ define a subset $U_{v} \subset \mathbb{R}^{2}$ with $\left|E_{v}\right|$ boundary components labelled by the elements of $E_{v}$ of the form depicted in Figure 2.6 (Only the straight lines of the boundary are part of the surface.) We do the labelling in the following way: We start with an arbitrary edge $e \in E_{v}$. Then the next boundary component in the counterclockwise sense is labelled by $s_{v}(e)$ and so on.

Similarly we attach to each edge $e$ a strip $V_{e}$ as depicted in Figure 2.7 whose two boundary components (again only the straight lines are part of the surface) are labelled by $e_{+}$and $e_{-}$. Now for each $e \in E$ we glue the connected component of $\partial V_{e}$ labelled by $v$ with the connected component of $\partial U_{v}$ labelled by $e$ (if $v$ is the origin of $e$ ) or $\bar{e}$ (if $v$ is the terminus). If we do this in an orientation reversing way then by Exercise 2.1.12 we obtain an oriented surface. At each vertex $v$ the orientation on the corresponding star is preserved under the gluing process. Thus, by construction the orientation is compatible with $s_{v}$. Q.E.D.

The open surface constructed from a ribbon graph as in the proof of Lemma 2.2.4 is called the associated ribbon surface of that graph. In order to embed ribbon graphs into closed surfaces one has to close the holes in the ribbon surface. For a graph as depicted in Figure 2.8 this is easy: we should glue discs inside the holes so that we obtain a sphere. Observe that the boundaries of these faces can be read off from the picture as follows: start with any edge 


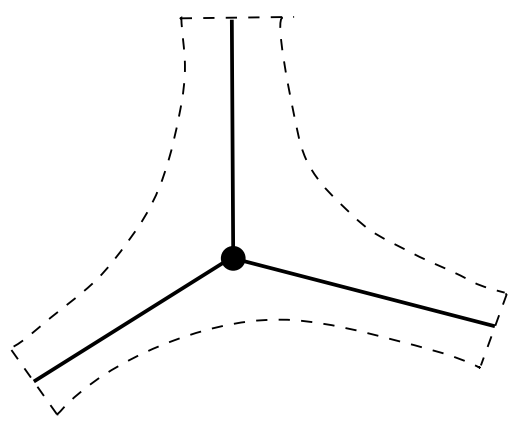

Figure 2.6: A surface associated with a vertex

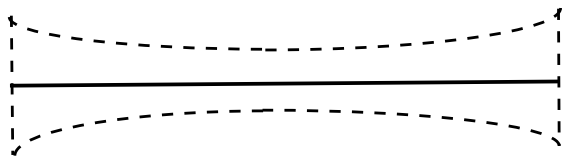

Figure 2.7: A surface associated with an edge

$e$ and follow this edge to the next vertex. Then take the successor of $\bar{e}$ and continue until you get a closed curve. The curve constructed this way will be the boundary of a disc in the surface we want to construct.

This procedure works also in more complicated situations as depicted in Figure 2.9 and we formalise it in the following definition:

Definition 2.2.5 Let $\Gamma$ be a ribbon graph. A face is an equivalence class - up to cyclic permutation - of n-tuples $\left(e_{1}, \ldots, e_{n}\right)$ of edges such that $e_{p}^{+}=e_{p+1}^{-}$ and $s_{e_{p}^{+}}\left(\bar{e}_{p}\right)=e_{p+1}$ for all $1 \leq p \leq n$, where the addition is modulo $n$.

Note that every oriented edge is contained in a unique face.

Definition 2.2.6 A graph $\Gamma$ embedded in a surface $S$ is filling if each connected component of $S \backslash|\Gamma|$ is diffeomorphic to the disc. 


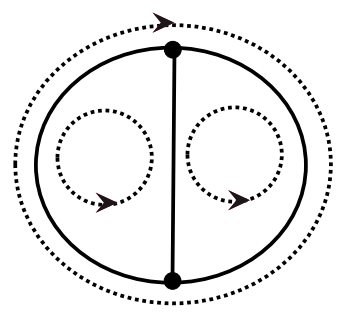

Figure 2.8: A graph with three faces

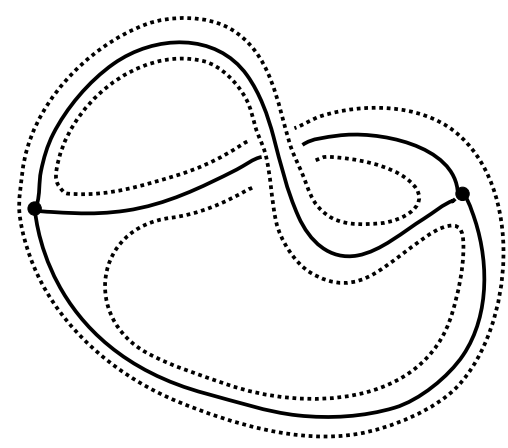

Figure 2.9: A graph with one face 
Then we have:

Proposition 2.2.7 Every ribbon graph $\Gamma$ has a filling embedding into a compact oriented surface $S$. The connected components of $S \backslash|\Gamma|$ are in bijection with the faces of $\Gamma$.

Proof: The edges of a face define a circle in the ribbon surface. We glue a disc for each of these circles. We thus obtain a closed surface. Q.E.D.

Exercise 2.2.8 What surfaces do you obtain by performing the above construction from the ribbon graphs depicted by Figures 2.8 and 2.9 respectively?

The surface obtained from a ribbon graph by Proposition 2.2.7 is in fact unique in a very strong sense. To make this precise, we will need the following basic fact from point-set topology:

Lemma 2.2.9 [Clutching Lemma] Let $X=U \cup V$ be a decomposition of a topological space $X$ into closed subsets $U, V$. If $f_{1}: U \rightarrow Y$ and $f_{2}: V \rightarrow Y$ are continuous maps from $U$ and $V$ into some topological space $Y$ with $\left.f_{1}\right|_{U \cap V}=$ $\left.f_{2}\right|_{U \cap V}$ then the induced map $f: X \rightarrow Y$ is continuous.

Using this we can show the following weak uniqueness result:

Proposition 2.2.10 Let $\Gamma \subset S, \Gamma^{\prime} \subset S^{\prime}$ be filling ribbon graphs of compact oriented surfaces and let $\varphi: \Gamma \rightarrow \Gamma^{\prime}$ be an isomorphism of ribbon graphs. Then $\varphi$ induces an orientation-preserving homeomorphism $\varphi:|\Gamma| \rightarrow\left|\Gamma^{\prime}\right|$ of geometric realisations and this extends to a homeomorphism $S \rightarrow S^{\prime}$.

Proof: It is easy to see that $\varphi$ extends to the geometric realisation and further to the closure of the associated ribbon surfaces. Let

$$
S \backslash|\Gamma|=\coprod_{f \in F} D_{f}, \quad S^{\prime} \backslash\left|\Gamma^{\prime}\right|=\coprod_{f \in F} D_{f}^{\prime} .
$$

Let $S_{\Gamma}$ and $S_{\Gamma^{\prime}}$ be the ribbon surfaces associated with $|\Gamma|,\left|\Gamma^{\prime}\right|$. Then

$$
S=\overline{S_{\Gamma}} \cup\left(\coprod_{f \in F} \overline{d_{f}}\right),
$$

where $d_{f}$ is a disc slightly smaller than $D_{f}$ and

$$
\overline{S_{\Gamma}} \cap\left(\coprod_{f \in F} \overline{d_{f}}\right)=\coprod \partial \overline{d_{f}}
$$


is a union of circles. The same statement is true for $S^{\prime}$. By Lemma 2.2.9 it then suffices to construct for each $f \in F$ a homeomorphism $\overline{d_{f}} \rightarrow \overline{d_{f}^{\prime}}$ which agrees on the boundary $\partial \overline{d_{f}}$ with the extension of $\varphi$ to $\overline{S_{\Gamma}}$. But if $\psi: \mathbb{T}^{1} \rightarrow \mathbb{T}^{1}$ is any given homeomorphism of circles then there is an obvious way to extend it to the corresponding discs. Indeed, every element $x$ of the disc $D$ may be written in polar coordinates as

$$
x=r e^{i \theta}
$$

for some $r \in[0,1]$ and some $e^{i \theta} \in \mathbb{T}^{1}$. But then we may simply define $\psi\left(r e^{i \theta}\right):=$ $r \varphi\left(e^{i \theta}\right)$ to obtain the desired homeomorphism. Q.E.D.

Combining this with Proposition 2.2.7 we obtain:

Corollary 2.2.11 For any ribbon graph $\Gamma$ there exists a unique compact oriented surface $S_{\Gamma}$ (up to homeomorphism) such that $\Gamma$ can be embedded as a filling ribbon graph into $S_{\Gamma}$.

Corollary 2.2.11 will enable us to classify surfaces up to homeomorphism. A classification up to diffeomorphism is possible along the same lines, but it would require a stronger form of the corollary. Namely, one would have to prove that any homeomorphism of surfaces is homotopic to a diffeomorphism and thus a ribbon graph determines the surface even up to diffeomorphism. This becomes harder to prove and we will not pursue a classification up to diffeomorphisms here.

Corollary 2.2.11 allows us to construct surfaces starting from ribbon graphs. The following proposition is a converse to this corollary.

Proposition 2.2.12 Every compact oriented surface admits a filling ribbon graph.

$\mathrm{V}$

We will not prove Proposition 2.2.12 here. The problem of constructing a filling ribbon graph is equivalent to the probably more familiar problem of constructing a triangulation of a given surface. Indeed the set of edges of the triangulation will yield a filling ribbon graph. There are several standard methods in order to produce a triangulation (and hence a filling ribbon graph), but any of them uses some basic results from either Riemannian geometry or Morse theory, which we do not want to discuss here.

\subsubsection{Classification of surfaces I: Existence}

By Corollary 2.2.11 a convenient description of compact surfaces is given by their ribbon graphs. We consider a family $\Gamma_{g}, g \geq 1$ of ribbon graphs given 
as follows: Take $g$ copies of the graph shown in Figure 2.10 and glue them together as shown in Figure 2.11 to produce the graph with g petals.

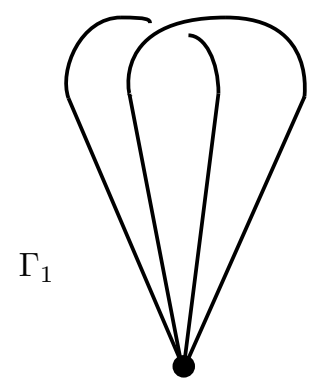

Figure 2.10: The graph $\Gamma_{1}$

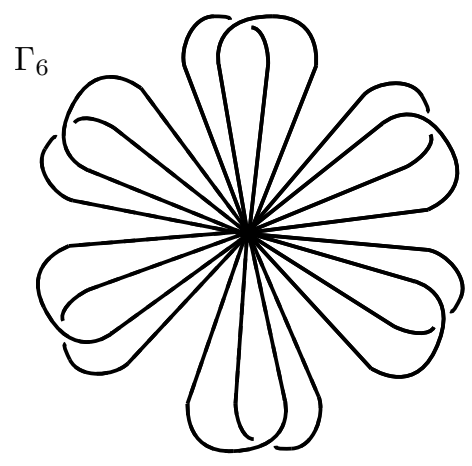

Figure 2.11: The graph $\Gamma_{g}$ for $g=6$

The graph $\Gamma_{1}$ has one face and with some imagination one sees that the associated surface $S_{1}:=S_{\Gamma_{1}}$ is a torus. Similarly, each copy of $\Gamma_{1}$ in $\Gamma_{g}$ gives rise to a torus with a hole and the holes of two consecutive tori are glued together. Thus, after some gymnastics, $S_{g}:=S_{\Gamma_{g}}$ is the surface of a handle body with $g$ handles.

There is another description of $S_{g}$ which turns out to be useful: Let $D_{g}$ be the disc in $S_{g}$ corresponding to the unique face of $\Gamma_{g}$. Then $S_{g}$ is obtained from gluing the boundary of $D_{g}$. Each (oriented) edge of $\Gamma_{g}$ occurs precisely once in the boundary of $D_{g}$. More precisely, let $a_{i}, b_{i}$ be the two edges of the 


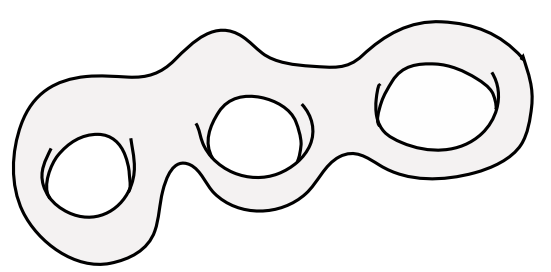

Figure 2.12: The surface $S_{3}$

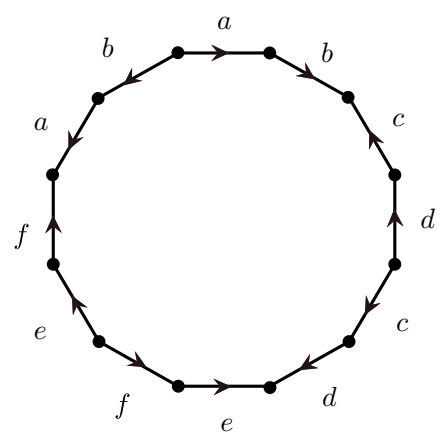

Figure 2.13: Constructing $S_{3}$ by gluing a disc

$i$-th copy of $\Gamma_{1}$ in $\Gamma_{g}$. Then the boundary of $D_{g}$ is given by the series of edges

$$
a_{1}, b_{1}, a_{1}^{-1}, b_{1}^{-1}, \ldots, a_{g}, b_{g}, a_{g}^{-1}, b_{g}^{-1} .
$$

For $g=3$, this is depicted in Figure 2.13.

It is convenient to define $S_{0}:=S^{2}$, the two-sphere. Then we can state one-half of the classification of surfaces as follows:

Theorem 2.2.13 Every compact oriented surface $S$ is homeomorphic to one of the surfaces $S_{g}$ for $g \geq 0$.

Proof: Using Proposition 2.2.12, we choose a filling ribbon graph $\Gamma$ for $S$. If the graph has no edges, then $S$ must be the two-sphere $S^{2}$. Thus we may assume that $\Gamma$ has at least one edge. We will now deform the graph $\Gamma$ into a graph $\Gamma_{g}$ without changing its filling property. The theorem will then follow from Corollary 2.2.11. Suppose firstly, that $\Gamma$ has more than one face. Then 


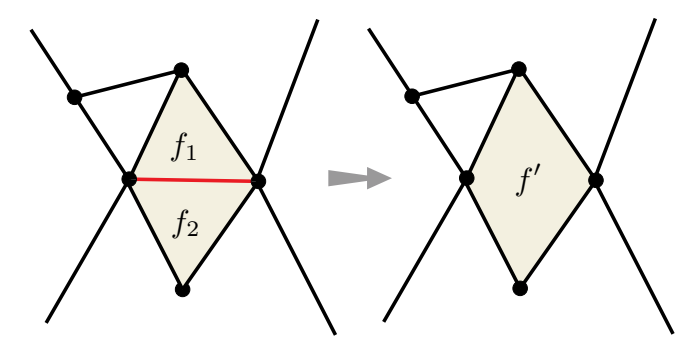

Figure 2.14: Reducing the number of faces

there exists a geometric edge $(e, \bar{e})$ such that $e$ and $\bar{e}$ are contained in different faces. Let $\Gamma^{\prime}$ be the graph obtained from $\Gamma$ by deleting $e$ and $\bar{e}$. Then $\Gamma^{\prime}$ is still filling for $S$ but has one face less as in Figure 2.14. Iterating this process we end up with a filling graph with only one face. Thus we may assume that $\Gamma$ has only one face.

Secondly, we reduce the number of vertices as in Figure 2.15. Let $\Gamma=(V, E)$ be a graph embedded in $S$ by the map $\varphi$. If $\Gamma$ have more than two vertices, there exists an edge $e$ joining the two different vertices $e_{+}$and $e_{-}$. Let $\Gamma^{\prime}=$ $\left(V^{\prime}, E^{\prime}, \varphi^{\prime}\right)$ where

- the new set of vertices is obtained by crushing the two vertices $e_{+}$and $e_{-}$to one new vertex $e_{0}, V^{\prime}=\left(V \backslash\left\{e_{+}, e_{-}\right\}\right) \cup\left\{e_{0}\right\}$,

- the new set of edges is $E^{\prime}=E \backslash\{e, \bar{e}\}$

- we define the new embedding $\varphi^{\prime}$ by sending the new vertex $e_{0}$ to a point in the geometric image of $\varphi(e)$ and extending it to the edges which previously started from $e_{ \pm}$. Such a construction only requires to work in a neighbourhood of $e$ and can be given a precise model.

Again this reduction of vertices does not change the fact that $\Gamma$ is filling and reduces the number of vertices by one without increasing the number of faces. Hence we may assume that $\Gamma$ has only one vertex and one face. This means that $S$ is obtained by gluing the boundary of a polygon. The sides of the polygon are labelled by the edges of $\Gamma$ and by definition of face every edge occurs precisely one. The gluing finally given by identifying $e$ and $\bar{e}$ with reversed orientation.

If there are no edges left, then $S$ is the two-sphere $S^{2}$ and we are done. Thus assume there are still edges left. We will call a pair of geometric edges 


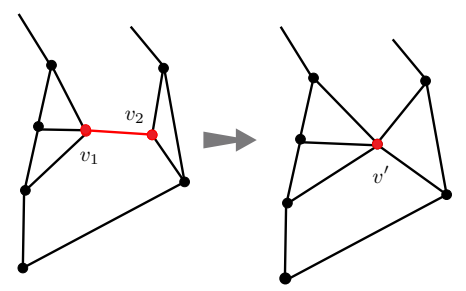

Figure 2.15: Reducing the number of vertices

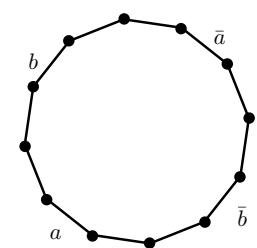

Figure 2.16: Linked Edges

$((a, \bar{a}),(b, \bar{b}))$ linked if their relative position is as in Figure 2.16. We claim that any geometric edge is linked to at least one other geometric edge. Indeed, from the ribbon graph point of view, a geometric edge which is not linked to any other edge would produce an additional face contradicting our assumption that there is only one face. Thus we are left to prove the following claim:

Given a linked pair $(a, \bar{a}),(b, \bar{b})$ of geometric edges, there is a way to rearrange the labelling of the polygon without changing the resulting quotient space such that $a, b, \bar{a}, \bar{b}$ appears as a subsequence and no subsequence of this type is destroyed during this process.

Indeed, if the claim is true then we can relabel the edges of the polygon in such a way that we obtain one of the graphs $\Gamma_{g}$.

For the proof of the claim we give an explicit construction which consists of adding and deleting certain edges of the graph. Such a procedure is depicted in Figure 2.17. The figure has to be read as follows. The main horizontal step is as follows, we add an edge which is dotted in the picture. We therefore obtain two faces have different colours in the picture. Then we erase in the graph the green edge, which has the effect on the polygon to glue together these two green lines in the red one. Then we repeat this procedure two times as is depicted in the picture. We observe that not consecutive subsequence of the form $c, d, \bar{c}, \bar{d}$ have been destroyed in this construction. The resulting 
surface (which is homeomorphic to the one before) is thus brought into a new position such that all edges lie on the boundary. In the final picture we have created an additional subsequence of the form $a, b, \bar{a}, \bar{b}$ which proves the claim and finishes the proof of the theorem. Q.E.D.

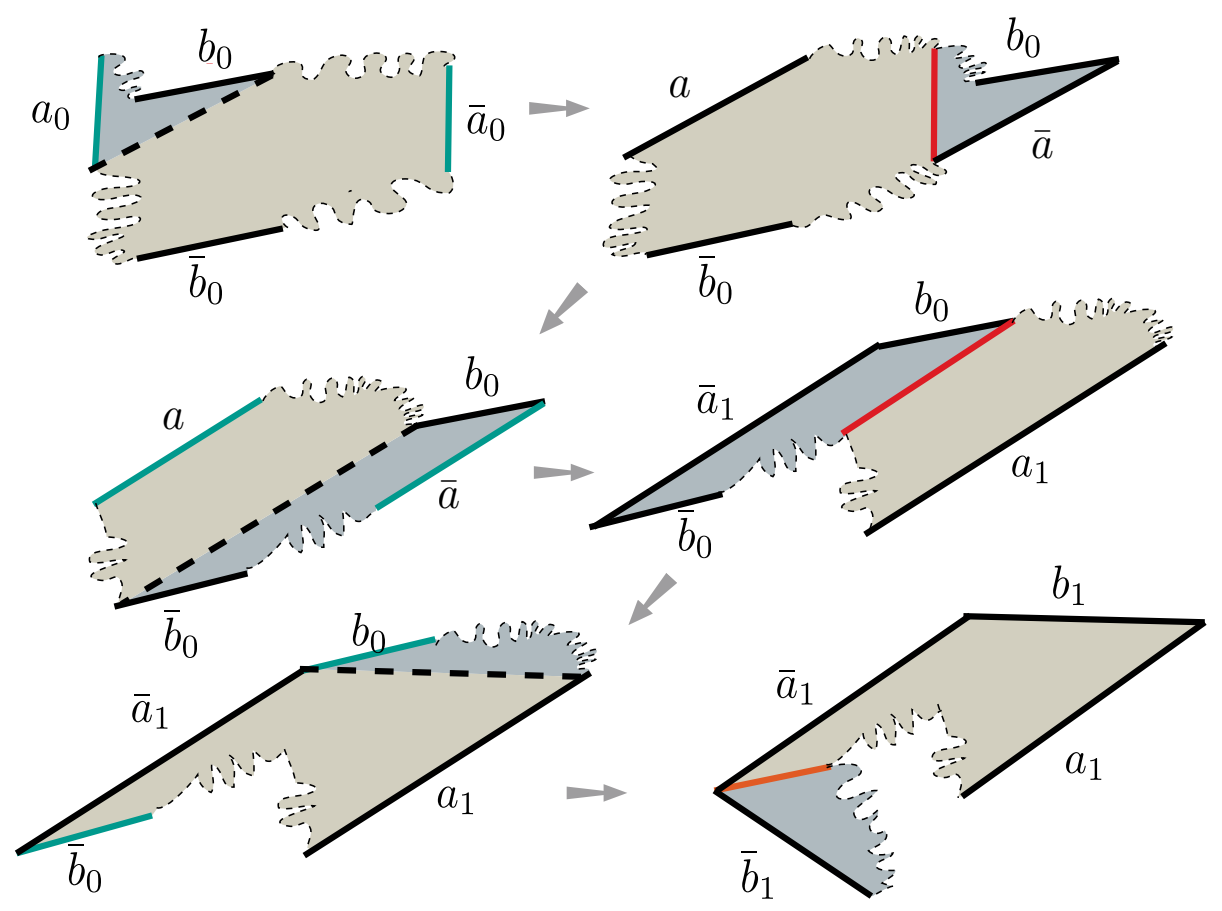

Figure 2.17: Moving linked edges into generic position

\subsection{The fundamental group of a surface}

\subsubsection{The fundamental group of a topological space}

The result obtained in Theorem 2.2.13 is not yet a complete classification of compact oriented surfaces as we do not know yet whether some of the surfaces $S_{g}$ for distinct $g$ are homeomorphic. In order to show that this does not happens, we need an invariant that distinguishes the surfaces $S_{g}$ from each other.

This invariant is the fundamental group- the central object of these series of lectures - and we briefly recall its definition:

Definition 2.3.1 Let $X, Y$ be a topological spaces. 
(i) A (parametrised) loop in $X$ based at $x_{0} \in X$ is a continuous map $\gamma$ : $[0,1] \rightarrow X$ with $\gamma(0)=\gamma(1)=x_{0}$. Denote by $\Omega\left(X, x_{0}\right)$ the set of loops in $X$ based at $x_{0}$.

(ii) The composition of two based loops $\gamma_{0}, \gamma_{1} \in \Omega\left(X, x_{0}\right)$ is defined to be

$$
\left(\gamma_{0} * \gamma_{1}\right)(t):= \begin{cases}\gamma_{0}(2 t), & 0 \leq t \leq \frac{1}{2} \\ \gamma_{1}(2 t-1), & \frac{1}{2} \leq t \leq 1\end{cases}
$$

(This is continuous by Lemma 2.2.9.)

(iii) Let $f_{0}, f_{1}: Y \rightarrow X$ be continuous maps which agree on a subset $A \subset Y$. Then $f_{0}$ and $f_{1}$ are called homotopic relative $A$, denoted $f_{0} \simeq f_{1}($ rel $A)$ if there exists a map $H:[0,1] \times Y \rightarrow X$ with $f_{0}(y)=H(0, y), f_{1}(y)=$ $H(1, y)$ and $H(s, a)=f_{0}(a)=f_{1}(a)$ for all $a \in A$. The map $H$ is then called a homotopy relative $A$. A space $X$ is called contractible if id $: X \rightarrow X$ is homotopic to a constant map $X \rightarrow X, x \mapsto x_{0}$ for some $x_{0} \in X$.

(iv) Two based loops $\gamma_{0}, \gamma_{1} \in \Omega\left(X, x_{0}\right)$ are called homotopic if they are homotopic relative $\{0,1\}$. (Notice the abuse of language. If two loops are homotopic in the sense of (iii) we will call them freely homotopic.) The set of homotopy classes of loops based at $x_{0}$ is denoted $\pi_{1}\left(X, x_{0}\right)$.

Exercise 2.3.2 (i) Show that composition descends to a well-defined map $*: \pi_{1}\left(X, x_{0}\right) \times \pi_{1}\left(X, x_{0}\right) \rightarrow \pi_{1}\left(X, x_{0}\right)$.

(ii) Show that with this multiplication $\left(\pi_{1}\left(X, x_{0}\right)\right.$, *) is a group.

Definition 2.3.3 The group $\left(\pi_{1}\left(X, x_{0}\right), *\right)$ is called the fundamental group of $X$ at $x_{0}$.

isomorphism

Exercise 2.3.4 Let $x_{0}, x_{1} \in X$ and let $\gamma:[0,1] \rightarrow X$ with $\gamma(0)=x_{0}, \gamma(1)=$ $x_{1}$. Show that

$$
\pi_{1}\left(X, x_{0}\right) \rightarrow \pi_{1}\left(X, x_{1}\right), \quad[\alpha] \mapsto\left[\gamma * \alpha * \gamma^{-}\right]
$$

is an isomorphism, where composition of paths is defined as composition of loops above and $\gamma^{-}(t):=\gamma(1-t)$. Conclude that the isomorphism type of the fundamental group of a arcwise connected space $X$ does not depend on the base point. 
Definition 2.3.5 An arcwise connected space $X$ is called simply-connected if $\pi_{1}\left(X, x_{0}\right)$ is trivial for some (and hence any) $x_{0} \in X$.

In some cases the fundamental group can be computed directly:

Exercise 2.3.6 (i) Show that any contractible space (in particular the disc) is simply-connected.

(ii) Let $T$ be a topological space, $S$ be a subset of $T$. Let $D$ be the unit disc and $\mathbb{T}^{1}$ its boundary. Let finally $\varphi: \mathbb{T}^{1} \rightarrow S$ be a homeomorphism, show that there is a surjective group homomorphism $\pi_{1}(T) \rightarrow \pi_{1}\left(T \cup_{\varphi} D\right)$.

(iii) Using (i) and (ii), show that the 2-sphere is simply-connected.

\section{Covering and fundamental group}

In general, direct computations of the fundamental group using the above definition are rarely possible. Usually the fundamental group of a space is computed from a realisation of the space as a nice quotient of a simply-connected one. For this we introduce the following notions:

Definition 2.3.7 Let $\Gamma$ be a (discrete) group acting on a space $M$. Then the action is called free if it has no fixed point, in other words $\gamma \cdot m=m$ for some $\gamma \in \Gamma, m \in M$ implies $\gamma=e$. It is called properly if for any compact set $K \subset M$ the set

$$
\{\gamma \in \Gamma \mid \gamma K \cap K \neq \emptyset\}
$$

is finite.

Then the following is a weak version of the main theorem of covering theory:

Theorem 2.3.8 [COVERING TheOrem] Let $M$ be a locally connected, simply connected space $M$. Let $x_{0}$ be any point in $M$. For any, $x$ let $\gamma(x)$ be a path from $x_{0}$ to $x$.

Let $\Gamma$ be a group acting freely and properly on $M$ and $\pi$ be the projection from $M$ to $\Gamma \backslash M$. Then the map

$$
\Gamma \rightarrow \pi_{1}\left(\Gamma \backslash M, \pi\left(x_{0}\right)\right), \quad \gamma \mapsto[\pi(\gamma(x))],
$$

is a group isomorphism and does not depend on the choices of the paths $\gamma(x)$. In particular,

$$
\pi_{1}(\Gamma \backslash M,[x]) \cong \Gamma
$$




\subsubsection{Cayley graph and presentation complex}

Using Theorem 2.3.8, there is a way to realise any given group $\Gamma$ as the fundamental group of a topological space. More precisely given a group $\Gamma$, we shall construct a simply-connected space $C_{2}(\Gamma)$ on which $\Gamma$ acts freely and properly. Then the theorem implies that $\pi_{1}\left(\Gamma \backslash C_{2}(\Gamma), x\right) \cong \Gamma$ for any $x \in \Gamma \backslash C_{2}(\Gamma)$.

For the construction of $C_{2}(\Gamma)$ we proceed as follows:

start with a presentation of $\Gamma$ of the form

$$
\Gamma=\left\langle c_{1}, \ldots, c_{k} \mid R_{1}=\cdots=R_{p}=e\right\rangle,
$$

where $R_{1}, \cdots R_{p}$ are certain words on $c_{1}, \ldots, c_{k}$. By adding further generators and relations if necessary we may assume that there is a fixed point free involution denoted $c_{j} \mapsto c_{\bar{j}}$ for $j=1, \ldots, k$ on the set of generators such that $c_{\bar{j}}$ is inverse to $c_{j}$ as an element of $\Gamma$. Let us call such a presentation admissible.

Definition 2.3.9 Assume that we are given an admissible presentation of the group $\Gamma$, then the Cayley graph of $\Gamma$ with respect to the given presentation is $C(\Gamma)=(V, E)$, where

- the set of vertices is given by $V=\Gamma$

- the vertex $\eta$ is joined to the vertex $\gamma$ by an oriented edge (labelled by the generator $c_{i}$ ) if $\eta=\gamma c_{i}$.

- the reversing orientation involution on the set of oriented edges is the involution that associates to the edge joining $\eta$ to $\gamma$ labelled by $c_{i}$, the edge from $\gamma$ to $\eta$ labelled by $c_{i}^{-1}$.

Example 2.3.10 Let $F$ be a free group over a set $X$. Then $F$ has a presentation

$$
F=\left\langle\{x\}_{x \in X} \mid \emptyset\right\rangle
$$

In order to bring this into an admissible presentation, we add one generator $x^{-1}$ for each $x \in X$ and obtain a new presentation

$$
F=\left\langle\left\{x, x^{-1}\right\}_{x \in X} \mid x x^{-1}=x^{-1} x=e\right\rangle .
$$

The vertices of the Cayley graph are labelled by reduced words over the set of generators $\left\{x, x^{-1}\right\}_{x \in X}$, where a reduced word is a word in these letters without subwords of the form $\left(x, x^{-1}\right)$ for $x \in X$. There is a geometric edge labelled by $\left(x, x^{-1}\right)$ for $x \in X$ between $\left(x_{1}, \ldots, x_{k}\right)$ and $\left(x_{1}, \ldots, x_{k}, x\right)$, where $x_{1}, \ldots, x_{k} \in X$. The corresponding Cayley graph $C(F)$ is a tree and hence its geometric realisation $|C(\Gamma)|$ is simply-connected. 
In general the geometric realisation of a Cayley graph need not be simplyconnected. However, it is easy to compute the fundamental group using an admissible presentation:

Exercise 2.3.11 Let $\Gamma=\left\langle c_{1}, \ldots, c_{k} \mid R_{1}=\cdots=R_{p}=e\right\rangle$ be an admissible presentation of a group $\Gamma$ and let $F$ be the free group on generators $c_{1}, \ldots, c_{k}$. Denote by $R$ the subgroup of $F$ normally generated by the relations $R_{1}, \ldots, R_{p}$.

(i) Show that $F$ and hence $R$ act freely and properly on the geometric realisation $|C(F)|$ of the Cayley graph of the free group $F$ with respect to the given presentation.

(ii) Show that $|C(\Gamma)| \cong|C(F) / R|$ and conclude that $\pi_{1}(|C(\Gamma)|) \cong R$.

Let $R_{j}, 1 \leq j \leq p$ be a relation in $\Gamma$, written as $R_{j}=c_{i_{1}} \cdots c_{i_{k}}$ where the $c_{j}$ are generators. Then any $\gamma \in \Gamma$ satisfies

$$
\gamma c_{i_{1}} \cdots c_{i_{k}}=\gamma
$$

thus there exists a loop in $C(\Gamma)$ starting from $\gamma$ consisting of edges labelled by $c_{i_{1}}, \cdots, c_{i_{k}}$ in precisely that ordering. In the geometric realisation of $C(\Gamma)$ these form a circle and we can glue a disc along this circle to $|C(\Gamma)|$ for any such loop. The resulting space is called the presentation 2-complex of $\Gamma$ with respect to the given presentation and denoted $C_{2}(\Gamma)$. The case of the group $\mathbb{Z}^{2}$ with the admissible presentation

$$
\left\langle a, b, a^{-1} b^{-1} \mid a b a^{-1} b^{-1}=a a^{-1}=b b^{-1}=e\right\rangle
$$

is depicted in Figure 2.18.

Exercise 2.3.12 Use the special form of $\pi_{1}(C(\Gamma))$ derived in Exercise 2.3.11 to prove that the image of $\pi_{1}(|C(\Gamma)|)$ in $\pi_{1}\left(C_{2}(\Gamma)\right)$ is trivial. (Hint: It suffices to prove that the generators $g R_{i} g^{-1}$ of $\pi_{1}(|C(\Gamma)|)$ are mapped to contractible loops!) Then apply Exercise 2.3.6 to conclude that $C_{2}(\Gamma)$ is simply-connected.

Observe that the action by left multiplication of $\Gamma$ on itself induces an action on $C(\Gamma)$, in other words an action of the set of vertices which preserves adjacency. This in turn induces an action of $\Gamma$ on the geometric realisation $|C(\Gamma)|$. This action extends to $C_{2}(\Gamma)$. Indeed, the action of $\Gamma$ maps each loop to another loop and the corresponding homeomorphism of circles in the geometric realisation extend to the discs bounded by these circles as in the proof of Proposition 2.2.10.

Proposition 2.3.13 The action of $\Gamma$ on $C_{2}(\Gamma)$ is free and properly discontinuous. Thus

$$
\pi_{1}\left(\Gamma \backslash C_{2}(\Gamma)\right) \cong \Gamma .
$$

Exercise 2.3.14 Prove Proposition 2.3.13. 


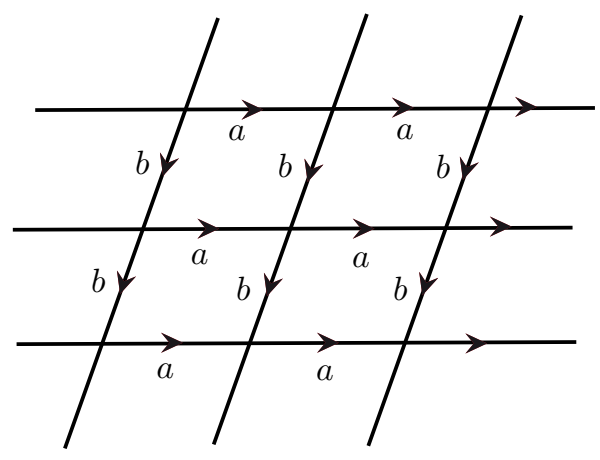

Figure 2.18: A presentation 2-complex of $\mathbb{Z}^{2}$

\subsubsection{Classification of surfaces II: Uniqueness}

We apply Proposition 2.3.13 to prove the following uniqueness part of the classification:

Theorem 2.3.15 The fundamental group of the surface $S_{g}$ is given by

$$
\pi_{1}\left(S_{g}\right) \cong\left\langle a_{1}, b_{1}, \ldots, a_{g}, b_{g} \mid \prod_{i=1}^{g} a_{i} b_{i} a_{i}^{-1} b_{i}^{-1}=e\right\rangle .
$$

These groups are non-isomorphic for different choices of $g$.

Proof: For $g=0$ the theorem predicts $\pi_{1}\left(S^{2}\right)=\{e\}$ which was proved in Exercise 2.3.6. Thus let us assume $g \geq 1$ and abbreviate

$$
A_{g}:=\left\langle a_{1}, b_{1}, \ldots, a_{g}, b_{g} \mid \prod_{i=1}^{g} a_{i} b_{i} a_{i}^{-1} b_{i}^{-1}=e\right\rangle .
$$

Adding the inverse of the generators this presentation, we construct the associated Cayley graph and presentation 2-complex. Then $\Gamma \backslash C_{2}(\Gamma)$ is homeomorphic to gluing a disc whose boundary is labelled by $a_{1}, b_{1}, a_{1}^{-1}, b_{1}^{-1}, \ldots, b_{g}^{-1}$ according to this labelling. We have seen before that this is a description of $S_{g}$ and thus the identity $\pi_{1}\left(S_{g}\right)=A_{g}$ follows from Proposition 2.3.13. The last statement of the theorem follows from the fact that

$$
\operatorname{Hom}\left(A_{g}, \mathbb{R}\right) \cong \mathbb{R}^{2 g} .
$$

Q.E.D. 
A group isomorphic to $A_{g}:=\pi_{1}\left(S_{g}\right)$ will be called a surface group. By Theorem 2.2.13 and Theorem 2.3.15 we can associate to every compact oriented surface a unique non-negative integer $g$ in such a way that $\pi_{1}(S) \cong A_{g}$. This integer is the genus of $S$. Using this terminology, we summarise the classification by saying that any compact oriented surface is uniquely determined by its genus and conversely every non-negative integer occurs as the genus of a surface.

\subsection{Combinatorial versions of the fundamen- tal group}

We shall need other versions of the fundamental group: first we will explain how to compute the fundamental group of the surface out of a filling ribbon graph, secondly we will explain how to build the fundamental group using covers and coverings.

\subsubsection{A combinatorial description using a ribbon graph}

In this section, we relate the filling ribbon graph of a surface and its fundamental group. For the first part of the classification we have used the existence of filling ribbon graphs. These ribbon graphs are not unique but they can be deformed into a filling ribbon graph of type $\Gamma_{g}$. Using different ribbon graphs one can compute the fundamental group of the surface in different ways. This gives rise to different presentations of surface groups.

Let $\Gamma$ be a ribbon graph. Denote by $E, V$ and $F$ respectively the set of its edges, vertices and faces.

Definition 2.4.1 [COMBInAtorial LOOPS AND PATHS] $A$ discrete path is a finite sequence $\left(e_{1}, \ldots, E_{n}\right)$ so that $e_{i}^{+}=e_{i+1}^{-}$. The starting point of such a path is $e_{1}^{-}$, its end point is $e_{n}^{+}$. A path is a discrete loop if its end and starting points coincide. A loop is based at the vertex $v_{0}$ if $v_{0}$ is its starting point. The inverse path of $e=\left(e_{1}, \ldots, e_{n}\right)$ is $\bar{e}:=\left(\bar{e}_{n}, \ldots, \bar{e}_{1}\right)$

We may now define the ribbon fundamental group . Let $F(E)$ be the free group generated by the edges; observe that every path defines an element of $F(E)$. Let $L_{\Gamma}^{v_{0}}$ be the image of the loops based at $v_{0}$ in $F(E)$. Observe that $L_{v_{0}}$ is a subgroup of $F(E)$. Let $R_{\Gamma}^{v_{0}}$ be the subgroup of $L_{\Gamma}^{v_{0}}$ normally generated by faces $f=\left(e_{1}, \ldots, e_{k}\right)$.

Definition 2.4.2 [RIBbon Fundamental GROUP] 
- The group $L_{\Gamma}^{v_{0}}$ is the group of loops based at $v_{0}$.

- The group $R_{\Gamma}^{v_{0}}$ is the group of homotopically trivial loops.

- The ribbon fundamental group is

$$
\hat{\pi}_{1}\left(\Gamma, v_{0}\right)=L_{\Gamma}^{v_{0}} / R_{\Gamma}^{v_{0}}
$$

- Two path e and $f$ with the same starting and end points are homotopic if the loop e. $\bar{f}$ belongs to the group of homotopically trivial loops.

Observe that if $\Gamma^{\prime}$ is a subgraph of $\Gamma$ we have a natural surjection from $\hat{\pi}_{1}\left(\Gamma, v_{0}\right)$ to $\hat{\pi}_{1}\left(\Gamma, v_{0}\right)$. Moreover if $\Gamma$ is a filling graph embedded in a surface $S$, we have a natural map $\hat{i}$ from $\hat{\pi}_{1}\left(\Gamma, v_{0}\right)$ to $\pi_{1}\left(S, v_{0}\right)$ which is build by sending every combinatorial loop to its geometric realisation.

Theorem 2.4.3 Let $S$ be a surface and $\Gamma$ a filling ribbon graph in $S$. The natural map $\hat{i}$ described in the previous definition is an isomorphism from the ribbon fundamental group to the fundamental group of the surface.

The proof is essentially the same as for Theorem 2.3.15. We leave the details to the reader.

\subsubsection{A combinatorial description of the fundamental group using covers}

\section{The fundamental group of a cover}

We want to define fundamental groups through covers. Recall that a open cover of $X$ is a family of open sets whose reunion is $X$. Since we shall only consider open covers, we will usually omit the word open when we speak of open cover.

Definition 2.4.4 [PATH] Let $\mathcal{U}=\{U\}_{i \in I}$ be a cover of a space $X$. A path on the cover based at $i_{0}$ is an element $i_{0} \ldots i_{p}$ of the free group $F_{I}$ generated by $I$ such that $U_{i_{j}} \cap U_{i_{j+1}}=\emptyset$. A loop is a path $\left(i_{0}, \ldots, i_{p}\right)$ so that $i_{0}=i_{p}$. A trivial loop is a loop of the form $\left(j_{0}, \ldots j_{p}, I, J, K, j_{p}, \ldots j_{0}\right)$ where $U_{I} \cap U_{J} \cap U_{k} \neq \emptyset$.

Definition 2.4.5 [Fundamental Group] Let $\mathcal{U}=\{U\}_{i \in I}$ be a cover of $M$. The fundamental group $\pi_{1}^{\mathcal{U}}(M)$ of the cover is the group of loops based at $i_{0}$ - considered as a subgroup of the free group generated by I - up to the group generated by trivial loops. 
In other words, the fundamental group of a cover $\mathcal{U}=\{U\}_{i \in I}$ is the fundamental group of the 2-complex, whose set of vertices is the set $I$, set of edges is the set of intersecting open sets, and set of faces is the set of triples of intersecting open sets.

We observe that if $\mathcal{V}=\left\{V_{j}\right\}_{j \in J}$ is a refinement of $\mathcal{U}$, that is a cover such there exists a map $f: J \rightarrow I$ so that $V_{j} \subset U_{f(j)}$, then we have a natural map

$$
\hat{f}: \pi_{1}^{\mathcal{V}}(S) \rightarrow \pi_{1}^{\mathcal{U}}(S) .
$$

Exercise 2.4.6 Show that the map above does not depend on the choice of the map $f$. Show that the map above is surjective provided the open sets $U_{i}$ are connected.

We shall admit the following result which relies on Van Kampen Theorem [Hat02].

Theorem 2.4.7 Let $\left\{U_{i}\right\}$ be a cover such that the $U_{i}$ are simply connected and $U_{i} \cap U_{j}$ are connected. For every $i$, let $x_{i}$ be an element of $U_{i}$. For every pair $\left(U_{i}, U_{j}\right)$ of intersecting open sets, we choose a path $\gamma_{i j}$ joining $x_{i}$ to $x_{j}$. We associate to every loop on the covering $i_{0} \ldots i_{n}$ where $i_{n}=i_{0}$, the loop $\gamma_{i_{0} i_{1}} \star \ldots \gamma_{i_{n-1} i_{n}}$. This construction is an isomorphism from $\pi_{1}^{\mathcal{U}}(M)$ to $\pi_{1}(M)$.

It follows from this result that if $X$ admits a cover by simply connected sets with connected intersections, then we have a well defined surjective map from $\pi_{1}(M)$ to $\pi_{1}^{\mathcal{U}}(M)$ for any cover $\mathcal{U}$ of $X$.

Exercise 2.4.8 Reprove Theorem 2.4.3 using Theorem 2.4.7.

\section{Covers and covering maps}

A Galois cover of group $G$ is a map $\pi: \Sigma \rightarrow S$, where $G$ acts properly discontinuously without fixed points on $\Sigma, S=\Gamma \backslash \Sigma$ and $\pi$ the projection. A trivialising cover is a cover $\mathcal{U}=\left\{U_{i}\right\}_{i \in I}$ of $S$ so that for every $i$,

$$
\pi^{-1}\left(U_{i}\right)=\bigsqcup_{\gamma \in G} \gamma V_{i}
$$

We can relate covers and coverings as follows. Let $\pi: \Sigma \rightarrow S$ be a Galois cover of group $G$. Let $\mathcal{U}$ be a trivialising cover of $S$ as above so that every $U_{i}$ is connected. Let finally $\hat{\mathcal{U}}=\left\{g V_{i}\right\}_{i \in I, g \in G}$.

Proposition 2.4.9 Let $\pi: \Sigma \rightarrow S$ be a Galois cover. Let $\mathcal{U}$ be a trivialising cover of $S$ for $\pi$, so that the intersection of two open sets is connected. Then, we have a short exact sequence

$$
0 \rightarrow \pi_{1}^{\hat{\mathcal{U}}}(\Sigma) \rightarrow \pi_{1}^{\mathcal{U}}(S) \rightarrow G .
$$


Proof: The hypothesis on $\mathcal{U}=\left\{U_{i}\right\}_{i \in I}$ yields that we can lift uniquely every path $\mathcal{U}$-path to a $\hat{\mathcal{U}}$-path, which maps trivial loops to trivial loops. Hence we obtain the short exact sequence

$$
0 \rightarrow \pi_{1}^{\hat{\mathcal{U}}}(\Sigma) \rightarrow \pi_{1}^{\mathcal{U}}(S) \rightarrow G
$$

Q.E.D.

\subsection{Cohomology of Surfaces}

This whole section is a warm up for the next chapter. We shall give - and prove - more general statements in this next chapter. However, we specialise in this section to the standard situation of - untwisted - cohomology groups so that the reader can parallel this construction to the more general one.

\subsubsection{De Rham cohomology}

In this section we shall introduce and compute the cohomology groups of a surface $S$. These groups have several constructions, in this section we shall use the existence of a differentiable structure on $S$ and construction the de Rham cohomology groups using differential forms.

\section{Derivative, tangent and cotangent spaces}

Let $S$ be a closed surface, in other words a compact connected smooth 2manifold. We denote by $\mathrm{T}_{m} S$ the tangent space to $S$ at the point $m$. The dual space $\mathrm{T}_{m}^{*} S$ is called the cotangent space of $S$ at $m$. The cotangent space $\mathrm{T}_{m}^{*} M$ is described as follows. Let $C_{0}^{0}(M, m)$ be the vector space of functions that vanishes at a point $m$ in $M$. We say two functions $f$ and $g$ of $C_{0}^{0}(M, m)$ have the same derivative at $m$ if we can write

$$
f-g=\sum_{i=1}^{i=n} \varphi_{i} \psi_{i}
$$

where $\psi_{i}$ and $\varphi_{i}$ belong to $C_{0}^{0}(M, m)$. We observe that this notion coincide with the classical one when $M=\mathbb{R}^{n}$ that is $f$ and $g$ have the same derivative at $m$ if and only if $\mathrm{d} f(m)=\mathrm{d} g(m)$.

\section{Differential forms}

Informally speaking, a differential $k$-form - or shortly a $k$-form - is a map which assigns to each point $m \in S$ a vector $\omega_{m} \in \bigwedge^{k} \mathrm{~T}_{m}^{*} S$ for some $k \geq 0$, such that furthermore $\omega_{m}$ varies "smoothly" (see below). 
Example 2.5.1 1. By convention, $\bigwedge^{0} \mathrm{~T}_{m}^{*} S:=\mathbb{R}$ and thus a (smooth) 0form is just a (smooth) function.

2. If $f$ is a smooth function on $S$ then its differential $\mathrm{d}_{x} f$ is a linear form on tangent space and thus $\mathrm{d} f$ is a 1 -form.

If $U$ is a contractible open coordinate chart with coordinates $x_{1}, \ldots, x_{n}$ then the differentials $\mathrm{d} x_{j}$ span the differential forms as an algebra. This means, that any $k$-form on $U$ can be written as

$$
\left.\omega\right|_{U}=\sum_{I=\left(i_{1}, \ldots, i_{k}\right)} \omega_{I} \mathrm{~d} x_{i_{1}} \wedge \cdots \wedge \mathrm{d} x_{i_{k}}
$$

with $\omega_{I}: U \rightarrow \mathbb{R}$. With this at hand, we can make the notion of smooth $k$-form rigorous: a $k$-form $\omega$ is smooth, if for every point $m \in S$ there exists a contractible open coordinate chart around $m$ such that if $\left.\omega\right|_{U}$ is written as above the functions $\omega_{I}$ are smooth. It is easy to see that this does not depend on the choice of chart. We will only consider smooth forms and thus we reserve both the terms differential form and $k$-form for smooth $k$-forms.

We denote by $\Omega^{k}(S)$ to denote $k$-forms on $S$. Note that $\Omega^{k}(S)=\{0\}$ for $k>\operatorname{dim} S=2$. It will be convenient for us to define $\Omega^{k}(S)=\{0\}$ also for $k<0$. Then

$$
\Omega^{\bullet}(S):=\bigoplus_{k \in \mathbb{Z}} \Omega^{k}(S)
$$

becomes a $\mathbb{Z}$-graded $C^{\infty}(S)$-algebra, in other words it is a vector space and module over $C^{\infty}(S)$ and the wedge product defines an algebra structure which maps $\Omega^{k}(S) \times \Omega^{l}(S)$ into $\Omega^{k+l}(S)$. All these definitions make sense for arbitrary manifolds.

\section{Support, pullbacks}

The support of a differential form $\omega$ to be the closure of those points $x$ for which $\omega$ can be expressed in a neighbourhood $U$ of $x$ in the standard form

$$
\left.\omega\right|_{U}=\sum_{I=\left(i_{1}, \ldots, i_{k}\right)} \omega_{I} \mathrm{~d} x_{i_{1}} \wedge \cdots \wedge \mathrm{d} x_{i_{k}}
$$

in such a way, that at least one of the coefficient functions $\omega_{I}$ does not vanish at $x$. Then $\omega$ is called compactly supported, if its support is a compact subset of $S$. We denote the compactly supported $k$-forms on $S$ by $\Omega_{c}^{k}(S)$. Of course $\Omega^{k}(S)=\Omega_{c}^{k}(S)$ if and only if $S$ is compact. 
If $S_{1}$ and $S_{2}$ are surfaces or, more generally, manifolds and $f: S_{1} \rightarrow S_{2}$ is smooth then we can define a pullback map

$$
f^{*}: \Omega^{\bullet}\left(S_{2}\right) \rightarrow \Omega^{\bullet}\left(S_{1}\right)
$$

which for $\omega \in \Omega^{k}\left(S_{2}\right)$ and tangent vectors $X_{1}, \ldots, X_{k}$ at some point $m$ of $S_{1}$ is given by

$$
f^{*} \omega_{m}\left(X_{1}, \ldots, X_{k}\right):=\omega_{f(m)}\left(\mathrm{\top} f\left(X_{1}\right), \ldots, \mathrm{\top} f\left(X_{k}\right)\right)
$$

\section{Integration}

Differential forms can be integrated on oriented manifolds. Let us consider the case of an open subset $U$ of $\mathbb{R}^{n}$. Let $\omega=f \mathrm{~d} x_{1} \wedge \cdots \wedge \mathrm{d} x_{n} \in \Omega^{n}(U)$ be with compact support. Then we define

$$
\int_{U} \omega:=\int_{U} f \mathrm{~d} x_{1} \wedge \cdots \wedge \mathrm{d} x_{n}:=\int_{U} f \mathrm{~d} \lambda^{n}\left(x_{1}, \ldots, x_{n}\right),
$$

where $\lambda^{n}$ is the $n$-dimensional Lebesgue measure.

If $\varphi$ is any diffeomorphism then

$$
\int_{U} \varphi^{*} \omega= \pm \int_{\varphi(U)} \omega
$$

where the sign is positive if $\varphi$ preserves orientation and negative if $\varphi$ reverses the orientations. As a consequence of this sign irritation we can only define integration for oriented manifolds:

Proposition 2.5.2 If $M$ is an oriented n-dimensional manifold then there exists a unique linear map

$$
\Omega_{c}^{n}(M) \rightarrow \mathbb{R}, \quad \omega \rightarrow \int_{M} \omega
$$

such that if $\omega$ is supported in a chart $(U, x)$ with $x$ orientation-preserving then

$$
\int_{M} \omega=\int_{x(U)}\left(x^{-1}\right)^{*} \omega .
$$

Convention 2.5.3 Suppose $N \subset M$ is a $k$-dimensional oriented submanifold and $\iota: N \rightarrow M$ denotes the inclusion. Then we can integrate any $k$-form $\omega \in \Omega^{k}(M)$ over $N$. We will use a slight abuse of notation and denote this by

$$
\int_{N} \omega:=\int_{N} \iota^{*} \omega .
$$




\section{Exterior derivatives}

The crucial result which helps us defining cohomology is the following proposition.

Proposition 2.5.4 There exists a unique family of linear operators

$$
\mathrm{d}^{k}: \Omega^{k}(M) \rightarrow \Omega^{k+1}(M),
$$

with the following properties:

(i) $\mathrm{d}^{0}$ coincides with the usual differential $C^{\infty}(M) \rightarrow \Omega^{1}(M)$.

(ii) $\mathrm{d}^{k}\left(f \mathrm{~d}^{k-1} \alpha\right)=\mathrm{d}^{0} f \wedge \mathrm{d}^{k-1} \alpha$ for $f \in C^{\infty}(M), \alpha \in \Omega^{k-1}(M)$.

The proof follows by a simple recursion and and will not be reproduced here. Uniqueness follows from the fact that locally any $\mathrm{k}$ form is a sum of expressions of the form $f \mathrm{~d}^{k-1} \alpha$ and existence is proved using a smooth partition of unity. Note that (ii) immediately implies

$$
\mathrm{d}^{k} \mathrm{~d}^{k-1} \equiv 0 .
$$

This allows the following definition.

Definition 2.5.5 A $k$-form $\omega \in \Omega^{k}(M)$ is closed if $\mathrm{d}^{k} \omega=0$ and exact if there exists $\alpha \in \Omega^{k-1}$ with $\omega=\mathrm{d}^{k-1} \alpha$.

Then define the $k$-th de Rham cohomology group of $M$ is

$$
H_{d R}^{k}(M):=\operatorname{kerd} \mathrm{d}^{k} / \operatorname{Im~d}^{k-1}
$$

. In other words the quotient of the space of closed forms by the set of exact forms. The dimension

$$
\beta_{k}:=\operatorname{dim} H_{d R}^{k}(M)
$$

is called the $k$-th Betti number of $M$.

More generally, recall that a complex of vector spaces or modules is a family $C^{\bullet}$ of vector spaces $C^{k}$ together with linear maps $\mathrm{d}^{k}$ from $C^{k} \rightarrow C^{k+1}$ so that $\mathrm{d}^{k+1} \circ \mathrm{d}^{k}=0$. Thus, using this language, the differential forms on a manifold together with the differential form form a complex called the de Rham complex

De Rham cohomology as strong links with homotopy:

Lemma 2.5.6 [POINCARÉ LEMMA] If $U$ is a contractible manifold, then every closed form is exact. In other words, $H_{d R}^{k}(U)=0$ for all $k>0$.

The Poincaré Lemma is a corollary of the fundamental 
Lemma 2.5.7 [Номотору Lemma] Let $M$ be a manifold and $F_{0}$ and $F_{1}$ be two smooth homotopic maps from $M$ to $N$. Let $\beta$ be a closed form in $N$ then

$$
F_{0}^{*} \beta-F_{1}^{*} \beta,
$$

is exact.

If one has a covering of a space by contractible submanifolds (which always exists for manifolds) then one can use Poincaré's Lemma in order to reduce the computation of $H_{d R}^{k}(M)$ to a combinatorial counting process. This is called the Mayer-Vietoris argument and will be discussed and applied below.

The relation between the de Rham differential of a manifold and integration is described by the following generalisation of the fundamental theorem of calculus:

Theorem 2.5.8 [STOKES FORMULA]

Let $M$ be an oriented manifold with boundary $\partial M$. Then

$$
\int_{\partial M} \omega=\int_{M} \mathrm{~d} \omega
$$

In particular, if $\partial M=\emptyset$ then the integral of a differential form only depends on its cohomology class.

\section{Hurewicz Theorem}

We can now the first de Rham cohomology group with the fundamental group as follows.

Theorem 2.5.9 [Hurewicz TheOREM] Let $M$ be a connected manifold. Let $h$ be the mapping defined by

$$
\left\{\begin{aligned}
H^{1}(M, \mathbb{R}) & \rightarrow \operatorname{Hom}\left(\pi_{1}(M), \mathbb{R}\right), \\
{[\omega] } & \mapsto h_{[\omega]},
\end{aligned}\right.
$$

where for every curve $\gamma: \mathbb{T}^{1} \rightarrow M$

$$
h_{[\omega]}(\gamma)=\int_{\mathbb{T}^{1}} \gamma^{*} \omega
$$

Then, $h$ is an isomorphism.

Exercise 2.5.10 Use Stokes Formula and the Homotopy Lemma to show that the above statement actually makes sense. Then prove Hurewicz Theorem. 
Thus we can identify

$$
H_{d R}^{1}(M, \mathbb{R}) \cong \operatorname{Hom}\left(\pi_{1}(M), \mathbb{R}\right) \cong \operatorname{Rep}\left(\pi_{1}(M), \mathbb{R}\right),
$$

where for the last identity we used that $\mathbb{R}$ is abelian and thus acts trivially bu conjugation. Thus the first de Rham cohomology group of a manifold is our first example of a representation variety. Moreover, and this is more important for us in the sequel, we can identify $H_{d R}^{1}(M, \mathbb{R})$ with the tangent space of the representation variety at some fixed basepoint. We will see later on, that in the case of a general representation variety the tangent spaces (at the smooth points) can still be identified with a twisted version of de Rham cohomology. This is the motivation for us to study de Rham cohomology at this place.

\section{Extension of differential forms}

We close this paragraph with an auxiliary result on extendability of differential forms:

Lemma 2.5.11 [EXTENSION LEMma] Let $M$ be a manifold, $V, U$ open subsets of $M$ with $\bar{V} \subset U$. If $\omega=\mathrm{d} \alpha$ on $U$ for some $\alpha \in \Omega^{k}(U)$ then there exists $\widetilde{\alpha} \in \Omega^{k}(M)$ such that $\left.\omega\right|_{V}=\left.\mathrm{d} \widetilde{\alpha}\right|_{V}$.

The proof uses the existence of a smooth bump function with support in $U$ which is 1 on $\bar{V}$ and 0 outside $U$ and is left to the reader.

\subsubsection{Ribbon graphs and cohomology}

When $S$ is a compact orientable surface, we will describe and compute in a combinatorial way the cohomology using ribbon graphs rather than differential forms.

To explain this approach, let $S$ be a surface and let $\Gamma$ be a filling ribbon graph for $S$. As $S$ is uniquely determined by $\Gamma$, it is obvious that the de Rham cohomology of $S$ is also uniquely determined by $\Gamma$. It is less obvious, however, that there is an explicit way to compute the cohomology of $S$ using $\Gamma$. We will describe such a method presently. We will consider a generalisation of this method in great detail later on when we discuss twisted cohomology. Therefore we only explain the basic constructions here and do not provide any proofs. The reader is invited to specialise the more general proofs given in Section 4.1.7 below to the present case. 
Denote by $V, E, F$ the sets the vertices, edges and faces of the filling ribbon graph $\Gamma$. We define the sets of combinatorial 0 -cochains, 1-cochains and 2cochains of $\Gamma$ by

$$
\begin{aligned}
& C_{\Gamma}^{0}(S):=\{f: V \rightarrow \mathbb{R}\} \\
& C_{\Gamma}^{1}(S):=\{f: E \rightarrow \mathbb{R} \mid f(\bar{e})=-f(e)\}, \\
& C_{\Gamma}^{2}(S):=\{f: F \rightarrow \mathbb{R}\} .
\end{aligned}
$$

We then define differentials

$$
\begin{aligned}
& \mathrm{d}^{0}: C_{\Gamma}^{0}(S) \rightarrow C_{\Gamma}^{1}(S),\left(\mathrm{d}^{0} f\right)(e):=f\left(e^{+}\right)-f\left(e^{-}\right), \\
& \mathrm{d}^{1}: C_{\Gamma}^{1}(S) \rightarrow C_{\Gamma}^{2}(S),\left(\mathrm{d}^{1} \alpha\right)(f):=\sum_{e \in f} \alpha(e) .
\end{aligned}
$$

Then $\mathrm{d}^{1} \circ \mathrm{d}^{0}=0$ because given a face $f$ every vertex occurs as often as the origin of an edge of $f$ as it occurs as a terminus of such an edge. It is convenient to define $C_{\Gamma}^{k}(S):=\{0\}$ for $k \notin\{0,1,2\}$ and $\mathrm{d}^{k}: C_{\Gamma}^{k}(S) \rightarrow C_{\Gamma}^{k+1}(S)$ as the zero map for $k \notin\{0,1\}$. Then $\mathrm{d}^{k+1} \mathrm{~d}^{k} \equiv 0$ for all $k \in \mathbb{Z}$ and thus we may define:

Definition 2.5.12 The group

$$
H_{\Gamma}^{k}(S):=\frac{\operatorname{ker~d}^{k}}{\operatorname{Im~d}^{k-1}}
$$

is called the $k$-th cohomology group of $S$ with respect to $\Gamma$.

Note that by definition, $\operatorname{dim} H_{\Gamma}^{k}(S)<\infty$.

We now relate the cohomology groups of $\Gamma$ to the de Rham cohomology groups of $M$ : We define maps $\iota_{k}: \Omega^{k}(S) \rightarrow C^{k}(\Gamma)$ by

$$
\begin{aligned}
& \iota_{0}: \Omega^{0}(S) \rightarrow C_{\Gamma}^{0}(S), \quad \iota_{0}(f)(v):=f(v) ; \\
& \iota_{1}: \Omega^{1}(S) \rightarrow C_{\Gamma}^{1}(S), \quad \iota_{1}(\alpha)(e):=\int_{e} \alpha ; \\
& \iota_{2}: \Omega^{2}(S) \rightarrow C_{\Gamma}^{2}(S), \quad \iota_{0}(\omega)(f):=\int_{f} \omega .
\end{aligned}
$$

We set $\iota_{k} \equiv 0$ for $k \notin\{0,1,2\}$. Here we have abused notation and identified a vertex, edge or face with its image in the geometric realisation of $\Gamma$, considered as a subset of $S$. For $\alpha \in \Omega^{k}(S)$ we will write $\widehat{\alpha}:=\iota_{k}(\alpha)$. Observe:

Proposition 2.5.13 For any $\alpha \in \Omega^{k}(S)$,

$$
\mathrm{d} \widehat{\alpha}=\widehat{\mathrm{d} \alpha} .
$$

In particular, $\iota_{k}\left(Z^{k}(M)\right) \subset \operatorname{ker} \mathrm{d}^{k}, \iota_{k}\left(B^{k}(M)\right) \subset \operatorname{Imd}^{k}$ and thus $\iota_{k}$ induces a map

$$
\iota_{k}: H_{d R}^{k}(M) \rightarrow H_{\Gamma}^{k}(S) .
$$


Exercise 2.5.14 Derive 2.5.13 from Stokes' formula.

In fact one has:

Theorem 2.5.15 The maps $\iota_{k}: H_{d R}^{k}(S) \rightarrow H_{\Gamma}^{k}(S)$ defined by Proposition 2.5.13 are isomorphisms.

Theorem 2.5.15 has a number of consequences. An obvious one is the following:

Corollary 2.5.16 $\quad$ (i) The (de Rham) cohomology groups of a compact orientable surface are finite-dimensional.

(ii) The groups $H_{\Gamma}^{k}(S)$ do not depend on the choice of filling Ribbon graph which is used in order to define them.

For a manifold with finite-dimensional cohomology spaces we define the Euler characteristic as the alternating sum of its Betti numbers, in other words

$$
\chi(M):=\sum_{i=0}^{\operatorname{dim} M}(-1)^{i} \beta_{i} .
$$

Let us compute the Euler characteristic for compact orientable surfaces. For this we use the following easy algebraic fact:

Lemma 2.5.17 Let $0 \rightarrow C^{0} \stackrel{\mathrm{d}^{0}}{\rightarrow} \ldots \stackrel{\mathrm{d}^{n-1}}{\longrightarrow} C^{n} \rightarrow 0$ be a finite complex of finite-dimensional vector spaces $C^{k}$, in other words $\mathrm{d}^{k+1} \mathrm{~d}^{k}=0$. Let

$$
H^{k}:=\frac{\operatorname{ker} \mathrm{d}^{k}}{\operatorname{Im~d}^{k-1}}
$$

be the associated cohomology groups. Then

$$
\sum_{k=0}^{n}(-1)^{k} \operatorname{dim} H^{k}=\sum_{k=0}^{n}(-1)^{k} \operatorname{dim} C^{k}
$$

We apply this in order to compute the Euler characteristic of a closed surface:

Corollary 2.5.18 If $S \cong S_{g}$ is a compact orientable surface of genus $g$ then its Euler characteristic is given by $\chi(S)=2-2 g$. In particular, a compact oriented surface is uniquely determined by its Euler characteristic. 
Proof: If $\Gamma$ is a filling ribbon graph with vertices $V$, edges $E$ and faces $F$ then

$$
\operatorname{dim} C_{\Gamma}^{0}(S)=|V|, \quad \operatorname{dim} C_{\Gamma}^{1}(S)=\frac{1}{2}|E|, \quad \operatorname{dim} C_{\Gamma}^{2}(S)=|F| .
$$

Thus Lemma 2.5.17 implies

$$
\chi(S)=\operatorname{dim} H_{\Gamma}^{0}(S)-\operatorname{dim} H_{\Gamma}^{1}(S)+\operatorname{dim} H_{\Gamma}^{2}(S)=|V|-\frac{1}{2}|E|+|F| .
$$

If we take for $\Gamma$ the graph $\Gamma_{g}$ then

$$
|V|=|F|=1, \quad \frac{1}{2}|E|=2 g,
$$

and the result follows. Q.E.D.

From this we can finally compute the cohomology of a compact oriented surface:

Corollary 2.5.19 If $S \cong S_{g}$ is a surface of genus $g$ then

$$
H_{d R}^{0}(S) \cong \mathbb{R}, \quad H_{d R}^{1}(S) \cong \mathbb{R}^{2 g}, \quad H_{d R}^{2}(S) \cong \mathbb{R} .
$$

Proof: A function with trivial derivative on a connected manifold is constant and therefore

$$
H_{d R}^{0}(S)=Z_{d R}^{0}(S)=\left\{f \in C^{\infty}(S) \mid \mathrm{d}^{0} f=0\right\} \cong \mathbb{R} .
$$

The result for $H_{d R}^{1}(S)$ follows from the Hurewicz theorem and our computation of the surface group . Then $\chi(S)=2-2 g$ forces $H_{d R}^{2}(S) \cong \mathbb{R}$. Q.E.D.

\section{Application: gluing ribbon graphs}

We had to perform some intellectual gymnastics in order to identify the surface filled by a ribbon graph. It is much more easy by now: we just have to compute its Euler characteristic. This can be used to solve in a simple way Exercises 2.2.8. Here is an exercise as an application

Exercise 2.5.20 Let $\Gamma_{1}$ be a ribbon graph filling a surface of genus $g_{1}$. Let $\Gamma_{2}$ be a ribbon graph filling a surface of genus $g_{2}$. Let $\Gamma_{3}$ be the ribbon graph obtained in the following way: draw a circle such that $\Gamma_{1}$ and $\Gamma_{3}$ are in the two distinct connected component of the complementary, then join this circle to $\Gamma_{1}$ and $\Gamma_{2}$ as in Picture 2.19. Prove that $\Gamma_{3}$ fills a surface of genus $g_{1}+g_{2}$. 


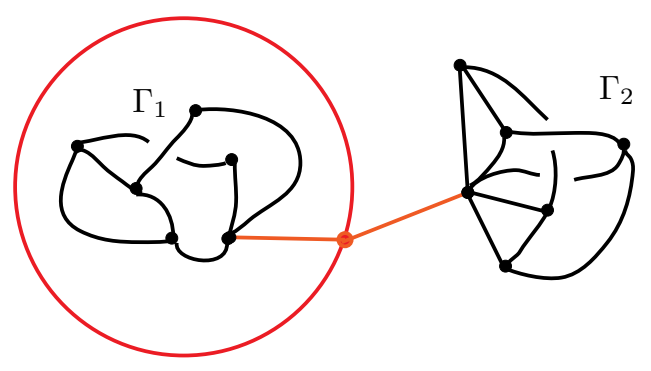

Figure 2.19: Gluing Graphs

\subsubsection{The intersection form}

In the next two subsections we will deal with symplectic structures on vector spaces. Recall the following basic definition:

Definition 2.5.21 Let $V$ be a vector space. A bilinear map $V \times V \rightarrow \mathbb{R}$ is called a linear symplectic form if it is alternating and non-degenerate.

In this subsection we construct a symplectic form on $H_{d R}^{1}(S)$. As explained before, we view this cohomology group as the tangent space of the representation variety $\operatorname{Rep}\left(\pi_{1}(S), \mathbb{R}\right)$ at a base point. Therefore constructing a symplectic form on $H_{d R}^{1}(S)$ is the same as constructing a fibrewise symplectic form on the tangent space of $\operatorname{Rep}\left(\pi_{1}(S), \mathbb{R}\right)$. We will later on generalise this procedure to more general (and in particular, non-abelian) coefficient groups. Again the proofs will be given later on in the more general setting of Section 4.1. Here we will only explain the ingredients for the proof of the following theorem:

Theorem 2.5.22 Let $S$ be a surface. Then the map

$$
i: H_{d R}^{1}(S) \times H_{d R}^{1}(S) \rightarrow \mathbb{R}, \quad([\alpha],[\beta]) \mapsto \int_{S} \alpha \wedge \beta
$$

is a symplectic form.

The map $i$ is called the intersection form of $S$ and it gives a duality on $H_{d R}^{1}(S)$. It is clear from the definition that $i$ is alternating and bilinear. It is less clear, whether $i$ is non-degenerate. To prove non-degeneracy we will use once again ribbon graphs and Theorem 2.5.15. Thus let $S$ be a surface with filling ribbon graph $\Gamma$, and let $\Gamma^{*}$ be its dual. The latter is again a ribbon 


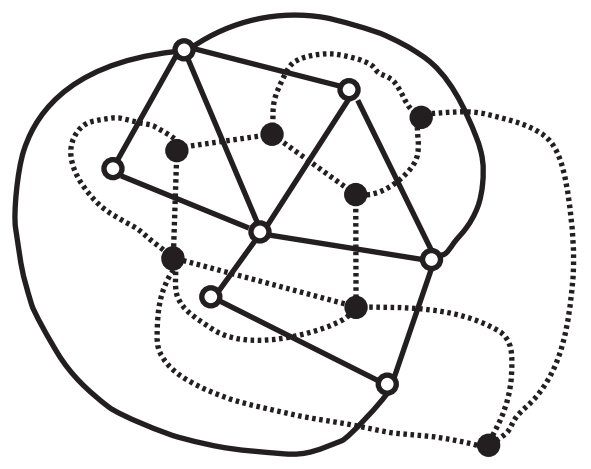

Figure 2.20: A dual graph

graph and it is defined as follows: Denote by $V_{\Gamma}, E_{\Gamma}, F_{\Gamma}$ the vertices, edges and faces of $\Gamma$. We define $V_{\Gamma^{*}}:=F_{\Gamma}, E_{\Gamma^{*}}:=E_{\Gamma}$. If $(e, \bar{e})$ is a geometric edge in $\Gamma$ then $e$ is contained in a unique face $f \in F_{\Gamma}=V_{\Gamma^{*}}$ and $\bar{e}$ is contained in a unique face $\bar{f} \in V_{\Gamma^{*}}$. We then define

$$
\varphi^{*}: E_{\Gamma^{*}} \rightarrow V_{\Gamma^{*}} \times V_{\Gamma^{*}}, \quad \varphi^{*}(e):=(f, \bar{f}) .
$$

Then $\left(V_{\Gamma^{*}}, E_{\Gamma^{*}}, \varphi^{*}\right)$ is a directed graph and the involution $e \leftrightarrow \bar{e}$ turn $\Gamma^{*}$ into a graph. Geometrically $\Gamma^{*}$ is obtained by drawing edges between adjoint faces as in Figure 2.20.

Exercise 2.5.23 Show that $F_{\Gamma^{*}}$ and $E_{\Gamma}$ are in canonical bijection.

Definition 2.5.24 Denote by $C_{\Gamma}^{k}, C_{\Gamma^{*}}^{k}$ the combinatorial cochains of $\Gamma$ and $\Gamma^{*}$ respectively. Then the dual pairing

$$
\langle\cdot \mid \cdot\rangle: C_{\Gamma}^{k} \times C_{\Gamma^{*}}^{2-k} \rightarrow \mathbb{R}
$$

is defined by

$$
\begin{aligned}
& \langle\alpha \mid \beta\rangle:=\sum_{v \in V_{\Gamma}=F_{\Gamma^{*}}} \alpha(v) \beta(v) \quad\left(\alpha \in C_{\Gamma}^{0}, \beta \in C_{\Gamma^{*}}^{2}\right) ; \\
& \langle\alpha \mid \beta\rangle:=\sum_{e \in E_{\Gamma}=E_{\Gamma^{*}}} \alpha(e) \beta(e) \quad\left(\alpha \in C_{\Gamma}^{1}, \beta \in C_{\Gamma^{*}}^{1}\right) ; \\
& \langle\alpha \mid \beta\rangle:=\sum_{f \in F_{\Gamma}=V_{\Gamma^{*}}} \alpha(f) \beta(f) \quad\left(\alpha \in C_{\Gamma}^{2}, \beta \in C_{\Gamma^{*}}^{0}\right)
\end{aligned}
$$

and $\langle\cdot \mid \cdot\rangle \equiv 0$ for $k \notin\{0,1,2\}$. 
Proposition 2.5.25 (i) The dual pairing $\langle\cdot \mid \cdot\rangle: C_{\Gamma}^{k} \times C_{\Gamma^{*}}^{2-k} \rightarrow \mathbb{R}$ satisfies

$$
\langle\mathrm{d} \alpha \mid \beta\rangle=\langle\alpha \mid \mathrm{d} \beta\rangle \quad\left(\alpha \in C_{\Gamma}^{k}, \beta \in C_{\Gamma^{*}}^{1-k}\right) .
$$

(ii) The dual pairing descends to a pairing

$$
\langle\cdot \mid \cdot\rangle: H_{\Gamma}^{k} \times H_{\Gamma^{*}}^{2-k} \rightarrow \mathbb{R}
$$

(iii) The dual pairing is non-degenerate on $H_{\Gamma}^{k} \times H_{\Gamma^{*}}^{2-k}$ for $k \in\{0,1,2\}$.

Recall the definition of the map $\iota_{1}: \Omega^{1}(S) \rightarrow C_{\Gamma}^{1}$ from the last section. As $E_{\Gamma}=E_{\Gamma^{*}}$ and the involution on $E_{\Gamma}$ and $E_{\Gamma^{*}}$ agree we have $C_{\Gamma}^{1} \cong C_{\Gamma^{*}}^{1}$ and thus we have also an isomorphism $\iota_{1}^{*}: \Omega^{1}(S) \rightarrow C_{\Gamma^{*}}^{1}$. We can use these maps to relate the intersection form on $\Omega^{1}(S)$ to the dual pairing. More precisely, we have the following result, which together with Proposition 2.5.25 implies Theorem 2.5.22:

Lemma 2.5.26 Let $\alpha, \beta \in \Omega^{1}(S)$. Then

$$
\int_{S} \alpha \wedge \beta=\left\langle\iota_{1}(\alpha) \mid \iota_{1}^{*}(\beta)\right\rangle .
$$

We prove later on a more general result that contains this lemma as a special case.

\subsection{Comments, references and further read- ings}

Instead of using simplicial description of surfaces, we use the approach by ribbon graphs - which are also called fat graphs. This approach has the disadvantage that it is not straightforwardly generalisable, but on the other hand it is perfectly suited for surfaces. Ribbon graphs gain their mathematical popularity through the work of Penner [Pen92] who introduced a cell decomposition of Riemann moduli space, which was later used in Kontsevich proof of Witten Conjecture [Kon92].

The classification of surfaces argument written in these notes is found in Springer [Spr57] which is still a very nice introduction to Riemann surfaces. Other possible references are Massey, "a basic course in algebraic topology" [Mas91], Stillwell, "Geometry of surfaces" [Sti92].

We presented several aspects of the fundamental groups: first the general definition, then a combinatorial description through ribbon graphs then finally 
through covers and coverings. These presentations are somewhat ad hoc for surfaces but we hope that these various points of views will help the reader see different aspects of a fundamental group: as an abstract group, as defined with generators and relations, as describing coverings and associated to covers.

We chose to present the relation between combinatorial and de Rham cohomology only in the case of surfaces - remark here that the ribbon graph cohomology is slightly different from simplicial cohomology since a ribbon graph decomposition is not a triangulation. Even though this is a very pedestrian approach, we hope that it may help the reader go through the general proofs. The classical Bott-Tu [BT82] is an excellent introduction to de Rham cohomology and its link with combinatorial versions. Even though the categorical and axiomatic approach to these isomorphisms are more powerful, we find important for future use to explain how one can realise these isomorphisms explicitly.

The book by Hatcher [Hat02] is a remarkable introduction to algebraic topology and fundamental groups which should be used as the basic reference for fundamental groups and simplicial homology. 


\section{Chapter 3}

\section{Vector bundles and connections}

In this chapter, we introduce the main objects of our study: vector bundles, connections and representation variety. We give two different point of views on vector bundles, first as a "continuous family" of vector spaces indexed by a topological space, then as cocycles which are of a more combinatorial nature. This allows us in particular to define $G$-structures on vector bundle. After having motivating connections by the need to have explicit isomorphisms depending on choices - between fibres of a vector bundle, we give several description of flat connections using the various languages we have used in parallel for the fundamental group and cohomology: differential geometry, ribbon graphs and coverings.

This allows us to define and give four different models of the representation variety

$$
\operatorname{Hom}\left(\pi_{1}(S), G\right) / G
$$

\subsection{Vector bundles}

This subsection is one more time intended to provide a very brief crash course on the concepts which we shall need. We start with the notion of a vector bundle.

\subsubsection{Definitions}

Informally, a vector bundle is a continuous family of vector spaces indexed by a topological space. The following definition makes this precise.

A vector bundle is a triple $(\mathcal{L}, \pi, M)$, where $\mathcal{L}$ and $M$ are topological spaces called the total space and the total spacerespectively and $\pi: \mathcal{L} \rightarrow M$ is a continuous map satisfying the following two conditions: 
(i) For all $m \in M$ the fibre $\mathcal{L}_{m}:=\pi^{-1}(m)$ is a finite dimensional vector space isomorphic to a given vector space $L$.

(ii) For all $m \in M$ there exists a neighbourhood $U_{m}$ of $m$ and a homeomorphisms

$$
\varphi: \pi^{-1}\left(U_{m}\right) \rightarrow U_{m} \times L,
$$

which restricts to a linear isomorphism $\varphi: \mathcal{L}_{m^{\prime}} \rightarrow\left\{m^{\prime}\right\} \times L$ for any $m^{\prime} \in U_{m}$.

The dimension of $L$ is called the rank of the vector bundle and denoted rk $\mathcal{L}$. If $\operatorname{rk} \mathcal{L}=1$, then $\mathcal{L}$ is called a line bundle. An open subset satisfying property (ii) is called trivialising. By abuse of language we will simply call $\mathcal{L}$ a vector bundle (over $M$ ). A section of $(\mathcal{L}, \pi, M)$ is a continuous map $\sigma: M \rightarrow \mathcal{L}$ such that $\sigma(m) \in \mathcal{L}_{m}$. We denote by $\Gamma(\mathcal{L})$ the space of sections of $(\mathcal{L}, \pi, M)$. Let $\mathcal{L}$ and $\mathcal{L}^{\prime}$ be vector bundles over respectively $M$ and $M^{\prime}$. Let $\varphi: M \rightarrow M^{\prime}$. Then a morphism over $\varphi$ is a fibre-preserving fibrewise linear map $\psi: \mathcal{L} \rightarrow \mathcal{L}^{\prime}$. In other words the diagram

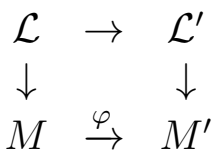

commutes and $\psi: \mathcal{L}_{m} \rightarrow \mathcal{L}_{m}^{\prime}$ is linear. An automorphism is a morphism over the identity which is fibrewise invertible. The group of automorphisms of $\mathcal{L}$ is called the gauge group and denoted $\mathcal{G}(\mathcal{L})$.

A more general definition of vector bundles would allow the vector space $L$ to jump from one connected component of $B$ to the other. We will mostly be interested in connected base spaces and therefore we have no need for such a general definition here. An advantage of our definition is the fact that isomorphism classes of vector bundles in our sense can be described in a uniform way.

\section{A combinatorial interpretation through cocycles}

We need an other definition which allows performing constructions and computations in a combinatorial way.

Definition 3.1.1 let $M$ be a topological space.

1. A cocycle over $M$ with values in the automorphism groups of a vector space $L$ is a pair $(\mathcal{U}, \mathcal{G})$, where $\mathcal{U}$ is an open covering of $M$ and 
$\left\{g_{U V}\right\}_{U, V \in \mathcal{U}}$ is a collection of continuous maps $g_{U V}: U \cap V \rightarrow \mathrm{GL}(L)$ subject to the cocycle condition

$$
g_{U V} g_{V W}=g_{U W} .
$$

2. Two cocycles $(\mathcal{U}, \mathcal{G}),\left(\mathcal{U}, \mathcal{G}^{\prime}\right)$ with the same open covering are called directly equivalent if there exists a family of maps $h_{U}: U \rightarrow \mathrm{GL}(L)$ such that

$$
g_{U V}^{\prime}=h_{U} g_{U V} h_{V}^{-1} .
$$

3. A cocycle $(\mathcal{U}, \mathcal{G})$ is called a refinement of the cocycle $\left(\mathcal{U}_{0}, \mathcal{G}_{0}\right)$ if there exists a map $i: \mathcal{U} \rightarrow \mathcal{U}_{0}$ such that

$$
U \subset i(U)
$$

and for all $U, V$ in $\mathcal{U}$

$$
g_{U V}=\left.g_{i(U) i(V)}\right|_{U \cap V} .
$$

4. Two cocycles will be called equivalent if they have directly equivalent refinements .

We claim that vector bundles over a topological space $M$ up to isomorphism are in one-to-one correspondence with cocycles up to equivalence. Let us explain how the correspondence is obtained: given a cocycle $((\mathcal{U}, \mathcal{G}))$, the cocycle condition ensures that we obtain an equivalence relation $\sim$ on the space

$$
\coprod_{U \in \mathcal{U}} \mathcal{U} \times U \times L
$$

by

$$
(U, x, v) \sim\left(V, x, g_{U V}(x) \cdot v\right) .
$$

Then

$$
\mathcal{L}:=\left(\coprod_{U \in \mathcal{U}} \mathcal{U} \times U \times L\right) / \sim
$$

is the total space of a vector bundle over $M$, where the projection is given by

$$
\pi: \mathcal{L} \rightarrow M, \quad[(U, x, v)] \mapsto x
$$

Conversely, given a vector bundle $(\mathcal{L}, \pi, M)$ we may choose a trivialising covering $\left\{U_{i}\right\}_{i \in I}$ and trivialisations

$$
\varphi_{i}: \pi^{-1}\left(U_{i}\right) \rightarrow U_{i} \times L .
$$


Then we can trivialise the bundle over $U_{i} \cap U_{j}$ in two different ways, namely via

$$
\varphi_{i j}: \pi^{-1}\left(U_{i} \cap U_{j}\right) \hookrightarrow \pi^{-1}\left(U_{i}\right) \stackrel{\varphi_{i}}{\longrightarrow} U_{i} \times L
$$

or

$$
\varphi_{j i}: \pi^{-1}\left(U_{i} \cap U_{j}\right) \hookrightarrow \pi^{-1}\left(U_{j}\right) \stackrel{\varphi_{j}}{\longrightarrow} U_{j} \times L .
$$

Both $\varphi_{i j}$ and $\varphi_{j i}$ are fibre-preserving fibrewise linear homeomorphisms onto $\left(U_{i} \cap U_{j}\right) \times L$ and we can define the cocycle $g_{i j}$ by

$$
\varphi_{j i} \circ \varphi_{i j}^{-1}:\left(U_{i} \cap U_{j}\right) \times L \rightarrow\left(U_{i} \cap U_{j}\right) \times L, \quad(m, v) \mapsto\left(m, g_{i j}(m) v\right) .
$$

Exercise 3.1.2 Show that the constructions described above give indeed rise to a correspondence between isomorphism classes of vector bundles over $M$ and equivalence classes of cocycles.

Example 3.1.3 1. For any topological space $M$, the trivial bundle of rank $\mathrm{n}$ is $M \times \mathbb{R}^{n} \rightarrow M$.

2. Vector bundles may be trivial or non-trivial. The Möbius strip (see Figure 2.1) is a non-trivial line bundle.

3. If $\mathrm{M}$ is a manifold, then the tangent bundle $\mathrm{T} M$ and the cotangent bundle $\mathrm{T}^{*} M$ are vector bundles.

4. As an exercise, the reader is invited to prove that every vector bundle over the interval is trivial.

\subsubsection{Constructions}

\section{Algebraic constructions}

All algebraic operations that can be performed with vector spaces can be performed with vector bundles. As an example, we consider direct sums: let $\mathcal{L}, \mathcal{F}$ be vector bundles over a topological space $M$. Informally speaking, the bundle $\mathcal{L} \oplus \mathcal{F}$ is the bundle with fibre fibre $(\mathcal{L} \oplus \mathcal{F})_{m} \cong \mathcal{L}_{m} \oplus \mathcal{F}_{m}$. We formalise this idea using cocycles: over some open covering $\left\{U_{i}\right\}_{i \in I}$ the bundles are represented by cocycles $g_{i j}$ and $h_{i j}$ respectively. Then we define a bundle $\mathcal{L} \oplus \mathcal{F}$ with via the block-matrix cocycle

$$
g_{i j} \oplus h_{i j}:=\left(\begin{array}{rr}
g_{i j} & 0 \\
0 & h_{i j}
\end{array}\right) .
$$

Similarly, we may construct $\mathcal{L} \otimes \mathcal{F}, \bigwedge^{k} \mathcal{L}, \operatorname{Hom}(\mathcal{L}, \mathcal{F}), \mathcal{L}^{*}$ etc. 


\section{Pullback bundle}

This important construction can be described informally as follows. Let $\pi$ : $\mathcal{L} \rightarrow M$ be a bundle over $M$. Let $f: N \rightarrow M$ be a continuous map where $N$ is any topological space. The pullback bundle $f^{*}(\mathcal{L})$ is the bundle over $M$ whose fibre at $m$ is $\mathcal{L}_{f(m)}$. Again, cocycle makes this construction precise: assume $\mathcal{L} \rightarrow M$ is defined by the cocycle $\left(\left\{U_{i}\right\}_{i \in I},\left\{g_{i j}\right\}_{i, j \in I}\right)$, then the pullback bundlepullback of $\mathcal{L}$ via $f$ is the bundle defined by the cocycle $\left(\left\{f^{-1}\left(U_{i}\right)\right\}_{i \in I},\left\{g_{i j} \circ f\right\}_{i, j \in I}\right)$. alternatively, we can described the total space of the pullback bundle as

$$
f^{*}(\mathcal{L}):=\{(e, n) \in \mathcal{L} \times N \mid \pi(e)=f(n)\}
$$

where the projection is given by the projection to the second factor.

The following fact will be important for us, although we will not prove it here. This is a generalisation of the fact that a vector bundle over the interval is trivial.

Proposition 3.1.4 Suppose that $f_{0}, f_{1}: N \rightarrow M$ are homotopic maps and that $\mathcal{L}$ is a vector bundle over $M$. Then $f_{0}^{*}(\mathcal{L})$ is isomorphic to $f_{1}^{*}(\mathcal{L})$. In particular, any vector bundle over a contractible space is trivial.

When $N$ and $M$ are compact, the proof of this proposition is a fairly straightforward generalisation of the triviality of any vector bundle over the interval.

\section{Sections along a curve}

We will often need to perform operations along a curve in $M$. A convenient way to express such operations is given by the following definition:

Definition 3.1.5 Let $\gamma:[0,1] \rightarrow M$ be a curve and $\mathcal{L} \rightarrow M$ be a vector bundle over $M$. A section along the curve $\gamma$ is a map $v:[0,1] \rightarrow \mathcal{L}$ with $\pi(v(t))=\gamma(t)$. Equivalently, $v \in \Gamma\left(\gamma^{*} \mathcal{L}\right)$.

\subsection{Vector bundles with structures}

So far we have considered cocycles with values in $\operatorname{GL}(L)$, where $L$ is a vector space. These cocycles preserve the linear structure of the vector space $L$. However, we might also want to preserve some additional structure on $L$ like an inner product or a symplectic form or some other tensor. This amounts to restricting the range of our cocycles. We formalise this as follows: 
Let $G$ be a topological group and $\rho: G \rightarrow \operatorname{GL}(L)$ a continuous injective homomorphism - in other words a faithful linear a representation of $G$. We shall in the sequel abusively identify $G$ with its image $\rho(G)$.

Definition 3.2.1 [G-STRUCTURE] $A$ G-atlas of the vector bundle $\mathcal{L}$ over $M$ is an open cover $\left\{U_{i}\right\}$ of $M$ together with a trivialisation of $\left.\mathcal{L}\right|_{U_{i}}$ as $U_{i} \times L$ so that the changes of trivialisations are maps with values in $\rho(G)$. Two $G$-atlases are equivalent if their union is still a $G$-atlas. Finally a $G$-structure is a class of $G$-atlases up to the equivalence above.

Remark 3.2.2 1. In terms of cocycles, we obtain $G$-structures from $G$ valued cocycles which are cocycles $\left(\left\{U_{i}\right\}_{i \in I},\left\{g_{i j}\right\}_{i, j \in I}\right)$, so that $g_{i j}$ are with values in $G$.

2. We also observe that a $G$-structure defines for every $m$ in $M$ a subgroup $G_{m}$ of $\operatorname{GL}\left(\mathcal{L}_{m}\right)$ isomorphic - although not canonically - to $G$. More generally, a $G$-structure defines for every $m$ and $n$ a subset $G_{m, n}$ of $\operatorname{Hom}\left(\mathcal{L}_{m}, \mathcal{L}_{n}\right)$ equipped with a right transitive action of $G_{n}$ and a left transitive action of $G_{n}$, so that $G_{m, p}=G_{m, n} \cdot G_{n, p}$ and $G_{m, m}=G_{m}$.e

We need to extend the previous definitions

Definition 3.2.3 [G-Morphisms] Let $\mathcal{L} \rightarrow M$ and $\mathcal{F} \rightarrow N$ two vectors bundles with $G$-structures.

- A G-morphism is a vector bundle map $\Phi$ from $\mathcal{L}$ to $\mathcal{F}$ over a map $\varphi$ from $M$ to $N$ which is an isomorphism on each fibre and such that the pull back of any $G$-atlas of $\mathcal{F}$ is a $G$-atlas of $\mathcal{L}$.

- A G-isomorphism is a G-morphism over the identity; with the previous notation, this means that $M=N$ and $\varphi=\mathrm{Id}$.

- A G-trivialisation is a G-isomorphism with the trivial G-bundle.

- A G-automorphism is a G-isomorphism of a G-bundle with itself.

Definition 3.2.4 [GAUGe Group] The gauge group $\mathcal{G}(\mathcal{L})$ of the $G$-bundle $\mathcal{L}$ is the group of $G$-automorphisms of the bundle. The gauge group naturally identifies with the group of sections $\sigma: M \rightarrow \operatorname{End}(\mathcal{L})$, so that for every $m$, $\sigma(m)$ belongs to $G_{m}$.

If $\mathcal{L}=M \times L$ is a trivial bundle with the trivial $G$-structure, then $\mathcal{G}(\mathcal{L})=$ $C^{0}(M, G)$. 
Example 3.2.5 Let $B$ be a bilinear form on a vector space $L$. Denote by $\mathrm{SO}(L, B)$ the group of linear automorphisms of $L$ that preserve $B$. Then a $\mathrm{SO}(L, B)$-structure on a vector bundle $\mathcal{L}$ is the same as a continuously varying families of tensors of the same type as $B$ in each fibre. Indeed, given $v, w \in \mathcal{L}_{m}$ we can compute $B(v, w)$ in a trivialisation, and, as the coordinate changes are orthogonal with respect to $B$, this quantity is independent of the trivialisation chosen.

\section{Tensor field}

The following definition and proposition will be used several times in the sequel.

Definition 3.2.6 [TENSOR FIELD] Let $\mathcal{L}_{1}, \ldots, \mathcal{L}_{n}$ and $\mathcal{F}$ be vector bundles over the same space $M$. A map

$$
A: \Gamma\left(\mathcal{L}_{1}\right) \times \ldots \times \Gamma\left(\mathcal{L}_{n}\right) \rightarrow \Gamma(\mathcal{F})
$$

is a tensor field if there exists a section a of $\operatorname{Hom}\left(\mathcal{L}_{1} \otimes \mathcal{L}_{n}, \mathcal{F}\right)$ so that for every point $m$ in $M$

$$
A\left(\sigma^{1}, \ldots, \sigma^{n}\right)_{m}=a_{m}\left(\sigma_{m}^{1}, \ldots, \sigma_{m}^{n}\right) .
$$

Observe that every tensor field $A$ is a morphism of $C^{0}(M)$-algebras, that is for every function $f_{1}, \ldots f_{n}$ on $M$ and sections $\sigma_{i}$ of $\mathcal{L}_{i}$ we have

$$
A\left(f_{1} \sigma_{1}, \ldots, f_{n} \sigma_{n}\right)=f_{1} \ldots f_{n} A\left(\sigma_{1}, \ldots, \sigma_{n}\right) .
$$

Conversely, the following proposition will be helpful:

Proposition 3.2.7 Every morphism of $C^{0}(M)$-algebras

$$
A: \Gamma\left(\mathcal{L}_{1}\right) \times \ldots \times \Gamma\left(\mathcal{L}_{n}\right) \rightarrow \Gamma(\mathcal{F})
$$

is a tensor field.

In the sequel, we shall also abusively use the term tensor field either for the section of $\operatorname{Hom}\left(\mathcal{L}_{1} \otimes \mathcal{L}_{n}, \mathcal{F}\right)$ or the map from $\Gamma\left(\mathcal{L}_{1}\right) \times \ldots \times \Gamma\left(\mathcal{L}_{n}\right)$ to $\Gamma(\mathcal{F})$

Proof: We only sketch the proof. We first observe the following immediate result: if $A$ is a map from $C^{0}(M)$ to itself which is a morphism of $C^{0}(M)$ algebras, then there exists a function $g$ so that $A(f)=g f$. More generally, if $L$ and $V$ are vector spaces and $A$ is a map from $C^{0}(M, L)$ to $C^{0}(M, V)$ which is a morphism of $C^{0}(M)$-algebras, then there exists an element $a$ of $C^{0}(M, \operatorname{Hom}(L, V))$ so that $(A(f))(m)=a(m)(f(m))$. In other words, the proposition above is true for trivial bundles. 
Let us move to the general case using the notation of the proposition. We observe that if $f_{i}, g_{i}$ are sections of $\mathcal{L}_{i}$ equal on some neighbourhood $U$ of $m$ then

$$
A\left(f_{1}, \ldots, f_{n}\right)_{m}=A\left(g_{1}, \ldots, g_{n}\right)_{m}
$$

Indeed, let $\varphi$ be a function equal to 1 at $m$ and with support in $U$, then

$$
A\left(f_{1}, \ldots, f_{n}\right)_{m}=A\left(\varphi \cdot f_{1}, \ldots, \varphi \cdot f_{n}\right)_{m}=A\left(\varphi \cdot g_{1}, \ldots, \varphi \cdot g_{n}\right)_{m}=A\left(g_{1}, \ldots, g_{n}\right)_{m}
$$

After this observation is made, we are reduced to a local situation. Since a vector bundle is locally trivial, a straightforward extension of the initial argument of this proof yields the proposition. Q.E.D.

\subsubsection{Vector bundles over manifolds}

All we have said so far applies to topological spaces, topological groups and continuous maps. The definitions naturally extend to "smooth spaces", "smooth groups" and "smooth maps". By "smooth spaces", we mean manifold; by "smooth group", we mean a Lie group, that is group which is also a manifold in such a way that group multiplication and inversion are smooth maps - as our first (and only examples) we shall only consider classical groups such as $\mathrm{SL}(n, \mathbb{R}), \mathrm{SU}(n)$ etc. A vector bundle is a smooth vector bundle if the defining cocycles can be taken to be smooth. Smooth sections make a natural sense, and we also observe that pullbacks of smooth bundles via smooth maps are smooth. Similarly, algebraic constructions preserves smooth bundles. In the following, we will almost exclusively deal with smooth bundles. Therefore we will make the following conventions:

Convention 3.2.8 All vector bundles, cocycles, $G$-structures, ... are smooth unless otherwise mentioned and we denote by $\Gamma(\mathcal{L})$ the smooth sections of the smooth vector bundle $\mathcal{L}$.

For manifolds there are some bundles which are of particular importance. In the first place these are the tangent bundle $\mathrm{T} M$ and the cotangent bundle $\mathrm{T}^{*} M:=(\mathrm{T} M)^{*}$. Via the algebraic constructions described in Section 3.1.2 we can construct new bundles out of these the bundles. We have encountered the bundles

$$
\bigwedge^{k} \mathrm{~T}^{*} M \quad(k \geq 0)
$$

and using our new notation

$$
\Omega^{k}(M)=\Gamma\left(\bigwedge^{k} \mathrm{~T}^{*} M\right) .
$$


In other words a section of $\bigwedge^{k} \mathrm{~T}^{*} M$ is nothing but a $k$-form on $M$.

More generally, if $\mathcal{L}$ is a vector bundle over $M$, we denote by $\Omega^{k}(M, \mathcal{L})$ the vector space of differential $k$-forms with values in $\mathcal{L}$, that is the space of sections of the bundle $\bigwedge^{k}\left(\mathrm{~T}^{*} M\right) \otimes \mathcal{L}$. By convention, $\bigwedge^{0}\left(\mathrm{~T}^{*} M\right) \otimes \mathcal{L}=\Gamma(\mathcal{L})$.

Finally, we have an easy extension of our previous result about tensor fields:

Proposition 3.2.9 Let $\mathcal{L}_{1}, \ldots, \mathcal{L}_{n}$ and $\mathcal{F}$ be (smooth) vector bundles over the same manifold $M$. Every morphism of $C^{\infty}(M)$-algebras

$$
A: \Gamma\left(\mathcal{L}_{1}\right) \times \ldots \times \Gamma\left(\mathcal{L}_{n}\right) \rightarrow \Gamma(\mathcal{F})
$$

is a (smooth) tensor field.

\subsection{Connections}

Let us start with an informal explanation of what is a connection: if $\pi: \mathcal{L} \rightarrow M$ is a vector bundle and $m, m^{\prime}$ two points in $M$ then the fibres $\mathcal{L}_{m}$ and $\mathcal{L}_{m^{\prime}}$ are isomorphic. However, there is no way in general to construct a natural isomorphism between different fibres for one very good reason: not all vector bundles are trivial.

We can nevertheless remark that any vector bundle over the interval is trivial, an in particular the restriction of the vector bundle to any path joining $m$ to $m^{\prime}$ can be trivialised, albeit non canonically. Informally speaking, a connection is a "coherent" way to trivialise the vector bundle over any path.

Les us be a little more precise in order to justify the next paragraph. A connection will be a procedure with associates to any path $\gamma:[0,1] \rightarrow M$ from $m$ to $m^{\prime}$ an isomorphism

$$
\operatorname{Hol}(\gamma): \mathcal{L}_{m} \rightarrow \mathcal{L}_{m^{\prime}}
$$

called the holonomy of $\gamma$. Such a procedure will be realised by any path in $M$ to a path in $\mathcal{L}$. More precisely, given a curve $\gamma:[0,1] \rightarrow M$ and a starting point $v_{0} \in \mathcal{L}_{\gamma(0)}$ a connection will construct for us a section $v(t)$ along $\gamma$ with $v(0)=v_{0}$. Then the holonomy of $\gamma$ is the map which maps the starting point $v_{0}$ to the endpoint $v(1)$ of $v$. Here is one example: if $M=\mathbb{R}^{n}$ and $\mathcal{L}=\mathrm{TR}^{n}=\mathbb{R}^{n} \times \mathbb{R}^{n}$, the natural lift of $\gamma:[0,1] \rightarrow \mathbb{R}^{n}$ and $(\gamma(0), v) \in \mathcal{L}$ is a constant lift $v(t)=(\gamma(t), v)$. This constant lift is uniquely determined by the property that its derivative in the direction of the path $\gamma$ vanishes. Thus in order to define a connection we need to define what is a "constant section" along a curve; and, in order to define a constant section, we need to define a "derivation" along a curve. This is the idea underlying the next definition. 


\subsubsection{Koszul connection}

Definition 3.3.1 [Koszul Connection] A (Koszul) connection on the vector bundle $\mathcal{L}$ over $M$ is a linear map

$$
\nabla:\left\{\begin{aligned}
\Gamma(\mathcal{L}) & \rightarrow \Omega^{1}(M, \mathcal{L}) \\
\sigma & \mapsto\left(X \mapsto \nabla_{X} \sigma\right)
\end{aligned}\right.
$$

such that for all smooth function $f$ on $M$, we have the Leibniz rule

$$
\nabla_{X}(f \sigma)=\mathrm{d} f(X) \cdot \sigma+f \nabla_{X}
$$

Observe that by definition $\nabla_{f X} \sigma=f \nabla_{X}(\sigma)$.

Alternatively, we can think of a Koszul connection as a bilinear map from $\Gamma(\mathrm{T} M) \times \Gamma(\mathcal{L})$ to $\Gamma(\mathcal{L})$ which associates to a vector field $X$ on $M$ and a section $\sigma$ of $\mathcal{L}$, a new section $\nabla_{X} \sigma$ of $\mathcal{L}$ such that for all function $f$ in $C^{\infty}(M)$, we have

$$
\begin{aligned}
& \nabla_{X} f \sigma=f \nabla_{X}(\sigma)+\mathrm{d} f(X) \cdot \sigma \\
& \nabla_{f X} \sigma=f \nabla_{X}(\sigma) .
\end{aligned}
$$

The relation between the two definitions run as follows: according to the first definition, we may plug a tangent vector $X \in \mathrm{T}_{m} M$ and a section $\sigma$ of $\mathcal{L}$ in a connection in order to obtain an element $\nabla_{X} \sigma$ of $\mathcal{L}_{m}$. But we might as well take a vector field $X \in \Gamma(\mathrm{T} M)$ and a section $\sigma$ of $\mathcal{L}$ in order to produce a section $s$ of $\mathcal{L}$ according to the rule

$$
s_{m}:=\nabla_{X(m)} \sigma .
$$

We will use the abbreviation

$$
\nabla_{X} \sigma:=s
$$

for this construction. Hence, by definition,

$$
\left(\nabla_{X} \sigma\right)(m):=\nabla_{X(m)} \sigma .
$$

The starting example of a Koszul connection is the following one:

Example 3.3.2 Let $\mathcal{L}=M \times L$ be a trivial bundle, where $L$ is a vector space. In particular, $\Gamma(M \times L) \cong C^{\infty}(M, L)$. In this case, we have a preferred connection of $\mathcal{L}$ call the trivial connection and denoted by $\mathrm{D}$. This trivial connection is given by

$$
\mathrm{D}_{X}(\sigma)=\mathrm{D} \sigma(X) .
$$


In particular, we see that every trivial bundle admits a connection. More generally,

Proposition 3.3.3 Every vector bundle admits a connection.

ProOF: If $f_{1}, \ldots, f_{n}$ are functions on $M$ such that $\sum f_{i}=1$ and $\nabla_{i}$ are connections on $M$ then

$$
\nabla=\sum_{i} f_{i} \nabla_{i}
$$

is also a connection. This formula and remarks also makes sense if each $\nabla_{i}$ is only defined on the restriction of the vector bundle over the support of $f_{i}$. Thus, we may take a trivialising cover for $\mathcal{L}$ over $M$, a corresponding smooth partition of unity and combine trivial connections on the trivialising open sets using the partition of unity. Q.E.D.

There will be many connections on a given vector bundle $\mathcal{L}$ and the following proposition shows that the space of connections is an affine space.

Proposition 3.3.4 If $\nabla^{1}, \nabla^{2}$ are connections on $\mathcal{L}$ then there exists a tensor field $A \in \Omega^{1}(M, \operatorname{End}(\mathcal{L}))$ such

$$
\nabla_{X}^{1} \sigma-\nabla_{X}^{2} \sigma=A_{m}(X) \sigma
$$

that for all $m \in M, X \in \mathrm{T}_{m}(M), \sigma \in \Gamma(\mathcal{L})$.

Conversely, given a connection $\nabla$ and a tensor $A \in \Omega^{1}(M, \operatorname{End}(\mathcal{L})), \nabla+A$ is a connection. In particular, the connections on $\mathcal{L}$ form an affine space modelled on $\Omega^{1}(M, \operatorname{End}(\mathcal{L}))$.

This proposition follows from Proposition 3.2.9 and the fact - easily checked from the definition - that

$$
(X, \sigma) \rightarrow \nabla_{X}^{1} \sigma-\nabla_{X}^{2} \sigma
$$

is a morphism of $C^{\infty}(M)$-algebras.

\subsubsection{Constructions}

The constructions (algebraic operations and pullbacks) that we performed above for vector bundles generalise to bundles with connection. We explain some of these constructions here and leave the remaining constructions to the reader. 
1. Suppose $\nabla^{\mathcal{L}}$ and $\nabla^{\mathcal{F}}$ are connections on bundles $\mathcal{L}$ and $\mathcal{F}$ over the same manifold $M$. Then there is a connection $\nabla=\nabla^{\mathcal{L}} \otimes \nabla^{\mathcal{F}}$ on $\mathcal{L} \otimes \mathcal{F}$ which for $X \in \mathrm{T}_{m} M$ and a product section $\sigma=\sigma_{\mathcal{L}} \otimes \sigma_{\mathcal{F}}$ of $\mathcal{L} \otimes \mathcal{F}$ is given by

$$
\nabla_{X} \sigma:=\nabla_{X}^{\mathcal{L}} \sigma_{\mathcal{L}} \otimes \sigma_{\mathcal{F}}(m)+\sigma_{\mathcal{L}}(m) \otimes \nabla_{X}^{\mathcal{F}} \sigma_{\mathcal{F}}
$$

2. If $\nabla$ is a connection on $\mathcal{L}$ then there is a unique connection $\nabla^{*}$ on the dual bundle $\mathcal{L}^{*}$ such that for all $X \in \Gamma(\mathrm{T} M), v \in \Gamma(\mathcal{L})$ and $w \in \Gamma\left(\mathcal{L}^{*}\right)$,

$$
X\langle v \mid w\rangle=\left\langle\nabla_{X} v \mid w\right\rangle+\left\langle v \mid \nabla_{X}^{*} w\right\rangle,
$$

where $\langle\cdot \mid \cdot\rangle: \Gamma(\mathcal{L}) \times \Gamma\left(\mathcal{L}^{*}\right) \rightarrow C^{\infty}(M)$ denotes the dual pairing and $X$ acts as a differential operator on the smooth function $\langle v \mid w\rangle$.

3. If $\nabla$ is a connection on a bundle $\mathcal{L}$ over $M$ and $f: N \rightarrow M$ is a smooth map between manifolds then there is a unique connection, called the pullback connection and denoted $f^{*} \nabla$, on $f^{*} \mathcal{L}$ satisfying

$$
\left(f^{*} \nabla\right)_{X}\left(f^{*} \sigma\right)=f^{*}\left(\nabla_{\mathrm{T} f(X)} \sigma\right),
$$

for all $\sigma \in \Gamma(\mathcal{L})$ and $X \in \mathrm{T} M$.

All these constructions are performed by the following two steps: first considering the situation in the trivial case, then using cocycles. For instance let us explain the construction for the pullback connection. If $\mathcal{L}$ is trivialised over $U$ as $U \times L$, then we can write $\nabla=\mathrm{D}+A$, then we define $f^{*} \nabla$ over $V:=f^{-1}(U)$ as $\mathrm{D}+f^{*} A$. Then, $f^{*} \nabla$ is the unique connection over $V$ satisfying Condition (3.1), thus by uniqueness we see that the definition of $f^{*} \nabla$ coincides over intersection of trivialising open sets - with possibly different trivialisations. It follows that we can define coherently $f^{*} \nabla$ over $M$.

\subsubsection{Gauge equivalence and action of the gauge group}

The definition of pullback enables us to to define an action of the on the space of connections. Indeed, if $f$ is an automorphisms of $\mathcal{L}$ and $\nabla$ is a connection on $\mathcal{L}$, then $f^{*}(\nabla)$ is also a connection on $\mathcal{L}$.

Two connections $\nabla^{1}$ and $\nabla^{2}$ are said to be gauge equivalent if there exists an element $f$ of the gauge group - that is an automorphism of the vector bundle - so that $\nabla^{1}=f^{*}\left(\nabla^{2}\right)$. In other words, gauge equivalence classes are orbits of the gauge group. 


\subsubsection{Holonomy along a path}

We have motivated the introduction of connections by considering the problem of lifting a curve. Now let us see that the formal machinery introduced in the last subsection solves the curve lifting problem:

Definition 3.3.5 [DERIVATION ALONG A CURVE] Let $\mathcal{L}$ be a vector bundle over $M$ and let $\nabla$ be a connection on $\mathcal{L}$. Let $v(t)$ be section along a curve $\gamma:[0,1] \rightarrow M$. Then the derivative of $v(t)$ along $\gamma(t)$ is the section along $\gamma(t)$ given by

$$
\nabla_{\dot{\gamma}} v:=\left(\gamma^{*} \nabla\right)_{\partial_{t}} v
$$

where $\partial_{t}$ is the fundamental vector field of the curve $\gamma$ and $v$ is regarded as a section of $\gamma^{*}(\mathcal{L})$.

Definition 3.3.6 [PARALlEL SECTION ALONG A CURVE] Let $\mathcal{L}$ be a vector bundle over $M$ and let $\nabla$ be a connection on $\mathcal{L}$. A section $v(t)$ along a curve $\gamma:[0,1] \rightarrow M$ is called $\nabla$-parallel along the curve $\gamma$ if

$$
\nabla_{\dot{\gamma}} v=0 \text {. }
$$

Now we can prove that a connection indeed lifts paths:

Proposition 3.3.7 Let $\pi: \mathcal{L} \rightarrow M$ be a vector bundle with connection $\nabla$. Let $\gamma:[0,1] \rightarrow M$ a curve and $v_{0} \in \mathcal{L}_{\gamma(0)}$. Then there exists a unique parallel section $v=v_{v_{0}}$ along $\gamma$ such that $v(0)=v_{0}$. Thus $\nabla$ defines a linear map

$$
\operatorname{Hol}^{\nabla}(\gamma):\left\{\begin{aligned}
\mathcal{L}_{\gamma(0)} & \rightarrow \mathcal{L}_{\gamma(1)} \\
v_{0} & \mapsto v_{v_{0}}(1)
\end{aligned}\right.
$$

Proof: As $[0,1]$ is contractible, the bundle $\gamma^{*} \mathcal{L}$ is trivial. We fix a trivialisation and identify $\gamma^{*} \mathcal{L} \cong[0,1] \times L$. By Proposition 3.3.4 the connection $\gamma^{*} \nabla$ takes the form $\gamma^{*} \nabla=\mathrm{D}+A$ under this identification, where $\mathrm{D}$ denotes the trivial connection and $A$ is some tensor. Then $v \in \Gamma(\mathcal{L})$ is parallel if and only if

$$
\dot{v}=B \cdot v
$$

where $B=-A\left(\partial_{t}\right)$. Thus lifting $\gamma$ with initial point $v_{0}=\left(\gamma(0), \widetilde{v_{0}}\right)$ is the same as solving the ordinary differential equation

$$
\dot{v}=B \cdot v
$$

with initial condition $v(0)=\widetilde{v_{0}}$. The proposition then follows from the existence and uniqueness theorem for ordinary differential equations. Q.E.D. 
Definition 3.3.8 [HolONOMY ALONG A PATH] The map $\mathrm{Hol}^{\nabla}(\gamma)$ from $\mathcal{L}_{\gamma(0)}$ to $\mathcal{L}_{\gamma(1)}$ is called the holonomy of $\nabla$ along $\gamma$.

Example 3.3.9 Let us consider once more the case of the trivial vector bundle $\mathcal{L}=M \times L$ over $M$ with fibre $L$. As usual denote by $\mathrm{D}$ the trivial connection on $\mathcal{L}$. If $\gamma:[0,1] \rightarrow M$ is any path then the constant section $\gamma_{v} \in \Gamma\left(\gamma^{*} \mathcal{L}\right)$ given by $\gamma_{v}(t):=(\gamma(t), v)$ is parallel. Thus $\gamma_{v}$ is the unique parallel lift of $\gamma$ with initial value $v$ and hence $\operatorname{Hol}^{\mathrm{D}}(\gamma)(v)=\gamma_{v}(1)=(\gamma(1), v)$. Thus identifying each fibre $\{x\} \times L$ with $L$ via $(x, v) \rightarrow v$ we see that $\operatorname{Hol}^{\mathrm{D}}(\gamma)$ induces the identity between the two copies of $L$ sitting over $\gamma(0)$ and $\gamma(1)$.

It is easy to see that the connection can be reconstructed from the associated holonomy maps, moreover parallel sections can be described using the holonomy. We leave this as an exercise:

Exercise 3.3.10 Let $\nabla, \nabla^{\prime}$ be connections on a vector bundle $\mathcal{L} \rightarrow M$. Suppose that for all $\gamma:[0,1] \rightarrow M$ the maps $\operatorname{Hol}^{\nabla}(\gamma)$ and $\operatorname{Hol}^{\nabla^{\prime}}(\gamma)$ coincide. Show that $\nabla=\nabla^{\prime}$.

Exercise 3.3.11 Let $\sigma$ be a section of $\mathcal{L}$. Then $\sigma$ is parallel if and only if for every path $\gamma$ from $x$ to $y$, we have $\sigma(y)=\operatorname{Hol}_{\nabla}(\gamma) \cdot \sigma(x)$.

\subsubsection{Holonomy and linear differential equations}

Parallel section and holonomies have interpretations using differential equations. We summarise this in the following Proposition whose proof is left to the reader.

Proposition 3.3.12 Let $\gamma:[a, b] \rightarrow M$ be a path. We choose a trivialisation of $\mathcal{L}$ along $\gamma$ as $[a, b] \times \mathcal{L}_{0}$ so that $\nabla=\mathrm{D}+A$ where $\mathrm{D}$ is the trivial connection and $A \in \Omega^{1}\left([a, b]\right.$, $\left.\operatorname{End}\left(\mathcal{L}_{0}\right)\right)$. Let $B=A\left(\partial_{t}\right)$. A section $\sigma$ along $\gamma$ is now interpreted as a map $X:[a, b] \rightarrow \mathcal{L}_{0}$. Then $\sigma$ is parallel along $\gamma$ if and only if $X$ satisfies

$$
(*) \frac{\mathrm{d}}{\mathrm{d} t} X(t)=B(t) X(t) .
$$

In particular the holonomy from $\gamma(u)$ to $\gamma(s)$ along $\gamma$ is the resolvent of this equation.

\subsubsection{Curvature}

One should notice that a connection does not produce just one canonical isomorphism between different fibres, but rather one isomorphism for each curve 
$\gamma$ in $M$ joining the two base points. Different curves may lead to different holonomy maps. We, however, will mainly be interested in connections whose holonomy depends only on the homotopy class (relative endpoints) of a curve. In order to characterise these connections we introduce the following notion:

Definition 3.3.13 Let $\nabla$ be a connection on a vector bundle $\pi: \mathcal{L} \rightarrow M$. Then the curvature of $\nabla$ is

$$
R^{\nabla}: \mathrm{T} M \times \mathrm{T} M \rightarrow \operatorname{End}(\mathcal{L})
$$

which is given by

$$
R^{\nabla}(X, Y) \sigma:=\nabla_{X} \nabla_{Y} \sigma-\nabla_{Y} \nabla_{X} \sigma-\nabla_{[X, Y]} \sigma,
$$

for vector fields $X, Y \in \Gamma(\mathrm{T} M)$ and a section $\sigma \in \Gamma(\mathcal{L})$.

The connection is called flat if and only if $R^{\nabla}$ vanishes identically.

Exercise 3.3.14 Show that $R^{\nabla}$ defines a tensor field $R^{\nabla} \in \Omega^{2}(M, \operatorname{End}(\mathcal{L}))$.

Example 3.3.15 (i) If D is the trivial connection of $\mathcal{L}=M \times L$ then $R^{\mathrm{D}}=0$ by Schwarz lemma and thus $\mathrm{D}$ is flat.

(ii) Suppose $\mathcal{L}=M \times \mathbb{R}$ is the trivial line bundle over $M$. Then any connection on $\mathcal{L}$ is of the form $\nabla^{\alpha}=\mathrm{D}+\alpha$, where $\alpha$ is a 1 -form on $M$ and

$$
R^{\nabla^{\alpha}}=\mathrm{d} \alpha
$$

Thus $\mathrm{D}+\alpha$ is flat if and only if $\alpha$ is closed.

\subsubsection{Flat connections}

The curvature will measure whether the holonomy is invariant under homotopies. The next theorem is fundamental and we shall only prove the easy part of it (see comments section for textbook references).

Theorem 3.3.16 Let $\nabla$ be a connection on a vector bundle $\pi: \mathcal{L} \rightarrow M$. Then the following are equivalent:

(i) $\nabla$ is flat, in other words $R^{\nabla} \equiv 0$.

(ii) If $\gamma, \gamma^{\prime}:[0,1] \rightarrow M$ are homotopic with fixed extremities then

$$
\operatorname{Hol}^{\nabla}(\gamma)=\operatorname{Hol}^{\nabla}\left(\gamma^{\prime}\right)
$$


(iii) The bundle $\mathcal{L}$ admits a trivialisation over every simply-connected open subset of $M$ in which $\nabla$ is trivial.

Notice in contrast to (iii) that not every bundle on a simply connected space is trivial: the famous case is the 2-sphere and its tangent space; one can not comb a sphere [Mil97].

Proof: The obvious implication is (iii) $\Rightarrow(\mathrm{i})$, which follows from the fact that the trivial connection is flat. Let us show that (ii) implies (iii): given a simplyconnected open subset $U$ of $M$ fix a point $x_{0} \in U$ and join any point $x \in U$ with $x_{0}$ via a path $\gamma_{x}$ contained in $U$. Then the map $\operatorname{Hol}(x):=\operatorname{Hol}^{\nabla}\left(\gamma_{x}\right): \mathcal{L}_{x} \rightarrow \mathcal{L}_{x_{0}}$ depends only on $x$ but not on the choice of $\gamma_{x}$ because any two paths in $U$ joining $x$ and $x_{0}$ are homotopic relative endpoints (even in $U$ ). Then the map

$$
i:\left.\mathcal{L}\right|_{U} \rightarrow U \times \mathcal{L}_{x_{0}}, \quad v \mapsto(\pi(v), \operatorname{Hol}(\pi(v))(v)
$$

is a trivialisation with trivial holonomy and thus $\nabla$ is trivial on $U$ by Exercise 3.3.10. Finally, the hard part of the proof is to show that (i) $\Rightarrow$ (ii). For this we refer to the literature quoted in the Section 3.6. Q.E.D.

Note that there is also an easy direct argument for the implication (iii) $\Rightarrow$ (ii). Namely, the holonomy of the trivial connection does not depend on a chosen path. A special case of the last theorem is given by the following corollary:

Corollary 3.3.17 Let $\nabla$ be a flat connection on a vector bundle $\pi: \mathcal{L} \rightarrow M$ and $x_{0} \in M$. Then, the map

$$
\pi_{1}\left(M, x_{0}\right) \rightarrow \operatorname{GL}\left(\mathcal{L}_{x_{0}}\right), \quad[\gamma] \mapsto \operatorname{Hol}^{\nabla}(\gamma)
$$

is a group homomorphism called the holonomy homomorphism of $\nabla$.

\subsubsection{Connections preserving structures}

What is a connection in the world of $G$-structures? When the bundle is equipped with a $G$-structure, it makes sense to speak of a connection preserving the $G$-structure. Let $G$ be a subgroup of $\operatorname{GL}(L)$ which is a smooth submanifold. Recall the Lie algebra $\mathfrak{g}$ of $G$ is the tangent space to $G$ at the origin which we consider as a vector subspace of $\operatorname{End}(L)$.

Definition 3.3.18 Let $M$ be a manifold and $\mathcal{L} \rightarrow M$ be a vector bundle equipped with a $G$-structure. A connection $\nabla$ on $\mathcal{L}$ is a $G$-connection if, for 
any open set $U$, for any $G$-trivialisation over $U$, there exists a 1-form $\omega$ on $U$ with values in $\mathfrak{g}$ such that in that trivialisation

$$
\left.\nabla\right|_{U}=\mathrm{D}+\omega
$$

where $\mathrm{D}$ is the trivial connection.

Example 3.3.19 Suppose $G=\mathrm{SO}(B)$. Then a $G$-structure is the same as a fibrewise tensor of the same type as $B$, and a $G$-connection is a connection for which this family of tensors is parallel.

The following is a straight-forward verification:

Proposition 3.3.20 Let $\nabla$ be a $G$-connection on a vector bundle $\pi: \mathcal{L} \rightarrow M$ and $x_{0} \in M$.

(i) Then the curvature of $\nabla$ is given by

$$
R^{\nabla}=\Omega,
$$

where 2 -form $\Omega \in \Omega^{2}(M, \mathfrak{g})$.

(ii) If $\nabla$ is flat then the holonomy homomorphism is with values in $G_{x_{0}}$,

(iii) Finally, if two connections are gauge equivalent, then the associated monodromy homomorphisms are conjugated by an element of $G_{x_{0}}$.

\subsubsection{The holonomy map of a flat connection}

The previous proposition associates to every flat connection a conjugacy class of homomorphism in $G$ since $G_{x_{0}}$ is isomorphic up to conjugation with $G$. This leads to the following definition.

Definition 3.3.21 [HOLONOMY REPRESENTATION OF A FLAT CONNECTION] Let $\nabla$ be a flat $G$-connection. Then the holonomy representation of $\nabla$ is the conjugacy class of the representation of $\pi_{1}(M)$ in $G$ described in Proposition 3.3.20.

The following proposition is crucial.

Proposition 3.3.22 Two gauge equivalent flat connections yields conjugate representations. Conversely, if two flat connections have the same holonomy they are conjugate. 
Proof: The first part of the proposition is obvious using the last statement in Proposition 3.3.20. Let us concentrate on the second part. We will produce effectively the gauge equivalence: let $\nabla^{1}$ and $\nabla^{2}$ be two connections with the same holonomy representation. Let us fix a base point $x_{0}$. Then we choose for every $x$ in $M$ a path $c_{x}$ from $x_{0}$ to $x$. We finally define

$$
g_{x}=\left(\operatorname{Hol}^{\nabla^{1}}\left(c_{x}\right)\right)^{-1} \operatorname{Hol}^{\nabla^{2}}\left(c_{x}\right) .
$$

Since $\nabla^{1}$ and $\nabla^{2}$ are flat, $g_{x}$ only depends on the homotopy class of $c_{x}$. Since moreover the two connections have the same holonomy, the reader is required to check tha $g_{x}$ is independent on the choice of the homotopy class. It follows that for every path joining $\gamma$ two points $x$ and $y$,

$$
g_{y}^{-1} \mathrm{Hol}^{\nabla^{1}}(\gamma) g_{x}=\mathrm{Hol}^{\nabla^{2}}(\gamma)
$$

Let finally $g: x \rightarrow g_{x}$ be the associated element of the gauge group. Then, the previous equation says, the holonomy of every path is the same for $g^{*} \nabla_{1}$ and $\nabla^{2}$. Then by Exercise 3.3.10 $g^{*} \nabla_{1}=\nabla^{2}$ and thus $\nabla_{1}$ and $\nabla_{2}$ are gauge equivalent. Q.E.D.

\subsection{Combinatorial versions of connections}

We define in this section two combinatorial analogues of connections and flat connections together with the associated gauge equivalences. The two next paragraphs mirror the combinatorial constructions of the fundamental group in Section 2.4. In the next section, we will show all these notions are equivalent.

\subsubsection{Discrete connections on ribbon graphs}

We now explain what is a connection in the world of ribbon graphs. Let $\Gamma$ be a ribbon graph whose set of edges is $E$, set of vertices is $V$ and set of faces is $F$.

Definition 3.4.1 [Discrete $G$ - COnnections] $A$ discrete $G$-connection on $\Gamma$ is a map

$$
\nabla: E \rightarrow G, \quad e \mapsto g_{e},
$$

satisfying $g_{\bar{e}}=g_{e}^{-1}$. We will write $\nabla=\left\{g_{e}\right\}_{e \in E}$.

Definition 3.4.2 [Discrete Gauge equivalence] Denote by $\left(G^{E}\right)^{\prime} \subset G^{E}$ the set of discrete connections. The group $G^{V}$ is called the gauge group of $\Gamma$ The gauge group acts on the space of connections via

$$
\left\{g_{v}\right\}_{v \in V} \cdot\left\{h_{e}\right\}_{e \in E}:=\left\{g_{e_{+}} h_{e} g_{e_{-}}\right\}_{e \in E} .
$$

Two discrete connections in the same $G^{V}$-orbit are called gauge equivalent. 
Recall that a sequence of edges $\left(e_{1}, \ldots, e_{n}\right)$ is a path on $\Gamma$ if $\left(e_{j}\right)_{+}=\left(e_{j+1}\right)_{-}$ for $j=1, \ldots, n-1$. Denote by $\mathcal{P}$ the space of paths in $\Gamma$.

Definition 3.4.3 [PATHS AND HOLONOMY] Let $\nabla=\left\{g_{e}\right\}_{e \in E}$ be a discrete connection. The holonomy of $\nabla$ is the map

$$
\operatorname{Hol}(\nabla): \mathcal{P} \rightarrow G, \quad\left(e_{1}, \ldots, e_{n}\right) \mapsto g_{e_{1}} \cdots g_{e_{n}} .
$$

Faces are special paths:

Definition 3.4.4 [Flat COnneCtions] The curvature of $\nabla$ is the map

$$
R^{\nabla}:=\{\operatorname{Hol}(f)\}_{f \in F} \in G^{F} .
$$

$A$ discrete connection is called flat if $R^{\nabla}=\mathrm{Id}$.

We then have the discrete analogue of Theorem 3.3.16

Proposition 3.4.5 Let $\nabla$ be a discrete flat connection on a ribbon graph. Let $e$ and $f$ be two homotopic path with the same endpoints. then

$$
\operatorname{Hol}^{\nabla}(e)=\operatorname{Hol}^{\nabla}(f) .
$$

The proof of the proposition relies on the following observation: homotopically trivial loops have trivial holonomy. Let $S$ be the surface associated to a ribbon graph. The previous proposition and the description of $\pi_{1}(S)$ using ribbon graphs 2.4.3 yields the following definition.

Definition 3.4.6 [HolONOMY REPRESENTATION] Let $\Gamma$ be a ribbon graph filling a surface $S$. The holonomy map gives rises to the holonomy representation of the discrete $G$-connection from $\pi_{1}(S)$ to $G$, which associates to every discrete closed path its holonomy.

REMARK: Two gauge equivalent discrete connections give rise to conjugate holonomy representations.

\subsubsection{Local systems}

We now define discrete $G$-connections in the context of covers of surfaces in the same spirit that we defined fundamental groups using covers of a surface.

If $\mathcal{U}=\left\{U_{i}\right\}_{i \in I}$ is a covering, let

$$
I^{(n)}:=\left\{\left(i_{1}, \ldots, i_{n}\right) \in I^{n} \mid g_{i_{1}} \cap \ldots g_{i_{n}} \neq \emptyset\right\} .
$$


Definition 3.4.7 [G-LOCAL SYSTEMS] Let $M$ be a manifold and let $\mathcal{U}=$ $\left\{U_{i}\right\}_{i \in I}$ be a covering. Given a group $G$, a $G$-local system over $M$ on $\mathcal{U}$ is a collection of elements $\left(g_{i j}\right)_{(i, j) \in I^{(2)}}$ of elements of $G$ such that moreover

$$
g_{i j} g_{j k}=g_{i k},
$$

whenever $(i, j, k) \in I^{(3)}$. Observe that every local system on $\mathcal{U}$ gives rise to a local system on any refinement of $\mathcal{U}$.

The gauge group of the covering $\mathcal{U}$ is the group $G^{I}$. The gauge group acts on the space of local systems over $U$ by

$$
\left(h_{i}\right)_{i \in I}:\left(g_{i j}\right) \mapsto h_{i} g_{i j} h_{j}^{-1} .
$$

Two $G$-local systems on the same covering $\mathcal{U}$ are called gauge equivalent as local systems if they lie in the same orbit of the gauge group.

Two G-local systems are equivalent as local systems if they are in they are gauge equivalent on some common refinement.

REMARK: If $G$ is abelian, then in the language of Cech cohomology a $G$-local system is just a Čech-1-cocycle and an equivalence class of such systems is just the corresponding cohomology class. Then standard arguments in cohomology show that the set of equivalence classes of $G$-local systems are naturally identified with $H^{1}(M, G)$. Therefore one should think of the collection of equivalence classes of $G$-local systems as a non-abelian version of $H^{1}(M, G)$.

We defined in Section 2.4.2 fundamental groups using covers. We extend our previous construction now:

Definition 3.4.8 [Holonomy of A G-LOCAL SYSTEM] The holonomy of the path $\left(i_{0}, \ldots, i_{p}\right)$ for the $G$-local system $\left(g_{i j}\right)$ is the element $g_{i_{0}, i_{1}} \ldots g_{i_{n-1}, i_{n}}$ of $G$. By definition of a local system, the holonomy of a trivial loop is trivial. The holonomy representation is the map from $\pi_{1}^{\mathcal{U}}(M)$ to $G$ which associates to each path its holonomy.

One more time two gauge equivalent local systems yield conjugate representations, we leave this check to the reader.

\subsection{Four models of the representation variety}

In this paragraph, we gather the information that we have collected on smooth and discrete connections, local systems and representations of the fundamental group of the surface. All these objects will be identified in this section up to some equivalence. Let us be more precise: we have four collections of objects to which we associated "holonomy" representations, namely 
(1) Pairs $(\mathcal{L}, \nabla)$, where $\mathcal{L}$ is a $G$-vector bundle over the surface $S$ and $\nabla$ is a flat $G$-connection on $\mathcal{L}$, up to gauge equivalence.

(2) Equivalence classes of discrete flat $G$-connections with respect to a filling ribbon graph $\Gamma$, again up to gauge equivalence.

(3) Equivalence classes of $G$-local systems on $S$ on a given covering.

(4) Homomorphisms of $\pi_{1}(S)$ into $G$ up to conjugation.

These four collections of objects give rise to different models for the representation variety, each of which will turn out to be useful at some stage. The equivalence of the descriptions (1), (3) and (4) holds for general manifolds and we shall prove them in this generality.

Remark again that when $G=\mathbb{R}$, the above equivalences are the well-known isomorphisms between de Rham, simplicial and Čech cohomology as well as Hurewicz Theorem

We shall denote by $\mathcal{M}_{i}$ with $i \in\{1,2,3,4\}$ the corresponding set of equivalence classes in the four cases under consideration.

We shall also denote by $\operatorname{Rep}\left(\pi_{1}(S), G\right)=\mathcal{M}_{4}$ the space $\operatorname{Hom}\left(\pi_{1}(S), G\right) / G$ where $G$ acts on $\operatorname{Hom}\left(\pi_{1}(S), G\right)$ by conjugation.

\subsubsection{Flat connections and local systems}

We need a definition.

Definition 3.5.1 [GoOd COVERING] An open covering $\left\{U_{i}\right\}_{i \in I}$ of a manifold $M$ is called a good covering if it satisfies the following condition. For all $n \in \mathbb{N}$ and all $i_{1}, \ldots, i_{n} \in I$ the subset

$$
U_{i_{1}, \ldots, i_{n}}:=U_{i_{1}} \cap \cdots \cap U_{i_{n}}
$$

is empty or contractible.

Any manifold admits a good covering, but we will not prove this here since this is more easily achieved using riemannian geometry. Anyhow, we are mainly interested in the case of surfaces and in this case one can construct good coverings associated to ribbon graphs.

Theorem 3.5.2 Let $M$ be a manifold. Then there exist a one-to-one correspondence preserving the holonomy between gauge equivalence classes of $G$-local systems on a good covering and flat $G$-connections. 
Proof: Let $\left(g_{i j}\right)$ be a $G$-local system for the good covering $\left\{U_{i}\right\}_{i \in I}$ of $M$. We view $g_{i j}$ as a constant map $U_{i} \cap U_{j} \rightarrow G \subset \operatorname{End}(L)$. Then $g_{i j}$ defines a cocycle hence a vector bundle over $M$, which we call $\mathcal{L}$. By construction we have trivialisations $\left.\mathcal{L}\right|_{U_{i}} \cong U_{i} \times L$ such that the transition maps are the constant functions $g_{i j}$. On $U_{i} \times V$ we have the trivial connection $D_{i}$ and thus we obtain a connection $\nabla_{i}$ on $\left.\mathcal{L}\right|_{U_{i}}$ via pullback. Since the $g_{i j}$ are constant linear maps and the derivative of a constant map with respect to the trivial connection is the linear map itself we see that $\nabla_{i}$ and $\nabla_{j}$ coincide on $\left.\mathcal{L}\right|_{U_{i, j}}$. Thus there exists a unique connection $\nabla$ on $\mathcal{L}$ such that $\left.\nabla\right|_{U_{i}}=\nabla_{i}$. Thus we have produced a bundle $\mathcal{L}$ with connection $\nabla$ from a $G$-local system. Conversely, given a bundle with connection we conclude from Theorem 3.3.16 that it trivialises over each of the sets $U_{i}$ and that the connection is the pullback of a trivial connection. Then the coordinate changes preserve the connection and thus are locally constant, hence constant. It is easy to check that this gives rise to the correspondence claimed in the proposition. Q.E.D.

\subsubsection{Local systems and representations}

Theorem 3.5.3 Let $M$ be a manifold and $G$ be a Lie group. Then the holonomy map from the space of flat G-local systems on a good covering - up to gauge equivalence - to $\operatorname{Rep}\left(\pi_{1}(M), G\right)$ is a bijection.

Equivalently, using the previous correspondence

Theorem 3.5.4 Let $M$ be a manifold and $G$ be a Lie group. Then the holonomy map from the space of flat $G$-connections up to gauge equivalence to $\operatorname{Rep}\left(\pi_{1}(M), G\right)$ is a bijection.

If $G$ is abelian then this correspondence identifies the first cohomology group $H^{1}(M, G)$ with $\operatorname{Hom}\left(\pi_{1}(M), G\right)$. In this case, the correspondence is just the Hurewicz Theorem 2.5.9.

Proof: Let us first remark that the universal covering $\tilde{M} \rightarrow M$ defines a $\pi_{1}(M)$ local system.

Indeed, let $\mathcal{U}=\left\{U_{i}\right\}_{i \in I}$ be a good covering. Observe that if $\pi: \tilde{M} \rightarrow M$ is the universal cover, then for very $i$ in $I$, we have

$$
\pi^{-1}\left(U_{i}\right)=\sqcup_{g \in \pi_{1}(M)} g V_{i},
$$

Then, every path in the covering $\left\{U_{i}\right\}_{i \in I}$ can be lifted as a path in the covering $\left\{g . V_{i}\right\}_{g \in \Lambda, i \in I}$. We now choose for every $i$ in $I$ a path $p_{i}$ from $i_{0}$ to $i$ in $\left\{U_{i}\right\}_{i \in I}$. Then if $U_{i} \cap U_{j} \neq \emptyset$ the loop $p_{i}, p_{j}^{-1}$ lifts to an element $\lambda_{i, j}$ in $\Lambda$. Then, one checks that $\lambda_{i, j}$ is indeed a local system. 
Therefore, if $\psi$ is a representation of $\pi_{1}(M)$ into $G,\left\{\lambda_{i, j}\right\}$ defines a $G$-local system whose holonomy is precisely $\psi$. We have just proved that the holonomy map from $G$-local systems - on $\mathcal{U}$ - is surjective.

Using the previous correspondence and Proposition 3.3.22, we see that this map is also injective. Q.E.D.

\subsubsection{Smooth and discrete connections}

We have now established the correspondence $(1) \leftrightarrow(3) \leftrightarrow$ (4) for general manifolds $M$. In the case of surfaces we can also give another equivalent description (2) using ribbon graphs. Thus let $S$ be a compact oriented surface and let $\Gamma$ be a filling ribbon graph for $S$.

Theorem 3.5.5 Let $S$ be a surface and $\Gamma$ a filling ribbon graph for $S$. Then there exists a holonomy preserving one to one correspondence between flat connections up to gauge equivalence on $S$ and flat discrete connections on $\Gamma$ up to gauge equivalence.

Proof: Let $E$ be a $G$-vector bundle on $S$. We assume $\Gamma$ is embedded in $S$. We choose a $G$-trivialisation in the neighbourhood of every vertex of the graph. Then the holonomy of every edge is well defined as an element of $G$. Therefore we have associated to a flat connection a discrete connection on the ribbon graph with the same holonomy.

In order to complete the proof, by Theorem 3.5.4, it remains to prove that every discrete connection can be obtained this way. For that we first consider a subdivision $\Gamma_{0}$ of $\Gamma$ so that all edges of $\Gamma_{0}$ connect different vertices and all faces are triangles.

This can be achieved by adding a vertex in the middle of every edge, and face and connect the created vertex in each face to all the vertex in the boundary of the face.

Then, we can construct a good covering $\left\{U_{v}\right\}_{v \in V}$ so that

- $U_{v}$ is a contractible neighbourhood of the vertex $v$.

- $U_{v}$ intersects $U_{w}$ if and only if $v$ and $w$ are connected by an edge $e$. Moreover $e$ intersects $U_{v} \cap U_{w}$.

- Finally $U_{v} \cap U_{w} \cap U_{z} \neq \emptyset$ if and only if the vertices $v, w$ and $w$ are the three vertices of a common face $f$.

We can associate to a discrete connection $g$ on $\Gamma_{0}$ the $G$-local system such that $g_{v, w}$ is the the value $g(e)$ of the connection on the edge from $v$ to $w$. By 
construction, the smooth connection associated to this local system is such that the holonomy of $e$ is $g(e)$. It follows that every discrete connection on $\Gamma_{0}$ can be realised as coming from a smooth connection.

We finally choose an injective map $I$ from the set $E$ of vertices of $\Gamma$ to the set $E_{0}$ of vertices in $\Gamma_{0}$, so that $I(e)$ is a subset of $e$. This defines a map $I^{*}$ from the set of discrete flat connections on $\Gamma$ to the set of discrete flat connection $\Gamma_{0}$ by the following rule: $I^{*} g(e)=g(e)$ if $e$ belongs to $I(E)$ and the identity otherwise. By construction, if $\nabla$ is a smooth connection such that its associated discrete connection on $\Gamma_{0}$ is $I^{*}(g)$, then its associated connection on $\Gamma$ is $g$. Q.E.D.

\subsection{Comments, references and further read- ings}

The material presented about vector bundles and connections from the differentiable viewpoint is classical. Usual references - with complete proofs - are Milnor-Stasheff "Characteristic classes" [MS74] Kobayashi-Nomizu "Foundations of differential geometry. Vol. I" [KN96] and Spivak very complete "comprehensive introduction to differential geometry" [Spi79a, Spi79b]. A more modern treatment is [Lee09].

The notion of a discrete connection is transparent in lattice models of gauge theory. In particular the fat graph description is found in Fock-Rosly's articles [FR97, FR99]. 


\section{Chapter 4}

\section{Twisted Cohomology}

In this chapter we study and generalise cohomology in the presence of a flat vector bundle. We justify the construction by the need to study the infinitesimal linear structure of the representation variety. Similarly to our discussion of cohomology in the first chapter and following the philosophy of these lecture notes series we use different languages to describe twisted cohomology: differential and combinatorial. We stay elementary and describe the isomorphisms between the different construction in a pedestrian way.

In the last section we discuss the torsion of a flat vector bundle. Our aim is, following Witten, to relate the volume associated to Poincaré duality to this invariant.

\subsection{De Rham version of twisted cohomology}

\subsubsection{Motivation: variation of connection}

Let us return to our de Rham model of the representation variety, namely the space of flat $G$-connections modulo gauge equivalence. Let $[\nabla]$ be an equivalence class in this model. The question that motivates for us the introduction of twisted cohomology is the following one: How should a candidate for the tangent space of the representation variety at $[\nabla]$ look like? The tangent space of the space of flat $G$-connections at $\nabla$ should of course be something like the space of all possible variations of $\nabla$ preserving the flatness condition. On the other hand, as we are dealing with equivalence classes, the tangent space at the class $[\nabla]$ should be a quotient of this space of variations by the subspace of those variations which come from a gauge transformation.

Let us try to make these ideas precise.

What does it mean to vary $\nabla$ amongst flat connections? Recall from Propo- 
sition 3.3.4 that any connection $\nabla^{\prime}$ on the same bundle can be written as $\nabla^{\prime}=\nabla+A$, where $A \in \Omega^{1}(M, \operatorname{End}(\mathcal{L}))$. Thus varying $\nabla$ inside the space of all connections means to choose a smooth family of elements $A_{t}$ in the vector space $\Omega^{1}(M, \operatorname{End}(\mathcal{L}))$ and to define $\nabla_{t}:=\nabla+A_{t}$. An infinitesimal variation is then given by $\dot{\nabla}:=\left.\frac{\mathrm{d}}{\mathrm{d} t}\right|_{t=0} A_{t}$. However, we want to vary $\nabla$ only in the subspace of flat connections. Thus we have to single out those infinitesimal variations coming form families $\nabla_{t}$ for which the curvature is zero. Let us call these infinitesimal variations flat (infinitesimal) variations. Let us define the infinitesimal change of curvature by $\dot{R}:=\left.\frac{\mathrm{d}}{\mathrm{d} t}\right|_{t=0} R^{\nabla_{t}}$. Then we want to express $\dot{R}$ in terms of $\dot{\nabla}$. This is indeed possible. We first introduce notation. On the endomorphism bundle $\operatorname{End}(\mathcal{L})$ there is a connection $\nabla^{\operatorname{End}(\mathcal{L})}$ induced from $\nabla$ and given by

$$
\left(\nabla_{X}^{\operatorname{End}(\mathcal{L})} A\right)(u):=\nabla_{X}(A u)-A\left(\nabla_{X} u\right)
$$

where $A$ is a section of $\operatorname{End}(\mathcal{L})$ and $u$ is a section of $\mathcal{L}$. We usually will abusively denote this connection by $\nabla$. Let

$$
\mathrm{d}_{\nabla}^{1}: \Omega^{1}(M, \operatorname{End}(\mathcal{L})) \rightarrow \Omega^{2}(M, \operatorname{End}(\mathcal{L}))
$$

be defined by

$$
\mathrm{d}_{\nabla}^{1}(\alpha)(X, Y):=\nabla_{X}(\alpha(Y))-\nabla_{Y}(\alpha(X))-\alpha([X, Y]),
$$

where $\alpha$ belongs to $\Omega^{1}(M, \operatorname{End}(\mathcal{L}))$ and $X, Y$ are tangent vector fields on $M$.

Proposition 4.1.1 The infinitesimal variation of curvature is given by

$$
\dot{R}=\mathrm{d}_{\nabla}^{1} \dot{\nabla}
$$

Proof: This follows from the fact that

$$
R^{\nabla_{t}}=R^{\nabla}+\mathrm{d}_{\nabla}^{1} A_{t}+\frac{1}{2}[A, A]
$$

where the commutator is defined by

$$
[A, B](X, Y):=[A(X), B(Y)]-[A(Y), B(X)] .
$$

Q.E.D.

As a consequence, an infinitesimal variation $\dot{\nabla}$ is flat if and only if

$$
\mathrm{d}_{\nabla}^{1} \dot{\nabla}=0
$$


Now let us study those variations which come from a gauge transformation . These should be regarded as trivial and our space of cocycles should be divided by the space of these variations in order to obtain the tangent space of $[\nabla]$ (rather then that of $\nabla$ ). To describe the trivial variations, let $g_{t}$ be a smooth path with values in the gauge group with $g_{0}=i d$. Then a trivial variation is one of the form

$$
\nabla_{t}=g_{t}^{-1} \nabla g_{t}
$$

We need to understand the corresponding infinitesimal variation. For this we need some more machinery. Let $\mathcal{L}$ denote the vector bundle on which $\nabla_{0}$ lives. This is represented by a $G$-valued cocycle. If we replace the action of $G$ on the typical fibre $V$ of $\mathcal{L}$ by the adjoint action of $G$ on its Lie algebra $\mathfrak{g}$, then this cocycle defines for us a bundle $\mathfrak{g}_{\mathcal{L}}$ with typical fibre $\mathfrak{g}$ over $M$ called the associated Lie algebra bundle. Moreover there is a canonical inclusion of $\mathfrak{g}_{\mathcal{L}}$ in $\operatorname{End}(\mathcal{L})$. The above variation then defines a section $\dot{g}$ of $\mathfrak{g}_{\mathcal{L}}$ by

$$
\dot{g}_{m}:=\left.\frac{\mathrm{d}}{\mathrm{d} t}\right|_{t=0} g_{t} .
$$

As $\nabla$ is a $G$-connection the connection $\nabla^{\operatorname{End}(\mathcal{L})}$ restricts to a connection on $\mathfrak{g}_{\mathcal{L}}$. Now we can finally describe the infinitesimal variation associated with $\nabla_{t}$ as above:

Proposition 4.1.2 Let notation be as above. Then $\dot{\nabla}=\nabla^{\operatorname{End}(\mathcal{L})} \dot{g}$.

Proof: As both sides of the equation are defined in local terms we can work in a trivialisation. Thus fix an open subset $U$ of $M$ such that $\left.\mathcal{L}\right|_{U} \cong U \times L$. Under this trivialisation $\nabla$ becomes identified with a connection of the form $\mathrm{D}+A$, where $\mathrm{D}$ denotes as usual the trivial connection. Identifying $\left.\operatorname{End}(\mathcal{L})\right|_{U} \cong$ $U \times \operatorname{End}(V)$ the induced connection $\nabla^{\operatorname{End}(\mathcal{L})}$ then becomes identified with $\mathrm{D}+$ $\operatorname{ad}(A)$, where $\operatorname{ad}(A) \in \Omega^{1}(U) \otimes \operatorname{End}(\operatorname{End}(\mathcal{L}))$ is given by

$$
(\operatorname{ad}(A)(X))(B):=[A(X), B] .
$$

Now,

$$
\begin{aligned}
\left(g_{t}^{-1} \nabla g_{t}\right)(u) & =\left(g_{t}^{-1}(\mathrm{D}+A) g_{t}\right)(u) \\
& =g_{t}^{-1} \mathrm{D}\left(g_{t} u\right)+\left(g_{t}^{-1} A g_{t}\right)(u) \\
& =g_{t}^{-1} g_{t}(\mathrm{D} u)+g_{t}^{-1}\left(\mathrm{D} g_{t}\right)(u)+\left(g_{t}^{-1} A g_{t}\right)(u) \\
& =(\mathrm{D} u)+g_{t}^{-1}\left(\mathrm{D} g_{t}\right)(u)+\left(g_{t}^{-1} A g_{t}\right)(u)
\end{aligned}
$$

and hence

$$
\begin{aligned}
\dot{\nabla}(u) & =(\mathrm{D} \dot{g})(u)+\operatorname{ad}(A) \dot{g}(u) \\
& =((\mathrm{D}+\operatorname{ad}(A)) \dot{g})(u) \\
& =\left(\nabla^{\operatorname{End}(E)} \dot{g}\right)(u) .
\end{aligned}
$$


Q.E.D.

We can restate the proposition as follows: Define

$$
\mathrm{d}_{\nabla}^{0}: \Omega^{0}(M, \text { End } \mathcal{L}) \rightarrow \Omega^{1}(M, \text { End } \mathcal{L})
$$

by

$$
\left(\mathrm{d}_{\nabla}^{0} \alpha\right)(X):=\nabla_{X} \alpha,
$$

where $\alpha$ is a section of $\mathcal{L}, X$ a vector field on $M$ and we identify $\Omega^{0}(M, \operatorname{End}(\mathcal{L}))$ with $\Gamma(\operatorname{End}(\mathcal{L}))$. Then an infinitesimal variation comes from a gauge change if and only if

$$
\dot{\nabla} \in \operatorname{Im}\left(\mathrm{d}_{\nabla}^{0}\right) .
$$

This looks very much like a coboundary condition and thus our candidate for the tangent space of $\operatorname{Rep}\left(\pi_{1}(S), G\right)$ at $[\nabla]$, the quotient

$$
\frac{\operatorname{ker} \mathrm{d}_{\nabla}^{1}}{\operatorname{Im} \mathrm{d}_{\nabla}^{0}}
$$

looks very much like a cohomology space. Of course, for such an interpretation we need to know that $\mathrm{d}_{\nabla}^{1} \circ \mathrm{d}_{\nabla}^{0}=0$. This is indeed the case:

Proposition 4.1.3 Let $\nabla$ be any (not-necessarily flat) connection and define $\mathrm{d}_{\nabla}^{0}$ and $\mathrm{d}_{\nabla}^{1}$ as above. Then $\mathrm{d}_{\nabla}^{1} \circ \mathrm{d}_{\nabla}^{0}=R^{\nabla}$ is precisely the curvature of $\nabla$. Thus $\mathrm{d}_{\nabla}^{1} \circ \mathrm{d}_{\nabla}^{0}=0$ if and only if $\nabla$ is flat.

Now we may allow ourselves to write

$$
H_{\nabla}^{1}(M):=\frac{\operatorname{ker} \mathrm{d}_{\nabla}^{1}}{\operatorname{Im} \mathrm{d}_{\nabla}^{0}}
$$

and to call it the first $\nabla$-twisted cohomology of $M$.

\subsubsection{De Rham version of the twisted cohomology}

We have seen in subsection 4.1.1 that a certain twisted cohomology group $H_{\nabla}^{1}(M)$ can be interpreted heuristically as some sort of tangent space to the representation variety at the point $[\nabla]$. This group is in fact only the first in a family of twisted cohomology groups, which we will now define. The study of these more general cohomology groups is not just for completeness: recall that in order to prove non-degeneracy of the intersection form in the untwisted cohomology group $H^{1}(S)$ of a surface we also needed the groups $H^{0}(S)$ and $H^{2}(S)$. Similarly, we will need also their twisted analogs in the sequel. In order to define these one needs to prove the following proposition: 
Proposition 4.1.4 Let $\nabla$ be a connection on a vector bundle $\pi: \mathcal{L} \rightarrow M$. Then there exists a unique family of linear differential operators

$$
\mathrm{d}_{\nabla}^{k}: \Omega^{k}(M, \mathcal{L}) \rightarrow \Omega^{k+1}(M, \mathcal{L})
$$

such that the following two properties are satisfied:

(i) If $\omega \in \Omega^{p}(M)$ and $\alpha \in \Omega^{q}(M, \mathcal{L})$, then

$$
\mathrm{d}_{\nabla}^{p+q}(\omega \wedge \alpha)=\mathrm{d}^{p} \omega \wedge \alpha+(-1)^{p} \omega \wedge \mathrm{d}_{\nabla}^{q} \alpha,
$$

where $\mathrm{d}^{p}$ denote the untwisted de Rham differential.

(ii) If $\alpha \in \Omega^{0}(M, \mathcal{L}) \cong \Gamma(\mathcal{L})$ then

$$
\mathrm{d}_{\nabla}^{0} \alpha=\nabla \alpha
$$

The proof of this Proposition is the same as the proof of Proposition 2.5.4: it follows from a simple recursion. The operators $\mathrm{d}_{\nabla}^{0}$ and $\mathrm{d}_{\nabla}^{1}$ coincide with the operators defined above. Using Proposition 4.1.3 and property (i) it is easy to see that $\mathrm{d}_{\nabla}^{k} \circ \mathrm{d}_{\nabla}^{k-1} \equiv 0$ for all $k$ if and only if $\nabla$ is flat. Thus we may define:

Definition 4.1.5 Let $\pi: \mathcal{L} \rightarrow M$ be a vector bundle over $M$ and let $\nabla$ be a flat connection on $\mathcal{L}$.

- An $\mathcal{L}$-valued $k$-form $\omega \in \Omega^{k}(M, \mathcal{L})$ is closed if $\mathrm{d}_{\nabla}^{k} \omega=0$.

- An $\mathcal{L}$-valued $k$-form $\omega \in \Omega^{k}(M, \mathcal{L})$ exact if there exists $\alpha \in \Omega^{k-1}(M, \mathcal{L})$ with $\omega=\mathrm{d}_{\nabla}^{k-1} \alpha$.

- The $\nabla$-twisted $k$-th de Rham cohomology group of $M$ is

$$
H_{\nabla}^{k}(M):=Z_{\nabla}^{k}(M) / B_{\nabla}^{k}(M)
$$

where $Z_{\nabla}^{k}(M)$ the space of closed $k$-forms on $M$ and by $B_{\nabla}^{k}(M)$ the space of exact ones.

- The $k$-th $\nabla$-twisted Betti number is the dimension

$$
\beta_{k}^{\nabla}:=\operatorname{dim} H_{\nabla}^{k}(M)
$$

- The $\nabla$-twisted Euler characteristic is the alternating sum.

$$
\chi_{\nabla}(M):=\sum_{i=0}^{\operatorname{dim} M}(-1)^{i} \beta_{i}^{\nabla}
$$


The notation is in the line of our convention that we drop the dependence on $\mathcal{L}$ from our notation and see $\nabla$ as a short hand for the pair $(\mathcal{L}, \nabla)$. If we want to be very precise or want to stress the dependence on $\mathcal{L}$ we shall occasionally write $H_{\nabla}^{k}(M, \mathcal{L}):=H_{\nabla}^{k}(M)$.

Example 4.1.6 1. If $\mathcal{L}=M \times R$ is the trivial line bundle over $M$ and $\nabla=\mathrm{D}$ is the trivial connection then $H_{\mathrm{D}}^{k}(M)=H_{d R}^{k}(M)$ is just the ordinary de Rham cohomology. Thus twisted cohomology generalises de Rham cohomology.

2. Let $\nabla$ be flat connection on a vector bundle $\mathcal{L}$ and $\rho$ the holonomy representation from $\pi_{1}\left(S, x_{0}\right)$ to $\operatorname{End}\left(\mathcal{L}_{x_{0}}\right)$. The space $H_{\nabla}^{0}(M)$ is the space of $\nabla$-parallel section of $\mathcal{L}$. Moreover, the map $\sigma \rightarrow \sigma\left(x_{0}\right)$ is an isomorphism from $H_{\nabla}^{0}(M)$ to

$$
\mathcal{L}_{x_{0}}^{\rho\left(\pi_{1}\left(S, x_{0}\right)\right)}:=\left\{u \in \mathcal{L}_{x_{0}} \mid \forall \gamma \in \pi_{1}\left(S, x_{0}\right), \rho(\gamma)(u)=u\right\} .
$$

In particular, $\beta_{0}^{\nabla}(M)=\operatorname{rank}(\mathcal{L})$ if and only if the representation is trivial.

So far our considerations work for arbitrary manifolds. Now let us return to the special case of compact oriented surfaces: Most of the results stated above for ordinary de Rham cohomology can be generalised to the twisted case. The following theorem summarises the main results. Again the proofs will use a combinatorial model of twisted cohomology (via ribbon graphs) and are deferred to Paragraph 4.2.4.

Theorem 4.1.7 Let $S \cong S_{g}$ be a compact oriented surface of genus $g$, let $\pi: \mathcal{L} \rightarrow S$ be a vector bundle and let $\nabla$ be a flat connection on $\mathcal{L}$.

(i) The spaces $H_{\nabla}^{k}(S)$ are finite-dimensional.

(ii) The $\nabla$-twisted Euler characteristic of $S$ is given by

$$
\chi_{\nabla}(S)=\operatorname{rk}(\mathcal{L})(2-2 g) .
$$

In particular it depends only on the genus of $S$ and the rank of $\mathcal{L}$, but not on the choice of connection.

(iii) If $\mathcal{L}^{*}$ is the dual bundle of $\mathcal{L}$ and $\nabla^{*}$ is the dual connection of $\nabla$, then the pairing

$$
i_{\nabla}: H_{\nabla}^{1}(S) \times H_{\nabla^{*}}^{1}(S) \rightarrow \mathbb{R}, \quad(\alpha, \beta) \mapsto \int_{S} \omega_{\alpha, \beta},
$$


where

$$
\omega_{\alpha, \beta}(X, Y):=\langle\alpha \wedge \beta\rangle(X, Y):=\langle\alpha(X) \mid \beta(Y)\rangle-\langle\alpha(Y) \mid \beta(X)\rangle,
$$

and $\langle\cdot \mid \cdot\rangle$ denote dual pairing, is non-degenerate.

Before we turn to the proof in the next sections, let us consider some applications of Theorem 4.1.7.

Example 4.1.8 Suppose $(\mathcal{L}, \nabla)$ is a vector bundle with flat connection over $S$ which is equipped with a parallel non degenerate inner product $\langle\cdot, \cdot\rangle$. Here, parallel means that

$$
X .\langle v, w\rangle=\left\langle\nabla_{X} v, w\right\rangle+\left\langle v, \nabla_{X} w\right\rangle .
$$

Then we can use $\langle\cdot, \cdot\rangle$ in order to identify $(\mathcal{L}, \nabla)$ and $\left(\mathcal{L}^{*}, \nabla^{*}\right)$. Thus we obtain a symplectic form

$$
i_{\nabla}: H_{\nabla}^{1}(\mathcal{L}) \times H_{\nabla}^{1}(\mathcal{L}) \rightarrow \mathbb{R}, \quad(\alpha, \beta) \mapsto \int_{S}\langle\alpha \wedge \beta\rangle,
$$

where

$$
\langle\alpha \wedge \beta\rangle(X, Y):=\langle\alpha(X), \beta(Y)\rangle-\langle\alpha(Y), \beta(X)\rangle .
$$

We will call $i_{\nabla}$ is called the $\nabla$-twisted intersection form on $H_{\nabla}^{1}(\mathcal{L})$. In the case of the trivial line bundle with the trivial connection we recover the usual intersection form and duality.

The following example will be central for our treatment of the representation variety:

Example 4.1.9 Let $S$ be a surface and $G$ be a Lie group and let $\mathfrak{g}$ be its Lie algebra. Let $\rho: \pi_{1}(S) \rightarrow G$ be a homomorphism which corresponds to a $G$ local system $\left(\left\{U_{i}\right\}_{i \in I},\left\{g_{i j}\right\}_{i, j \in I}\right)$ via the non-abelian Hurewicz theorem. Then $\left(\left\{U_{i}\right\}_{i \in I},\left\{\operatorname{ad}\left(g_{i j}\right)\right\}_{i, j \in I}\right)$ is a local system which defines a flat $G$-connection $\nabla_{\rho}$ on a vector bundle $\mathcal{L}_{\mathfrak{g}}$ over $S$ with fibre $\mathfrak{g}$.

This example is so important for us, that we introduce a special notation for it:

Definition 4.1.10 Let $\rho: \pi_{1}(S) \rightarrow G$ be a representation and define $\mathcal{L}_{\mathfrak{g}}$ and $\nabla_{\rho}$ as in Example 4.1.9. Then the twisted cohomology groups associated to the bundle with connection $\left(\mathcal{L}_{\mathfrak{g}}, \nabla_{\rho}\right)$ are denoted by

$$
H_{\rho}^{i}(\mathfrak{g}):=H_{\nabla_{\rho}}^{i}(S) .
$$


Given a group $G$ we write $Z(G)$ for its centre, and given a subgroup $H$ of $G$ we write

$$
Z_{G}(H):=\{g \in G \mid g h=h g \text { for all } h \in H\}
$$

for its centraliser in $G$.

Proposition 4.1.11 Suppose that in the situation of Example 4.1.9, the group $G$ has a discrete centre and that we have $Z_{G}\left(\rho\left(\pi_{1}(S)\right)\right)=Z(G)$. Then

$$
\begin{aligned}
H_{\rho}^{0}(\mathfrak{g}) & =H_{\rho}^{2}(\mathfrak{g})=\{0\}, \\
\operatorname{dim} H_{\rho}^{1}(\mathfrak{g}) & =\chi(S) \operatorname{dim} \mathfrak{g} .
\end{aligned}
$$

Proof: Suppose $H_{\rho}^{0}(\mathfrak{g}) \neq\{0\}$. Then there exists a nonzero map $\alpha: V \rightarrow \mathfrak{g}$ satisfying $\alpha\left(e^{+}\right)=g_{e} \alpha\left(e^{-}\right)$, where $e$ is any edge and $\left\{g_{e}\right\}_{e \in \mathcal{L}}$ is the discrete connection associated with $\rho$. This means that for any vertex $v$ the element $\alpha(v) \in \mathfrak{g}$ is invariant under the holonomy map

$$
\pi_{1}(S, v) \rightarrow \operatorname{Aut}(\mathfrak{g}) .
$$

As the holonomy map factors as

$$
\pi_{1}(S, v) \stackrel{\rho}{\rightarrow} G \stackrel{\operatorname{Ad}}{\longrightarrow} \operatorname{Aut}(\mathfrak{g}),
$$

this implies that the one-parameter subgroup $\exp (t \alpha(v))$ commutes with the image $\rho\left(\pi_{1}(S)\right)$ of $\pi_{1}(S)$. Thus $Z(G)=Z_{G}\left(\rho\left(\pi_{1}(S)\right)\right)$ is non-discrete, which is a contradiction. Therefore $H_{\rho}^{0}(\mathfrak{g})=\{0\}$ and also $H_{\rho}^{2}(\mathfrak{g})=\{0\}$ by a duality similar to (iii) of Theorem 4.1.7. Finally, the equation

$$
\operatorname{dim} H_{\rho}^{1}(\mathfrak{g})=\chi(S) \operatorname{dim} \mathfrak{g},
$$

follows from part (ii) of Theorem 4.1.7. Q.E.D.

\subsection{A combinatorial version}

In order to prove Theorem 4.1.7 in Paragraph 4.2.4, we need to produce a combinatorial version of the twisted cohomology. We choose a ribbon graph $\Gamma$ filling $\Sigma$. We associate to each edge $e$ the oriented one dimensional manifold which is the interior of the geometric realisation and which we shall denote $\hat{e}$. In the same way we associate to every face $f$, the interior of each face which we denote by $\hat{f}$. By convention $v=\hat{v}$ 


\subsubsection{The combinatorial complex}

In order to smooth up our exposition, we have to consider the set of $\Gamma_{0}$ of oriented vertices, as well as the set $\Gamma_{2}$ of oriented faces, even though in our context vertices and faces have a canonical orientation. We denote as usual $\bar{\alpha}$ the element $\alpha$ of $\Gamma_{i}$ with the opposite orientation. The boundary $\partial \alpha$ of an oriented element $\alpha$ of $\Gamma_{i}$ is a tuple of elements of $\Gamma_{i-1}$, possibly with repetition. For instance, if $e$ is an edge then

$$
\partial e=\left(e_{+}, \overline{e_{-}}\right) .
$$

\section{A covering}

We now consider for every $\alpha \in \Gamma_{i}$ a contractible open set $U_{\alpha}$ which is a neighbourhood of the interior $\hat{\alpha}$, that is a vertex, the interior of the edge and the face. We denote by $W_{i}$ the union of all the open sets $U_{\alpha}$ so that $\alpha$ is in $\Gamma_{i}$. Finally we choose for every pair $(\alpha, \beta)$ so that $\alpha \in \partial \beta$, open sets $U_{\alpha, \beta}$ homeomorphic to disks.

- $U_{\bar{\alpha}}=U_{\alpha}$ and $U_{\bar{e}, \bar{f}}=U_{e, f}$.

- $\forall i, \forall \alpha, \beta \in \Gamma_{i}, \quad U_{\alpha} \cap U_{\alpha}=\emptyset$.

- For every edge $e, U_{e} \cap W_{0}=U_{e^{+}, e} \sqcup U_{\bar{e}_{-}, e}$,

- For every edge $e, U_{e} \cap W_{1}=U_{e, f(e)} \sqcup U_{\bar{e}, f(\bar{e})}$, where $f(a)$ is the unique face containing the edge $a$ and $g$.

- For every face $f$, that $W_{1} \cap U_{f}$ is an annulus

\section{Vector spaces and homomorphisms}

We now are given a vector bundle $\mathcal{L}$ equipped with a flat connexion $\nabla$.

We consider the vector space $L_{\alpha}$, which consists of section parallel of $\left.\mathcal{L}\right|_{U_{\alpha}}$. Observe that we have a canonical trivialisation of $\left.\mathcal{L}\right|_{U_{\alpha}}$ by $L_{\alpha} \times U_{\alpha}$, and that $L_{\alpha}=L_{\bar{\alpha}}$.

Moreover, observe that for any pair $(\alpha, \beta)$ so that $\alpha \in \partial b e t a$, there is a natural isomorphism $i(\alpha, \beta)$ from $L_{\alpha}$ to $L_{\beta}$ : if $u$ is parallel section along $U_{\alpha}$, $i(\alpha, \beta) u$ is the unique parallel section along $U_{\beta}$ which coincides with $u$ on $U_{\alpha, \beta}$.

Exercise 4.2.1 Describe $i_{\alpha, \beta}$ using a trivialisation of the bundle at every vertex and the combinatorial connexion associated to $\nabla$. 


\section{A combinatorial complex}

We consider the complex defined by

$$
C_{\Gamma}^{i}=\left\{c^{i}: \Gamma_{i} \rightarrow \sqcup_{\alpha \in \Gamma_{i}} L_{\alpha} \mid c^{i}(\alpha) \in L_{\alpha} \text { and } c^{i}(\bar{\alpha})=-c^{i}(\alpha)\right\},
$$

and the coboundary operators $d$ by

$$
\mathrm{d}_{i}: C_{\Gamma}^{i} \rightarrow C_{\Gamma}^{i+1}, \quad \mathrm{~d}_{i} c^{i}\left(\beta_{i+1}\right)=\sum_{\alpha_{i} \in \partial \beta_{i+1}} i\left(\alpha_{i}, \beta_{i+1}\right) c^{i}\left(\alpha_{i}\right) .
$$

One checks that $\mathrm{d} \circ \mathrm{d}=0$. We define

$$
H_{\Gamma}^{i}(L)=\operatorname{Ker}\left(\mathrm{d}_{i}\right) / \operatorname{Im}\left(\mathrm{d}_{i-1}\right) .
$$

\subsubsection{The isomorphism Theorem}

In this section, we prove that the two versions of the cohomology that we have built are the same.

First we need to build a map between complexes. We associate to an $\omega \in \Omega^{i}(S ; L)$ the element $\hat{\omega}$ in $C^{i}$ defined by

$$
\omega\left(\alpha^{i}\right)=\int_{\alpha^{i}} \omega .
$$

The integration is understood in the canonical trivialisation of $\left.\mathcal{L}\right|_{U_{\alpha_{i}}}$ by $L_{\alpha_{i}}$, since we have an identification $\Omega^{i}\left(U_{\alpha_{i}} ; \mathcal{L}\right)=\Omega^{i}\left(U_{\alpha_{i}}\right) \otimes L_{\alpha_{i}}$. We now claim

\section{Proposition 4.2.2}

$$
\widehat{\mathrm{d} \omega}=\mathrm{d} \hat{\omega}
$$

Proof: This is an easy consequence of Stokes formula and we shall only check it when $i=1$. We do it with technical details that we shall omit in the sequel. Let $f$ be an element of $\Gamma_{2}$. Let $\partial f=\left\{e_{1}, \ldots, e_{n}\right\}$. We consider $f$ as a map from the closed disk $\mathbb{D}$ to $S$. We observe that we can write $\partial \mathbb{D}$ as a reunion of closed intervals $I_{i}$ so that $\left.f\right|_{I_{i}}$ is a parametrisation of the edge $e_{i}$. By construction, the induced bundle $f^{*} \mathcal{L}$ is trivialised as $L_{f} \times \mathcal{D}$. As a consequence, if $\omega \in \Omega^{1}(S, L)$, then $f^{*} \omega \in \Omega(D) \otimes L_{f}$. Now

$$
\begin{aligned}
\widehat{\mathrm{d} \omega}(f) & =\int_{f} \omega=\int_{\mathbb{D}} \mathrm{d} f^{*} \omega \\
& =\int_{\partial \mathbb{D}} f^{*} \omega \\
& =\sum_{i=1}^{i=n} \int_{I_{i}} f^{*} \omega .
\end{aligned}
$$


Finally we have to remark that

$$
\int_{I_{i}} f^{*} \omega=i\left(e_{i}, f\right) \int_{e_{i}} \omega
$$

Hence

$$
\widehat{\mathrm{d} \omega}(f)=\mathrm{d} \hat{\omega}(f)
$$

Q.E.D.

It follows from this identification that we have a natural map $u \mapsto \hat{u}$ from $H_{\nabla}^{i}(S, \mathcal{L})$ to $H_{\Gamma}^{i}(S, \mathcal{L})$ so that

$$
[\hat{\omega}]=\widehat{[\omega]}
$$

We now prove

Theorem 4.2.3 [IsOMORPhism THEOREM] The map $u \mapsto \hat{u}$ from $H_{\nabla}^{i}(S, \mathcal{L})$ to $H_{\Gamma}^{i}(S, \mathcal{L})$ is an isomorphism

Again, to shorten our exposition we only prove this result for $i=1$. We prove this in two steps: injectivity and surjectivity of this map

Proposition 4.2.4 The map $u \mapsto \hat{u}$ from $H_{\nabla}^{i}(S, \mathcal{L})$ to $H_{\Gamma}^{i}(S, \mathcal{L})$ is surjective.

Proof: We first prove that given $c^{1} \in C^{1}$, there exists a neighbourhood $U_{1}$ of $\Gamma$, and a 1 -form $\omega \in \Omega^{1}(S, L)$ so that

$$
\begin{aligned}
\hat{\omega} & =c^{1} \\
\left.\mathrm{~d}^{\nabla} \omega\right|_{W_{2}} & =0 .
\end{aligned}
$$

It suffices to show this for $c^{1}$ such that there exists an edge $e$ so that $c^{1}(\alpha)=0$ if $\alpha \neq e$. Let indeed $\varphi$ be a real valued function defined on $U_{e}$ so that $\varphi=1$ on a neighbourhood of $U_{e_{+}}$and $\varphi=1$ on a neighbourhood of $U_{e_{-}}$. We now consider

$$
\sigma=\varphi \cdot c^{1}(e) \in \Omega^{0}\left(U_{e} ; \mathcal{L}\right) .
$$

Observe that $\mathrm{d}^{\nabla} \sigma=0$ on $U_{e_{ \pm}}$. It follows that $\beta=\mathrm{d} \sigma$ can be extended smoothly to $W_{1}$ by zero outside $U_{e}$. If $\psi$ is a function with support in $W_{1}$ which is equal to 1 on a neighbourhood of $\Gamma$, then

$$
\omega=\psi \beta,
$$

extended by 0 outside $W^{1}$, fulfils our conditions. 
Finally let's suppose that $\mathrm{d} c^{1}=0$. Let $\gamma_{f}$ be a circle in $W_{f}=f \cap W_{2}$ homotopic to the annulus $U_{f} \cap W_{1}$. We then have

$$
\int_{\gamma_{f}} \omega=\mathrm{d} c^{1}(f)=0 .
$$

It follows that $\left.\omega\right|_{W_{f}}=\mathrm{d} \beta_{f}$. We extend $\beta_{f}$ to $U_{f}$ and therefore we have extended $\omega$ to a closed form on $U_{f}$. Performing this operation for every face $f$, we end up with a closed form $\omega$ so that $\hat{\omega}=c^{1}$. Hence $u \mapsto \hat{u}$ is indeed surjective. Q.E.D.

Proposition 4.2.5 The map $u \mapsto \hat{u}$ from $H_{\nabla}^{i}(S, \mathcal{L})$ to $H_{\Gamma}^{i}(S, \mathcal{L})$ is injective.

Proof: Let's assume that $\omega$ is such that $\hat{\omega}=\mathrm{d} c^{0}$. We wish to prove that $\omega$ is exact.

We proceed by steps again.

We first notice that we can as well assume that $\omega=0$ on $W_{0}$. Indeed $\omega$ is exact on a neighbourhood $U_{0}$ of $W_{0}$ :

$$
\left.\omega\right|_{U_{0}}=\mathrm{d} \alpha .
$$

Hence using a function $\varphi$ with support in $U_{0}$ and equal to 1 on $W_{0}$ we reduce to this case by considering

$$
\omega-\mathrm{d}^{\nabla}(\varphi \alpha)
$$

Now we show that we can as well assume that $\hat{\omega}=0$. Indeed, we choose a parallel section $\sigma$ on $W_{0}$ so that for every vertex $v$, we have $\sigma(v)=c^{0}(v)$. It follows that $\omega-\mathrm{d}^{\nabla} \varphi v$ satisfies the required condition.

Next we show that we can reduce to the case that $\omega=0$ on a neighbourhood of $\Gamma$. Indeed, for every edge $e,\left.\omega\right|_{U_{e}}=\mathrm{d} \alpha_{e}$, where $\alpha$ is parallel on $W_{0} \cap U_{e}$. We can choose $\alpha_{e}$ so that $\alpha_{e}=0$ on one of the connected component, hence also on the other since $\int_{e} \omega=\hat{\omega}(e)=0$. Therefore, smoothing again by a function $\psi$ with support in $W_{1}$ and equal to 1 on a neighbourhood of $\Gamma$, we get that

$$
\omega-\mathrm{d}\left(\sum_{e \in E} \alpha_{e}\right),
$$

satisfies the condition.

Finally, we observe that for every face $f,\left.\omega\right|_{f}=\mathrm{d} \beta_{f}$. By our condition $\beta_{f}$ is zero on a neighbourhood of $\Gamma$. It follows that we can choose $\beta_{f}$ to be zero on a (connected) neighbourhood of $\Gamma$. Finally we get, that

$$
\omega=\sum_{f \in F} \mathrm{~d} \beta_{f} .
$$




\subsubsection{Duality}

In this section, we give the two versions of Poincaré duality: one for the de Rham cohomology, one for the combinatorial version.

\section{Symplectic complexes}

Definition 4.2.6 We say a complex $C^{\bullet}: 0 \rightarrow C^{0} \stackrel{\mathrm{d}}{\rightarrow} C^{1} \stackrel{\mathrm{d}}{\rightarrow} C^{2}$ of degree 2 is symplectic, if we have a

- a symplectic form $\omega$ on $C^{1}$,

- a non degenerate pairing $\omega$ on $C^{0} \times C^{2}$ such that

$$
\omega\left(\mathrm{d} \alpha_{0}, \alpha_{1}\right)=\omega\left(\alpha_{0}, \mathrm{~d} \alpha_{1}\right) .
$$

Proposition 4.2.7 The first cohomology is a symplectic vector space with symplectic form $[\omega]$ such that

$$
[\omega]([\alpha],[\beta])=\omega(\alpha, \beta) .
$$

Proof: this follows at once from the fact that

$$
\operatorname{ker}\left(\mathrm{d}_{1}\right)^{o}=\operatorname{im}\left(\mathrm{d}_{0}\right),
$$

where $V^{o}$ denote the orthogonal with respect to $\omega$ of $V \subset C^{1}$. Q.E.D.

\section{The dual graph}

We realise geometrically the dual graph $\Gamma^{*}$ in $S$. We denote by $\alpha \mapsto \alpha^{*}$ the map from $\Gamma_{i}$ to $\gamma_{2-i}$. As far as the boundary is concerned, we observe that

$$
\begin{aligned}
& v \in \partial e \quad \Longrightarrow \quad \overline{e^{*}} \in \partial v^{*}, \\
& e \in \partial f \quad \Longrightarrow \quad f^{*} \in \partial e^{*} .
\end{aligned}
$$

If $\alpha \in \Gamma_{0}^{*}$, we choose a neighbourhood $U_{\alpha}^{*}$ as above, requiring furthermore that for $v \in \Gamma^{0}, U_{v} \subset U_{v^{*}}^{*}$, for $e \in \Gamma^{1}, U_{e} \cap U_{e^{*}}^{*}$ is contractible, and for $f \in \Gamma^{2}, U_{f^{*}}^{*} \subset U_{f}$. We consider the dual bundle $\mathcal{L}^{*}$ to $\mathcal{L}$ together with its dual connection. We now observe that we have the canonical identification

$$
\left(L_{\alpha}\right)^{*}=L_{\alpha^{*}}^{*}
$$


Let $C_{\Gamma}^{\bullet}$ be the complex associated to $\Gamma^{*}$ and Let $C_{\Gamma^{*}}^{\bullet}$ be the complex associated to $\Gamma^{*}$. We consider that pairing between $C_{\Gamma}^{n-i}$ and $C_{\Gamma_{*}}^{i}$ by

$$
\langle\alpha, \beta\rangle=\frac{1}{2} \sum_{\gamma \in \Gamma^{n-i}=\Gamma_{*}^{i}}\left\langle\alpha(\gamma) \mid \beta\left(\gamma^{*}\right)\right\rangle .
$$

Let now $C^{\bullet}=C_{\Gamma}^{\bullet} \oplus C_{\Gamma^{*}}^{\bullet}$. We consider the bilinear form $\Omega$ defined

- on $\left(C_{\Gamma}^{0} \oplus C_{\Gamma^{*}}^{0}\right) \times\left(C_{\Gamma}^{2} \oplus C_{\Gamma^{*}}^{2}\right)$ by

$$
\Omega\left(\left(c^{0}, c_{*}^{0}\right),\left(c^{2}, c_{*}^{2}\right)\right)=\left\langle c^{0}, c_{*}^{2}\right\rangle+\left\langle c^{2}, c_{*}^{0}\right\rangle .
$$

- on $\left(C_{\Gamma}^{1} \oplus C_{\Gamma^{*}}^{1}\right) \times\left(C_{\Gamma}^{1} \oplus C_{\Gamma^{*}}^{1}\right)$ by

$$
\Omega\left(\left(c^{1}, c_{*}^{1}\right),\left(a^{1}, a_{*}^{1}\right)\right)=\left\langle c^{1}, a_{*}^{1}\right\rangle-\left\langle a^{1}, c_{*}^{1}\right\rangle .
$$

We then have

Theorem 4.2.8 [Dunlity Theorem] The complex $C^{\bullet}$ equipped with $\Omega$ is a symplectic complex. Moreover, the corresponding symplectic structure on

$$
H^{1}\left(C^{\bullet}\right)=H_{\Gamma}^{1}(\mathcal{L}) \oplus H_{\Gamma_{*}}^{1}\left(\mathcal{L}^{*}\right),
$$

is given in the de Rham interpretation by

$$
\left.\Omega\left([\alpha],\left[\alpha_{*}\right]\right),\left([\beta],\left[\beta_{*}\right]\right)\right)=\int_{S}\left\langle\alpha \wedge \beta_{*}\right\rangle-\int_{S}\left\langle\beta \wedge \alpha_{*}\right\rangle,
$$

where $\left\langle\alpha \wedge \beta_{*}\right\rangle \in \Omega^{2}(S)$ is given by

$$
\forall X, Y \in \mathrm{T}_{s} S, \quad\left\langle\alpha \wedge \beta_{*}\right\rangle(X, Y)=\left\langle\alpha(X) \mid \beta_{*}(Y)\right\rangle-\left\langle\alpha(Y) \mid \beta_{*}(X)\right\rangle .
$$

Finally the bilinear form $\Omega$ defines a duality between the complex $C_{\Gamma^{*}}^{\bullet}$ and the complex to $C_{\Gamma}^{\bullet}$.

We split the proof in two parts :

\section{The ribbon graph complex is a symplectic complex}

We want to prove the first part of the theorem which amounts to the following proposition

Proposition 4.2.9 We have

$$
\begin{aligned}
& \left\langle\mathrm{d} c^{0}, c_{*}^{1}\right\rangle=-\left\langle c^{0}, \mathrm{~d} c_{*}^{1}\right\rangle . \\
& \left\langle c^{1}, \mathrm{~d} c_{*}^{0}\right\rangle=\left\langle\mathrm{d} c^{1}, c_{*}^{0}\right\rangle .
\end{aligned}
$$


ProOF: by definition

$$
\begin{aligned}
\left\langle 2 \mathrm{~d} c^{0}, c_{*}^{1}\right\rangle & =\sum_{e \in \Gamma_{1}}\left\langle\mathrm{~d} c^{0}(e), c_{*}^{1}(e)\right\rangle \\
& =\sum_{e \in \Gamma_{1}} \sum_{v \in \partial e}\left\langle c^{0}(v), c_{*}^{1}(e)\right\rangle
\end{aligned}
$$

We can now rearrange the sum and get

$$
\begin{aligned}
\left\langle 2 \mathrm{~d} c^{0}, c_{*}^{1}\right\rangle & =\sum_{v \in \Gamma_{0}}\left\langle c^{0}(v), \sum_{e \mid v \in \partial e} c_{*}^{1}\left(e^{*}\right)\right\rangle \\
& =-\sum_{v \in \Gamma_{0}=\Gamma_{*}^{2}}\left\langle c^{0}(v), \sum_{e^{*} \in \partial v^{*}} c_{*}^{1}\left(e^{*}\right)\right\rangle \\
& =-2\left\langle c^{0}, \mathrm{~d} c_{*}^{1}\right\rangle .
\end{aligned}
$$

The second equality have a symmetric proof. Q.E.D.

\section{The de Rham interpretation}

Again this amounts to the following

Proposition 4.2.10 Using the mapping $\alpha \rightarrow \hat{\alpha}$ from $\Omega^{\bullet}(\Sigma, \mathcal{L})$ to $C_{\Gamma}^{\bullet}$. We have

$$
\int_{S}\left\langle\alpha \wedge \eta^{*}\right\rangle=\left\langle\hat{\alpha}, \hat{\eta}^{*}\right\rangle
$$

PRoOF: Indeed, we decompose the integral along the oriented faces:

$$
\int_{S}\left\langle\alpha \wedge \eta^{*}\right\rangle=\sum_{f} \int_{U_{f}}\left\langle\alpha \wedge \eta^{*}\right\rangle .
$$

We now use the fact that $U_{f}$ is contractible and write, on each $U_{f}, \eta^{*}=\mathrm{d} \beta_{f}^{*}$. Using stokes formula, we obtain

$$
\int_{S}\left\langle\alpha \wedge \eta^{*}\right\rangle=-\sum_{f} \sum_{e \in \partial f} \int_{e}\left\langle\alpha \mid \beta_{f}^{*}\right\rangle .
$$

We note that every oriented edge $e$ appears exactly once on the boundary of a face $f(e)=\left(\left(e^{*}\right)_{-}\right)^{*}$. Rearranging, we get

$$
\begin{aligned}
\int_{S}\left\langle\alpha \wedge \eta^{*}\right\rangle & =-\sum_{e \in \Gamma_{1}} \int_{e}\left\langle\alpha \mid \beta_{f(e)}^{*}\right\rangle \\
& =\frac{1}{2} \sum_{e \in \Gamma_{1}} \int_{e}\left\langle\alpha \mid\left(\beta_{f(\bar{e})}^{*}-\beta_{f(e)}^{*}\right)\right\rangle
\end{aligned}
$$


We now observe that, $\beta_{f(e)}^{*}-\beta_{f(\bar{e})}$ is a parallel section along the edge $e$ and that

$$
\beta_{f(\bar{e})}-\beta_{f(e)}^{*}=\int_{e^{*}} \eta_{*}
$$

Hence

$$
\begin{aligned}
\int_{S}\left\langle\alpha \wedge \eta^{*}\right\rangle & =\frac{1}{2} \sum_{e}\left\langle\int_{e} \alpha \mid \int_{e^{*}} \eta_{*}\right\rangle \\
& =\frac{1}{2} \sum_{e}\left\langle\alpha(e) \mid \eta_{*}\left(e_{*}\right)\right\rangle \\
& =\left\langle\alpha \mid \eta_{*}\right\rangle
\end{aligned}
$$

\subsubsection{Proof of Theorem 4.1.7}

Now we can proof the first two parts of Theorem 4.1.7:

Proof of (i) - (ii). As the spaces $C_{\Gamma}^{k}(S)$ are finite-dimensional, the associated cohomology spaces are also finite-dimensional and by Lemma 2.5.17 the alternating sum of their dimensions is given by

$$
\operatorname{dim} C_{\nabla, \Gamma}^{0}(S)-\operatorname{dim} C_{\nabla, \Gamma}^{1}(S)+\operatorname{dim} C_{\nabla, \Gamma}^{2}(S)=\operatorname{rk}(E)(2-2 g) .
$$

Moreover by a standard exercise in linear Algebra, we have

$$
\begin{aligned}
& \operatorname{dim} C_{\nabla, \Gamma}^{0}(S)-\operatorname{dim} C_{\nabla, \Gamma}^{1}(S)+\operatorname{dim} C_{\nabla, \Gamma}^{2}(S) \\
= & \operatorname{dim} H_{\Gamma}^{0}(S, \mathcal{L})-\operatorname{dim} H_{\Gamma}^{1}(S, \mathcal{L})+\operatorname{dim} H_{\Gamma}^{2}(S, \mathcal{L}) .
\end{aligned}
$$

We conclude the proof of the first two parts by using the Isomorphism theorem 4.2.3.

The last part of the Theorem is a consequence of the Duality Theorem 4.2.8.

\subsection{Torsion}

We introduce of a finer topological invariant, namely the Reidemeister torsion.

Torsion is primarily an invariant of an acyclic complex that is a complex with zero cohomology. Here is the main idea. Assume that we have a complex "defined over $\mathbb{Z}$ " by which we mean a complex of free $\mathbb{Z}$ modules, which gives rise to a complex of vector spaces after tensoring by $\mathbb{R}$. The complex may be acyclic as a complex of vector spaces, but not as a complex of $\mathbb{Z}$-modules. Here is the simplest example: we consider the differential $d$ from $\mathbb{Z}$ to $\mathbb{Z}$ given by $z \mapsto 2 . z$ and we complete by zero on both sides:

$$
C^{\bullet}: 0 \stackrel{0}{\longrightarrow} \mathbb{Z} \stackrel{z \mapsto 2 z}{\longrightarrow} \mathbb{Z} \stackrel{0}{\longrightarrow} 0 .
$$


The cohomology of this complex is non zero; indeed $H^{1}\left(C^{\bullet}\right)=\mathbb{Z} / 2 \mathbb{Z}$. However, when we make a tensor product by $\mathbb{R}$, we obtain an acyclic complex of vector spaces with zero cohomology:

$$
C^{\bullet}: 0 \stackrel{0}{\longrightarrow} \mathbb{R} \stackrel{z \mapsto 2 z}{\longrightarrow} \mathbb{R} \stackrel{0}{\longrightarrow} 0 .
$$

The remarkable point is that we can actually recover some of the lost information about the cohomology of the underlying complex over $\mathbb{Z}$ using very little structure on the vector spaces. Indeed, if we start with a free $\mathbb{Z}$-module and tensor it by $\mathbb{R}$, the resulting vector space has a natural volume form: the volume form whose least value is 1 on integer bases. When we go to complexes, the determinant of the differential is related to the cohomology of the $\mathbb{Z}$-complex. Let us see that in our example: the determinant of the differential of the complex (4.15) is 2 , and 2 is also the order of the cohomology of the complex (4.14).

In general, the Reidemeister torsion is an invariant of complexes with volume forms. For an acyclic complex that comes from $\mathbb{Z}$-modules, this invariant is a number which is related to the order of the cohomology of the $\mathbb{Z}$-modules which, in this case, are finite groups.

Our main objective here is not to make this relation precise, but first to define the torsion and to check in our topological case that it does not depend on the choices made. We will also relate in the our topological case of twisted cohomology the torsion - which is then a is a volume form in the cohomology - to the intersection form.

In the next chapters, this relation will be a useful tool.

\subsubsection{Determinants}

Definition 4.3.1 If $E$ is a finite dimensional vector space, we define $\operatorname{det}(E)=$ $\Lambda^{\operatorname{dim}(E)} E$ and $\operatorname{det}(E)^{-1}=\Lambda^{\operatorname{dim}(E)} E^{*}$. Let $C^{\bullet}: 0 \rightarrow C^{0} \rightarrow \ldots \rightarrow C^{n} \rightarrow 0$ be a complex. We define the determinant of the complex

$$
\operatorname{det}\left(C^{\bullet}\right)=\bigotimes \operatorname{det}\left(C^{i}\right)^{(-1)^{i}}
$$

Observe that

$$
\operatorname{det}\left(B^{\bullet} \oplus C^{\bullet}\right)=\operatorname{det}\left(B^{\bullet}\right) \otimes \operatorname{det}\left(C^{\bullet}\right)
$$

\subsubsection{An isomorphism between determinants}

Proposition 4.3.2 Let $C^{\bullet}$ a complex. Let $H^{\bullet}$ be its cohomology. There is a unique canonical isomorphism

$$
\tau_{C} \bullet: \operatorname{det}\left(C^{\bullet}\right) \rightarrow \operatorname{det}\left(H^{\bullet}\right)
$$


which satisfies

1. if $A^{\bullet}=B^{\bullet} \oplus C^{\bullet}$, then $\tau_{A} \bullet=\tau_{B} \bullet \otimes \tau_{C} \bullet$,

2. let $C^{\bullet}$ be the exact sequence $0 \rightarrow \ldots 0 \rightarrow C^{k} \stackrel{\mathrm{d}}{\rightarrow} C^{k+1} \rightarrow 0 \ldots \rightarrow 0$, whose cohomology is trivial, then

$$
\tau_{C} \bullet \operatorname{det}\left(C^{\bullet}\right)=\operatorname{det}\left(C^{k}\right)^{(-1)^{k}} \otimes \operatorname{det}\left(C^{1}\right)^{(-1)^{k+1}} \rightarrow \mathbb{R}
$$

is the canonical pairing $\tau_{C} \cdot(\alpha, \beta)=\left\langle\mathrm{d}_{*} \alpha \mid \beta\right\rangle$.

Proof: We indicate the sketch of the proof. Let us begin our construction. Any short exact sequence

$$
\nu: 0 \rightarrow A \stackrel{f}{\rightarrow} B \stackrel{g}{\rightarrow} C \rightarrow 0,
$$

Gives rise to an isomorphism $\tau_{\nu}$,

$$
\left\{\begin{aligned}
\operatorname{det}(A) \otimes \operatorname{det}(B)^{*} \otimes \operatorname{det}(C) & \rightarrow \mathbb{R} \\
a \otimes b \otimes c & \mapsto\langle b \mid f(a) \wedge \sigma(c)\rangle,
\end{aligned}\right.
$$

where $\sigma: C \rightarrow B$ is any map such that $g \circ \sigma=\mathrm{Id}$. It follows that $\tau_{\nu}$ - in the case $C=0$ satisfies the second property of the proposition. This construction extends easily in two ways:

1. first, to any acyclic complex

$$
C^{\bullet}: 0 \rightarrow C^{0} \stackrel{\mathrm{d}}{\rightarrow} C^{1} \stackrel{\mathrm{d}}{\rightarrow} \ldots \stackrel{\mathrm{d}}{\rightarrow} C^{n} \rightarrow 0
$$

where it gives rise to a an isomorphism $\tau_{C} \bullet: \operatorname{det}\left(C^{\bullet}\right) \rightarrow \mathbb{R}$.

2. to any short exact sequence of complexes $\xi: 0 \rightarrow A^{\bullet} \rightarrow B^{\bullet} \rightarrow C^{\bullet} \rightarrow 0$ where it gives rise to map $\tau_{\xi}: \operatorname{det}\left(A^{\bullet}\right) \otimes \operatorname{det}\left(B^{\bullet}\right)^{-1} \otimes \operatorname{det}\left(C^{\bullet}\right) \rightarrow \mathbb{R}$.

Finally, we can write any complex as $C^{\bullet}=H^{\bullet} \oplus A^{\bullet}$ where $A^{\bullet}$ is acyclic and $H^{\bullet}$ is the cohomology of $C^{\bullet}$. Then $\operatorname{det}\left(C^{\bullet}\right)=\operatorname{det}\left(H^{\bullet}\right) \otimes \operatorname{det}\left(A^{\bullet}\right)$ and, using the isomorphism $\tau_{\mathcal{A}} \bullet$, we indeed obtain an isomorphism of $\operatorname{det}\left(C^{\bullet}\right)$ with $\operatorname{det}\left(H^{\bullet}\right)$. This isomorphism is actually independent of the way to write the splitting of $C^{\bullet}$. From this construction, it also follows that $\tau_{C} \bullet B^{\bullet}=\tau_{B} \bullet \otimes \tau_{C} \bullet$.

The reader is invited to check the uniqueness. Q.E.D. 


\subsubsection{The torsion of a metric complex}

Definition 4.3.3 We say a complex $C^{\bullet}$ is metric if we have a non degenerate metric on each $C^{i}$. In this context we define the torsion $\operatorname{Tor}\left(C^{\bullet}\right) \in \operatorname{det}\left(H^{\bullet}\right)$ of the metric complex to be the image by $\tau$ of the volume forms on each of this $C^{i}$. Since we have not chosen any orientation, the torsion is defined only up to sign.

It follows from the construction of the torsion that if we have a short exact sequence of metric vector spaces,

$$
\xi: 0 \rightarrow D \stackrel{f}{\rightarrow} B \stackrel{g}{\rightarrow} C \rightarrow 0
$$

such that $f$ is an isometry then we have the following equality of real numbers,

$$
\operatorname{Tor}(\xi)=\operatorname{det}([g])^{-1},
$$

where $[g]$ is the quotient map from $B / f(D)$ - endowed with the quotient volume form - to $C$.

\subsubsection{The torsion of a flat metric connexion}

Let $\nabla$ be a flat connection preserving a non degenerate metric on a vector bundle $\mathcal{L}$ over $S$. Let $\Gamma$ be a ribbon graph. Observe first that the simplicial complex $C_{\Gamma}^{\bullet}$ is then a metric complex equipped with the metric

$$
g\left(a_{i}, b_{i}\right)=\frac{1}{2} \sum_{\alpha_{i} \in \Gamma_{i}} g\left(a_{i}\left(\alpha_{i}\right), b_{i}\left(\alpha_{i}\right)\right) .
$$

We prove

Theorem 4.3.4 [REIDEMEISTER TORSION] Let $\nabla$ be a flat connection preserving a non degenerate metric $g$ on a vector bundle $\mathcal{L}$ over $S$. Let $\Gamma$ be a ribbon graph and $C_{\Gamma}^{\bullet}$ the associated metric complex. Then the torsion $\operatorname{Tor}_{g}\left(C_{\Gamma}^{\bullet}\right)$ of this metric complex as an element of $\operatorname{det}\left(H_{\nabla}^{\bullet}(\mathcal{L})\right)$ does not depend of $\Gamma$. It is called the torsion of the connexion or the Reidemeister torsion and denoted $\operatorname{Tor}_{g}(\mathcal{L})$. Moreover, if $g$ and $h$ are parallel metrics with the same volume form then $\operatorname{Tor}_{g}(\mathcal{L})=\operatorname{Tor}_{h}(\mathcal{L})$

PROOF: Since two ribbon graphs can be considered as subgraphs of the same graph, it suffices to show the theorem whenever $\Gamma_{a}$ is a subgraph of $\Gamma_{b}$ then $\tau_{\Gamma_{0}}=\tau_{\Gamma_{b}}$. This means that every edge, vertex, face of $\Gamma_{a}$ can be written as 
union of edges, vertices, faces of $\Gamma_{b}$. This leads to a restriction map $R$ from $C_{\Gamma_{b}}^{\bullet}$ to $C_{\Gamma_{a}}^{\bullet}$. For instance if a face $f$ of $\Gamma_{a}$ splits as two faces $\left(f_{1}, f_{2}\right)$ of $\Gamma_{b}$ and if $c \in C_{\Gamma_{b}}^{2}$, we write

$$
R(c)(f)=c\left(f_{1}\right)+c\left(f_{2}\right) .
$$

By Theorem 4.2.3 - or a direct check that we shall actually perform soon this restriction map gives rise to an isomorphism at the cohomology level.

We want to understand the complex $D$ given by the kernel of $R$. It suffices to understand the two following elementary cases which correspond to elementary subdivision.

1. $\Gamma_{b}$ is obtained from $\Gamma_{a}$ by adding a vertex $v$ on an edge $e$, splitting it in two new edges $e_{1}$ and $e_{2}$. In this case,

- $D^{0}=\{c \mid \forall w \neq v, c(w)=0\}$

- $D^{1}=\left\{c \mid \forall e \notin\left\{e_{1}, e_{2}\right\}, c(e)=0\right.$ and $\left.c\left(e_{1}\right)+c\left(e_{2}\right)=0\right\}$

- $D^{2}=\{0\}$

In this case the metric restricted to $D^{\bullet}$ is non degenerate. Moreover, the determinant of $d$ from $D^{0}$ to $D^{1}$ is $\sqrt{2}^{\operatorname{rank}(\mathcal{L})}$. Hence $\operatorname{Tor}\left(D^{\bullet}\right)=\sqrt{2}^{\operatorname{rank}(\mathcal{L})}$.

2. $\Gamma_{b}$ is obtained from $\Gamma_{a}$ by adding an edge $e$ between two - not necessarily - distinct vertices $v_{1}$ and $v_{2}$, splitting a face $f$ in two faces $f_{1}$ and $f_{2}$.

- $D^{0}=\{0\}$

- $D^{1}=\{c \mid \forall \alpha \neq e, c(\alpha)=0\}$

- $D^{2}=\left\{c \mid \forall f \notin\left\{f_{1}, f_{2}\right\}, c(f)=0\right.$ and $\left.c\left(f_{1}\right)+c\left(f_{2}\right)=0\right\}$

In this case the metric restricted to $D^{\bullet}$ is also non degenerate. Moreover, the determinant of $d$ from $D^{1}$ to $D^{2}$ is $\sqrt{2}^{\operatorname{rank}(\mathcal{L})}$. Hence $\operatorname{Tor}\left(D^{\bullet}\right)=$ $\sqrt{2}^{-\operatorname{rank}(\mathcal{L})}$

We can also observe that $D$ is indeed acyclic and by the snake lemma[Hat02] $R$ is an isomorphism at the cohomology level. Now, let us compute the torsion of the short exact sequence of metric complexes

$$
\xi: 0 \rightarrow D \rightarrow C_{\Gamma_{b}}^{\bullet} \stackrel{R}{\rightarrow} C_{\Gamma_{a}}^{\bullet} \rightarrow 0 .
$$

Let $[R]^{\bullet}$ be the quotient map from $C_{\Gamma_{b}}^{\bullet} / D^{\bullet}$ to $C_{\Gamma_{b}}^{\bullet}$. Then

1. in the first case,

- $\operatorname{det}\left(\left[R^{0}\right]\right)=1$, 
- $\operatorname{det}\left(\left[R^{1}\right]\right)=\sqrt{2}^{\operatorname{rank}(\mathcal{L})}$,

- $\operatorname{det}\left(\left[R^{2}\right]\right)=1$.

It follows that $\operatorname{Tor}\left(\xi=\sqrt{2}^{\operatorname{rank}(\mathcal{L})}\right.$. On the other hand,

$$
\operatorname{Tor}\left(C_{\Gamma_{b}}^{\bullet}\right)=\frac{\operatorname{Tor}\left(D^{\bullet}\right)}{\operatorname{Tor}(\xi)} \operatorname{Tor}\left(C_{\Gamma_{a}}^{\bullet}\right) .
$$

Hence $\operatorname{Tor}\left(C_{\Gamma_{b}}^{\bullet}\right)=\operatorname{Tor}\left(C_{\Gamma_{a}}^{\bullet}\right)$.

2. Similarly, in the second case

- $\operatorname{det}\left(\left[R^{0}\right]\right)=1$,

- $\operatorname{det}\left(\left[R^{1}\right]\right)=1$,

- $\operatorname{det}\left(\left[R^{2}\right]\right)=\sqrt{2}^{\operatorname{rank}(\mathcal{L})}$.

It follows again that $\operatorname{Tor}\left(C_{\Gamma_{b}}^{\bullet}\right)=\operatorname{Tor}\left(C_{\Gamma_{a}}^{\bullet}\right)$.

We have shown that if $\Gamma_{a}$ is a subgraph of $\Gamma_{b}$ then

$$
\operatorname{Tor}\left(C_{\Gamma_{b}}^{\bullet}\right)=\operatorname{Tor}\left(C_{\Gamma_{a}}^{\bullet}\right)
$$

Q.E.D.

\subsubsection{Torsion and symplectic complexes}

Definition 4.3.5 We say a symplectic complex $C^{\bullet}$ is compatible with the metric, if the corresponding identifications of $C^{2-i}$ with $\left(C^{i}\right)^{*}$ given by the symplectic form is an isometry.

We then have

Proposition 4.3.6 Let $C^{\bullet}$ be a symplectic complex such that

$$
H^{0}\left(C^{\bullet}\right)=H^{2}\left(C^{\bullet}\right)=0,
$$

then the torsion of this complex equals the volume form associated to the symplectic form on $H^{1}\left(C^{\bullet}\right)$ :

$$
\operatorname{Tor}\left(C^{\bullet}\right)= \pm \frac{[\omega]^{d}}{d !}
$$

where $\operatorname{dim}\left(H^{1}\left(C^{\bullet}\right)\right)=2 d$. 
Proof: Let $g_{i}$ be the metric on $C^{i}$. Let $\omega$ be the symplectic form on $C^{1}$ and the pairing between $C^{0}$ and $C^{2}$ given in the definition of a symplectic complex.

Observe first that by definition of the compatibility of the metric and symplectic structure

$$
\frac{\omega^{c_{1}}}{c_{1} !}= \pm \operatorname{vol}\left(g_{1}\right)
$$

where $\operatorname{dim}\left(C^{1}\right)=2 c_{1}$. Let now $\left\{c_{a}^{i}\right\}_{i \in\left\{1, \ldots, \operatorname{dim}\left(C^{0}\right)\right\}}$ be an orthonormal basis of $C^{a}$, for $a \in\{0,2\}$.

Let $\sigma$ be a linear map from $C^{2}$ to $C^{1}$ so that $\sigma\left(C^{2}\right)$ is Lagrangian and $\mathrm{d} \circ \sigma=\mathrm{Id}$. Let $W$ be a symplectic vector subspace of $C^{1}$ so that

$$
\operatorname{ker}\left(\mathrm{d}_{1}\right)=\operatorname{im}\left(\mathrm{d}_{0}\right) \oplus W .
$$

Note that $W$ is isomorphic to $H^{1}\left(C^{\bullet}\right)$ as a symplectic vector space. The existence of $\sigma$ and $W$ follows from the definition of the symplectic complex. Let us now consider the acyclic complex

$$
D^{\bullet}: 0 \rightarrow C^{0} \rightarrow \operatorname{im}\left(\mathrm{d}_{0}\right) \oplus \sigma\left(C^{2}\right) \rightarrow C^{2} \rightarrow 0 .
$$

Observe that from the definition of a symplectic complex, the set

$$
\left\{\mathrm{d}_{0}\left(c_{0}^{i}\right), \sigma\left(c_{2}^{j}\right)\right\}_{i, j \in\left\{1, \ldots, \operatorname{dim}\left(C^{0}\right)\right.},
$$

forms a symplectic basis of $\operatorname{im}\left(\mathrm{d}_{0}\right) \oplus \sigma\left(C^{2}\right)$. Hence

$$
\tau_{D} \cdot\left(\bigwedge_{i} c_{0}^{i} \wedge \frac{\omega^{\operatorname{dim} C_{0}}}{\operatorname{dim}\left(C_{0}\right) !} \wedge \bigwedge c_{2}^{i}\right)=1 .
$$

We finally write

$$
C^{\bullet}=D^{\bullet} \oplus W^{\bullet},
$$

where

$$
W^{\bullet}: 0 \rightarrow 0 \rightarrow W \rightarrow 0 \rightarrow 0 .
$$

Using this splitting, it follows that

$$
\begin{aligned}
\operatorname{Tor}\left(C^{\bullet}\right) & =\tau_{C} \cdot\left(\bigwedge_{i} c_{0}^{i} \wedge \frac{\omega^{c_{1}}}{c_{1} !} \wedge \bigwedge c_{2}^{i}\right) \\
& =\tau_{D} \cdot\left(\bigwedge_{i} c_{0}^{i} \wedge \frac{\omega^{\operatorname{dim} C_{0}}}{\operatorname{dim} C_{0} !} \wedge \bigwedge c_{2}^{i}\right) \otimes \tau_{W} \cdot\left(\frac{\omega^{\frac{1}{2} \operatorname{dim}(W)}}{\left(\frac{\operatorname{dim}(W)}{2}\right) !}\right)
\end{aligned}
$$


To conclude the proof of the proposition, it suffices to remark that $W$ is isomorphic as a symplectic vector space to $H^{1}\left(C^{\bullet}\right)=H^{1}\left(W^{\bullet}\right)$. The result follows. Q.E.D.

We obtain as a corollary

Corollary 4.3.7 Let $\mathcal{L}$ equipped with a connexion $\nabla$ be a flat vector bundle. Assume that $H_{\nabla}^{0}(\mathcal{L})=H_{\nabla}^{2}(\mathcal{L})=\{0\}$. Note that $\mathcal{L} \oplus \mathcal{L}^{*}$ is equipped with a symmetric non degenerate metric $h$ given by

$$
h\left(\left(a, a^{*}\right),\left(b, b^{*}\right)\right)=\left\langle a \mid b^{*}\right\rangle+\left\langle b \mid a^{*}\right\rangle .
$$

Then, we have

$$
\operatorname{Tor}\left(\mathcal{L} \oplus \mathcal{L}^{*}\right)= \pm \frac{\Omega^{\operatorname{dim}\left(H_{\nabla}^{1}(\mathcal{L})\right)}}{\operatorname{dim}\left(H_{\nabla}^{1}(\mathcal{L})\right) !}
$$

where $\Omega$ is the symplectic form on $H_{\nabla}^{1}(\mathcal{L}) \oplus H_{\nabla}^{1}\left(\mathcal{L}^{*}\right)$ described in Theorem 4.2.8.

Proof: it follows at once from the definition of the symplectic structure on $C^{\bullet}=C_{\Gamma}^{\bullet} \oplus C_{\Gamma^{*}} \bullet$ by Formulae (4.7) and (4.8) that the metric is compatible with the symplectic structure.

\subsubsection{Parallel metric on bundles}

We now come to the main result of this section

Theorem 4.3.8 [TORSION AND SYMPLECTIC VOLUME] Let $\mathcal{L}$ be a vector bundle over $S$ equipped with a flat connexion and a parallel metric $g$. Assume that $H_{\nabla}^{0}(\mathcal{L})=H_{\nabla}^{2}(\mathcal{L})=0$. Let $\omega$ be the symplectic structure on $H_{\nabla}^{1}(\mathcal{L})$ given by

$$
\omega([\alpha],[\beta])=\int_{S} g(\alpha \wedge \beta)
$$

Then

$$
\operatorname{Tor}(\mathcal{L})=\frac{\omega^{\frac{1}{2} \operatorname{dim}\left(H_{\nabla}^{1}(\mathcal{L})\right)}}{\left(\frac{1}{2} \operatorname{dim}\left(H_{\nabla}^{1}(\mathcal{L})\right)\right) !} \in \Lambda^{\max }\left(H_{\nabla}^{1}(\mathcal{L})\right)
$$

REMARKS:

- This theorem was proved in [Wit91] for the case of positive metric. 
- Observe that Corollary 4.3.7 is a special case of this theorem. However, it will be the starting point of the proof.

- The proof of this theorem does not actually need the full strength of Theorem 4.3.4 but the immediate observation that the torsion of $C_{\Gamma}^{\bullet}$ is equal to the torsion of $C_{\Gamma^{*}}^{\bullet}$.

- This seemingly innocuous theorem will be used later in order to compute the volume of moduli spaces.

Proof: Let $p=\frac{1}{2} \operatorname{dim}\left(H_{\nabla}^{1}(\mathcal{L})\right)$. We use the corollary 4.3 .7 and its notations. In order to make our notations precise, we shall indicate by a subscript the metric with respect to which we compute the torsion. We first remark that the volume of $h$ and $g \oplus g^{*}$ coincide on $\mathcal{L} \oplus \mathcal{L}^{*}$. It follows that

$$
\operatorname{Tor}_{h}\left(\mathcal{L} \oplus \mathcal{L}^{*}\right)=\operatorname{Tor}_{g \oplus g^{*}}\left(\mathcal{L} \oplus \mathcal{L}^{*}\right)=\operatorname{Tor}_{g}(\mathcal{L}) \otimes \operatorname{Tor}_{g^{*}}\left(\mathcal{L}^{*}\right) .
$$

Let us use the metric to identify $\mathcal{L}$ and $\mathcal{L}^{*}$ and hence $H_{\nabla}^{1}(\mathcal{L})$ with $H_{\nabla}^{1}\left(\mathcal{L}^{*}\right)$. It follows from the duality Theorem 4.2.8 that

$$
\operatorname{Tor}_{g}(\mathcal{L})=\operatorname{Tor}_{g^{*}}\left(\mathcal{L}^{*}\right) .
$$

Let now $\lambda$ be the real number such that

$$
\operatorname{Tor}_{g}(\mathcal{L})=\operatorname{Tor}_{g^{*}}\left(\mathcal{L}^{*}\right)=\lambda \frac{\omega^{p}}{p ! 2^{p}} .
$$

By Assertions (4.16), (4.17) and the previous corollary, we obtain that

$$
\frac{1}{(2 p) !} \Omega^{2 p}=\lambda^{2}\left(\frac{1}{p !} i_{*}^{0} \omega^{p} \wedge \frac{1}{p !} i_{*}^{1} \omega^{p}\right)
$$

where $i_{0}: u \rightarrow(u, 0)$ and $i_{1}: u \rightarrow(0, u)$ are the two embeddings of $H^{1}(\mathcal{L})$ as factors in $H_{\nabla}^{1}(\mathcal{L}) \oplus H_{\nabla}^{1}\left(\mathcal{L}^{*}\right)$.

Finally, note that $j^{-}: u \rightarrow \frac{1}{\sqrt{2}}(u, u)$ and $j^{+}: u \rightarrow \frac{1}{\sqrt{2}}(u,-u)$ are symplectic embeddings of $H_{\nabla}^{1}(\mathcal{L})$ equipped with $\omega$ into $H_{\nabla}^{1}(\mathcal{L}) \oplus H_{\nabla^{*}}^{1}\left(\mathcal{L}^{*}\right)$ equipped with $\Omega$. Therefore

$$
\frac{1}{(2 p) !} \Omega^{2 p}=\lambda^{2}\left(\frac{1}{p !} i_{*}^{0} \omega^{p} \wedge \frac{1}{p !} i_{*}^{1} \omega^{p}\right)
$$

But det $\left(\left(j^{+}, j^{-}\right)^{-1} \circ\left(i_{0}, i_{1}\right)\right)=1$. This and Formulae (4.18) and (4.19) imply that $\lambda^{2}=1$. This completes the proof of the following formula

$$
\operatorname{Tor}_{g}(\mathcal{L})= \pm \frac{\omega^{p}}{p !}
$$

Q.E.D. 


\subsection{Comments, references and further read- ings}

Usually, the relation between combinatorial and de Rham twisted cohomology falls from general isomorphisms theorem. We chose here deliberately a down to earth approach in the context of surfaces.

Reidemeister torsion was introduced by Reidemeister in [Rei35]. In the context of de Rham cohomology, the torsion seems hard to define because the complex involved is infinite dimensional. Using regularised determinants and deeper analytical methods, Ray and Singer [RS71] defined an analytic torsion in a differentiable context which has been later on identified independently by Cheeger [Che77] and Müller [Mül78] to the Reidemeister torsion.

The notion of a symplectic complex and the subsequent identification of torsion with symplectic volumes is due to Witten in [Wit91]. 


\section{Chapter 5}

\section{Moduli spaces}

The purpose of this chapter is the study of the topology of the representation varieties $\operatorname{Rep}\left(\pi_{1}(S), G\right)$ for a compact oriented surface $S$ and a Lie group $G$. We will abbreviate

$$
\mathcal{M}(S, G):=\operatorname{Rep}\left(\pi_{1}(S), G\right) .
$$

Here the letter $\mathcal{M}$ stands for moduli space. We shall use this term as a synonym for representation variety. By the results of the previous chapters we may think of $\mathcal{M}(S, G)$ as a moduli space of flat connections or a moduli space of local systems or a moduli space of representations. As the term representation variety suggests that we are thinking of the latter model, we prefer to use the more general term moduli space unless we are really thinking of representations.

We first need to understand in which sense $\mathcal{M}(S, G)$ is a smooth manifold. We will introduce also the character variety although we shall not use the concept in the rest of the lectures. Then we will discuss the connected components of $\mathcal{M}(S, G)$ when $G$ is compact, then with $G=\operatorname{PSL}(2, \mathbb{R})$ proving Milnor-Wood Inequality and discussing further questions.

\subsection{Moduli space and character variety}

Recall that we are interested in the moduli space of representation

$$
\mathcal{M}(S, G)=\operatorname{Hom}\left(\pi_{1}(S), G\right) / G .
$$

In the sequel, we shall consider the case when $G$ is a group of matrices. This leads us to another definition. Let us introduce the following equivalence relation, let us say two homomorphisms $\rho_{1}$ and $\rho_{2}$ define the same character if for all elements $\gamma$ in $\pi_{1}(S)$,

$$
\operatorname{Tr}\left(\rho_{1}(\gamma)\right)=\operatorname{Tr}\left(\rho_{2}(\gamma)\right) .
$$


We then define a $G$-character to be a map

$$
\chi: \gamma \mapsto \operatorname{Tr}(\rho(\gamma))
$$

where $\rho$ is an element of $\operatorname{Hom}\left(\pi_{1}(S), G\right)$. This leads to the following definition Definition 5.1.1 The character variety $\chi(S, G)$ is the set of $G$-characters. The character variety is the quotient of the space $\operatorname{Hom}\left(\pi_{1}(S), G\right)$ by the equivalence relation "defining the same character".

Observe that we have a natural projection from $\mathcal{M}(S, G)$ to $\chi(S, G)$. We shall later see in Corollary 5.3.7 (and the subsequent comments) that the projection in injective on a large set. We shall also see that when $G$ is compact then this projection is a bijection.

Exercise 5.1.2 1. Prove that the set $\chi(S, G)$ is Hausdorff.

2. Prove that if $G=\mathrm{SL}(2, \mathbb{R})$ then $\mathcal{M}(S, G) \neq \chi(S, G)$.

\subsection{Topology and smooth structure}

In the study of the moduli space $\mathcal{M}(S, G)$ we will need some elementary notions on (real) algebraic varieties which we summarise in the next definitions :

Definition 5.2.1 [AfFine ALGEBRAIC VARIETIEs] $A n$ affine algebraic variety (over $\mathbb{R}$ ) is a subset $\Sigma$ of $\mathbb{R}^{n}$ for which there exists a family of real polynomials $\left\{P_{i}\right\}_{i \in I}$ in $n$ variables such that

$$
\Sigma=\bigcap_{i \in I} P_{i}^{-1}(\{0\})
$$

From the smooth point of view, a real algebraic manifold is a finite union of submanifolds $V_{i}$ each of them of dimension $k$ for $k \in\{0, \ldots, p\}$. The submanifold $V_{p}$ is the set of smooth points.

We now specialise these definitions to groups.

Definition 5.2.2 [Algebraic Groups AND Almost ZARISKI DENSE SubGROUPS] A subgroup $G$ of $\mathrm{GL}(n, \mathbb{R})$ is a (real) algebraic group if it is an affine algebraic variety as a subset of $M_{n}(\mathbb{R}) \cong \mathbb{R}^{n^{2}}$. The Zariski closure $\bar{A}^{Z}$ of a subset $A \subset \mathbb{R}^{n}$ is

$$
\bar{A}^{Z}=\bigcap \Sigma,
$$

where the intersection is taken over all affine algebraic varieties $\Sigma$ containing $A$. The set $A$ is Zariski closed if $A=\bar{A}^{Z}$. A subgroup $\Gamma$ of a real algebraic group $G$ is said to be almost Zariski-dense in $G$ if the Zariski closure $\bar{\Gamma}^{Z}$ contains the identity component of $G$. 
The definition is of course very much ad hoc, but it is good enough for our purpose.

Up to now we have treated the moduli spaces $\mathcal{M}(S, G)$ only as a set without topology. We are now going to turn them into topological spaces. We make the following assumption:

Convention 5.2.3 From now on, the letter $S$ will always denote a compact connected surface (without boundary) of genus $g \geq 2$. Thus $S$ is neither a sphere nor a torus. The letter $G$ will denote an algebraic group whose only normal subgroups are either finite or with finite index.

In order to define a topology on $\mathcal{M}(S, G)$, we think of it as a space of discrete flat connections. Thus we fix a filling ribbon graph $\Gamma$ of $S$ with vertices $V$, edges $E$ and faces $F$ and think of $\mathcal{M}(S, G)$ as

$$
\mathcal{M}(S, G)=\mathcal{D}_{\Gamma}(S, G) / G^{V}
$$

where

$$
\mathcal{D}_{\Gamma}(S, G)=\left\{\nabla \in G^{E} \mid \nabla \text { flat } G-\text { connection }\right\},
$$

denotes the set of discrete flat $G$-connections along $\Gamma$. If we equip $G^{E}=$ $\prod_{E} G$ with the product topology then the inclusion $\mathcal{D}_{\Gamma}(S, G) \subset G^{E}$ induces a topology $\tau$ on $\mathcal{D}_{\Gamma}(S, G)$. The set $\mathcal{D}_{\Gamma}(S, G)$ is defined inside $G^{E}$ by the vanishing of polynomials (namely $\nabla(e) \nabla(\bar{e})-1=0$ for each edge $e$ and $\mathrm{Hol}_{f}(\nabla)=0$ for each face $f$ ), and these polynomials and their derivatives have no common zeros. Moreover if $\bar{\Gamma}$ is a refinement of $\Gamma$, the restriction map sends $\mathcal{D}_{\Gamma}(S, G)$ to $\mathcal{D}_{\Gamma}(S, G)$ in an equivariant way. Summarising, we get

Proposition 5.2.4 The subspace $\mathcal{D}_{\Gamma}(S, G) \subset G^{E}$ is an affine subvariety. Moreover, the induced topology on $\mathcal{M}_{\Gamma}(S, G)$ is independent on the choice of $\Gamma$.

The topology on $\mathcal{D}_{\Gamma}(S, G)$ induces a topology on the quotient $\mathcal{M}(S, G)$. If the action of the gauge group $G^{V}$ on $\mathcal{D}_{\Gamma}(S, G)$ were proper and free then this quotient would be a nice manifold as well. Unfortunately, it is not so and the space $\mathcal{M}(S, G)$ with the quotient topology is not even Hausdorff in general. In order to complete our understanding of $\mathcal{M}(S, G)$ we need introduce the following subset of $\mathcal{D}_{\Gamma}(S, G)$.

Definition 5.2.5 [ZARISKI DENSE PART] The Zariski dense part of the space of discrete flat connections is the subset

$$
\mathcal{D}_{\Gamma}^{z d}(S, G)=\left\{\nabla \in \mathcal{D}_{\Gamma}(S, G) \mid \rho_{\nabla}\left(\pi_{1}(S)\right) \text { almost Zariski-dense in } G\right\},
$$


We can now state our main result.

Theorem 5.2.6 [SMOоTH STRUCTURE OF The MOduli SPACE] Let $\Gamma$ be a ribbon graph.

(i) The set $\mathcal{D}_{\Gamma}^{z d}(S, G)$ is a Zariski-open non-empty subset of $\mathcal{D}_{\Gamma}(S, G)$ which is a submanifold.

(ii) The gauge group $G^{V}$ acts freely and properly on $\mathcal{D}_{\Gamma}^{z d}(S, G)$.

(iii) The quotient

$$
\mathcal{M}^{z d}(S, G):=\mathcal{D}_{\Gamma}^{z d}(S, G) / G^{V}
$$

is a manifold of dimension $-\chi(S) \operatorname{dim} G$ whose tangent space at the class of a representation $[\rho]$ is canonically identified with $H_{\rho}^{1}(\mathfrak{g})$.

(iv) The complement of $\mathcal{D}_{\Gamma}^{z d}(S, G)$ in $\mathcal{D}_{\Gamma}(S, G)$ is a finite union of $G^{V}$-orbits union of algebraic manifolds of the form $\mathcal{D}_{\Gamma}(S, H)$, where $H$ varies over the algebraic subgroups of $G$. It is a finite union of submanifolds of dimension strictly less than the dimension of $\mathcal{D}_{\Gamma}^{z d}(S, G)$.

In the next chapter, we shall explain in Theorem 6.1.4 how the smooth part of the moduli space describes smooth family of flat connections.

A way to visualise this theorem is the following: although $\mathcal{M}(S, G)$ is not a manifold, it admits a stratification by manifolds and there is one big open stratum given by the representations with almost Zariski dense image.

We warn the reader that in general, the Zariski dense part is not dense as it follows from the discussion in Section 5.5.

\subsection{Proof of the Theorem 5.2.6}

In the next preliminary paragraph, we describe almost Zariski dense subgroups in an algebraic group.

Then we prove Theorem 5.2.6 in several steps. We spend some time now to explain the structure of this proof. First we use the following

Convention 5.3.1 In what follows, we denote by $L_{0}$ the connected component of the identity of a group $L$. If $H$ is a subgroup of $L$, we denote by $Z(H)$ the centraliser of $H$ in $L$, that is the group of elements commuting with $H$.

Let us now introduce the following subsets of $\mathcal{D}_{\Gamma}(S, G)$ : 
Definition 5.3.2 [REgUlaR, VERY REgular PARTS] The regular part of $\mathcal{D}_{\Gamma}(S, G)$ is

$$
\mathcal{D}_{\Gamma}^{r}(S, G)=\left\{\nabla \in \mathcal{D}_{\Gamma}(S, G) \mid Z\left(\rho_{\nabla}\left(\pi_{1}(S)\right)\right) \text { is finite }\right\}
$$

The very regular part of $\mathcal{D}_{\Gamma}(S, G)$ is

$$
\mathcal{D}_{\Gamma}^{v r}(S, G)=\left\{\nabla \in \mathcal{D}_{\Gamma}(S, G) \mid Z\left(\rho_{\nabla}\left(\pi_{1}(S)\right)\right)=Z(G)\right\} .
$$

We observe that we have the following inclusions when $Z(G)=Z\left(G_{0}\right)$.

$$
\mathcal{D}_{\Gamma}^{z d}(S, G) \subset \mathcal{D}_{\Gamma}^{v r}(S, G) \subset \mathcal{D}_{\Gamma}^{r}(S, G) .
$$

Indeed, if $H$ is a group then $Z(Z(H))$ is an algebraic group that contains $H$. Thus, if $H$ is almost Zariski dense, $G_{0} \subset Z(Z(H))$. It follows that $Z(H)$ is included in $Z\left(G_{0}\right)=Z(G)$, hence equal. This proves the first inclusion, the second is obvious.

We prove Theorem 5.2.6 using the following steps:

1. In Paragraph 5.3.2, we study the holonomy map and prove in Proposition 5.3.10 that the very regular part is a smooth submanifold.

2. In Paragraph 5.3.3, we prove in Proposition 5.3.14 that the almost zariski dense part is non empty. This completes the proof of the first part of Theorem .

3. In Paragraph 5.3.4, we prove that the action of the gauge group is proper and free on the regular part.

4. In Paragraph 5.3.5, we identify the tangent space to $\mathcal{M}^{z d}(S, G)$ with a cohomology group.

5. In Paragraph 5.3.6, we study the complementary set to $\mathcal{D}_{\Gamma}^{z d}(S, G)$.

We will not give complete proofs in full generality. This would require more knowledge about the structure of Lie groups than what we have assumed in this series of lectures. Nevertheless we will give the complete proof for $G=\mathrm{SL}(n, \mathbb{R})$ and give some hints about how to complete the proofs in the setting of a general semi-simple group. 


\subsubsection{The Zariski closure of a group}

Here are some properties that we are going to use freely.

Proposition 5.3.3 1. An affine algebraic variety $\Sigma \subset \mathbb{R}^{n}$ has finitely many connected components when equipped with the topology induced from the metric topology on $\mathbb{R}^{n}$.

2. The Zariski closure of a group is a group.

3. An algebraic group is a submanifold in $\mathrm{SL}(n, \mathbb{R})$ and hence a Lie group.

4. If $G$ is algebraic, if $H$ is a subgroup of $G$, then the normaliser of $H$ is an algebraic subgroup of $G$.

Almost Zariski-dense subgroups admit the following nice characterisation. In this proposition $\operatorname{Gr}_{k}(\mathfrak{g})$ denotes the Grassmannian of $k$-planes in $\mathfrak{g}$.

Proposition 5.3.4 Let $G$ be an algebraic group in $\mathrm{SL}(n, \mathbb{R})$. We assume that $\mathfrak{g}$ does not contain any non trivial ideal. Let $\Lambda$ be an infinite group in $G$ generated by $\left\{a_{1}, \ldots, a_{n}\right\}$. Then the following are equivalent:

(i) $\Lambda$ is almost Zariski-dense in $G$.

(ii) For any $0<k<\operatorname{dim} \mathfrak{g}$ we have

$$
\bigcap_{i=1}^{n} \operatorname{Fix}_{k}\left(a_{i}\right)=\emptyset,
$$

where for $g \in G$ we denote

$$
\operatorname{Fix}_{k}(g):=\left\{W \in \operatorname{Gr}_{k}(\mathfrak{g}) \mid g . W=W\right\}
$$

We obtain as an immediate corollary:

Corollary 5.3.5 Let $G$ be an algebraic group in $\mathrm{SL}(n, \mathbb{R})$. We assume that $\mathfrak{g}$ does not contain any non trivial ideal. Let $\Lambda$ be an infinite group in $G$ generated by a finite subset $S$. Then the set of homomorphisms of $\Lambda$ in $G_{0}$ which are almost Zariski dense, seen as a subset of $G^{S}$, is a Zariski open subset.

Proof: Let $G_{0}$ be the identity component of $G$.

Assume that $\Lambda$ is not Zariski-dense in $G$ and let $H=\bar{\Lambda}^{Z}$ and $\mathfrak{h}$ be its Lie algebra. 
We first claim that $0<\operatorname{dim} \mathfrak{h}<\operatorname{dim} \mathfrak{g}$. Indeed, if $\operatorname{dim} \mathfrak{h}=\operatorname{dim} \mathfrak{g}$, then $H$ contains $G_{0}$ and thus $\Lambda$ is almost Zariski-dense. On the other hand, if $\operatorname{dim} \mathfrak{h}=0$, then $H$ is discrete. By Proposition 5.3.3, $H$ is finite. Since $H$ contains $\Lambda$, we obtain again a contradiction.

Let $k:=\operatorname{dim} \mathfrak{h}$. As $H$ contains $\Lambda$, the adjoint action of $\Lambda$ fixes $\mathfrak{h}$. Thus

$$
\mathfrak{h} \in \bigcap_{i=1}^{n} \operatorname{Fix}_{k}\left(a_{i}\right)
$$

which violates (ii).

Conversely assume that $\Lambda$ is almost Zariski-dense and that (ii) is violated. Then there exist $k$, with $0<k<\operatorname{dim} \mathfrak{g}$, and

$$
W \in \bigcap_{i=1}^{n} \operatorname{Fix}_{k}\left(a_{i}\right)
$$

Let $\Sigma$ be the stabiliser of $W$ in $G$

$$
\Sigma:=\{g \in G \mid \operatorname{Ad}(\mathrm{g}) \mathrm{W}=\mathrm{W}\} .
$$

We observe that $\Sigma$ is an algebraic group: indeed, we consider $\mathfrak{g}$, hence $W$, as a subspace of $M(n, \mathbb{R})$ and note that $\Sigma$ is the intersection of $G$ and the stabiliser of $W$ in $\operatorname{SL}(n, \mathbb{R})$. Since $\Sigma$ contains $\Lambda, \Sigma$ also contains $G_{0}$. Thus, $G_{0}$ stabilises $W$ Hence, by taking derivatives, for all $X \in \mathfrak{g}$,

$$
\operatorname{ad}(X) W \subset W
$$

which means that $W$ is an ideal. As $0<k<\operatorname{dim} \mathfrak{g}$ this ideal is non-trivial which contradicts the simplicity of $\mathfrak{g}$. Q.E.D.

We have also the following criterion for products of almost Zariski-dense representations to be almost Zariski-dense:

Proposition 5.3.6 Let $G$ be an algebraic group with finite centre such that every normal subgroup is either finite or with finite index. Let $G_{0}$ be the connected component of the identity. Let $\Lambda$ be a group and let $\rho_{1}: \Lambda \rightarrow G_{0}$ and $\rho_{2}: \Lambda \rightarrow G_{0}$ be two representations with almost Zariski-dense image. Then

- either there exists an automorphism $h$ of $G_{0}$ such that $\rho_{1}$ and $h \circ \rho_{2}$ are equal,

- or $\rho_{1} \times \rho_{2}: \Lambda \rightarrow G \times G$ has also an almost Zariski-dense image. 
Proof: Let $H$ be the Zariski closure of $\left(\rho_{1} \times \rho_{2}\right)(\Lambda)$. Let $\pi_{1}$ and $\pi_{2}$ be the projections from $G \times G$ to the first factor and second factor.

We first prove that $\pi_{i}\left(H_{0}\right)=G_{0}$. First observe that the connected group $\pi_{i}\left(H_{0}\right)=G_{0}$ contains $\rho_{i}(\Lambda)$ and is thus non trivial. As a consequence, $\mathfrak{l}_{i}:=$ $T \pi_{i}\left(\mathfrak{h}_{0}\right)$, where $\mathfrak{h}_{0}$ is the Lie algebra of $H_{0}$, is non trivial. Since $\mathfrak{l}_{i}$ is normalised by the adjoint action of $\rho_{i}(\Lambda)$ and the latter is almost Zariski dense, it follows that $\mathfrak{l}_{i}$ is the full Lie algebra of $G_{0}$. Hence $\pi_{i}$ is open, thus surjective.

We now prove that $\pi_{i}$ is a covering. Let's consider $V=H_{0} \cap G_{0} \times\{e\}$.

Our first claim is that $V$ is either finite or $V=G_{0}$. Observe that $V$ is normalised by $H$, hence by $\left(\rho_{1} \times \rho_{2}\right)(\Lambda)$, hence by $\rho_{1}(\Lambda)$ since the second factor acts trivially on $V$. It follows by Zariski density of $\rho_{1}(\Lambda)$ that $V$ is a normal subgroup of $G$. Our claim follows by our hypothesis on $G$.

If $V=G_{0}$, since $\pi_{2}$ is surjective, it follows that $G_{0} \times G_{0} \subset H$ hence that $\rho_{1} \times \rho_{2}(\Lambda)$ is almost Zariski dense.

Assume now that $V$ is finite. This implies that $\pi_{1}$ and $\pi_{2}$ are finite coverings. They give rise to isomorphisms of $H_{0} / Z\left(H_{0}\right)$ with $G_{0} / Z\left(G_{0}\right)$. Therefore there exists an automorphism $g$ of $G / Z(G)$ such that

$$
H_{0} / Z\left(H_{0}\right)=\left\{(x, g(x)) \in G_{0} / Z\left(G_{0}\right) \times G_{0} / Z\left(G_{0}\right)\right\} .
$$

We can lift $g$ to an automorphism $h$ of $G_{0}$. It follows the graph of $h$ and $H_{0}$ are two connected subgroups of finite index of $\pi^{-1}\left(H_{0} / Z\left(H_{0}\right)\right)$ where $\pi$ is the projection from $G \times G$ to $G / Z(G) \times G / Z(G)$. Hence these two groups are equal. This finishes the proof. Q.E.D.

We will only use the following special case of this:

Corollary 5.3.7 Let $G$ be as in the previous proposition. Let $\rho_{1}: \Lambda \rightarrow G_{0}$ and $\rho_{2}: \Lambda \rightarrow G_{0}$ be two representations with almost Zariski-dense image. If $\operatorname{Tr} \rho_{1}(\gamma)=\operatorname{Tr} \rho_{2}(\gamma)$ for all $\gamma \in \Lambda$ then $\rho_{1}$ and $\rho_{2}$ are conjugate by an automorphism in $G_{0}$.

In particular, the map from $\mathcal{M}^{z d}(S, G)$ to $\chi(S, G)$ is injective.

Proof: If the representations have the same traces then they are contained in a proper subvariety of $G \times G$ and thus not Zariski dense. Q.E.D.

Exercise 5.3.8 [MORE ABOUT THE CHARACTER VARIETy] As an exercise we extend the identification above. We say a representation is reductive if the Lie algebra of its Zariski closure is a product of Lie algebras which have no non trivial ideals. Let $\mathcal{M}^{\text {red }}(S, G)$ be the set of reductive representations

1. Show that the projection from $\mathcal{M}^{\text {red }}(S, G)$ to $\chi(S, G)$ is injective. 
2. Show that $\mathcal{M}^{\text {red }}(S, G)=\mathcal{M}(S, G)$ when $G$ is compact.

3. With more knowledge on Lie groups, one can show that given any homomorphism $f$ from any group $\Gamma$ to $G$, there exist a sequence $\left\{g_{n}\right\}_{n \in \mathbb{N}}$ so that $\left\{g_{n} . f . g_{n}^{-1}\right\}_{n \in \mathbb{N}}$ converges to a reductive homomorphism. Using this fact show that we have a bijection of $\mathcal{M}^{\text {red }}(S, G)$ with $\chi(S, G)$.

\subsubsection{More on the holonomy map}

Let us denote by $G^{|E|}$ the set of discrete $G$-connections, which are maps $\alpha \in G^{E}$ with $\alpha(\bar{e})=\alpha(e)^{-1}$ and let $\mu: G^{|E|} \rightarrow G^{F}$ be the holonomy map. Note that a discrete $G$-connection $\alpha$ is flat if and only if $\mu(\alpha)=1$. We claim that for $\alpha \in \mathcal{D}_{\Gamma}^{v r}(S, G)$ the tangent map

$$
\mathrm{T}_{\alpha} \mu: \mathrm{T}_{\alpha} G^{|E|} \rightarrow \mathrm{T}_{1} G^{F}
$$

is onto. Then (ii) will follow from some version of the implicit function theorem. Note that for any $g \in G$ we can identify $\mathrm{T}_{g} G$ with $\mathfrak{g}$; if we view the elements of $G$ and $\mathfrak{g}$ as matrices then this identification is given simply by $\dot{g} \mapsto \dot{g} g^{-1}$. Under this identification of $\mathrm{T}_{g} G$ with $\mathfrak{g}$ the map $\mathrm{T}_{\alpha} \mu$ becomes identified with a map

$$
\dot{\mu}: \mathfrak{g}^{|E|} \rightarrow \mathfrak{g}^{F} .
$$

Now one can identify the map $\dot{\mu}$ by checking the following proposition:

Proposition 5.3.9 For $\alpha \in \mathcal{D}_{\Gamma}^{v r}(S, G)$ the diagram

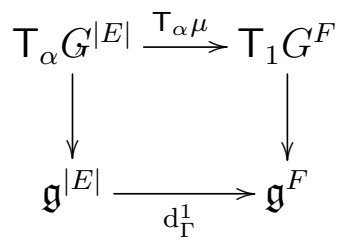

commutes. Thus $\dot{\mu}=\mathrm{d}_{\Gamma}^{1}$.

As a consequence, we have:

Proposition 5.3.10 The regular part $\mathcal{D}_{\Gamma}^{r}(S, G)$ is the set of regular points of $\mu$ in $\mathcal{D}_{\Gamma}(S, G)$. The regular part is an open Zariski set which is a submanifold.

PROOF: The regular part is easily shown to be a Zariski open set. Therefore the full proposition is a consequence of the first statement. By Proposition 5.3 .9 , we have to understand when $\mathrm{d}_{\Gamma}^{1}$ is surjective. By the definition of twisted 
cohomology $\mathrm{d}_{\Gamma}^{1}$ is surjective exactly when $H_{\Gamma}^{2}(\mathfrak{g})=\{0\}$. On the other hand by Poincaré duality $H_{\Gamma}^{2}(\mathfrak{g})=\{0\}$ is dual to $H_{\Gamma}^{0}(\mathfrak{g})=\{0\}$. Finally $H_{\Gamma}^{0}(\mathfrak{g})=$ $\{0\}$ is the Lie algebra of the centraliser of the holonomy. Hence the result follows.Q.E.D.

\section{Regular elements}

We need a refinement of the discussion in the last paragraph for $\Gamma=\Gamma_{g}$. We also need some properties of centralisers of elements. Let us begin with some definitions.

Definition 5.3.11 [Singular AND REgular Elements] Let $G$ be an algebraic group. We say an element $g$ of $G$ is singular if there exists a subgroup $K$ such that $\operatorname{dim} Z(K)>0$ and such that $g \in[K, K]$, and regular otherwise.

The reader is invited to check that for $\mathrm{SU}(2)$ the set of singular elements is the centre. A real split element in $\mathrm{SL}(n, \mathbb{R})$ is singular, if some product of eigenvalues is equal to 1 . We now prove

Proposition 5.3.12 Let $\mu$ be the map from $G^{2 g}$ to $G$ given by

$$
\left(a_{1}, b_{1}, \ldots, a_{g}, b_{g}\right) \mapsto \prod_{i=1}^{i=g}\left[a_{i}, b_{i}\right] .
$$

Then every regular element in $G$ is a regular value of $\mu$.

Proof: As a first computation, let $\nu$ be the map from $G^{k}$ to $G$,

$$
\left(c_{1}, \ldots, c_{k}\right) \rightarrow c_{1} \ldots c_{k} .
$$

Identifying $\mathrm{T}_{x} G$ with $\mathfrak{g}$ by the map $u \rightarrow g^{-1} . u$, we obtain that $\mathrm{T} \nu$ from $\mathfrak{g}^{k}$ to $\mathfrak{g}$ is given by

$$
\left(u_{1}, \ldots u_{k}\right) \mapsto \sum_{i=1}^{i=k}\left(\operatorname{ad}\left(c_{i+1} \ldots c_{k}\right)^{-1} u_{i}\right.
$$


A similar computation for $\mu$ yields that

$$
\begin{aligned}
& \mathrm{T} \mu\left(u_{1}, v_{1}, \ldots, u_{g}, v_{g}\right) \\
& =\sum_{i=1}^{i=g} \operatorname{ad}\left(\prod_{j>i}\left[a_{i}, b_{i}\right]\right)^{-1}\left(\operatorname{ad}\left(b_{i} a_{i} b_{i}^{-1}\right) \cdot u_{i}+\operatorname{ad}\left(b_{i} a_{i}\right) \cdot v_{i}-\operatorname{ad}\left(b_{i} a_{i}\right) \cdot u_{i}-\operatorname{ad}\left(b_{i}\right) \cdot v_{i}\right) \\
& =\sum_{i=1}^{i=g} \operatorname{ad}\left(\prod_{j>i}\left[a_{i}, b_{i}\right]\right)^{-1}\left(\operatorname{ad}\left(b_{i} a_{i} b_{i}^{-1}\right)\left(1-\operatorname{ad}\left(b_{i}\right)\right) u_{i}+\operatorname{ad}\left(b_{i}\right)\left(\operatorname{ad}\left(a_{i}\right)-1\right) \cdot v_{i}\right) \\
& =\sum_{i=1}^{i=g}\left(\operatorname{ad}\left(A_{i}\right)\left(1-\operatorname{ad}\left(b_{i}\right)\right) u_{i}+\operatorname{ad}\left(B_{i}\right)\left(\operatorname{ad}\left(a_{i}\right)-1\right) \cdot v_{i},\right.
\end{aligned}
$$

where

$$
A_{i}=\left(\prod_{j>i}\left[a_{i}, b_{i}\right]\right)^{-1} b_{i} \cdot a_{i} \cdot b_{i}^{-1}, \quad B_{i}=\left(\prod_{j>i}\left[a_{i}, b_{i}\right]\right)^{-1} b_{i} .
$$

Observe now that the image of the endomorphism of $\mathfrak{g}$ given by $u \rightarrow(1-$ $\operatorname{ad}(c)) u$ is the orthogonal - with respect to the ad invariant metric - of the lie algebra of $Z(c)$. It follows that the orthogonal of the image of $\mathrm{T} \mu$ is the Lie algebra of

$$
H=\bigcap_{i=1}^{i=g} Z\left(A_{i} a_{i} A_{i}^{-1}\right) \bigcap_{i=1}^{i=g} Z\left(B_{i} b_{i} B_{i}^{-1}\right)=Z\left\langle a_{1}, b_{1}, \ldots, a_{g}, b_{g}\right\rangle .
$$

It follows that $\left(a_{1}, b_{1}, \ldots, a_{g}, b_{g}\right)$ is a regular element for $\mu$ if and only if the centraliser of the group generated by these elements is finite.

Therefore, denoting $m=\left(a_{1}, \ldots, a_{g}\right)$ and $\Lambda(m)=\left\langle a_{1}, \ldots, a_{g}\right\rangle$, then $m$ is a singular point for $\mu$ if $\operatorname{dim}(Z(\Lambda(m)))>0$. Observe moreover that $\mu(m)$ belongs to $[\Lambda(m), \Lambda(m)]$. By definition, $\mu(m)$ is a a singular point in $G$. Q.E.D.

\subsubsection{The Zariski dense part is Zariski open and non- empty}

It follows from Corollary 5.3.5 that $\mathcal{D}_{\Gamma}^{z d}(S, G)$ is Zariski open. We will now prove that it is non empty. We will give a complete proof only for $G=\operatorname{SL}(n, \mathbb{R})$

Lemma 5.3.13 Let $G$ be a semi-simple algebraic group, then there exist two elements in $G$ that generates a Zariski dense group.

We will only prove this lemma for $G=\mathrm{SL}(n, \mathbb{R})$ and admits the result for general semi-simple groups. We say that a matrix $a \in \mathrm{SL}(n, \mathbb{R})$ is $\mathbb{R}$-split if it is diagonalisable with distinct real eigenvalues. 


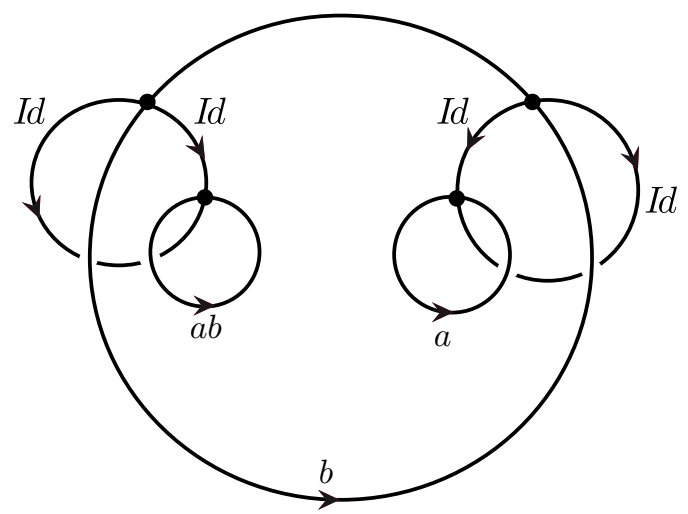

Figure 5.1: A flat discrete connection on a surface of genus 2

Proof: Let $a$ be any real split matrix. Suppose that, using the notation of Proposition 5.3.4, we have $W \in F i x_{k}(a)$. Then $W$ is generated by eigenvectors of $a$ (for the adjoint action): more precisely, if $\left\{e_{i}\right\}$ is the basis of eigenvectors of $a$ in $\mathbb{R}^{n}$, and $\left\{e^{i}\right\}$ the dual basis, then $W$ is generated by elements of the form $e^{j} \otimes e_{i}$. Thus by choosing the eigenvectors carefully it is possible to find another split matrix $b$ such that $\operatorname{Fix}_{k}(a) \cap F i x_{k}(b)=\emptyset$ for all $0<k<\operatorname{dim} \mathfrak{g}$. Then the lemma follows from Proposition 5.3.4. Q.E.D.

Now we can prove

Proposition 5.3.14 let $G$ be an algebraic semi-simple group. Let $S$ be a surface of genus greater than 1. Then $\mathcal{D}_{\Gamma}^{v r}(S, G)$ is non empty.

Proof: Choose $a$ and $b$ as in Lemma 5.3.13. For a pair of pants surface (in other words a sphere with three holes) one can explicitly write down a flat discrete connection such that the image of the holonomy representation contains $a$ and $b$ and is therefore Zariski-dense. For example one can take the flat discrete connection depicted in Figure 5.1.Gluing two pairs of pants with this discrete connection as in Figure 5.2 yields a discrete connection on a surface of genus 2, whose holonomy representation still contains $a$ and $b$ and thus is still very regular. If $S$ is a surface of genus $>2$ then we can decompose it along a disc into a surface of genus $g-1$ with one hole and a torus with one hole. Gluing connections as in Figure 5.2 we obtain a very regular connection on $S$ by induction on $g$. Q.E.D. 


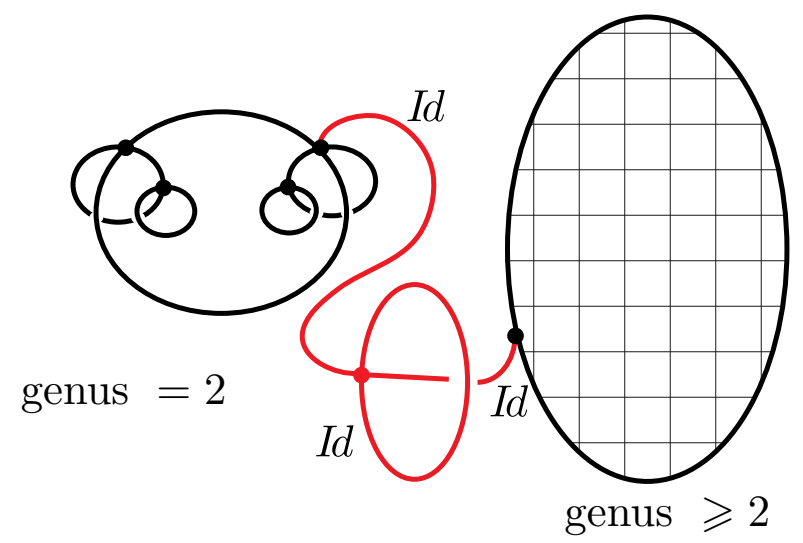

Figure 5.2: Very regular connections for higher genus

\subsubsection{The action is proper}

We prove the second part of Theorem 5.2.6

Proposition 5.3.15 The group $G^{V}$ acts freely and properly on $\mathcal{D}_{\Gamma}^{z d}(S, G)$.

The stabiliser of one element is identified with the centraliser of the holonomy. Hence the action is free on $\mathcal{D}_{\Gamma}^{v r}(S, G)$ which contains $\mathcal{D}_{\Gamma}^{z d}(S, G)$. The most difficult part is to verify that the $G$-action is proper. We cannot prove this in general here, so let us consider two special cases. The trivial case is the case where $G$ is compact. In this case, any action of $G$ is proper and there is nothing to show. In the non-compact case one needs again some more results on algebraic groups. An elementary proof with linear algebra methods is possible if we make two simplifications. Firstly, let us assume $\Gamma=\Gamma_{g}$. Then we have

$$
\mathcal{M}_{\Gamma_{g}}^{v r}(S, G)=\operatorname{Hom}^{v r}\left(\pi_{1}(S), G\right)
$$

and $G^{V}=G$. Thus the properness of the $G^{V}$-action is equivalent to the following statement:

(*) Let $\left\{\rho_{n}\right\}_{n \in \mathbb{N}} \subset \operatorname{Hom}^{v r}\left(A_{g}, G\right)$ be a convergent sequence. Then any sequence $\left\{g_{n}\right\}_{n \in \mathbb{N}} \in G$ for which $g_{n} \rho_{n} g_{n}^{-1}$ also converges, is bounded.

Now let us prove the statement $(*)$ and we shall only do it for $G=\operatorname{SL}(p, \mathbb{C})$. For this we can as well assume that $\rho_{n} \rightarrow \bar{\rho}_{0}$ and that $\left\{g_{n}\right\}_{n \in \mathbb{N}} \in G$ satisfies $g_{n} \rho_{n} g_{n}^{-1} \rightarrow \bar{\rho}_{1}$. We need two lemmas 
Lemma 5.3.16 There exist $a, b \in \pi_{1}(S)$ such that $\bar{\rho}_{0}(a), \bar{\rho}_{0}(b), \bar{\rho}_{1}(a), \bar{\rho}_{1}(b)$ are all $\mathbb{C}$-split and the axis' of $\bar{\rho}_{0}(a)$ (respectively $\bar{\rho}_{1}(a)$ ) are different from the axis' of $\bar{\rho}_{0}(b)$ (respectively $\bar{\rho}_{1}(b)$ ).

Proof: We distinguish two cases depending on whether $\bar{\rho}_{0}$ and $\bar{\rho}_{1}$ are conjugate or not. In the former case it suffices to choose $a$ and $b$ in such a way that $\bar{\rho}_{0}(a)$ and $\bar{\rho}_{0}(b)$ are $\mathbb{C}$-split with distinct axis. Now the set of $\mathbb{C}$-split elements of $G$ is Zariski-open and non-empty and thus contains an element $\bar{\rho}_{0}(a)$ form the Zariski-dense subset $\bar{\rho}_{0}\left(A_{g}\right)$. Moreover, the set of $\mathbb{C}$-split elements of $G$ with axis' different from those of $a$ is still Zariski open and this allows us to chose $b$. This settles the case where $\bar{\rho}_{0}$ and $\bar{\rho}_{1}$ are conjugate. In the other case it follows from Proposition 5.3.6 that $\left(\rho_{0} \times \rho_{1}\right)\left(\pi_{1}(S)\right)$ is Zariski dense in $G \times G$. Then the argument is reduced to the first case. Q.E.D.

Let us choose $a$ and $b$ as in the lemma. As $\rho_{n}(a) \rightarrow \bar{\rho}_{0}(a)$ and the set of $\mathbb{C}$-split elements in $G$ is open, we may assume that $\rho_{n}(a)$ is $\mathbb{C}$-split for all $n \in \mathbb{N}$. Let $\alpha_{n} \in\left(\mathbb{P}\left(\mathbb{C}^{p}\right)\right)^{p} / S^{p}$ denote the set of axis' of $\rho_{n}(a)$. Then $\alpha_{n} \rightarrow \bar{\alpha}_{0}$, where $\bar{\alpha}_{0}$ is the set of axis' of $\bar{\rho}_{0}(a)$. Similarly, $g_{n} \alpha_{n} \rightarrow \bar{\alpha}_{1}$, where $\bar{\alpha}_{1}$ is the set of axis' of $\bar{\rho}_{1}(a)$. The compact group $\mathrm{SU}(p) \subset \mathrm{SL}(p, \mathbb{C})$ acts transitively on the projective space and thus we can write

$$
g_{n}=t_{n} u_{n}
$$

where $u_{n} \in \mathrm{SU}(p)$ and $t_{n}$ is diagonal with respect to a basis of eigenvectors of $\bar{\alpha}_{1}$. As $\mathrm{SU}(p)$ is bounded we can reduce our discussion to the case where $g_{n}$ are diagonal matrices with respect to a basis of eigenvectors of $\bar{\alpha}_{1}$.

For that we need the following lemma:

Lemma 5.3.17 Let $\left\{g_{n}\right\}_{n \in \mathbb{N}}$ be an unbounded sequence of diagonal matrices in $\mathrm{SL}(p, \mathbb{C})$. Let $\left\{x_{n}\right\}_{n \in \mathbb{N}}$ be a sequence of elements of $\mathbb{P}\left(\mathbb{C}^{p}\right)$ which converges to an axis, which is not a coordinate axis. Then $\left\{g_{n} x_{n}\right\}_{n \in \mathbb{N}}$ converges to a coordinate axis.

Proof: We give the proof only for $p=2$. We then have $g_{n}=\operatorname{diag}\left(\lambda_{n}, \lambda_{n}^{-1}\right)$ and we may assume that $\lambda_{n} \rightarrow \infty$. If $x_{n}=\left[a_{n}: b_{n}\right]$ converges to $[a: b]$ with $a, b \neq 0$. Then

$$
g_{n} x_{n}=\left[\lambda_{n} a_{n}: \lambda_{n}^{-1} b_{n}\right] \rightarrow[1: 0] .
$$

Q.E.D.

Now we can finish the proof: Let $\beta_{n}, \bar{\beta}_{0}$ and $\bar{\beta}_{1}$ denote the set of axis' of $\rho_{n}(b), \bar{\rho}_{0}(b)$ and $\bar{\rho}_{1}(b)$ respectively. Then $g_{n} \beta_{n} \rightarrow \bar{\beta}_{1}$ and $\beta_{n} \rightarrow \bar{\beta}_{0}$. Write all 
elements of $G$ as matrices with respect to a basis of eigenvectors of $\bar{\alpha}_{1}$. Then the matrices $g_{n} \in \mathrm{SL}(p, \mathbb{C})$ are diagonal. Also as $\bar{\beta}_{0} \neq \bar{\alpha}_{1}, \beta_{n}$ converges to an axis', which is not a coordinate axis. Thus, by the lemma, if $g_{n}$ was unbounded then $g_{n} \beta_{n} \rightarrow \bar{\alpha}_{1}$, which would imply $\bar{\alpha}_{1}=\bar{\beta}_{1}$. But this contradicts the choice of $a$ and $b$. Therefore $\left\{g_{n}\right\}_{n \in \mathbb{N}}$ must be bounded. This finishes our indication of the proof of (iii).

Hint of the proof in the general case. We just sketch here how the general proof works. First we need the following fact about actions of algebraic groups: to show that an algebraic action of an algebraic group on an algebraic variety is proper, it suffices to show that the orbits are closed. Indeed, by Rosenlicht theorem [Ros63] the action is stratified in the following sense: there is a sequence of Zariski closed sets

$$
V_{n} \subset V_{n-1} \subset V_{n-2} \subset \ldots \subset V_{1} \subset V_{0}=V
$$

such that the action on $V_{i} \backslash V_{i+1}$ is proper.

In our case, the fact that the orbit are closed follows from Corollary 5.3.7: by this corollary, the $G$-orbit of a representation $\rho$, is

$$
V_{\rho}=\left\{\bar{\rho} \mid \forall \gamma \in \pi_{1}(S) \operatorname{Tr}(\rho(\gamma))=\operatorname{Tr}(\bar{\rho}(\gamma))\right\} .
$$

\subsubsection{The moduli space and its tangent space}

We prove the third part of Theorem 5.2.6.

Proposition 5.3.18 The quotient

$$
\mathcal{M}^{z d}(S, G):=\mathcal{D}_{\Gamma}^{v r}(S, G) / G^{V}
$$

is a manifold of dimension $-\chi(S) \operatorname{dim} G$ whose tangent space at the class of a representation $[\rho]$ is canonically identified with $H_{\rho}^{1}(\mathfrak{g})$.

Proof: We have already proved that $\mathcal{D}_{\Gamma}^{v r}(S, G)$ is a submanifold on which the group $G^{V}$ acts freely and properly. Hence the quotient is a submanifold and it remains to understand its tangent space.

Let us denote by $G^{|E|}$ the set of discrete $G$-connections, in other words maps $\alpha \in G^{E}$ with $\alpha(\bar{e})=\alpha(e)^{-1}$ and let $\mu: G^{|E|} \rightarrow G^{F}$ be the holonomy map.

Similarly, the action of $G$ gives rise to an orbit map $\mathfrak{g}^{V} \rightarrow \mathrm{T}_{\alpha} G^{|E|}$ and the complex

$$
\mathfrak{g}^{V} \rightarrow \mathrm{T}_{\alpha} G^{|E|} \rightarrow \mathrm{T}_{\mu(\alpha)} G^{F}
$$


can be identified using Proposition 5.3.9 with the complex

$$
\mathfrak{g}^{V} \stackrel{\mathrm{d}_{\Gamma}^{0}}{\longrightarrow} \mathfrak{g}^{E} \stackrel{\mathrm{d}_{\Gamma}^{1}}{\longrightarrow} \mathfrak{g}^{F} .
$$

Thus the tangent space at $[\alpha]$ becomes identified with the quotient of ker $\mathrm{d}_{\Gamma}^{1}$ by the image of $\mathrm{d}_{\Gamma}^{0}$, which is precisely $H_{\alpha}^{1}(\mathfrak{g})$.

It remains to compute the dimension of $\mathcal{M}^{z d}(S, G)$. We know that

$$
\operatorname{dim} \mathcal{M}^{z d}(S, G)=\operatorname{dim} H_{\rho}^{1}(\mathfrak{g}) .
$$

On the other hand, by using Proposition 4.1.11,

$$
\operatorname{dim}\left(H_{\Gamma}^{0}(\mathfrak{g})\right)-\operatorname{dim}\left(H_{\Gamma}^{2}(\mathfrak{g})\right)+\operatorname{dim}\left(H_{\Gamma}^{2}(\mathfrak{g})\right)=-\chi(S) \operatorname{dim}(\mathfrak{g}) .
$$

Since for elements in $\mathcal{D}^{v r}(S, G)$, the cohomology groups $H_{\Gamma}^{0}(\mathfrak{g}) H_{\Gamma}^{2}(\mathfrak{g})$ vanish, the formula for the dimension of $\mathcal{M}^{z d}(S, G)$ follows. Q.E.D.

\subsubsection{The complementary of the Zariski dense part}

We prove the final part of Theorem 5.2.6

Proposition 5.3.19 The complement of $\mathcal{D}_{\Gamma}^{z d}(S, G)$ in $\mathcal{D}_{\Gamma}^{z d}(S, G)$ is a finite union of $G^{V}$-orbits union of algebraic manifolds of the form $\mathcal{D}_{\Gamma}(S, H)$, where $H$ varies over the algebraic subgroups of $G$. It has Hausdorff dimension strictly less than the dimension of $\mathcal{D}_{\Gamma}^{z d}(S, G)$.

Proof: We only sketch the proof here. By construction, for every homomorphism $\rho$ not in $\mathcal{D}_{\Gamma}^{z d}$, there exists an algebraic group $H$ which contains $\rho(\Gamma)$. Hence, the first part of the statement follows from the following fact: there exists finitely many conjugacy classes of maximal algebraic subgroup of a semi-simple group. Q.E.D.

\subsection{Connected components}

\subsubsection{Connected components I: the compact case}

For general Lie groups $G$, the moduli space $\mathcal{M}(S, G)$ need not be connected. However, in some favourable cases this is indeed the case. For example one has:

Theorem 5.4.1 If $G$ is a compact, connected, simply-connected Lie group with finite centre, then $\mathcal{M}(S, G)$ is connected. 
We will only proof the theorem when $G=\mathrm{SU}(2)$. The general case requires a different proof from what we explain now:

To prove Theorem 5.4.1 we fix a genus $g$ and consider once again the map $\mu: G^{2 g} \rightarrow G$ given by $\left(a_{i}, b_{i}\right) \mapsto \prod\left[a_{i}, b_{i}\right]$. Then $\mathcal{M}(S, G)=\mu^{-1}(1) / G$ and thus $\mathcal{M}(S, G)$ will be connected if $\mu^{-1}(1)$ is connected.

Observe now that - in the case $G=\mathrm{SU}(2)$ - the set of singular elements is the centre of $G$.

It turns out that it is useful to study $\mu^{-1}(g)$ for $g \neq 1$ first. Let $T$ be the maximal abelian subgroup of $G$ - that is the group of diagonal matrices. Let $C$ be the set of elements $g$ such that $\operatorname{dim} Z(g)=\operatorname{dim} T$.

Lemma 5.4.2 The set $C$ is dense, connected and is simply connected.

PRoof: We can write

$$
C=\{g \in G \mid i \operatorname{Tr}(g) \in]-2,2[\} .
$$

Let $\varphi: g \rightarrow i \operatorname{Tr}(g)$. Note that $\varphi$ is a fibration from $C$ to its image and. Moreover the fibres of $\varphi$ restricted to $C$ are simply connected: they are homeomorphic to $\mathbb{C P}^{1}$. The lemma follows Q.E.D.

We also need the following lemma.

Lemma 5.4.3 The set $\mu^{-1}(C) \subset G^{2 g}$ is connected.

Proof: Let us first consider the set $R_{0}$ of elements which are critical points of $\mu$. An element $\rho$ is a critical point of $\mu$, if its centraliser is not finite. It follows that $R_{0}$ is included in the finite union of $G$-orbits of sets of the form $H^{2 g}$ where $H$ is a subgroup of $G$ of positive codimension. It follows that $R_{0}$ is included in a finite union of submanifolds of codimension greater than 2 . Hence $W=G^{2 g} \backslash R_{0}$ is open and connected. By definition $\mu$ restricted to $W$ is a submersion. Now $\mu^{-1}(C)=W \backslash \mu^{-1}\{\mathrm{Id},-\mathrm{Id}\}$. Let

$$
J=\mu^{-1}\{\operatorname{Id},-\operatorname{Id}\} \cap W
$$

has codimension equal to the dimension of $\mathrm{SU}(2)$ that is 3 . It follows that $\mu^{-1}(C)$ is connected. Q.E.D.

For a regular element the connectedness of the fibre is easy to prove:

Lemma 5.4.4 If $G=\mathrm{SU}(2)$ and $g \in C$, then $\mu^{-1}(g)$ is connected. 
Proof: Let $E:=\mu^{-1}(G \backslash C)$. Then the restriction $\mu: E \rightarrow G \backslash C$ is a fibration. By Lemma 5.4.2 the base $G \backslash C$ is simply connected and clearly $E$ is connected. Now the long exact homotopy sequence of $\mu$ ends with

$$
\ldots \rightarrow \underbrace{\pi_{1}(G \backslash C)}_{=\{0\}} \rightarrow \pi_{0}\left(\mu^{-1}(g)\right) \rightarrow \underbrace{\pi_{0}(E)}_{=\{0\}} \rightarrow \pi_{0}(G \backslash C) \rightarrow 0 .
$$

Thus $\pi_{0}\left(\mu^{-1}(g)\right)=\{0\}$ as claimed. Q.E.D.

Of course 1 is a very singular element. However, using the fact that the regular elements are dense, we can deduce Theorem 5.4.1 from the following approximation result:

Lemma 5.4.5 Let $K, P$ be topological spaces, $K$ compact and let $\mu: K \rightarrow P$ be a continuous map. Suppose that $\left\{x_{n}\right\}_{n \in \mathbb{N}}$ is a sequence in $K$ such that $\mu^{-1}\left(x_{n}\right)$ is connected for all $n \in \mathbb{N}$. If $x_{n} \rightarrow x_{0}$ then $\mu^{-1}\left(x_{0}\right)$ is also connected.

Exercise 5.4.6 Prove Lemma 5.4.5.

Now we may choose $K:=G^{2 g}, P=G, \mu$ as before and $x_{n}$ a sequence of regular elements converging to the identity in order to deduce Theorem 5.4.1.

\subsubsection{Connected Components II : an invariant}

Notice that the simple connectedness of $G$ entered crucially in the proof (via the long exact sequence of the fibration over the regular elements). Thus if we drop this assumption then it is not surprising that Theorem 5.4.1 fails. More precisely, let $G$ be a connected semisimple compact Lie group, not necessarily simply connected. Denote by $\pi: \widetilde{G} \rightarrow G$ its universal covering. We may identify $\pi_{1}(G)$ with the fibre over 1 . Then $\pi_{1}(G)$ becomes a normal discrete subgroup of $\widetilde{G}$ with $\widetilde{G} / \pi_{1}(G)=G$ and $\pi$ gets identified with the canonical quotient map.

Exercise 5.4.7 Show that a discrete normal subgroup $\Gamma$ of a connected topological group $H$ is central and deduce that $\pi_{1}(G) \subset Z(\widetilde{G})$. (Hint: For $\gamma \in \Gamma$ consider the conjugation map $c_{\gamma}: H \rightarrow \Gamma$ given by $h \mapsto h \gamma h^{-1}$.)

\section{Construction of the invariant}

We construct a map

$$
\chi: \mathcal{M}(S, G) \rightarrow \pi_{1}(G)
$$

constant on connected components. The construction depends on some choices and runs as follows. 
1. We fix a filling ribbon graph $\Gamma$ in $S$.

2. We choose a discrete flat connection on $\Gamma$, that is a collection $\nabla=$ $\left\{g_{e}\right\}_{e \in E}$ so that $g(\bar{e})=(g(e))^{-1}$, where $E$ denotes the edges of $\Gamma$, representing the element $[\nabla]$ of $\mathcal{M}(S, G)$.

3. For every $e \in E$ choose a lift $\widetilde{g_{e}} \in \widetilde{G}$ of $g_{e}$ in such a way that $\widetilde{g}_{\bar{e}}=\widetilde{g}_{e}^{-1}$. Then $\widetilde{\nabla}=\left\{\widetilde{g}_{e}\right\}_{e \in E}$ defines a non-flat discrete $\widetilde{G}$-connection on $\Gamma$.

4. For every face, we choose a vertex - called a base point - in the boundary of the face, , so that we can define the holonomy of a face.

If $F$ stands for the set of faces of $\Gamma$, we finally define

$$
\chi([\nabla]):=\prod_{f \in F} \operatorname{Hol}_{\widetilde{\nabla}}(f) .
$$

One has to check that this is well-defined and independent of the choices made. We first prove

Proposition 5.4.8 For every face $f$, the holonomy $\operatorname{Hol}_{\widetilde{\nabla}}(f)$ takes value in $\pi_{1}(G)$. Moreover

1. $\mathrm{Hol}_{\widetilde{\nabla}}(f)$ is independent of the choice of the base point of the face $f$,

2. $\prod_{f \in F} \operatorname{Hol}_{\widetilde{\nabla}}(f)$ is independent of the choice of the lift $\tilde{g}$,

3. $\prod_{f \in F} \operatorname{Hol}_{\widetilde{\nabla}}(f)$ is invariant under gauge transformations.

ProOF: First let us check that $\operatorname{Hol}_{\widetilde{\nabla}}(f)$ takes values in $\pi_{1}(G)$ : As $\nabla$ is flat, the holonomy $\operatorname{Hol}_{\nabla}(f)$ is trivial and thus $\pi\left(\operatorname{Hol}_{\tilde{\nabla}}(f)\right)=1$. Now let us check that $\mathrm{Hol}_{\widetilde{\nabla}}(f)$ does not depend on the choice of lift: indeed, if we take another lift $\widetilde{g}_{e}^{\prime}$ with $\widetilde{g}_{e}^{\prime}=\widetilde{g}_{e} \gamma$ for some $\gamma \in \pi_{1}(G)$, then we also have to take $\widetilde{g}_{\bar{e}}^{\prime}=\gamma^{-1} \widetilde{g}_{e}{ }^{-1}$. But by Exercise 5.4.7 the group $\pi_{1}(G)$ is abelian and thus $\gamma$ and $\gamma^{-1}$ cancel in the product over all faces. (Recall that every edge is contained in precisely one face.) Similarly, $\operatorname{Hol}_{\widetilde{\nabla}}(f)$ does not depend on the base point and is gaugeinvariant. We leave this to the reader.Q.E.D.

One finally checks that $\prod_{f \in F} \operatorname{Hol}_{\widetilde{\nabla}}(f)$ is invariant under subdivision and thus that it is independent of the choice of $\Gamma$. From this it follows that $\chi[\nabla]$ is well defined.

Now let us discuss the fibres of the map $\chi$. If we construct $\chi$ using the standard ribbon graph $\Gamma_{g}$, then it is easy to see that $\chi^{-1}(\alpha)$ is precisely the 
subset of the representation variety of the fundamental group of the punctured surface $\pi_{1}(S \backslash\{*\})$ with values in $\widetilde{G}$ given by the condition that the holonomy around the puncture is given by $\alpha$. Using the fact that $\widetilde{G}$ is simply-connected, a similar argument as in the proof of Theorem 5.4.1 will show that $\chi^{-1}(\alpha)$ is connected for all $\alpha$. This proves:

Theorem 5.4.9 If $G$ is a connected semisimple compact Lie group, then the connected components of $\mathcal{M}(S, G)$ are in one-to-one correspondence with the elements of $\pi_{1}(G)$.

Indeed, the correspondence is precisely given by the map $\chi$ above.

\subsubsection{Connected components III: Milnor-Wood inequal- ity}

For non-compact Lie groups the statement of Theorem 5.4.9 fails. This can already be seen in the simplest example: $G=\mathrm{SL}(2, \mathbb{R})$. Indeed, $\pi_{1}(\operatorname{PSL}(2, \mathbb{R})) \cong$ $\mathbb{Z}$ is infinite, and thus Theorem 5.4 .9 would predict an infinite number of connected components for $\mathcal{M}(S, G)$. However, this contradicts the fact that an algebraic variety - such as $\operatorname{Hom}^{z d}\left(\pi_{1}(S), G\right)$ - has only finitely many connected components. Therefore the problem to determine the number of connected components of $\mathcal{M}(S, G)$ cannot be solved by counting elements of the fundamental group unless $G$ is compact.

It also turns out that in some cases - and we shall only see the case $G=$ $\operatorname{PSL}(2, \mathbb{R})$ - the Euler class of the representation, which is a priori bounded by the argument above, can be explicitly bounded.

Theorem 5.4.10 [Milnor-Wood inequality] Let $G=\operatorname{PSL}(2, \mathbb{R})$. Then for any $[\nabla] \in \mathcal{M}(S, G)$,

$$
|\chi([\nabla])| \leq-\chi\left(S_{g}\right)=2 g-2 .
$$

\section{The universal cover of $\operatorname{PSL}(2, \mathbb{R})$}

We first observe that $\operatorname{PSL}(2, \mathbb{R})$ admits a retraction by deformation on the subgroup of rotation $\operatorname{SO}(2, \mathbb{R})$, since $\operatorname{PSL}(2, \mathbb{R}) / \operatorname{SO}(2, \mathbb{R})$ is the Poincaré disk. Thus

$$
\pi_{1}(\operatorname{PSL}(2, \mathbb{R}))=\pi_{1}(\mathrm{SO}(2, \mathbb{R}))=\mathbb{Z}
$$

Then, the universal cover of $\operatorname{PSL}(2, \mathbb{R})$ can be constructed as follows. Observe that $\operatorname{SL}(2, \mathbb{R})$ acts on the circle $\mathbb{T}^{1}$ through the identification with the projective line

$$
\mathbb{T}^{1} \sim \mathbb{R P}^{1}=\left(\mathbb{R}^{2} \backslash\{0\}\right) / \mathbb{R}^{*}
$$


Let now $\pi: \mathbb{R} \rightarrow \mathbb{T}^{1}$ be the universal covering of the circle given by $t \rightarrow$ $\exp (2 \pi i t)$. Let $\widetilde{G}$ be the subgroup of diffeomorphisms $\varphi$ of $\mathbb{R}$ lifting the action of $G=\operatorname{PSL}(2, \mathbb{R})$ :

$$
\widetilde{G}:=\{g \in \operatorname{Diff}(\mathbb{R}) \mid \exists h \in \operatorname{PSL}(2, \mathbb{R}), \pi \circ g=h \circ \pi\} .
$$

By construction, we have a group homomorphism $\pi^{*}$ from $\tilde{G}$ to $G=\operatorname{PSL}(2, \mathbb{R})$ and we have.

Proposition 5.4.11 The group $\tilde{G}$ is the universal cover of $G=\operatorname{PSL}(2, \mathbb{R})$ and $\pi^{*}$ is the covering map.

ProOf: We observe that the preimage of $\mathrm{SO}(2, \mathbb{R})$ is the group of translations. It follows from this observation that $\tilde{G}$ is connected and simply connected since it retracts on the group of translations. Q.E.D.

Proposition 5.4.12 Let $G=\operatorname{PSL}(2, \mathbb{R})$. There exists a - non continuous section $\sigma: G \rightarrow \widetilde{G}$ of the universal covering of $G$ such that for all $x \in \mathbb{R}$ and $g \in G$,

$$
|\sigma(g) \cdot x-x| \leq 1
$$

Proof: Assume first that $g$ has a a fixed point in $x_{0}$. Let $y_{0}$ a lift of $x_{0}$. We choose $\sigma(g)$ so that $\sigma(g)\left(y_{0}\right)=y_{0}$. Thus, since $\sigma(g)$ is increasing, we have $\sigma(g)\left[y_{0}, y_{0}+1\right]=\left[y_{0}, y_{0}+1\right]$. Thus, using the periodicity of $\sigma(g)$, Inequality (5.1) holds.

Assume now that $g$ has no fixed point. Let $\sigma(g)$ be a lift of $g$, so that $|\sigma(g)(0)| \leq \frac{1}{2}$. Since $x \rightarrow|\sigma(g)-x|$ is continuous and never takes an integer value - since $g$ has no foxed points, it follows that Inequality (5.1) holds. Q.E.D.

Proposition 5.4.13 For every $g$ and $h$ in $\operatorname{PSL}(2, \mathbb{R})$ and $x \in \mathbb{R}$,

$$
|[\sigma(g), \sigma(h)] x-x| \leq 2 .
$$

Proof: We first notice that since every element $\sigma(g)$ is a continuous lift of a diffeomorphism, we have

$$
|x-y| \leq 1 \Longrightarrow|\sigma(g)(x)-\sigma(g)(y)| \leq 1 .
$$

By Inequality (5.1)

$$
|\sigma(h)(z)-z| \leq 1
$$


Thus using Inequality (5.3), with $z=x$ and $y=\sigma(h)(x)$, we have

$$
\mid \sigma(g)(x)-\sigma(g)(\sigma(g)(x) \mid \leq 1 .
$$

Finally taking $y=\sigma(g)(x)$ in Inequality (5.4), we have

$$
\mid \sigma(g)(x)-\sigma(h)(\sigma(g)(x) \mid \leq 1 .
$$

Then,

$$
\begin{aligned}
\mid \sigma(g) \sigma(h)(x)-\sigma(h) \sigma(g)(x) \leq & |\sigma(h) \sigma(g)(x)-\sigma(g)(x)| \\
& +\mid \sigma(g)(x)-\sigma(h)(\sigma(g)(x) \mid \\
\leq & 2 .
\end{aligned}
$$

The Proposition follows. Q.E.D.

\section{A first inequality}

In order to bound the number of connected components of the moduli space we consider, as in the compact case, the map $\chi: \mathcal{M}(S, G) \rightarrow \pi_{1}(G) \cong \mathbb{Z}$. Then we have:

\section{Lemma 5.4.14 [FAKE MiLnor-WoOd INEQUALITY]}

Let $G=\mathrm{SL}(2, \mathbb{R})$. Then for any $[\nabla] \in \mathcal{M}(S, G)$,

$$
-2 g \leq \chi([\nabla]) \leq 2 g \text {. }
$$

ProOF: We compute $\chi$ via the standard ribbon graph $\Gamma_{g}$. Suppose $[\nabla]$ is represented by labellings $a_{1}, b_{1}, \ldots, a_{g}, b_{g} \in G$ at the $2 g$ edges. Let $\sigma$ be the section of the universal covering $\widetilde{G} \rightarrow G$ constructed in Proposition 5.4.12 and put $\widetilde{a_{i}}:=\sigma\left(a_{i}\right), \widetilde{b_{i}}:=\sigma\left(b_{i}\right)$. Then

$$
|\chi([\nabla])|=\prod_{i=1}^{g}\left|\left[\widetilde{a}_{i}, \widetilde{b_{i}}\right]\right| .
$$

We obtain inductively,

$$
\begin{aligned}
|\chi([\nabla]) x-x| \leq & \left.\mid\left[\widetilde{a_{1}}, b_{1}\right]\left[\widetilde{a_{2}}, b_{2}\right] \cdots\left[\widetilde{a_{g}}, b_{g}\right] x-\left[\widetilde{a_{2}}, b_{2}\right] \cdots\left[\widetilde{a_{g}}, b_{g}\right] x\right) \mid \\
& +\cdots+\left|\left[\widetilde{a_{g}}, b_{g}\right] \cdot x-x\right| .
\end{aligned}
$$

Now by Inequality (5.2), each of the $g$ summands is bounded by 2 and thus

$$
|\chi([\nabla]) x-x| \leq 2 g,
$$

from which the lemma follows. Q.E.D. 


\section{Proof of the Milnor-Wood inequality}

Actually, we can improve the bound on $|\chi([\nabla])|$ by "stabilising it" and get the Milnor-Wood inequality.

Proof: For any surface $S$ we have $|\chi(\nabla)| \leq \chi(S)+2$. Consider now an $n$ sheeted covering $S_{n} \rightarrow S$. View the elements of $\mathcal{M}(S, G)$ as equivalence classes of representations. Any representation $\rho: \pi_{1}(S) \rightarrow G$ lifts to a representation $\rho_{n}: \pi_{1}\left(S_{n}\right) \rightarrow G$. Now $\chi\left(\left[\rho_{n}\right]\right)=n \chi([\rho])$ since a preimage of a ribbon graph as $n$ times as many faces as the original ribbon graph and $\chi\left(S_{n}\right)=n \chi(S)$. Thus

$$
|\chi([\rho])|=\frac{1}{n} \chi\left(\left[\rho_{n}\right]\right) \leq \frac{1}{n}\left(-\chi\left(S_{n}\right)+2\right)=-\chi(S)+\frac{2}{n} .
$$

As $n$ was arbitrary, the theorem follows. Q.E.D.

\subsubsection{Connected components IV: Goldman's work for $\operatorname{PSL}(2, \mathbb{R})$}

For $\operatorname{PSL}(2, \mathbb{R})$ the situation of connected components is pretty well understood thanks to W. Goldman's work. More precisely, the fact that the Milnor-Wood inequality is sharp follows from the following theorem of Goldman [Gol88]:

Theorem 5.4.15 [Goldman] Let $G=\operatorname{PSL}(2, \mathbb{R})$. Then for all $p$ with $|p| \leq$ $-\chi(S)$ the fibre $\chi^{-1}(p)$ is connected and non-empty. Thus $\mathcal{M}(S, G)$ has precisely $4 g-3$ connected components.

The maximal component $\chi^{-1}(2 g-2)$ consists of discrete faithful representations. It can be identified with Teichmüller space, the space of oriented hyperbolisations of the surface $S$. (A discrete faithful representation yields a covering action of $\pi_{1}\left(S_{g}\right)$ on the upper half plane $\operatorname{PSL}(2, \mathbb{R}) / \operatorname{PSO}(2, \mathbb{R})$ and thus a hyperbolisation.) The minimal component $\chi^{-1}(-2 g+2)$ corresponds to the Teichmüller space of $S$ with opposite orientation. On these two components the action of the mapping class group, that is the group of outer automorphisms $\operatorname{Mod}_{g}:=\operatorname{Out}\left(\pi_{1}\left(S_{g}\right)\right)$, is proper. On the other hand one has the following conjecture of Goldman:

Conjecture 5.4.16 [Goldman] For every $p \notin\left\{ \pm \chi\left(S_{g}\right)\right\}$ the action of $\operatorname{Mod}_{g}$ on $\chi^{-1}(p)$ is ergodic.

Thus there is a stark contrast between the spaces of maximal/minimal representations and the other components of the moduli space. 


\subsection{Comments, references and further read- ings}

Since we did not assume the students have any knowledge of algebraic groups and algebraic actions, we only gave down to earth proof for $G=\operatorname{SL}(n, \mathbb{R})$. The description of the character variety and its smooth points can be found in full generality in Rapinschuk-Benyash-Krivets-Chernousov, [RBKC96], LubotzkyMagid [LM85] and incidentally in Goldman [Gol88, Gol84] and Bismut-Labourie [BL99].

The fact that the character variety is connected for simply connected compact or complex reductive groups is proved in Jun Li [Li93], it uses the identification of the character variety with stable holomorphic bundles due to Narasimhan-Seshadri (for the unitary case) [NS65] extended to the general case by Ramanathan [Ram75]. The proof given in these notes for $\mathrm{SU}(2)$ is new, but cannot be extended straightforwardly to all compact simply connected Lie groups. It would be nice nevertheless to have a proof in this spirit of the connectedness of $\mathcal{M}(S, G)$ in this spirit when $G$ is simply connected.

The remark than only finitely may values of characteristic classes can be obtained for flat $G$-bundles on a given manifold is attributed to Milnor. MilnorWood inequality is proved by Milnor for $\operatorname{PSL}(2, \mathbb{R})$ and Wood for circle bundles in [Woo71, Mil58]. Turaev [Tur84] extended this inequality for symplectic groups, and later Burger-Iozzi-Wienhard [BIW03] and Bradlow-GarcíaPrada-Gothen extended it to all isometry groups of hermitian symmetric spaces [BGPG06].-

The classification by Goldman of connected components for $G=\operatorname{PSL}(2, \mathbb{R})$ is in [Gol88]. Goldman's ergodicity conjecture about non maximal components for non $G=\operatorname{PSL}(2, \mathbb{R})$ is the pendant of his ergodicity theorem for $G=\mathrm{SU}(2)$ [Gol97].

The first examples of connected components with the same characteristic classes is due to Hitchin for $\operatorname{PSL}(n, \mathbb{R})$ [Hit92]. One of the most interesting case is the character variety for $G=\operatorname{Sp}(4, \mathbb{R})$ whose connected components have been counted by García-Prada-Mundet i Riera [GPiR04] and described topologically by Guichard-Wienhard [GW10]. Concerning connected components let us remark that it follows from Goldman-Millson Rigidity Theorem [GM87] that a connected component may contain no Zariski dense representation and have a smaller dimension than expected, thus Zariski dense representations are not always dense.

Atiyah-Bott [AB83] foundational paper - completed by Daskalopoulos in [Das92] - gives further topological information using a Morse theoretic approach, and this approach has lead to a lot of work. 


\section{Chapter 6}

\section{Symplectic structure of the moduli spaces}

In this chapter, we concentrate on the symplectic nature of the representation variety $\mathcal{M}(S, G)$. After constructing the symplectic structure, we describe several related Poisson algebras of observables: the Goldman algebra of Wilson loops and spin networks observables.

Finally, following Witten, we compute the volume of $\mathcal{M}(S, G)$ when $G$ is a compact Lie group.

\subsection{Universal connections and smooth struc- tures}

In this preliminary section, we relate the smooth part of the moduli space with smooth family of connections.

\subsubsection{Lifting Lemmas}

\section{Lifting small neighbourhoods}

For a bundle $E$ over $S$ denote by $\mathcal{A}_{E}$ the affine space of connections and by $A_{E}:=\Omega^{1}(S, \operatorname{End}(E))$ the underlying vector space.

Lemma 6.1.1 [Lifting Lemma] Let $\left[\rho_{0}\right]$ be an element of $\mathcal{M}^{z d}(S, G)$. Then there exist a neighbourhood $U$ of $\left[\rho_{0}\right]$ in $\mathcal{M}^{z d}(S, G)$, a bundle $E$ over $S$ and a smooth map $f: U \rightarrow \mathcal{A}_{E}$ whose image consists of flat connections so that the holonomy representation of $f([\rho])$ is given by $[\rho]$. 
ProOF: Fix a good covering of $S$. If $\mathcal{X}^{z d}$ denotes the space of $G$-local systems with respect to this covering, which represent Zariski dense representations, then the projection $\mathcal{X}^{z d} \rightarrow \mathcal{M}^{z d}(S, G)$ is a locally trivial principal bundle. In particular, it admits local sections. Thus we find a neighbourhood $U$ of $\left[\rho_{0}\right]$ and a section $\sigma: U \rightarrow \mathcal{X}^{z d}$.

We furthermore assume that $U$ is diffeomorphic to a ball.

For every point in $U$ we have a locally constant $G$-valued cocycle on $S$ with respect to our chosen covering. In other words, we have a $G$-valued cocycle on $S \times U$, which is locally constant along $S$. Observe that a cocycle give rises to a concrete bundle $E$ on $S \times U$ together with a flat $G$-connection along $S$. (Note that a local system really gives rise to a concrete bundle with connection, not just an equivalence class, and that it does so in a smooth way).

Now, it suffices to show that this bundle is "constant" along $U$, or more precisely that it is equivalent to the pullback of a bundle over $S$ via the projection $\pi: S \times U \rightarrow S$. This follows from the contractibility of $U$.

Let us explain this fact in some more details: Let $E_{0}:=\left.E\right|_{S \times\left\{\left[\rho_{0}\right]\right\}}$, which is a bundle over $S \times\left\{\left[\rho_{0}\right]\right\} \cong S$. Let $\widetilde{E}:=\pi_{0}^{*} E_{0}$, where $\pi_{0}: S \times U \rightarrow S \times\left\{\left[\rho_{0}\right]\right\}$ is the canonical projection. Our goal is to construct a isomorphism $F: E \rightarrow \widetilde{E}$ which induces the identity on the base space $S \times U$. For this, we take any connection on $E$ - without any relation whatsoever to any of the connections occurring before.

As $U$ is a ball with centre $\left[\rho_{0}\right]$, we can join every point $u \in U$ to $\left[\rho_{0}\right]$ by a path $c_{u}:[0,1] \rightarrow U$ so that the map $U \times[0,1] \rightarrow U$ given by $(u, t) \mapsto c_{u}(t)$ is smooth. The bundle isomorphism $F$ is defined by assigning to $v \in E_{(s, u)}$ its image by the holonomy along $c_{u}$ :

$$
F(v):=\operatorname{Hol}_{\{s\} \times c_{u}}(v) .
$$

Q.E.D.

As a byproduct we obtain the following result:

Corollary 6.1.2 If $\left[\nabla_{0}\right],\left[\nabla_{1}\right]$ are in the same connected component of the moduli space $\mathcal{M}^{z d}(S, G)$, then they can be represented by connections on the same bundle $E$.

\subsubsection{Universal connections}

We now elaborate on the Lifting Lemma and introduce the following device.

Definition 6.1.3 A universal connection is a connection $\nabla$ on a bundle $\mathcal{E}$ over $S \times \mathcal{M}^{z d}(S, G)$ such that for every representation $\rho$ belonging to $\mathcal{M}^{z d}(S, G)$, $\left.\nabla\right|_{\{[\rho]\} \times S}$ is a flat connection with holonomy conjugate to $\rho$. 
The following result is a generalisation of the Lifting Lemma.

Theorem 6.1.4 [Universal CONNECTION] For every surface $S$ and group $G$ there exists a universal connection $\nabla^{\sharp}$ over a bundle over $S \times \mathcal{M}^{z d}(S, G)$.

Proof: we use the notation and ideas of the proof of the Lifting Lemma.

The initial remark of this proof is that a smooth map from an open set $U$ to $\mathcal{A}_{E}$ is the same object as a smooth connection on $E \rightarrow S \times U$ which is trivial along $\{s\} \times U$.

Therefore, assume that we have two open sets $U$ and $V$ in $\mathcal{M}^{z d}(S, G)$. Let $f$ and $g$ be two lifts from respectively $U$ and $V$ to $\mathcal{A}_{E}$. Let $\nabla_{U}$ and $\nabla_{V}$ be the associated connections on $S \times U$ and $S \times V$ respectively. Then by hypothesis, for any $m$ in $U \cap V$, the connections $\left.\nabla_{U}\right|_{\{m\} \times S}$ and $\left.\nabla_{U}\right|_{\{m\} \times S}$ are gauge equivalent. Since the holonomies have Zariski dense image, the gauge equivalence is unique. In other words, there exists a unique $\sigma_{m}=\sigma_{m}^{U, V}$ in the gauge group of $E \rightarrow S$ so that

$$
\left.\nabla_{U}\right|_{\{m\} \times S}=\left.\sigma_{m}^{*} \nabla_{V}\right|_{\{m\} \times S} .
$$

From the uniqueness it follows that $m \rightarrow \sigma_{m}$ is continuous. Moreover, the construction given in the proof of the lifting Lemma yields that $m \rightarrow \sigma$ is smooth.

Let now $\left\{U_{i}\right\}_{i \in I}$ be a covering of $\mathcal{M}^{z d}(S, G)$ by open sets such as in the Lifting Lemma. From the uniqueness again, the collection of maps $\left\{\sigma_{m}^{U, V}\right.$ defines a $G$-valued cocycle on $E$ and a vector bundle $\mathcal{E}$ over $S \times \mathcal{M}^{z d}(S, G)$.

Moreover each connection $\nabla_{i}:=\nabla_{U_{i}}$ gives rise to a connection on $\left.\mathcal{E}\right|_{U_{i}}$ and by construction on $U_{i} \cap U_{j}$ we have by Equation 6.1

$$
\left.\nabla_{i}\right|_{\{m\} \times S}=\left.\nabla_{j}\right|_{\{m\} \times S} .
$$

We now produce a universal connection by setting

$$
\nabla=\sum_{i} \varphi_{i} \nabla_{i}
$$

where $\varphi_{i}$ is a partition of unity subordinated to the covering.

Q.E.D.

The final statement of this section is an elaboration on these ideas and follows from the proof. 
Proposition 6.1.5 Let $\nabla^{\sharp}$ be a universal connection. Let $M$ be a manifold, $E$ be a vector bundle over $M$ over $M \times S, \nabla$ be a connection on $E$ such that for any point $m$ in $M,\left.\nabla\right|_{\{m\} \times S}$ is a flat connection whose holonomy representation $[\rho]_{m}$ has almost Zariski dense image. Let $\Phi$ be the map from $M \times S$ to $\mathcal{M}^{z d}(S, G) \times S$ given by $(m, s) \rightarrow\left([\rho]_{m}, s\right)$. Then

1. The map $\Phi$ is smooth.

2. There exist an element $\sigma$ of the gauge group of $E$, so that for any $m$ in $M$,

$$
\left.\Phi^{*} \nabla^{\sharp}\right|_{\{m\} \times S}=\left.\sigma^{*} \nabla\right|_{\{m\} \times S} .
$$

\subsection{The symplectic form}

The aim of this subsection is to equip the Zariski dense part $\mathcal{M}^{z d}(S, G)$ of the moduli space with a symplectic structure in the sense of the following definition:

Definition 6.2.1 A symplectic structure on a manifold $M$ of dimension $2 n$ is a 2 -form $\omega \in \Omega^{2}(M)$ which satisfies

(i) $\mathrm{d} \omega=0$, in other words $\omega$ is closed;

(ii) $\omega$ is fibrewise non-degenerate, in other words $\omega^{\wedge n}$ is nowhere vanishing.

The pair $(M, \omega)$ is then called a symplectic manifold.

Recall from Theorem 5.2.6 that the tangent space $\mathrm{T}_{[\rho]} \mathcal{M}^{z d}(S, G)$ can be identified with the twisted cohomology group $H_{\rho}^{1}(\mathfrak{g})$. On $H_{\rho}^{1}(\mathfrak{g})$ the intersection form $i_{\rho}$ defines a non-degenerate alternating bilinear form. These forms fit together nicely:

Theorem 6.2.2 Let $S$ be a surface of genus $g \geq 2$. Then the intersection forms $i_{\rho}$ on $\mathrm{T}_{[\rho]} \mathcal{M}^{z d}(S, G) \cong H_{\rho}^{1}(\mathfrak{g})$ fit together to a symplectic form $\omega$ on $\mathcal{M}^{z d}(S, G)$.

Definition 6.2.3 The above symplectic form is called the Atiyah-Bott-Goldman symplectic form on the representation variety.

Now we prove our theorem:

Proof: [of Theorem 6.2.2] As we have already seen in Theorem 4.1.7 that the intersection forms are non-degenerate, we have only to show that $\omega$ is closed and smooth. 
It suffices to show that for any $\left[\rho_{0}\right] \in \mathcal{M}^{z d}(S, G)$ there exists a neighbourhood $U$ of $\left[\rho_{0}\right]$ such that $\left.\omega\right|_{U}$ is the pull-back of a smooth exact form. By Lemma 6.1.1 we may assume that there exists a bundle $E$ and a smooth map $f: U \rightarrow \mathcal{A}_{E}$ such that the holonomy representation of $f([\rho])$ is given by $[\rho]$. On the underlying vector space $A_{E}$ we have a pairing $\Omega$ given by

$$
\Omega(\alpha, \beta):=\int_{S} \operatorname{Tr}(\alpha \wedge \beta),
$$

where

$$
\operatorname{Tr}(\alpha \wedge \beta)(x, y)=\operatorname{Tr}_{\operatorname{End}(E)}(\alpha(x) \beta(y)-\beta(y) \alpha(x)) .
$$

As the tangent space of $\mathcal{A}_{E}$ at any point is identified with $A_{E}, \Omega$ induces a smooth 2-form on $\mathcal{A}_{E}$. If $\nabla_{0}$ is any fixed connection on $E$ and $\xi$ is the one-form on $A_{E}$ given by

$$
\xi_{\nabla}(\alpha)=\int_{S} \operatorname{Tr}\left(\left(\nabla-\nabla_{0}\right) \wedge \alpha\right),
$$

then $\Omega=\mathrm{d} \xi$. Thus $\Omega$ is a smooth exact 2-form on $\mathcal{A}_{E}$. From the definition of the intersection form we see that $\left.\omega\right|_{U}=f^{*} \Omega$ and the theorem follows. Q.E.D.

\subsection{Classical mechanics and observables}

In classical mechanics symplectic manifolds $(M, \omega)$ model phase spaces. In this context, elements of $C^{\infty}(M)$ are regarded as observables. The dynamics of the observables can be expressed in term of the Poisson bracket, which is defined as follows: using the symplectic form we can associate to every observable $f$ a vector field $H_{f}$ called the Hamiltonian vector field of $f$ and given by

$$
\mathrm{d} f(u)=\omega\left(u, H_{f}\right) .
$$

Then the Poisson bracket $\{\cdot, \cdot\}: C^{\infty}(M) \times C^{\infty}(M) \rightarrow C^{\infty}(M)$ is defined by

$$
\{f, g\}:=\omega\left(H_{f}, H_{g}\right)=\mathrm{d} g\left(H_{f}\right) .
$$

The set of observables has the following algebraic structure:

Proposition 6.3.1 If $(M, \omega)$ is a symplectic manifold the $\left(C^{\infty}(M),\{\cdot, \cdot\}, \cdot\right)$ is a Poisson algebra, in other words enjoys the following properties

- $\left(C^{\infty}(M),\{\cdot, \cdot\}\right)$ is a Lie algebra,

- $\left(C^{\infty}(M), \cdot\right)$ is a commutative associative algebra, 
- the two structures are compatible in the sense that Leibniz identity holds

$$
\{f, g h\}=h\{f, g\}+g\{f, h\} .
$$

Of course, it is rather naive to call any arbitrary function on $M$ an observable - at least from a physical point of view. Namely, one is usually only interested in observing some special quantities like energy, momentum etc. and not just arbitrary functions. Thus there should be a subset of $C^{\infty}(M)$ consisting of interesting observables. As the dynamics of the observable is governed by the Poisson algebra structure, a collection of interesting observables of $M$ should be a Poisson subalgebra of $C^{\infty}(M)$. In the case of the representation variety, there are various such subalgebras of geometric interest. In this subsection and the next we discuss two such subalgebras, which are related to the topology of the surface $S$ and feature prominently in topological quantum field theory.

\subsection{Special observables I: Wilson loops and the Goldman algebra}

We first recall some elementary facts about loops and conjugacy classes in the fundamental group. The free homotopy class of a loop is the homotopy class of the loop as a map from the circle to the surface $S$.

Choosing a base point $x$ in $S$ and path $\alpha$ from $x$ to a point in the loop $\gamma$, we obtain a loop $\eta=\alpha \cdot \gamma \alpha^{-1}$ based at $x$ and thus an element in $\pi_{1}(S, x)$. Different choices of the connecting path lead to conjugate elements in $\pi_{1}(S, x)$. More generally, a free homotopy class of loop defines a conjugacy class in $\pi_{1}(S, s)$. Conversely every conjugacy class in $\pi_{1}(S, x)$ defines a free homotopy class of loop. We denote by $[\gamma]$ the conjugacy class of $\gamma$ in $\pi_{1}(S, x)$, or equivalently the free homotopy class of the loop $\gamma$.

Note finally that if $\rho$ is a representation of $\pi_{1}(S, s)$ in $G$, the conjugacy class $[\rho(\gamma)]$ of $\rho(\gamma)$ in $G$ only depends on the conjugacy classes of $\gamma$ and $\rho$. This remark allows us the following definition since traces only depends on conjugacy classes.

Definition 6.4.1 [WILSON LOOPS] Let $[\gamma]$ be a conjugacy class in $\pi_{1}(S)$. Let $\lambda$ be a linear representation of $G$. The following function

$$
\mathbf{w}_{[\gamma]}: \mathcal{M}^{z d}(S, G) \rightarrow \mathbb{R}, \quad[\rho] \mapsto \operatorname{Tr}(\lambda[\rho]([\gamma])),
$$

is called, following physics literature, the Wilson loop associated to $\gamma$ and $\lambda$. 
Exercise 6.4.2 Let $\nabla$ be the flat connection associated to $\lambda$ and $\rho$. Let $[\gamma]$ be a homotopy class of loop. Check that

$$
\mathbf{w}_{[\gamma]}([\nabla])=\operatorname{Tr}\left(\operatorname{Hol}_{\nabla}(\gamma)\right) .
$$

In order to understand the dynamics of the Wilson loops it is important to understand the structure of the Poisson algebra that they generate in $C^{\infty}\left(\mathcal{M}^{z d}(S, G)\right)$. For this it is enough to understand the Poisson bracket of two Wilson loops, as the general case can be reduced to this using the axioms of a Poisson algebra. In order to carry out this computation, we introduce another closely related algebra, which can be defined in purely geometric terms.

Let $\mathcal{G}_{S}$ be the real vector space spanned by the free homotopy classes of loops, where the trivial loop is identified with zero.

We begin with a proposition. First we recall that if two oriented curves $c_{1}$ and $c_{2}$ in $S$ meet transversely at a point $x$, we define $\epsilon_{x}\left(\gamma_{1}, \gamma_{2}\right)$ to be 1 if $\left(\dot{c}_{1}, \dot{c}_{2}\right)$ is oriented and -1 otherwise. Then

Proposition 6.4.3 Let $\gamma_{1}, \gamma_{2}$ be two loops in $S$ with transversal intersections. Then, the following element of $\mathcal{G}_{S}$

$$
\left\{\gamma_{1}, \gamma_{2}\right\}:=\sum_{x \in \gamma_{1} \cap \gamma_{2}} \epsilon_{x}\left(\gamma_{1}, \gamma_{2}\right)\left[\gamma_{1} \sharp x \gamma_{2}\right]
$$

only depends on the classes $\left[\gamma_{1}\right],\left[\gamma_{2}\right]$. Moreover, if we define $\left\{\left[\gamma_{1}\right],\left[\gamma_{2}\right]\right\}:=$ $\left\{\gamma_{1}, \gamma_{2}\right\}$, then $\left(\mathcal{G}_{S},\{\},\right)$ is a Lie algebra.

This yields the following definition.

Definition 6.4.4 The Lie algebra $\left(\mathcal{G}_{S},\{\cdot, \cdot\}\right)$ is called the Goldman algebra.

The precise relation between the Goldman algebra and the Poisson algebra generated by the Wilson loops depends on the target group $G$. For example one has:

Theorem 6.4.5 [Goldman] Let $S$ be a surface, $G$ a linear Lie group and let $\left[\gamma_{1}\right],\left[\gamma_{2}\right]$ be two free homotopy classes of loops in $S$ with associated Wilson loops $\mathbf{w}_{\left[\gamma_{1}\right]}, \mathbf{w}_{\left[\gamma_{2}\right]} \in C^{\infty}\left(\mathcal{M}^{z d}(S, G)\right)$.

(i) If $G=\mathrm{GL}(n, \mathbb{R})$ then

$$
\left\{\mathbf{w}_{\left[\gamma_{1}\right]}, \mathbf{w}_{\left[\gamma_{2}\right]}\right\}=\mathbf{w}_{\left\{\left[\gamma_{1}\right],\left[\gamma_{2}\right]\right\}}
$$


(ii) If $G=\mathrm{SL}(n, \mathbb{R})$ then

$$
\left\{\mathbf{w}_{\left[\gamma_{1}\right]}, \mathbf{w}_{\left[\gamma_{2}\right]}\right\}=\mathbf{w}_{\left\{\left[\gamma_{1}\right],\left[\gamma_{2}\right]\right\}}-\frac{1}{n} i\left(\left[\gamma_{1}\right],\left[\gamma_{2}\right]\right) \mathbf{w}_{\left[\gamma_{1}\right]} \mathbf{w}_{\left[\gamma_{2}\right]},
$$

where $i\left(\left[\gamma_{1}\right],\left[\gamma_{2}\right]\right)=\sum_{x \in \gamma_{1}, \gamma_{2}} \epsilon_{x}\left(\gamma_{1}, \gamma_{2}\right)$ denotes the algebraic intersection number of $\gamma_{1}$ and $\gamma_{2}$ which only depends on the homotopy classes of $\left[\gamma_{1}\right]$ and $\left[\gamma_{2}\right]$.

Actually

\section{Hamiltonian vector field of Wilson loops}

Our first step in the proof Theorem 6.4.5 is to compute the Hamiltonian vector field of a Wilson loop. Thus let us fix a loop $\gamma$ and denote by $H:=H_{\mathbf{w}_{[\gamma]}}$ the Hamiltonian vector field associated to the corresponding Wilson loop $\mathbf{w}_{[\gamma]}$.

We identify $\mathrm{T}_{[\rho]} \mathcal{M}^{z d}(S, G)$ for $[\rho]$ in $\mathcal{M}^{z d}(S, G)$ with $H_{\rho}^{1}(\mathfrak{g})$. Recall from Definition 4.1.10 that this $H_{\rho}^{1}(\mathfrak{g})$ is the twisted cohomology of a certain bundle with connection $\left(\mathcal{L}_{\mathfrak{g}}, \nabla_{\mathfrak{g}}\right)$ whose typical fibre is the Lie algebra $\mathfrak{g}$ of $G$.

Thus $H$ is represented by a class of closed 1-forms $H_{\nabla}$ on $S$ with values in the bundle $\mathcal{L}_{\mathfrak{g}}$. We are going to construct such a 1-form explicitly.

We assume that $G \subset \mathrm{GL}(n, \mathbb{R})$ is reductive - as are our classical examples $\mathrm{SU}(n), \operatorname{SL}(n, \mathbb{R})$. On $M_{n}(\mathbb{R})$ we have a non-degenerate inner product given by $(A, B) \mapsto \operatorname{Tr}(A, B)$. The reductivity assumption translates in the fact that $M_{n}(\mathbb{R})$ splits as

$$
M_{n}(\mathbb{R})=\mathfrak{g} \oplus \mathfrak{g}^{\perp}
$$

From the point of view of bundle, we consider an associated $G$-bundle $E$ of rank $n$ equipped with a flat connection, so that $\left(\mathcal{L}_{\mathfrak{g}}, \nabla_{\mathfrak{g}}\right)$ is a subbundle of the bundle $\operatorname{End}(E)$. The above splitting translates in a parallel orthogonal splitting

$$
\operatorname{End}(E)=\mathcal{L}_{\mathfrak{g}} \oplus \mathcal{L}_{\mathfrak{g}}^{\perp}
$$

Denote by $X \mapsto \widehat{X}$ the projection onto the first summand.

In order to simplify things, let us assume that $\gamma$ is does not have any selfintersection.

We describe the step of the construction and first make the following choices. Let $U$ be a tubular neighbourhood of $\gamma$ and $\beta_{\gamma}$ a closed 1-form for with support in $U$ so that for any closed 1-form $\alpha$ defined on $U$ and for any transverse curve $c$ to $\gamma$

$$
\begin{aligned}
\int_{U} \alpha \wedge \beta_{\gamma} & =\int_{\gamma} \alpha, \\
\int_{c} \beta_{\gamma} & =i(c, x) .
\end{aligned}
$$


Such a form $\beta_{\gamma}$ is easily constructed out of the derivative of a bump function.

For any point $x \in U$, the holonomy $\operatorname{Hol}_{\gamma}(x)$ of a loop based at $x$ and freely homotopic to $\gamma$ in $U$ with respect to the connection $\nabla=\nabla_{\mathfrak{g}}$ is an automorphism of the fibre $E_{x}$. Observe that $\mathrm{Hol}_{\gamma}: U \rightarrow \operatorname{End}(E)$ is a well defined section which is furthermore parallel ( $c f$ Exercise 3.3.11).

By a slight abuse of language we denote by $\widehat{\mathrm{Hol}}_{\gamma} \cdot \beta_{\gamma}$ the 1 -form, with values in $\mathcal{L}_{\mathfrak{g}}$ which vanishes outside $U$ and is equal to $\widehat{\mathrm{Hol}}_{\gamma} \cdot \beta_{\gamma}$, on $U$. One easily checks that this form is closed.

We claim that the cohomology class of this form is the symplectic gradient of the Wilson loop.

Proposition 6.4.6 Let notation be as above. Then the class of $\widehat{\mathrm{Hol}}_{\gamma} \cdot \beta_{\gamma}$ in $H_{\rho}^{1}(\mathfrak{g})$ represents $H_{\nabla}$.

Proof: We choose a family of $\nabla_{t}$ a family of flat connections deforming $\nabla=\nabla_{0}$. We denote by $[\dot{\nabla}]$ the tangent vector $t=0$ to the curve in $\mathcal{M}^{z d}(S, G)$ given by $t \rightarrow\left[\nabla_{t}\right]$. We have a representative of $[\dot{\nabla}]$ given by

$$
\dot{\nabla}=\left.\frac{\mathrm{d}}{\mathrm{d} t}\right|_{t=0}\left(\nabla_{t}-\nabla_{0}\right) \in \Omega^{1}\left(S, \mathcal{L}_{\mathfrak{g}}\right) .
$$

We want to compute

$$
\left.\frac{\mathrm{d}}{\mathrm{d} t}\right|_{t=0} \operatorname{tr}\left(\operatorname{Hol}_{\nabla_{\mathrm{t}}}(\gamma)\right) .
$$

We consider $\gamma$ as a $\mathbb{Z}$ periodic map from $\mathbb{R}$ to $S$. We choose a trivialisation of $\gamma^{*} E$ so that $\gamma^{*} \nabla_{0}$ is the trivial connection $\mathrm{D}$ and write $\gamma^{*} \nabla_{t}=\mathrm{D}+A_{t}$. Let $\frac{\partial}{\partial s}$ be the canonical vector field on $\mathbb{R}$ and

$$
B_{t}(s):=A_{t}\left(\frac{\partial}{\partial s}\right) .
$$

Then by Proposition 3.3.12 the holonomy of $\nabla_{t}$ is obtained by solving the following linear ordinary differential equation

$$
(*) \quad \frac{\mathrm{d} X}{\mathrm{~d} s}=B_{t} X
$$

More precisely, recall that the resolvent $u, s \rightarrow{ }^{t} R_{u}^{s}$ of this equation is by definition such that $X(s):={ }^{t} R_{u}^{s} X(u)$ is the solution of $(*)$ with initial condition $X(u)$ at time $u$. Then,

$$
\operatorname{Hol}_{\nabla_{t}}(\gamma)={ }^{t} R_{0}^{1}
$$


The method of the variation of constants yields

$$
\left.\frac{\mathrm{d}}{\mathrm{d} t}\right|_{t=0} \operatorname{Tr}\left(\operatorname{Hol}_{\nabla_{t}}(\gamma)\right)=\left.\frac{\mathrm{d}}{\mathrm{d} t}\right|_{t=0} \operatorname{Tr}\left({ }^{t} R_{0}^{1}\right)=\int_{0}^{1} \operatorname{Tr}\left({ }^{t} R_{s}^{1+s} \dot{B}(s)\right) \mathrm{d} s,
$$

where

$$
\dot{B}=\left.\frac{\mathrm{d}}{\mathrm{d} t}\right|_{t=0} B_{t}(s)=\dot{\nabla}\left(\frac{\partial f}{\partial s}\right) .
$$

We now interpret the right-hand side term and rewrite this integral as

$$
\begin{aligned}
\int_{0}^{1} \operatorname{Tr}\left({ }^{0} R_{s}^{1+s} \dot{B}(s)\right) \mathrm{d} s & \left.=\int_{0}^{1} \operatorname{Tr}\left(\operatorname{Hol}_{\nabla_{0}}(\gamma)_{\gamma(s)}\right) \dot{\nabla}\left(\frac{\partial \gamma}{\partial s}\right)\right) \mathrm{d} s \\
& =\int_{\gamma} \operatorname{Tr}\left(\operatorname{Hol}_{\gamma} \cdot \dot{\nabla}\right) \\
& =\int_{\gamma} \operatorname{Tr}\left(\widehat{\operatorname{Hol}_{\gamma}} \cdot \dot{\nabla}\right)
\end{aligned}
$$

In the last equality, we have used that $\operatorname{Tr}(a u)=\operatorname{Tr}(\hat{a} u)$ if $u$ belongs to $\mathfrak{g}$. Let $\alpha$ be the 1-form defined on $U$ by

$$
\alpha=\operatorname{Tr}\left(\widehat{\operatorname{Hol}_{\gamma}} \cdot \dot{\nabla}\right) .
$$

Note that $\alpha$ is closed. It follows by Equation (6.3), that

$$
\int_{U} \alpha \wedge \beta_{\gamma}=\int_{\gamma} \alpha
$$

Therefore combining the previous equations, we obtain that

$$
\begin{aligned}
\left.\frac{d}{d t}\right|_{t=0} \operatorname{Tr}\left(\operatorname{Hol}_{\nabla_{t}}(\gamma)\right. & =\int_{\gamma} \alpha \\
& =\int_{U} \alpha \wedge \beta_{\gamma} \\
& =\int_{U} \operatorname{Tr}\left(\widehat{\operatorname{Hol}_{\gamma}} \dot{\nabla}\right) \wedge \beta_{\gamma} \\
& =-\int_{S} \operatorname{Tr}\left(\widehat{\mathrm{Hol}_{\gamma}} \cdot \beta_{\gamma} \wedge \dot{\nabla}\right) .
\end{aligned}
$$

Using the symplectic form $\omega$ on $\mathcal{M}^{z d}(S, G)$ we finally obtain

$$
d \mathbf{w}_{\gamma}(\rho)=-\int_{S} \operatorname{Tr}\left(\widehat{\operatorname{Hol}}_{\gamma} \cdot \beta_{\gamma} \wedge \dot{\nabla}\right)=-\omega\left(\widehat{\operatorname{Hol}}_{\gamma} \cdot \beta_{\gamma}, \dot{\nabla}\right)
$$

Thus by definition

$$
H_{\nabla_{0}}=\left[\widehat{\operatorname{Hol}}_{\gamma} \cdot \beta_{\gamma}\right]
$$

Q.E.D. 
Exercise 6.4.7 Deduce Theorem 6.4.5 from Proposition 6.4.6.

\subsection{Special observables II: Spin networks}

A larger class of observables on $\mathcal{M}^{z d}(S, G)$ which includes Wilson loops 6.4.1 as a special case can be constructed using embeddings of so-called spin networks. The starting definition is the following:

Definition 6.5.1 [SPIN NETwORKS] $A$ spin network $\mathfrak{S}$ for $G$ consists of the following data

- an oriented graph $\Gamma=\Gamma^{\mathfrak{S}}=(V, E)$ called the underlying graph of $\mathfrak{S}$.

- for every $e \in E$, a vector space $I_{e}=I_{e}^{\mathfrak{S}}$ and a representation $\lambda_{e}=\lambda_{e}^{\mathfrak{S}}$ : $G \rightarrow \operatorname{Aut}\left(I_{e}\right)$.

- for any $v \in V$, a linear map

$$
i_{v}=i_{v}^{\mathfrak{S}} \in \operatorname{Hom}\left(\bigotimes_{\left\{e \in E \mid e_{+}=v\right\}} I_{e}, \bigotimes_{\left\{e \in E \mid e_{-}=v\right\}} I_{e}\right)
$$

which intertwines the $G$-actions in the sense that for any $g \in G$,

$$
i_{v} \circ\left(\bigotimes_{\left\{e \in E \mid e_{+}=v\right\}} \lambda_{e}(g)\right)=\left(\bigotimes_{\left\{e \in E \mid e_{-}=v\right\}} \lambda_{e}(g)\right) \circ i_{v}
$$

We observe that the group $G^{E}$ naturally acts on the space of spin networks with a common underlying graph. Namely, for every edge $e$ we conjugate the representation by $g(e)$, and for every vertex we perform the coherent transformation on the intertwiner. This action is called the gauge action on spin networks.

Definition 6.5.2 [EMBEDDING OF A SPIN NETWORK] Let $S$ be a surface equipped with a bundle $\mathcal{L}$. An embedding of the spin network $\mathfrak{S}$ into $S$ is by definition:

- an embedding of $\Gamma^{\mathfrak{S}}$ into $S$,

- for every vertex $v$ in the underlying graph, an identification of $\mathcal{L}_{\Psi(v)}$ with a fixed vector space $L$. 
We say two embeddings of to spin networks equivalent under the gauge action are isotopic embeddings of spin networks if the corresponding embedding of the underlying graph are isotopic. For instance, fixing the embedding of the graph, and changing the identification of the fibres at images of vertices with L, yield isotopic embedding of spin network. An isotopy class is called an embedded spin network.

\subsubsection{An observable on the space of all flat connections}

Now, we shall define a function on the space of flat connections on $\mathcal{L}$ associated to every embedding of a spin network in $S$.

Let $\mathfrak{S}$ be a spin network and let $\Psi: \mathfrak{S} \rightarrow S$ be an embedding into some surface $S$. Let $\nabla$ be a flat connection on some bundle $\mathcal{L}$ with fibre $L$. Using the identification of $\mathcal{L}_{\Psi(v)}$ with $L$, for every edge $e$, holonomy $H(e):=H_{\nabla o l}(\Psi(e))$ becomes an element of $G$. Thus $\lambda_{e}(H(e)) \in \operatorname{End}\left(I_{e}\right)$. We now define

$$
\Lambda_{\Psi}(\nabla):=\bigotimes_{e \in E} \lambda_{e}(H(e)) \in \operatorname{Aut}\left(\bigotimes_{e \in E} I_{e}\right) \text {. }
$$

On the other hand, we have

$$
\begin{aligned}
I_{\Psi}:=\bigotimes_{v \in V} i_{v}^{\mathfrak{S}} & \in \bigotimes_{v \in V}\left(\operatorname{Hom}\left(\bigotimes_{\left\{e \in E \mid e_{+}=v\right\}} I_{e}, \bigotimes_{\left\{e \in E \mid e_{-}=v\right\}} I_{e}\right)\right) \\
& =\operatorname{Hom}\left(\bigotimes_{v \in V} \bigotimes_{\left\{e \in E \mid e_{+}=v\right\}} I_{e}, \bigotimes_{v \in V} \bigotimes_{\left\{e \in E \mid e_{-}=v\right\}} I_{e}\right) \\
& =\operatorname{End}\left(\bigotimes_{e \in E} I_{e}\right)
\end{aligned}
$$

Then we can multiply

$$
\Lambda_{\Psi}(\nabla) \cdot I_{\Psi} \in \text { End }\left(\bigotimes_{e \in E} I_{e}\right),
$$

Thus, we define an observable on the space of all flat connections by

$$
f_{\Psi}: \nabla \mapsto \operatorname{Tr}\left(\Lambda_{\Psi}(\nabla) \cdot I_{\Psi}\right) .
$$

Definition 6.5.3 [SPIN NETWORK OBSERVABLE] The function $f_{\Psi}$ described above is called the spin network observable to associated to $\Psi$.

\section{REMARK:}

1. Observe that this construction extends the notion of a Wilson loop as a loop is nothing but an embedding of the trivial spin network whose underlying graph has one vertex and one edge and whose only intertwiner $i_{v}$ is the identity. 
An observable on $\mathcal{M}^{z d}(S, G)$

The following proposition says that the spin network is a well defined observable on $M$

Proposition 6.5.4 [INVARIANCE OF THE SPIN NETWORK] The value of $f_{\Psi}$ at $\nabla$ is invariant under gauge action on $\nabla$, and isotopies of the embedding of the spin network. Hence, it yields a function $f_{\Psi}$ on $\mathcal{M}^{z d}(S, G)$

Definition 6.5.5 [SPIN NETWORK OBSERVABLE (BIS)] By a new abuse of language, we call again the corresponding function a spin network observable.

\subsubsection{Poisson bracket of spin network observables}

We define the space $\mathfrak{S}$ of spin networks on $S$ as the $\mathbb{R}$-module generated by the equivalence classes of spin networks - actually it should be a quotient of that space .... By the above definition, the map $\Psi \mapsto f_{\psi}$ extend to a linear map

$$
\text { ev }: \mathfrak{S} \rightarrow C^{\infty}\left(\mathcal{M}^{z d}(S, G)\right)
$$

We will now define a Lie bracket on $\mathfrak{S}$ which generalises the Poisson bracket on the Goldman algebra. For this let $\mathcal{S}_{1}, \mathfrak{S}_{2}$ be two spin networks and let

$$
\begin{aligned}
& \Psi_{1}: \mathfrak{S}_{1} \rightarrow S \\
& \Psi_{2}: \mathfrak{S}_{2} \rightarrow S
\end{aligned}
$$

be two embeddings. Assume that the images of the underlying oriented graphs $\Gamma_{1}, \Gamma_{2}$ intersect transversely. As in the case of the Goldman algebra we shall define for each intersection point $x \in \Gamma_{1} \cap \Gamma_{2}$ a sign $\epsilon_{x}$ and new spin network $\mathfrak{S}_{1} \sharp_{x} \mathfrak{S}_{2}$ defined below as well as an embedding $\Psi_{x}$ of this spin network. We finally define

$$
\left[\Psi_{1}, \Psi_{2}\right]:=\sum_{x \in \Gamma_{1} \cap \Gamma_{2}} \epsilon_{x} \cdot \Psi_{x}
$$

Let us make this definition precise.

- The definition of $\epsilon_{x}$ is as in the case of the Goldman algebra: $\epsilon_{x}$ is 1 if the orientations of $\Gamma_{1}$ and $\Gamma_{2}$ are such that $\Gamma_{2}$ crosses $\Gamma_{1}$ at $x$ from right to left and -1 otherwise.

- The underlying graph of $\left(\mathfrak{S}_{1} \sharp_{x} \mathfrak{S}_{2}, \Psi_{x}\right)$ does not depend on $x$ and is just a transverse superposition of the underlying graphs of $\mathfrak{S}_{1}$ and $\mathfrak{S}_{2}$ : its vertices are the union of the vertices of $\Gamma_{1}$ and $\Gamma_{2}$ and all intersection points of $\Gamma_{1}$ and $\Gamma_{2}$, its edges are connected components of

$$
\mathfrak{S}_{1} \cup \mathfrak{S}_{2} \backslash\left(\mathfrak{S}_{1} \cap \mathfrak{S}_{2}\right)
$$


and the embedding is the one given by $\Psi_{1}$ and $\Psi_{2}$.

- Any edge is a subedge of an edge of either $\Gamma_{1}$ or $\Gamma_{2}$ and it is labelled by the corresponding $G$-module.

- The vertices of $\Gamma_{1}$ and $\Gamma_{2}$ are labelled by the same intertwiners as before.

- Finally, suppose $y$ is an intersection point of an edge $e_{1}$ of $\Gamma_{1}$ with an edge $e_{2}$ of $\Gamma_{2}$. Let $\left(I_{1}, \lambda_{1}\right),\left(I_{2}, \lambda_{2}\right)$ be the corresponding $G$-modules. Then we have to define an intertwiner $i_{y}: I_{1} \otimes I_{2} \rightarrow I_{2} \otimes I_{1}$. Such intertwiners can be constructed using the Lie algebra representations $\mathrm{D} \lambda_{j}: \mathfrak{g} \rightarrow \operatorname{End}\left(I_{j}\right)$, $j=1,2$ : Assume that there exists a non-degenerate invariant bilinear form $\langle\cdot, \cdot\rangle$ on $\mathfrak{g}$. For a simple Lie algebra we can take the Killing form, but such a form may also exist for non-simple Lie algebras, for example $\mathfrak{g l}(n, \mathbb{R})$. Let $e_{1}, \ldots, e_{k}$ be a basis of $\mathfrak{g}$ which is orthonormal with respect to $\langle\cdot, \cdot\rangle$. Then one checks that the Casimir intertwiner

$$
\triangle:=\sum_{i=1}^{k} \mathrm{D} \lambda_{1}\left(e_{i}\right) \otimes \mathrm{D} \lambda_{2}\left(e_{i}\right) \in \operatorname{End}\left(I_{1} \otimes I_{2}\right)
$$

is independent of the choice of orthonormal basis and intertwines the tensor product action of $G$ on $I_{1} \otimes I_{2}$ with itself. Thus we define

$$
i_{y}:=\left\{\begin{array}{ccc}
\triangle, & \text { if } & y=x \\
\mathrm{Id}, & \text { if } & y \neq x
\end{array}\right.
$$

This finishes the construction of $\left(\mathfrak{S}_{1} \sharp_{x} \mathfrak{S}_{2}, \Psi_{x}\right)$. As in the case of Wilson loops the Poisson bracket of spin network observables is closely related to the Lie bracket of embedded spin networks. In the case of $\operatorname{GL}(n, \mathbb{R})$ one has:

Theorem 6.5.6 Let $G=\operatorname{GL}(n, \mathbb{R})$ and let $\Psi_{1}, \Psi_{2}$ be embedded spin networks in a surface $S$. Then the associated spin network observables $f_{\Psi_{1}}, f_{\Psi_{2}} \in$ $C^{\infty}\left(\mathcal{M}^{z d}(S, G)\right)$ satisfy

$$
\left\{f_{\Psi_{1}}, f_{\Psi_{2}}\right\}=f_{\left[\Psi_{1}, \Psi_{2}\right]}
$$

We leave this theorem as a stimulating exercise for the reader. In particular one gets

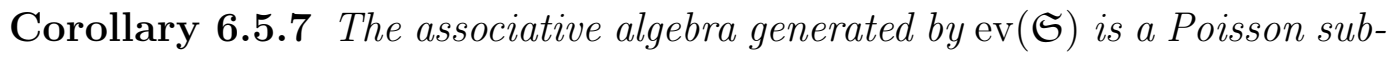
algebra. 


\subsection{Volumes of moduli spaces}

In this section, we compute the symplectic volume of the moduli space when $G$ is compact. This proof due to Witten [Wit91] and will make use of the main result of our chapter on torsion which identifies the torsion and the symplectic volume on cohomology groups.

\subsubsection{Witten's formula}

Recall that the very regular part carries a symplectic form.

Theorem 6.6.1 [WiTTEN'S FORMULA] Let $S$ be a surface of genus $g$ and let $G$ be a simply-connected simple compact Lie group with finite centre $Z(G)$. Then the volume of the moduli space $\mathcal{M}(S, G)$ with respect to the symplectic form is given by

$$
\operatorname{vol}(\mathcal{M}(S, G))=|Z(G)| \sum_{\lambda \in \widehat{G}}\left(\frac{\operatorname{vol}(G)}{\operatorname{dim} V_{\lambda}}\right)^{2 g-2},
$$

where the volume of $G$ is computed with respect to the Killing form, $\widehat{G}$ is the the set of finite dimensional representations $\lambda$ of $G$ and $V_{\lambda}$ is the vector space associated to $\lambda$.

\section{REMARKS:}

1. [ON NORMALisAtion] Given a symplectic manifold $(M, \omega)$ of dimension $2 d$, the associated volume form is the Liouville volume $\frac{1}{d !} \omega^{d}$.

2. In this theorem, everything - the symplectic form and the volume of the group - is computed with respect to the Killing form $k$. We could have chosen another normalisation. Let just see what happens when we choose instead of $k$, the metric $\alpha k$. Then

$$
\operatorname{vol}_{\alpha}(G)=\alpha^{\frac{\operatorname{dim} G}{2}} \operatorname{vol}(G)
$$

and

$$
\operatorname{vol}_{\alpha}(\mathcal{M}(S, G))=\alpha^{(g-1) \operatorname{dim} G} \operatorname{vol}(\mathcal{M}(S, G)) .
$$

Hence the formula is homogeneous.

For reason that are going to be clearer in the next section, it is also quite natural to choose $\frac{1}{4 \pi^{2}} k$ as it is done in Witten's' original paper. This normalisation is motivated by the fact that $\frac{1}{4 \pi^{2}} \omega$ is integral (as we shall 
see below) and is used e.g. by Witten. Therefore the original Witten formula reads as

$$
\operatorname{vol}^{*}\left(\mathcal{M}^{z d}(S, G)\right)=\left(\frac{1}{2 \pi}\right)^{-\chi(S) \operatorname{dim} G} \sum_{\lambda \in \widehat{G}}|Z(G)| \cdot \frac{(\operatorname{vol}(G))^{2 g-2}}{\left(\operatorname{dim} V_{\lambda}\right)^{2 g-2}},
$$

which differs from our formula precisely by the factor $\left(\frac{1}{2 \pi}\right)^{-\chi(S)} \operatorname{dim} G$.

3. For $G=\mathrm{SU}(2)$, we have $\widehat{G} \cong \mathbb{N}$ and $\operatorname{dim} V_{n}=n$. Moreover, $Z(\mathrm{SU}(2))=$ $\{ \pm 1\}$. The volume of $G$ with respect to the Killing form is $2^{\frac{5}{2}} \pi^{2}$. Thus we obtain

$$
\operatorname{vol}(S, \mathcal{M}(\mathrm{SU}(2)))=2\left(2^{(5 g-5)} \pi^{4 g-4}\right) \sum_{n=1}^{\infty} n^{-(2 g-2)} .
$$

We rather choose the metric $\frac{1}{4 \pi^{2}} k$. Hence we get,

$$
\operatorname{vol}_{\frac{1}{4 \pi^{2}}}(\mathcal{M}(S, G))=2 \frac{\left(2^{(5 g-5)} \pi^{4 g-4}\right)}{2^{6 g-6} \pi^{6 g-6}} \sum_{n=1}^{\infty} n^{-(2 g-2)}=\frac{\zeta(2 g-2)}{2^{g-2} \pi^{2 g-2}}
$$

where $\zeta(z)$ denotes the Riemann zeta function. In particular, the volume with respect to this normalisation is rational.

The proof goes in two steps. The first step is to identify this measure with a measure obtained by disintegration in Proposition 6.6.3. And the second step is to compute by induction in Proposition 6.6.6 the volume with respect to this measure.

\subsubsection{Volume and the disintegrated measure}

We first recall the definition of a disintegrated measure. In a presence of a map between two measured spaces, there is a general way to construct a family of measures on the fibres.

Proposition 6.6.2 Let $\left(X, \nu_{X}\right)$ and $\left(Y, \nu_{Y}\right)$ be finite measure spaces and let $f: X \rightarrow Y$ be a measurable map, such that $f_{*} \nu_{X}$ is absolutely continuous with respect to $\nu_{Y}$, then there exists a unique measurable family of measures $\left\{\nu_{y}\right\}_{y \in Y}$, where $\nu_{y}$ is a measure on $f^{-1}(y)$, such that for all measurable $g: X \rightarrow \mathbb{R}$ the identity

$$
\int_{X} g(x) \mathrm{d} \nu_{X}(x)=\int_{Y}\left(\int_{f^{-1}(y)} g(x) \mathrm{d} \nu_{y}(x)\right) \mathrm{d} \nu_{Y}(y) .
$$

holds. 
The family $\left\{\nu_{y}\right\}_{y \in Y}$ is said to be obtained by disintegration of $\left(X, \nu_{X}\right)$ and $\left(Y, \nu_{Y}\right)$ with respect to $f$.

In the next proof we will not use the full force of this result and just prove and use it in a geometric differential context.

We now interpret the symplectic volume using a disintegrated measure Let $\mu: G^{2 g} \rightarrow G$ be the map given by

$$
\mu\left(a_{1}, b_{1}, \ldots, a_{g}, b_{g}\right)=\prod_{i=1}^{i=g}\left[a_{i}, b_{i}\right] .
$$

We equip $G$ with the Killing form.

Theorem 6.6.3 Let $\nu$ be the disintegrated measure on $\mu^{-1}$. Then

$$
\int_{\mu^{-1}(\mathrm{Id})} \nu=\frac{|Z(G)|}{\operatorname{vol}(G)} \int_{\mathcal{M}(S, G)} \frac{\omega^{n}}{n !},
$$

where $n=\frac{1}{2} \operatorname{dim}(\mathcal{M}(S, G))$ and $\omega$ is the symplectic form on $\mathcal{M}(S, G)$.

\section{Preliminaries}

We first need to understand in a geometric differential way disintegration of measures. This is the purpose of the next lemma.

Lemma 6.6.4 We use the same notation as in Proposition 6.6.2. When $X$ and $Y$ are smooth, $f$ is a submersion, and $\nu_{X}$ and $\nu_{Y}$ come from volume forms $\Omega_{X}$ and $\Omega_{Y}$, the measure $\nu_{y}$ also comes from a volume form $\Omega_{y}$. This family of measures satisfy

$$
\Omega_{X}=\Omega_{y} \otimes \Omega_{Y}
$$

where we use the identification

$$
\operatorname{det}\left(\mathrm{T}_{x} X\right)=\operatorname{det}\left(\operatorname{ker} \mathrm{T}_{x} f\right) \otimes \operatorname{det}\left(\mathrm{T}_{f(x)} Y\right) .
$$

As a hint of the proof, let us just say that this is a local result and thus it suffices to consider the case where $X$ is a product and $f$ is a projection. The same proof gives the following proposition

Lemma 6.6.5 Let $K$ be a compact Lie group equipped with a biinvariant measure realised by a volume form $\Omega_{K}$. Let $M$ be a smooth manifold on which $K$ acts freely, equipped with a $K$-invariant volume form $\Omega_{M}$. Then there exists a unique volume form $\Omega_{M / K}$ on $M / K$ characterised by

$$
\Omega_{M}=\Omega_{M / K} \otimes \Omega_{K},
$$


when we use the exact sequence

$$
0 \rightarrow \mathfrak{K} \rightarrow \mathrm{T} M \rightarrow \mathrm{T}(M / K) \rightarrow 0,
$$

where the first arrow is the orbital map and the second arrow is the projection. Moreover

$$
\operatorname{vol}(M / K)=\frac{\operatorname{vol}(M)}{\operatorname{vol}(K)}
$$

\section{Proof of Theorem 6.6.3}

We saw in Paragraph 5.3.5 that for any representation in the very regular part the sequence

$$
0 \rightarrow \mathrm{T} G \rightarrow \mathrm{T} G^{2 g} \stackrel{\mathrm{T} \mu}{\rightarrow} \mathrm{T} G \rightarrow 0,
$$

corresponds term by term with the complex

$$
0 \rightarrow C^{0}\left(\Gamma_{g}\right) \rightarrow C^{1}\left(\Gamma_{g}\right) \rightarrow C^{2}\left(\Gamma_{g}\right) \rightarrow 0 .
$$

From the construction of Lemmas 6.6.4 and 6.6.5 and the definition 4.3.4 of the torsion, it follows that,

$$
\lambda=\operatorname{Tor}_{h}(\mathcal{G})
$$

where $h$ is the Killing form. From Theorem 4.3.8, it follows that

$$
\lambda=\frac{\omega^{\operatorname{dim} G(g-1)}}{\operatorname{dim} G(g-1) !},
$$

since $\operatorname{dim}\left(H_{\nabla}^{1}(\mathcal{L})\right)=(2 g-2)(\operatorname{dim} G)$.

Therefore, we have

$$
\int_{\mu_{z d}^{-1}(\mathrm{Id})} \nu=\frac{|Z(G)|}{\operatorname{vol}(G)} \int_{\mathcal{M}(S, G)} \frac{\omega^{n}}{n !},
$$

where $\mu_{z d}^{-1}(\mathrm{Id})$ is the intersection of $\mu^{-1}(\mathrm{Id})$ with the set of homomorphisms of Zariski dense image.

Finally, a closer inspection of the structure of the stratification of $\mu^{-1}(\mathrm{Id})$ is a reunion of smooth manifolds of dimension strictly less than that of $\mu^{-1}(\mathrm{Id})$ - see Lemma 5.3.6 and have measure zero with respect to the disintegrated measure. It follows that

$$
\int_{\mu_{z d}^{-1}(\mathrm{Id})} \nu=\int_{\mu^{-1}(\mathrm{Id})} \nu
$$

and the equality finishes the proof of Theorem 6.6.3 


\subsubsection{Characters and the disintegrated measure}

We now move to the last step needed in order to prove Witten's formula

Proposition 6.6.6 Let $S$ be a surface of genus $g$ and let $G$ be a simplyconnected simple compact Lie group with finite centre $Z(G)$. Then

$$
\int_{\mu^{-1}(\mathrm{Id})} \nu=\sum_{\lambda \in \widehat{G}} \frac{(\operatorname{vol}(G))^{2 g-1}}{\left(\operatorname{dim} V_{\lambda}\right)^{2 g-2}},
$$

where $\mu: G^{2 g} \rightarrow G$ is the map given by

$$
\mu\left(a_{1}, b_{1}, \ldots, a_{g}, b_{g}\right)=\prod_{i=1}^{i=g}\left[a_{i}, b_{i}\right] .
$$

In order to prove this proposition, we need properties of characters of $G$.

\section{Characters}

Let $\widehat{G}$ be the set of finite dimensional representations of $G$. Let $\lambda: G \rightarrow$ $\operatorname{GL}\left(V_{\lambda}\right)$ be an element of $\widehat{G}$. Such a representation is uniquely determined by its character $\chi_{\lambda}: G \rightarrow \mathbb{C}$, which is defined by $\chi_{\lambda}(h):=\operatorname{Tr} \lambda(h)$. Note that $\chi_{\lambda}(1)$ is the dimension of the module $V_{\lambda}$.

The characters $\chi_{\lambda}, \lambda \in \widehat{G}$ form an orthogonal basis of the subspace of $L^{2}(G)$ spanned by the conjugation invariant function. The following summarises the properties of characters as functions in $L^{2}$ that we shall need. Proofs can be found in Bröcker-tom Dieck [BtD85].

Proposition 6.6.7 Let $\lambda, \lambda^{\prime} \in \widehat{G}$. Then

$$
\begin{aligned}
\int_{G} \chi_{\lambda}(a g) \chi_{\lambda^{\prime}}\left(g^{-1} b\right) & =\frac{\operatorname{vol}(G)}{\chi_{\lambda}(1)} \chi_{\lambda}(a b) \delta_{\lambda, \lambda^{\prime}} . \\
\int_{G} \chi_{\lambda}\left(a h b h^{-1}\right) \mathrm{d} h & =\frac{\operatorname{vol}(G)}{\chi_{\lambda}(1)} \chi_{\lambda}(a) \chi_{\lambda}(b),
\end{aligned}
$$

where $\delta_{\lambda, \lambda^{\prime}}$ is the Kronecker symbol. Moreover if $f \in L^{2}(G)$ is invariant under conjugation then

$$
f(g)=\sum_{\lambda \in \widehat{G}} \frac{\chi_{\lambda}(g)}{\operatorname{vol}(G)} \int_{G} f(h) \chi_{\lambda}\left(h^{-1}\right) d h .
$$

REFERENCE in Bismut-Labourie

Using these properties of characters we can now deduce the main result of this section: 


\section{Proof of Proposition 6.6.6}

Let

$$
F(g)=\int_{\mu^{-1}(h)} \mathrm{d} \nu_{h}
$$

where $\left\{\nu_{h}\right\}_{h \in G}$ the family of measures obtained by disintegration along $\mu$. One checks that this is a conjugation invariant $L^{2}$-function on $G$ and thus Proposition 6.10 yields

$$
F(1)=\sum_{\lambda \in \widehat{G}} \frac{\chi_{\lambda}(1)}{\operatorname{vol}(G)} \int_{G} F(h) \chi_{\lambda}\left(h^{-1}\right) \mathrm{d} h .
$$

Thus by Proposition 6.6.2

$$
\begin{aligned}
\int_{G} F(h) \chi_{\lambda}\left(h^{-1}\right) d h & =\int_{G}\left(\int_{\mu^{-1}(h)}\left(\chi_{\lambda} \circ \mu\right) \mathrm{d} \lambda_{h}\right) \mathrm{d} \lambda_{G}(h) \\
& =\int_{G^{2 g}} \chi_{\lambda}\left(\prod_{i=1}^{i=g}\left[a_{i}, b_{i}\right]\right) d a_{1} \mathrm{~d} b_{1} \ldots d a_{g} \mathrm{~d} b_{g} .
\end{aligned}
$$

It remains to compute the integrals on the right hand side. Using Proposition 6.9 , we obtain

$$
\begin{aligned}
\int_{G} \chi_{\lambda}\left(\prod_{i=1}^{i=g}\left[a_{i}, b_{i}\right]\right) \mathrm{d} a_{1} & =\int_{G} \chi_{\lambda}\left(a_{1} b_{1} a_{1}^{-1} b_{1}^{-1} \cdot \prod_{i=2}^{g}\left[a_{i}, b_{i}\right]\right) \mathrm{d} a_{1} \\
& =\chi_{\lambda}\left(b_{1}^{-1} \cdot \prod_{i=2}^{g}\left[a_{i}, b_{i}\right]\right) \chi_{\lambda}\left(b_{1}\right) \frac{\operatorname{vol}(G)}{\chi_{\lambda}(1)} .
\end{aligned}
$$

Applying Proposition 6.8 yields

$$
\begin{aligned}
\int_{G^{2}} \chi_{\lambda}\left(\prod_{i=1}^{i=g}\left[a_{i}, b_{i}\right]\right) \mathrm{d} a_{1} \mathrm{~d} b_{1} & =\frac{\operatorname{vol}(G)}{\chi_{\lambda}(1)} \int_{G} \chi_{\lambda}\left(b_{1}\right) \chi_{\lambda}\left(b_{1}^{-1} \cdot \prod_{i=2}^{g}\left[a_{i}, b_{i}\right]\right) \mathrm{d} b_{1} \\
& =\left(\frac{\operatorname{vol}(G)}{\chi_{\lambda}(1)}\right)^{2} \chi_{\lambda}\left(\prod_{i=2}^{g}\left[a_{i}, b_{i}\right]\right) .
\end{aligned}
$$

Thus we see by induction that

$$
\int_{G^{2 g}} \chi_{\lambda}\left(\prod_{i=1}^{i=g}\left[a_{i}, b_{i}\right]\right) \mathrm{d} a_{1} \mathrm{~d} b_{1} \ldots \mathrm{d} a_{g} \mathrm{~d} b_{g}=\left(\frac{\operatorname{vol}(G)}{\chi_{\lambda}(1)}\right)^{2 g} \chi_{\lambda}(1) .
$$


Thus using $\chi_{\lambda}(1)=\operatorname{dim} V_{\lambda}$, we obtain

$$
\int_{\mu^{-1}(1)} \nu=\sum_{\lambda \in \widehat{G}} \frac{\chi_{\lambda}(1)^{2}}{\operatorname{vol}(G)}\left(\frac{\operatorname{vol}(G)}{\chi_{\lambda}(1)}\right)^{2 g}=\sum_{\lambda \in \widehat{G}} \frac{(\operatorname{vol}(G))^{2 g-1}}{\left(\operatorname{dim} V_{\lambda}\right)^{2 g-2}} .
$$

which is what we wanted to prove Q.E.D.

\subsection{Comments, references and further read- ings}

The construction of the symplectic form in the compact case is given in AtiyahBott [AB83]. It was preceded by unpublished construction by Narasimhan. Goldman extended in [Gol84] this construction to non compact groups and made the link with Weil-Petersson symplectic form.

The Goldman algebra and the brackets of Wilson loops is given by Goldman in [Gol86], and this formula was preceded and inspired by computations of Wolpert in Teichmüller theory [Wol83]. Later on, Turaev [Tur91] exhibited the bialgebra structure on the Goldman algebra and made the link with the skein relations appearing in Witten's construction of topological quantum field theories [Wit88].

Goldman algebra was extended in higher dimensions to string topology as developed by Chas and Sullivan[CS04, Cha10]. Related construction were also found by the author of these notes in [Lab10].

Spin networks were constructed by Penrose, but it is hard to pinpoint the correct reference. Mathematical references about spin networks are Baez [Bae96] and Lawton-Peterson [LP09]. The relation with the symplectic form is in Fock-Rosly [FR99]. Finally, other classes of observables encompassing both wilson loops and spin networks and which are "universal in the groups" are the chord diagram of Andersen-Mattes-Reshetikhin [AMR98, AMR96].

The computation of the volumes of the character variety in the compact case is due to Witten [Wit91]. These computations are related to - and help to prove - Verlinde formulae via a symplectic approach. The case of $\mathrm{SU}(2)$ is done by Donaldson in [Don93], $\mathrm{SU}(n)$ by Jeffrey-Kirwan [JK95] and the general case by Bismut-Labourie [BL99].

The Riemann moduli space which is a connected component of

$$
\operatorname{Rep}\left(\pi_{1}(S), \operatorname{PSL}(2, \mathbb{R}) / \operatorname{Out}\left(\pi_{1}(S)\right)\right.
$$

where $\operatorname{Out}\left(\pi_{1}(S)\right.$ is the mapping class group is also of finite volume. Mirzakhani computed recursive formulae for these volumes in [Mir07a] and later used 
the same symplectic approach as was used for Verlinde formulae to compute intersection numbers on the Riemann moduli space and give another proof of Witten's conjecture proved by Kontsevich in [Mir07b].

On the contrary the moduli space, $\operatorname{Rep}\left(\pi_{1}(S), \operatorname{PSL}(n, \mathbb{R}) / \operatorname{Out}\left(\pi_{1}(S)\right)\right.$ can be proved to have infinite volume when $n>2$ using generalised MirzakhaniMcShane's identities [LM09]. 


\section{Chapter 7}

\section{3-manifolds and integrality questions}

In this chapter, we shall prove the integrality of the Atiyah-Bott-Goldman form. Moreover we will explain that 3-manifolds bordered by a surface give rise to Lagrangian submanifolds of the character variety. We interpret the Chern-Simons invariant of a 3-manifold obtained by gluing along a surface as a symplectic invariant in the character variety.

Recall now that Lagrangian submanifolds in the cotangent space of a manifold $\mathrm{T}^{*} M$ are -very roughly speaking- the graph of the differential of a function on $M$. Thus a 3-manifold bordered by a surface should be considered as function on a "ghost configuration space" of which $\mathcal{M}(S, G)$ would be the cotangent space. Quantising this picture, Witten launched topological quantum field theory which is still a very active field of research - see the last section for references.

\subsection{Integrality}

We prove in this section

Proposition 7.1.1 [The SYMPLECTIC FORM IS INTEGRAL] Let $\omega$ be the symplectic form on $\mathcal{M}^{z d}(S, G)$. For any map from $f$ to the 2-sphere $S^{2}$ to $\mathcal{M}^{z d}(S, G)$, we have

$$
\frac{1}{4 \pi^{2}} \int_{S^{2}} f^{*} \omega \in \mathbb{Z}
$$

More generally, the symplectic form on $\mathcal{M}^{z d}(S, G)$ satisfies

$$
\frac{1}{4 \pi^{2}} \omega \in H^{2}\left(\mathcal{M}^{z d}(S, G), \mathbb{Z}\right)
$$




\section{REMARKS:}

- We have not defined - and will not - what is $H^{2}\left(\mathcal{M}^{z d}(S, G), \mathbb{Z}\right)$. Accordingly, we shall only prove the first statement of the previous proposition.

- It follows from this proposition and a little more work that the symplectic structure suitably normalised can be realised as the first Chern class of a line bundle over $\mathcal{M}^{z d}(S, G)$ : more precisely, there exists a complex line bundle $L$ over $\mathcal{M}^{z d}(S, G)$ and a connection $\nabla$ on $L$ with curvature $R^{\nabla}$ such that

$$
\frac{i}{2 \pi} \operatorname{Tr}\left(R^{\nabla}\right)
$$

is cohomologous to $\frac{1}{4 \pi^{2}} \omega$. For a general symplectic manifold with integral symplectic form, this line bundle is in general not unique. We comment on that at the end of the chapter.

\subsubsection{The Cartan form}

We continue to assume that $G$ is a simply-connected simple compact Lie group and we fix an invariant non-degenerate bilinear form $\langle\cdot, \cdot\rangle$ on $\mathfrak{g}$.

Definition 7.1.2 We define the Cartan 3-form $\omega_{C}$ as the left-invariant 3-form on $G$ given by

$$
\omega_{C}(A, B, C):=\langle[A, B], C\rangle
$$

for $A, B, C$ in $\mathfrak{g}$.

The form $\frac{1}{4 \pi^{2}} \omega_{C}$ satisfies the following property: for any smooth map $F$ : $M \rightarrow G$ from a 3 -dimensional manifold $M$ into $G$ one has

$$
\frac{1}{4 \pi^{2}} \int_{M} F^{*} \omega_{C} \in \mathbb{Z}
$$

The reader is invited to check this property at least when $G=\mathrm{SU}(2)$ or $G=$ $\mathrm{SO}(3)$. The general then follows from the fact that the 3-integral homology of a group is generated by the $G=\mathrm{SU}(2)$ or $G=\mathrm{SO}(3)$ sitting as subgroups in $G$, fact for which I could not find an easy reference, nevertheless a proof of the integrality of this form is a consequence of Borel general results, [Bor53] as well as the construction of Chern-Simons invariants [CS74]. 


\subsubsection{Integrating the Cartan form}

As a convention in this section, if $q$ is a bilinear form on a vector space $V$ with values in $W$, and if $\alpha$ and $\beta$ are 1 -forms with values in $V, q(\alpha \wedge \beta)$ is the 2 -form with values in $W$ given by

$$
q(\alpha \wedge \beta)=q(\alpha(u), \beta(v))-q(\alpha(v), \beta(u)) .
$$

Lemma 7.1.3 Let $M=S \times \mathbb{T}^{1}$, where $S$ is a surface. Let $F$ be a map from $M$ to $G$. For $t$ in $[0,1]$, we write $F_{t}(m)=F(m, t)$ and denote by $F_{t}^{-} 1: M \rightarrow G$ the map

$$
m \mapsto\left(F_{t}(m)\right)^{-1} \text {. }
$$

We have

$$
\int_{S \times \mathbb{T}^{1}} F^{*} \omega_{C}=\int_{\mathbb{T}^{1}}\left(\int_{S}\left\langle\left. F_{s}^{-1} d F_{s} \wedge \frac{\mathrm{d}}{\mathrm{d} t}\right|_{t=s}\left(F_{t}^{-1} d F_{t}\right)\right\rangle\right) \mathrm{d} s
$$

Proof: We first give a detailed pedestrian proof, where we consider $G$ as a group - via the adjoint representation - of $n \times n$-matrices. Therefore, if $s$ belongs to $[0,1]$, we denote by $\dot{F}_{s}$ the map from $S$ to $M_{n}(\mathbb{R})$ given by

$$
\dot{F}_{s}:\left.m \rightarrow \frac{\mathrm{d}}{\mathrm{d} t}\right|_{t=s} F_{t}
$$

We start from the integrand in the right hand side of the equation. Applying the chain rule yields

$$
\begin{aligned}
& \int_{S} \operatorname{Tr}\left(\left.F_{s}^{-1} d F_{s} \wedge \frac{\mathrm{d}}{\mathrm{d} t}\right|_{t=s}\left(F_{t}^{-1} d F_{t}\right)\right) \\
= & \int_{S} \operatorname{Tr}\left(F_{s}^{-1} d F_{s} \wedge F_{s}^{-1} \mathrm{~d} \dot{F}_{s}\right) \\
- & \int_{S} \operatorname{Tr}\left(F_{s}^{-1} d F_{s} \wedge\left(F_{s}^{-1} d F_{s}\left(\partial_{t}\right) F_{s}^{-1} d F_{s}\right)\right)
\end{aligned}
$$

Now, we observe that for $u$ and $v$ in $\mathrm{T}_{m} S$, we have

$$
\begin{aligned}
\left(F_{s}^{-1} d F_{s} \wedge F_{s}^{-1} \mathrm{~d} \dot{F}_{s}\right)(u, v) & =F_{s}^{-1} d F_{s}(u) F_{s}^{-1} \mathrm{~d} \dot{F}_{s}(v)-F_{s}^{-1} d F_{s}(v) F_{s}^{-1} \mathrm{~d} \dot{F}_{s}(u) \\
& =-\mathrm{d}\left(\operatorname{Tr}\left(F^{-1} \mathrm{~d} \dot{F}\right)\right)(u, v) .
\end{aligned}
$$

Hence, by Stokes Formula

$$
\int_{S} \operatorname{Tr}\left(F_{s}^{-1} d F_{s} \wedge F_{s}^{-1} \mathrm{~d} \dot{F}_{s}\right)=-\int_{S} \mathrm{~d}\left(\operatorname{Tr}\left(F^{-1} \mathrm{~d} \dot{F}\right)\right)=0 .
$$


Moreover, observe that the pullback of $\omega_{C}$ by $F$ is

$$
\begin{aligned}
F^{*} \omega_{C}(u, v, w) & =\operatorname{Tr}\left(F^{-1} d F(u) F^{-1} d F(v) F^{-1} d F(w)\right) \\
& -\operatorname{Tr}\left(F^{-1} d F(v) F^{-1} d F(u) F^{-1} d F(w)\right) .
\end{aligned}
$$

It follows that if $u$ and $v$ belongs to $\mathrm{T}_{m} S$, we have

$$
\operatorname{Tr}\left(F_{s}^{-1} d F_{s} \wedge\left(F_{s}^{-1} d F_{s}\left(\partial_{t}\right) F_{s}^{-1} d F_{s}\right)\right)(u, v)=F^{*} \omega_{C}\left(u, v, \partial_{t}\right)
$$

Finally, combining Equations (7.1), (7.2) and (7.3) we obtain the assertion.

We now give a short proof which is just a conceptual way to write down the above pedestrian computation. Let $\mu$ be the Maurer-Cartan 1-form on $G$ with value in $\mathfrak{g}$ given by $\mu_{g}(u)=g^{-1} u$. The Maurer-Cartan form satisfies

$$
\mathrm{d} \mu+\frac{1}{2}[\mu \wedge \mu]=0,
$$

We now use freely the Lie-Cartan calculus. Then the integrand in the righthand term can be written as

$$
\int_{S}\left\langle\left. F_{s}^{-1} d F_{s} \wedge \frac{\mathrm{d}}{\mathrm{d} t}\right|_{t=s}\left(F_{t}^{-1} d F_{t}\right)\right\rangle=\int_{S}\left\langle F^{*} \mu \wedge L_{\partial_{t}} F^{*} \mu\right\rangle
$$

Using the fundamental formula of the Lie-Cartan formalism

$$
L_{X}=i_{X} d+d i_{X}
$$

Stokes Formula and Equation 7.3, we obtain

$$
\begin{aligned}
\int_{S}\left\langle F^{*} \mu \wedge L_{\partial_{t}} F^{*} \mu\right\rangle & =\frac{1}{2} \int_{S} L_{\partial_{t}} F^{*}\langle\mu \wedge \mu\rangle \\
& \left.=\frac{1}{2} \int_{S} i_{\partial_{t}} F^{*}\langle\mu \wedge[\mu \wedge \mu])\right\rangle .
\end{aligned}
$$

Since

$$
\langle\mu \wedge[\mu \wedge \mu])\rangle=\omega_{C},
$$

this concludes the proof of our proposition Q.E.D.

\section{Proof of Proposition 7.1.1}

We first prove that for any map $f$ from the sphere $S^{2}$ to $\mathcal{M}^{z d}(S, G)$ whose range contains the trivial connection, we have that

$$
\int_{S^{2}} f^{*} \omega \in 4 \pi^{2} \mathbb{Z} .
$$


Then we shall explain the idea of the general proof.

Let $m_{0}$ be a point in $S^{2}$ which is mapped to the trivial connection. We describe now the sphere $S^{2}$ as $S^{2}=D / \partial D$, where the boundary of the disc is mapped to $m_{0}$. As $D$ is contractible, the map $f: S^{2} \rightarrow \mathcal{M}^{z d}(S, G)$ can be lifted to a map $F: D \rightarrow \mathcal{A}_{E}$, where the latter denotes the space of flat connection on $E$ - as in the proof of Theorem 6.2.2. In this proof, we have seen that the symplectic form on $\mathcal{M}^{z d}(S, G)$ is obtained locally as the pullback of a certain form $\Omega$ via a smooth local section. This implies that

$$
\int_{S^{2}} f^{*} \omega=\int_{D} F^{*} \Omega
$$

Recall from the proof of Theorem 6.2.2 that $\Omega=\mathrm{d} \beta$, where

$$
\beta_{\nabla}(A)=\int_{S} \operatorname{tr}\left(\left(\nabla-\nabla_{0}\right) \wedge A\right)
$$

for any fixed choice of connection $\nabla_{0}$ on $E$. Thus Stokes' theorem yields

$$
\int_{S^{2}} f^{*} \omega=\int_{\partial D} F^{*} \beta
$$

Since $\partial D$ is crushed to $m_{0}, F$ maps $\partial D$ to the fibre over $f\left(m_{0}\right)$ of the infinitedimensional bundle $\mathcal{A}_{E} \rightarrow \mathcal{M}^{z d}(S, G)$. This fibre is identified with the gauge group of $E$ and as $E$ was assumed to be trivial this gauge group is just the group of smooth maps from $S$ to $G$. In particular, we have a smooth map

$$
H: S \times \partial \mathrm{D} \rightarrow G, \quad(x, \theta) \mapsto H_{\theta}(x)
$$

such that

$$
F(t)=\mathrm{D}^{\theta}:=H_{\theta}^{-1} \mathrm{D} H_{\theta},
$$

where $\mathrm{D}$ denotes the trivial connection on $E$. More precisely our notation means that for any vector $X$ tangent to $S$ and section $u$ of $E$, we have

$$
\mathrm{D}_{X}^{\theta}(u)=H_{\theta}^{-1}\left(\mathrm{D}_{X}\left(H_{\theta}(u)\right)\right) .
$$

In particular, we have by the definition of $\beta$ that

$$
\int_{S^{2}} f^{*} \omega=\int_{\partial D} F^{*} \beta=\int_{\partial D}\left(\int_{S \times \partial D} \operatorname{Tr}\left(\left(\mathrm{D}-\mathrm{D}^{\theta}\right) \wedge \frac{d D^{\theta}}{\mathrm{d} \theta}\right) \mathrm{d} \theta\right.
$$

Now $\mathrm{D}-\mathrm{D}^{\theta}=H_{t}^{-1} d H_{t}$ and $\dot{\mathrm{D}}_{\theta}=\frac{\partial}{\partial \theta}\left(H_{\theta}^{-1} d H_{\theta}\right)$ and thus by Lemma 7.1.3,

$$
\int_{S^{2}} f^{*} \omega=\int_{\partial D}\left(\int_{S} \operatorname{Tr}\left(H_{\theta}^{-1} d H_{\theta} \wedge \frac{\partial}{\partial \theta}\left(H_{\theta}^{-1} d H_{\theta}\right)\right)\right) \mathrm{d} \theta
$$




$$
=\int_{S \times \partial D} H^{*} \omega_{C} \in 4 \pi^{2} \mathbb{Z} .
$$

This finishes the proof of the first part of the proposition.

We explain briefly how to obtain the second part using a similar scheme.

Let $\Sigma$ be a triangulated closed surface. Using the previous argument, we can lift every simplex $\Delta$ to a simplex $\widetilde{\Delta}$ in $\mathcal{A}_{E}$. Then, by construction, the integral of $\omega$ on $\Delta$ is the integral of the symplectic form $\Omega$ of $\mathcal{A}_{E}$ on $\tilde{\Delta}$. Let $e$ be an edge of the triangulation, the lift of the simplex on each side of the triangulation do not coincide on the boundary and the defect is given by a map from $e$ to the gauge group. Since the gauge group is connected, we can find a homotopy of this map to the identity. In other words, we can find a map from $e \times[0,1]$ to $\mathcal{A}_{E}$ which connects the two lifts of the two simplexes bounded by $e$ such that the image of $\{a\} \times[0,1]$ lies in the orbit of the gauge group for each $a$ in $e$. Observe now that the integral of $\Omega$ on the image of this map is zero. It follows that - after "thickening" the edge, we have constructed a map $\Phi$ from $\Sigma \backslash V$ - where $V$ is the set of vertices of the triangulation - in $\mathcal{E}$ so that

$$
\int_{\Sigma \backslash V} \Phi^{*} \Omega=\int_{\Sigma} f^{*} \omega
$$

We now use as before the fact that $\Omega=\mathrm{d} \beta$ and obtain that

$$
\int_{\Sigma \backslash V} \Phi^{*} \Omega=\sum_{v \in V} \int_{c_{v}} \beta,
$$

where, for each vertex $v, c_{v}$ is a curve in an orbit of the gauge group. Just as before, we obtain a map $H$ from $S \times[0,1]$ to $G$ so that

$$
\int_{c_{v}} \beta=\int_{M \times[0,1]} H^{*} \omega_{C} \in 4 \pi^{2} \mathbb{Z}
$$

This shows that

$$
\int_{\Sigma} f^{*} \omega \in 4 \pi^{2} \mathbb{Z}
$$

and concludes this sketch of proof. Q.E.D.

\subsection{Boundary of 3-Manifolds and Lagrangian submanifolds}

Let $M$ be a 3-manifold such that $\partial M=S$. The inclusion from $S$ into $M$ gives rise to a map $i_{*}$ from $\pi_{1}(S)$ to $\pi_{1}(M)$, hence to a map

$$
I: \operatorname{Rep}\left(\pi_{1}(M), G\right) \rightarrow \operatorname{Rep}\left(\pi_{1}(S), G\right) .
$$


Let $\mathcal{L}_{M}=I\left(\operatorname{Rep}\left(\pi_{1}(M), G\right)\right)$ and $\mathcal{L}_{M}^{z d}:=\mathcal{L}_{M} \cap \mathcal{M}^{z d}(S, G)$. In other words

$$
\mathcal{L}_{M}^{z d}:=\left\{[\rho] \in \mathcal{M}^{z d}(S, G)\left|\exists \widetilde{\rho}: \pi_{1}(M) \rightarrow G, \rho=\widetilde{\rho}\right|_{\pi_{1}(S)}\right\}
$$

Theorem 7.2.1 [LAGRANGIAn SUBMANifold] Let $M$ be an 3-manifold with boundary $S$, then $\mathcal{L}_{M}^{z d}$ is a Lagrangian submanifold of $\mathcal{M}^{z d}(S, G)$.

\subsubsection{Submanifolds of the moduli space}

We first prove

Proposition 7.2.2 Let $M$ be an 3-manifold with boundary $S$, then $\mathcal{L}_{M}^{z d}$ is a smooth submanifold of $\mathcal{M}^{z d}(S, G)$ with

$$
\operatorname{dim} \mathcal{L}_{M}=\frac{1}{2} \operatorname{dim} \mathcal{M}^{z d}(S, G) .
$$

Proof: We shall use freely result about relative cohomology that can be found in Hatcher for instance.

We only consider smooth points. Let $\rho$ be a smooth point of $\operatorname{Rep}\left(\pi_{1}(M), G\right)$ and $I: \operatorname{Rep}\left(\pi_{1}(M), G\right) \rightarrow \mathcal{M}(S, G)$ as above. Then, by definition

$$
\operatorname{ker}(\mathrm{T} I)=H_{\rho}^{1}(M, \partial M)
$$

where by definition $H_{\rho}^{1}(M, \partial M)$ is represented by forms vanishing on a neighbourhood of $\partial M$. Then, by Poincaré duality [Hat02], the map

$$
H_{\rho}^{i}(M, \partial M) \times H_{\rho}^{3-i}(M) \rightarrow \mathbb{R},(\alpha, \beta) \mapsto \int_{M} \operatorname{Tr}(\alpha \wedge \beta),
$$

is a duality. Thus

$$
\operatorname{dim}(\operatorname{ker}(\mathrm{T} I))=\operatorname{dim}\left(H_{\rho}^{1}(M, \partial M)\right)=\operatorname{dim}\left(H_{\rho}^{2}(M)\right) .
$$

Thus

$$
\operatorname{dim}\left(\Im(\mathrm{T} I)=\operatorname{dim}\left(H_{\rho}^{1}(M)\right)-\operatorname{dim}\left(H_{\rho}^{2}(M)\right)=\chi(M) \operatorname{dim}(\mathfrak{g}) .\right.
$$

For this last equality we used the fact - true in full generality but proved for closed surfaces in 2.5.17 - that the Euler characteristic for the twisted cohomology is equal to the dimension of the generic fibre multiplied by the Euler characteristic of the manifold. 
Finally, it remains to prove that $2 \chi(M)=\chi(\partial M)$. But this comes from the long exact sequence for relative cohomology

$$
\begin{aligned}
& 0 \rightarrow H^{2}(M, \partial M) \rightarrow H^{2}(M) \rightarrow H^{2}(\partial M) \\
& \rightarrow H^{1}(M, \partial M) \rightarrow H^{1}(M) \rightarrow H^{1}(\partial M) \rightarrow 0,
\end{aligned}
$$

and Poincaré duality which shows that

$$
\begin{aligned}
& \operatorname{dim}\left(H^{2}(M, \partial M)\right)=\operatorname{dim}\left(H^{1}(M)\right) \\
& \operatorname{dim}\left(H^{2}(M, \partial M)\right)=\operatorname{dim}\left(H^{1}(M)\right) .
\end{aligned}
$$

Q.E.D.

\subsubsection{Isotropic submanifold}

We prove the second part of Theorem 7.2.1.

Proposition 7.2.3 The restriction of the symplectic form $\left.\omega\right|_{\mathcal{L}_{M}^{z d}}$ vanishes identically.

REMARK: Actually, this statement generalises when $M$ is not a handlebody. The same result - and proof - holds when we replace $\left.\omega\right|_{\mathcal{L}_{M}^{z d}}-$ which is not necessarily a submanifold - by any smooth submanifold embedded in $\left.\omega\right|_{\mathcal{L}_{M}^{z d}}$.

Proof: Suppose that $\left.A, B \in \mathrm{T}_{[} \rho\right] \mathcal{M}^{z d}(S, G) \cong H_{\rho}^{1}(S)$ are contained in $\left.\mathrm{T}_{[} \rho\right] \mathcal{L}_{M}^{z d}$. Then they can be extended to cohomology classes $\widetilde{A}, \widetilde{B}$ in $H_{\rho}^{1}(M)$ and

$$
\omega_{\rho}(A, B)=\int_{S} \operatorname{Tr}(A \wedge B)=\int_{M} \mathrm{~d} \operatorname{Tr}(\widetilde{A} \wedge \widetilde{B})=0 .
$$

Q.E.D.

This means that $\mathcal{L}_{M}^{z d}$ is a Lagrangian submanifold of $\mathcal{M}^{z d}(S, G)$ in the sense of the following definition:

Definition 7.2.4 If $(M, \omega)$ is a symplectic manifold and $N \subset M$ is a submanifold, then $N$ is called Lagrangian if it is maximally degenerate, in other words $\operatorname{dim} N=\frac{1}{2} \operatorname{dim} M$ and $\left.\omega\right|_{N} \equiv 0$.

\subsubsection{Chern-Simons invariants}

In this final paragraph, we discuss some application of the moduli spaces $\mathcal{M}(S, G)$ for $G=\mathrm{SU}(n)$ to 3 -manifolds. 


\section{Chern-Simons invariants for connections and representations}

Let $E$ be a rank $n$ complex vector bundle over a 3-manifold $M$. By choosing a hermitian structure on $E$ we can view $E$ as a $G$-bundle for $G=\operatorname{SU}(n)$. Denote by $\mathcal{A}_{E}$ the affine space of flat $G$-connections on $E$ and by $A_{E}$ the underlying vector space. We view $\mathcal{A}_{E}$ as an infinite-dimensional manifold with tangent space $A_{E}$ at each point.

Definition 7.2.5 [CHERn-Simons FORM] We define the Chern-Simons form as the real valued 1 -form $\omega^{C S}$ on the affine space $\mathcal{A}_{E}$ given by

$$
\omega_{\nabla}^{C S}(A):=\int_{M} \operatorname{Tr}\left(A \wedge R^{\nabla}\right)
$$

where $\nabla$ is a G-connection, $A$ is an element of $A_{E} \cong \mathrm{T}_{\nabla}\left(\mathcal{A}_{E}\right)$, where $R^{\nabla}$ is the curvature of $\nabla$ and we set

$$
\left(A \wedge R^{\nabla}\right)\left(X_{1}, X_{2}, X_{3}\right):=\frac{1}{6} \sum_{\sigma \in \mathfrak{S}(3)} A\left(X_{\sigma(1)}\right) R^{\nabla}\left(X_{\sigma(2)}, X_{\sigma(3)}\right) .
$$

Proposition 7.2.6 Assume that the 3-manifold $M$ is closed. Suppose $\nabla_{1}$ and $\nabla_{2}$ are gauge-equivalent connections on $E$ and $\gamma:[0,1] \rightarrow \mathcal{A}_{E}$ is a path from $\nabla_{1}$ to $\nabla_{2}$. Then

$$
\frac{1}{4 \pi^{2}} \int_{\gamma} \omega^{C S} \in \mathbb{Z}
$$

In particular, $\omega^{C S}$ is closed.

We shall prove this proposition shortly. As a first consequence of this proposition, when the 3-manifold is closed, we can define the Chern-Simons action difference for connections.

Definition 7.2.7 [CHERN-Simons ACTION FOR CONNECTIONS] Let $\nabla_{0}$ and $\nabla_{1}$ be two connections defined over the same vector bundle $E$. Then we define the Chern-Simons action difference to be the real number

$$
C S\left(\nabla_{1}, \nabla_{0}\right)=\frac{1}{4 \pi^{2}} \int_{\gamma} \omega^{C S}\left(\dot{\nabla}_{t}\right) d t
$$

where $\gamma$ is any path of connections joining $\nabla_{0}$ to $\nabla_{1}$.

As a second consequence, we can define the Chern-Simons action difference for gauge equivalence classes of connections - and in particular for representations from $\pi(M)$ to $\mathrm{SU}(n)$ - as an element of $\mathbb{R} / \mathbb{Z}$, in the following way 
Definition 7.2.8 [CHERN-SimONS ACTION FOR EQUIVALENCE CLASSES OF CONNECTIONS] Let $\rho_{1}$ and $\rho_{2}$ be two gauge equivalence classes of connections defined over the same vector bundle $E$. Let $\nabla_{1}$ and $\nabla_{2}$ be two connections on $E$ representing respectively $\rho_{1}$ and $\rho_{2}$, then we define the Chern-Simons action difference to be

$$
C S\left(\rho_{1}, \rho_{2}\right)=C S\left(\nabla_{1}, \nabla_{2}\right) \in \mathbb{R} / \mathbb{Z}
$$

Observe that, whenever it makes sense,

$$
C S\left(\rho_{1}, \rho_{2}\right)=C S\left(\rho_{1}, \rho_{3}\right)+C S\left(\rho_{3}, \rho_{2}\right) .
$$

We now prove Proposition 7.2.6.

Proof: Define a bundle $\mathcal{E}$ over $M \times[0,1]$ by $\mathcal{E}:=p_{1}^{*}(E)$, where $p_{1}$ denotes the projection onto the first factor. Then there exists a unique connection $\nabla$ on $\mathcal{E}$ which satisfies the following two conditions:

1. Let $i_{t}: M \rightarrow M \times\{t\}$ be the injection $m \rightarrow(m, t)$ then $i^{*} \nabla=\gamma(t)$.

2. Let $j_{m}:[0,1] \rightarrow\{m\} \times[0,1]$ be the injection $t \rightarrow(m, t)$. Observe that by construction $j_{m}^{*} \mathcal{E}$ is the trivial bundle $\left.\mathcal{E}\right|_{m \times[0,1]}$. We furthermore require that for each fixed $m$ in $M, J_{m}^{*} \nabla$ is the trivial connection.

Moreover, the curvature of $\nabla$ is given by

$$
R^{\nabla}=d t \wedge \dot{\nabla}+R^{\gamma(t)}
$$

Therefore,

$$
\int_{\gamma} \omega^{C S}=\int_{0}^{1}\left(\int_{M} \operatorname{Tr}\left(\dot{\nabla} \wedge R^{\gamma(t)}\right)\right) \mathrm{d} t=\frac{1}{2} \int_{M \times[0,1]} \operatorname{Tr}\left(R^{\nabla} \times R^{\nabla}\right) .
$$

Now consider $P:=M \times \mathbb{T}^{1}$, which we view as a quotient of $M \times[0,1]$ by identifying the endpoints of the interval. Using the gauge equivalence between $\nabla_{1}$ and $\nabla_{2}$, we can identify $\left.\mathcal{E}\right|_{M \times\{0\}}$ and $\left.\mathcal{E}\right|_{M \times\{1\}}$ in order to obtain a bundle with connection $\left(\mathcal{E}^{\prime}, \nabla^{\prime}\right)$ over $P$. Then we have

$$
\frac{1}{4 \pi^{2}} \int_{\gamma} \omega^{C S}=\frac{1}{8 \pi^{2}} \int_{P} \operatorname{Tr}\left(R^{\nabla^{\prime}} \wedge R^{\nabla^{\prime}}\right)=p_{1}\left(\mathcal{E}^{\prime}\right),
$$

where $p_{1}$ denotes the first Pontryagin class. Then the proposition follows from the fact that the Pontryagin classes of the bundle $\mathcal{E}^{\prime}$ are integral. Q.E.D. 


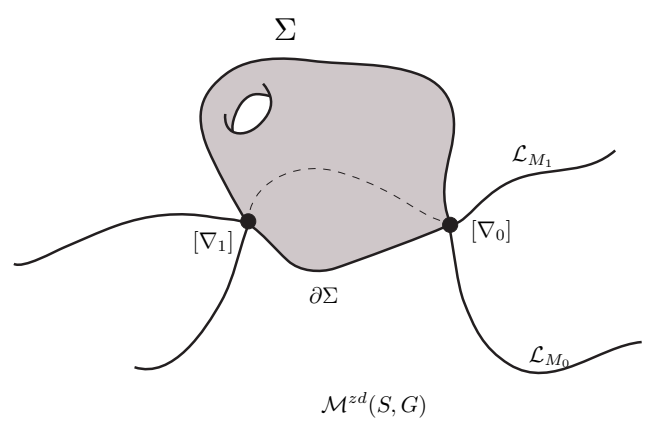

Figure 7.1: Connections in good position

\subsubsection{Chern Simons action difference and the symplec- tic form}

We explain how to compute the Chern-Simons action difference, using a decomposition of the 3-manifold and the symplectic geometry of the moduli space.

Let us first say that a splitting of the 3-manifold $M$ is a given by a surface $S$ of genus greater than 2 embedded in $M$, so that $M \backslash S=M_{1} \sqcup M_{2}$.

The following result result tells us that splitting exists

Proposition 7.2.9 [HeEgaARD SPLitTing] If $M$ is an arbitrary 3-manifold, then there exists handlebodies $M_{1}$ and $M_{2}$ and a homeomorphism $f: \partial M_{1} \rightarrow$ $\partial M_{2}$ such that

$$
M=M_{1} \cup_{f} M_{2}
$$

The surface appearing as the common boundary of the two handlebodies is constructed as the boundary of tubular neighbourhood of the 1-skeleton of a triangulation of the 3-manifold. The observation is then that this surface is also the boundary of the tubular neighbourhood of the 1-skeleton of the dual graph. Such a decomposition can also be obtained using a Morse function

Clearly a flat connection on $M$ restricts to flat connections on $M_{1}$ on $M_{2}$ and vice-versa. Thus the gauge equivalence classes of flat connection on the 3-manifold $M$ are parametrised by $\mathcal{L}_{M_{1}} \cap \mathcal{L}_{M_{2}} \subset \mathcal{M}(S, G)$. We will apply this fact in the next subsection in order to compute an important invariant for flat connections on 3-manifolds.

For this we fix a splitting $M=M_{1} \cap_{f} M_{0}$, where $M_{1}, M_{0}$ are handlebodies with boundary $S$. Then $\nabla_{1}, \nabla_{0}$ correspond to points in $\mathcal{L}_{M_{1}} \cap \mathcal{L}_{M_{0}} \subset \mathcal{M}(S, G)$. Let us make a number of simplifying assumptions: 
- The connections $\nabla_{1}, \nabla_{0}$ correspond to regular points in $\mathcal{M}^{z d}(S, G)$.

- There is a surface $\Sigma$ with one connected boundary component with two marked points $x_{1}, x_{0}$ on the boundary and a smooth map $\iota: \Sigma \rightarrow$ $\mathcal{M}^{z d}(S, G)$ such that $\iota\left(x_{j}\right)$ corresponds to $\nabla_{j}$ and that the two components of $\partial \Sigma \backslash\left\{x_{1}, x_{0}\right\}$ are mapped to $\mathcal{L}_{M_{1}}$ and $\mathcal{L}_{M_{0}}$ respectively.

Then one has a symplectic way to compute the Chern-Simons invariant

Theorem 7.2.10 Let $\nabla_{1}, \nabla_{0}$ be flat connections on $M$ satisfying the above regularity requirements and defining a surface $\Sigma$ in $\mathcal{M}^{z d}(S, G)$ as above. Then

$$
\operatorname{CS}\left(\left[\nabla_{1}\right],\left[\nabla_{0}\right]\right)=\frac{1}{4 \pi^{2}} \int_{\Sigma} \omega
$$

as elements of $\mathbb{R} / \mathbb{Z}$.

As an exercise, we leave the reader go through the proof below and check which orientation on $S$ - either as a boundary of $M_{0}$ or as a boundary of $M_{1}$ and which orientation on $\Sigma$ gives the correct sign in the formula above.

Proof: We only sketch this proof. We triangulate $\Sigma$ in such a way that $x_{1}$ and $x_{0}$ are the only vertices of this triangulation that belong to the boundary. Let $b_{1}$ and $b_{0}$ be the two edges of this triangulation in the boundary of $\Sigma$, where $b_{1}$ joins $x_{0}$ to $x_{1}$.

Proceeding as alluded in the end of the proof of Proposition 7.1.1, we construct a map $\Phi$ from $\Sigma \backslash V$ - where $V$ is the set of vertices of the triangulation - in $\mathcal{A}_{E}$ lifting $\iota$ so that

$$
\int_{\Sigma \backslash V} \Phi^{*} \Omega=\int_{\Sigma} \iota^{*} \omega
$$

Moreover, blowing up $x_{1}$ and $x_{0}$ to $\operatorname{arcs} c_{1}$ and $c_{0}$ and the interiors points of $V$ to loops $c_{v}$ all lying in orbits on the gauge group, we obtain that

$$
\int_{\Sigma \backslash V} \Phi^{*} \Omega=\sum_{v \in V \backslash\left\{x_{1}, x_{0}\right\}} \int_{c_{v}} \beta+\int_{c_{1}} \beta+\int_{c_{0}} \beta+\int_{b_{1}} \beta+\int_{b_{0}} \beta .
$$

Working in $\mathbb{R} / \mathbb{Z}$, and observing again that $\int_{c_{v}} \beta$ belongs to $\mathbb{Z}$, we are left to understanding the integral of $\beta$ along some arcs.

Since $\mathcal{A}_{E}$ is contractible, we can find a map $C$ from $[0,1] \times[0,1]$ filling up the loop given by $c_{1}, b_{1}, c_{0}$ and $b_{0}$. We choose this map so that $t \rightarrow C(0, t)$ is a parametrisation of $c_{1}$ with the respective obvious conventions for the other sides. We also denote by $\nabla_{s, t}$ the connection on the bundle $E$ over $S$ defined by $C(s, t)$. 
Therefore, it remains to compute

$$
\int_{C} \Omega=\int_{0}^{1}\left(\int_{0}^{1} \Omega\left(\partial_{s} C, \partial_{t} C\right) \mathrm{d} s\right) \mathrm{d} t
$$

By definition,

$$
\int \Omega\left(\partial_{s} C, \partial_{t} C\right) \mathrm{d} s=\int_{[0,1]} \int_{S} \operatorname{Tr}\left(\partial_{s} \nabla_{s, t} \wedge \partial_{t} \nabla_{s, t}\right) \mathrm{d} s .
$$

As usual, the map $t \rightarrow \nabla_{s, t}$ defines a connection $\nabla_{s}$ on $S \times[0,1]$ which restricts to $\nabla_{s, t}$ on $S \times\{t\}$ and to a trivial connection along $\{m\} \times[0,1]$. Thus

$$
R^{\nabla_{s}}=d t \wedge \partial_{t} \nabla_{s, t}+R^{\nabla_{s, t}}
$$

It follows that

$$
\int_{[0,1]} \int_{S} \operatorname{Tr}\left(\partial_{s} \nabla_{s, t}, \partial_{s} \nabla_{s t}\right)=\int_{S \times[0,1]} \operatorname{Tr}\left(\partial_{s} \nabla_{s, t}, R^{\nabla_{t}}\right) .
$$

Since $\nabla_{s, 0}$ and $\nabla_{s, 1}$ extends respectively to flat connections on $M_{0}$ and $M_{1}$. The connection $\nabla_{s}$ extends to a flat connection $\hat{\nabla}_{s}$ on $\hat{M}=M_{0} \cup S \times[0,1] \cup M_{1}$. We leave as an exercise that we can choose $\hat{\nabla}_{s}$ to be smooth (Hint : use local systems). Thus

$$
\begin{aligned}
\int_{[0,1]} \int_{S} \operatorname{Tr}\left(\partial_{s} \nabla_{s, t} \wedge \partial_{s} \nabla_{s, t}\right) & =\int_{S \times[0,1]} \operatorname{Tr}\left(\partial_{s} \nabla_{s} \wedge R^{\nabla_{s}}\right) \\
& =\int_{M} \operatorname{Tr}\left(\partial_{s} \hat{\nabla}_{s} \wedge R^{\hat{\nabla}_{s}}\right) \\
& =\omega_{\hat{M}}^{C S}\left(\partial_{s} \hat{\nabla}_{s}\right) .
\end{aligned}
$$

It follows that

$$
\frac{1}{4 \pi^{2}} \int_{C} \Omega=C S\left(\hat{\nabla}_{1}, \hat{\nabla}_{0}\right)
$$

By construction, the connection $\hat{\nabla}_{1}$ is gauge equivalent to $\nabla_{1}$, and $\hat{\nabla}_{0}$ is gauge equivalent to $\nabla_{0}$. Therefore the following identity holds in $\mathbb{R} / \mathbb{Z}$

$$
\frac{1}{4 \pi^{2}} \int_{C} \Omega=\omega_{\hat{M}}^{C S}\left(\nabla_{1}, \nabla_{0}\right)
$$

Q.E.D. 


\subsection{Comments, references and further read- ings}

The integrality of the Atiyah-Bott-Goldman symplectic form is usually proved by deep analytical methods in the context of Riemann surfaces: Quillen [Qui85] constructed a holomorphic determinant line bundle where the complex structure on $\mathcal{M}^{z d}(S, G)$ is induced from any choice of complex structure on $S$. Quillen also constructed a connection on that line bundle and proved that the curvature of this connection is a multiple of the Atiyah-Bott-Goldman symplectic form.

The construction of Chern-Simons invariant and more general secondary classes is due to Chern-Simons in [CS74]. The relation between Chern-Simons action and the intersection points of the Lagrangian submanifold in the character variety as is discussed in Atiyah's book [Ati90] which contains an enlightening introduction to Jones-Witten theory as well as in Kevin Walker's thesis [Wa192]. After Witten's seminal article [Wit89], Freed wrote several introduction to the role of Chern-Simons invariant in topological quantum field theories as well as classical field theories [Fre95, Fre09]. Relation between Heegaard splittings are discussed in [Hem76]. 


\section{Index}

$G$-structure, 50, 60

algebraic group, 98, 99

algebraic variety, 95

Betti number, 35, 73

boundary, 9, 146

Cartan, 141

Cayley graph, 26, 28

character, 136

character variety, 95, 101

Chern-Simons invariant, 148, 151, 153

chord diagrams, 138

cohomology (combinatorial), 37, 77, 98

cohomology (de Rham), 32, 35, 72, 73, 75,98

complex, 35, 78, 84

complex (acyclic), 84, 90

complex (combinatorial), 77

complex (de Rham), 35

complex (metric), 87, 89

complex (symplectic), 81, 89, 93

connected components, 109, 113, 115, 116

connection, 53, 54, 119

connection ( $G$-structure), 60

connection (discrete), 62, 65

connection (trivial), 54, 59, 61

contractible, 24, 36, 49

cover, 30, 46, 63-65

curvature, 59, 63, 70

determinant, 85, 93, 153

differential form, 32, 33, 37 differential form (closed), 35, 36, 73

differential form (exact), 35, 73

differential form (vector bundle valued), 53

duality and intersection, 41, 43, 72, 75, $82,121,146$

Euler characteristic, 39, 73, 146

fibre, 46

flat bundle and connection, 59, 63, 65, 150

fundamental group, 23, 25, 30, 123

fundamental group (cover version), 30

fundamental group (ribbon version), 29,63

gauge equivalence, $56,61,71$

gauge equivalence (discrete connections), 62

gauge equivalence (local systems), 64

gauge group, 46, 50, 56, 62, 64, 128

gluing, 9, 10, 14, 19, 40, 140

Goldman algebra, 124, 138

graph, 12

graph (geometric realisation), 12

graph (oriented), 12

group valued cocycle, 46, 50

Hamiltonian vector field, 122, 125

Heegard splitting, 150

holonomy (discrete connection), 63

holonomy (local systems), 64

holonomy of a path, 53, 57, 58, 63, 64

holonomy representation, 60, 61, 63 
homotopy, 24, 36, 49, 63

Hurewicz Theorem, 36, 40, 65, 66, 75

Lagrangian, 140, 147

Leibniz Identity, 54, 123

Lie algebra, 60, 122, 124, 131

Lie algebra bundle, 71, 75, 125

Lie group, 52, 98

line bundle, 46, 153

Liouville, 132

loacl system, 94, 119, 152

local system, 64, 65, 75

loop, 24, 29, 30, 123

mapping class group, 116, 139

Milnor-Wood inequality, 113

observable, 122, 129

parrallel section, 57,58

path, 25, 29, 30, 53, 57, 71, 148

path (discrete), 29, 30, 63

Poisson algebra, 122

Poisson bracket, 122

Poisson structure, 131

presentation 2-complex, 27, 28

pullback bundle, 52, 66, 119

pullback connection, 56

pullback of a form, 34

representation variety, 72,94

ribbon graph, 13, 14, 17, 18, 29, 30, $37,43,62,65$

ribbon graph (filling), 15, 40

section, 46

section along the curve, 49

spin network, 128, 129, 138

Stokes Formula, 36

surface group, 28, 29, 40

symplectic form, 41, 81

symplectic form (Atiyah-Bott-Goldman),

$121,140,151$ symplectic structure, 121, 138

torsion, 87, 93

trivial bundle, 50

trivialisation, 46, 48-50, 60, 61

vector bundle, 45, 52

vector bundle (morphism), 46, 50

vector bundle (rank), 46

vector bundle (total space), 45

Verlinde formulae, 138

Wilson loop, 123, 125, 129

Zariski topology, 95, 96, 101 


\section{Bibliography}

[AB83] Michael F Atiyah and Raoul Bott. The Yang-Mills equations over Riemann surfaces. Philos. Trans. Roy. Soc. London Ser. A, 308(1505):523-615, 1983.

[AMR96] Jørgen Ellegaard Andersen, Josef Mattes, and Nikolai Reshetikhin. The Poisson structure on the moduli space of flat connections and chord diagrams. Topology, 35(4):1069-1083, 1996.

[AMR98] Jørgen Ellegaard Andersen, Josef Mattes, and Nikolai Reshetikhin. Quantization of the algebra of chord diagrams. Math. Proc. Cambridge Philos. Soc., 124(3):451-467, 1998.

[Ati90] Michael Atiyah. The geometry and physics of knots. Lezioni Lincee. [Lincei Lectures]. Cambridge University Press, Cambridge, 1990.

[Bae96] John C. Baez. Spin networks in gauge theory. Adv. Math., 117(2):253-272, 1996.

[BGPG06] Steven B. Bradlow, Oscar García-Prada, and Peter B. Gothen. Maximal surface group representations in isometry groups of classical Hermitian symmetric spaces. Geom. Dedicata, 122:185-213, 2006 .

[BIW03] Marc Burger, Alessandra Iozzi, and Anna Wienhard. Surface group representations with maximal Toledo invariant. C. R. Math. Acad. Sci. Paris, 336(5):387-390, 2003.

[BL99] Jean-Michel Bismut and François Labourie. Symplectic geometry and the Verlinde formulas. In Surveys in differential geometry: differential geometry inspired by string theory, volume 5 of Surv. Differ. Geom., pages 97-311. Int. Press, Boston, MA, 1999.

[Bor53] Armand Borel. Sur la cohomologie des espaces fibrés principaux et des espaces homogènes de groupes de Lie compacts. Ann. of Math. (2), 57:115-207, 1953. 
[BT82] Raoul Bott and Loring W. Tu. Differential forms in algebraic topology, volume 82 of Graduate Texts in Mathematics. Springer-Verlag, New York, 1982.

[BtD85] Theodor Bröcker and Tammo tom Dieck. Representations of compact Lie groups. Number 98 in Graduate Texts in Mathematics. Springer-Verlag, 1985.

[Cha10] Moira Chas. Minimal intersection of curves on surfaces. Geom. Dedicata, 144:25-60, 2010.

[Che77] Jeff Cheeger. Analytic torsion and Reidemeister torsion. Untitled, 74(7):2651-2654, 1977.

[CS74] Shiing Shen Chern and James Simons. Characteristic forms and geometric invariants. Ann. of Math. (2), 99:48-69, 1974.

[CS04] Moira Chas and Dennis Sullivan. Closed string operators in topology leading to Lie bialgebras and higher string algebra. In The legacy of Niels Henrik Abel, pages 771-784. Springer, Berlin, 2004.

[Das92] Georgios D. Daskalopoulos. The topology of the space of stable bundles on a compact Riemann surface. J. Differential Geom., 36(3):699-746, 1992.

[Don93] S. K. Donaldson. Gluing techniques in the cohomology of moduli spaces. In Topological methods in modern mathematics (Stony Brook, NY, 1991), pages 137-170. Publish or Perish, Houston, TX, 1993.

[FR97] Vladimir V Fock and A A Rosly. Moduli space of flat connections as a Poisson manifold. Internat. J. Modern Phys. B, 11(26-27):31953206, 1997.

[FR99] Vladimir V Fock and A A Rosly. Poisson structure on moduli of flat connections on Riemann surfaces and the $R$-matrix. In Moscow Seminar in Mathematical Physics, volume 191 of AMS Transl. Ser. 2, pages 67-86. Amer. Math. Soc., 1999.

[Fre95] Daniel S. Freed. Classical Chern-Simons theory. I. Adv. Math., 113(2):237-303, 1995.

[Fre09] Daniel S. Freed. Remarks on Chern-Simons theory. Bull. Amer. Math. Soc. (N.S.), 46(2):221-254, 2009. 
[GM87] W. M. Goldman and J. J. Millson. Local rigidity of discrete groups acting on complex hyperbolic space. Invent. Math., 88(3):495-520, 1987.

[Gol84] William M Goldman. The symplectic nature of fundamental groups of surfaces. Adv. Math., 54(2):200-225, 1984.

[Gol86] William M Goldman. Invariant functions on Lie groups and hamiltonian flows of surface group representations. Invent. Math., 85(2):263-302, 1986.

[Gol88] William M Goldman. Topological components of the space of representations. Invent. Math., 93(3):557-607, 1988.

[Gol97] William M. Goldman. Ergodic theory on moduli spaces. Ann. of Math. (2), 146(3):475-507, 1997.

[GPiR04] Oscar Garcia-Prada and Ignasi Mundet i Riera. Representations of the fundamental group of a closed oriented surface in $\operatorname{Sp}(4, \mathbb{R})$. Topology, 43(4):831-855, 2004.

[GW10] Olivier Guichard and Anna Wienhard. Topological invariants of Anosov representations. J. Topol., 3(3):578-642, 2010.

[Hat02] Allen Hatcher. Algebraic topology. London Math. Soc. Lecture Note Ser., pages xii+544, 2002.

[Hem76] John Hempel. 3-Manifolds. Princeton University Press, Princeton, N. J., 1976. Ann. of Math. Studies, No. 86.

[Hit92] Nigel J Hitchin. Lie groups and Teichmüller space. Topology, 31(3):449-473, 1992.

[JK95] Lisa C. Jeffrey and Frances C. Kirwan. Intersection pairings in moduli spaces of holomorphic bundles on a Riemann surface. Electron. Res. Announc. Amer. Math. Soc., 1(2):57-71 (electronic), 1995 .

[KN96] Shoshichi Kobayashi and Katsumi Nomizu. Foundations of differential geometry. Vol. I. Wiley Classics Library. John Wiley \& Sons Inc., New York, 1996. Reprint of the 1963 original, A WileyInterscience Publication. 
[Kon92] Maxim Kontsevich. Intersection theory on the moduli space of curves and the matrix Airy function. Comm. Math. Phys., 147(1):1-23, Jan 1992.

[Lab10] François Labourie. An algebra of observables for cross ratios. $C$. R. Math. Acad. Sci. Paris, 348(9-10):503-507, 2010.

[Lee09] Jeffrey M. Lee. Manifolds and differential geometry, volume 107 of Graduate Studies in Mathematics. American Mathematical Society, Providence, RI, 2009.

[Li93] Jun Li. The space of surface group representations. Manuscripta Math., 78(3):223-243, 1993.

[LM85] Alexander Lubotzky and Andy R. Magid. Varieties of representations of finitely generated groups. Mem. Amer. Math. Soc., 58(336):xi+117, 1985.

[LM09] François Labourie and Gregory McShane. Cross ratios and identities for higher Teichmüller-Thurston theory. Duke Math. J., 148(9):279-345, Nov 2009.

[LP09] Sean Lawton and Elisha Peterson. Spin networks and $\operatorname{SL}(2, \mathbb{C})$ character varieties. In Handbook of Teichmüller theory. Vol. II, volume 13 of IRMA Lect. Math. Theor. Phys., pages 685-730. Eur. Math. Soc., Zürich, 2009.

[Mas91] William S. Massey. A basic course in algebraic topology, volume 127 of Graduate Texts in Mathematics. Springer-Verlag, New York, 1991.

[Mil58] John Milnor. On the existence of a connection with curvature zero. Comment. Math. Helv., 32:215-223, 1958.

[Mil97] John W. Milnor. Topology from the differentiable viewpoint. Princeton Landmarks in Mathematics. Princeton University Press, Princeton, NJ, 1997. Based on notes by David W. Weaver, Revised reprint of the 1965 original.

[Mir07a] Maryam Mirzakhani. Simple geodesics and Weil-Petersson volumes of moduli spaces of bordered Riemann surfaces. Invent. Math., 167(1):179-222, 2007. 
[Mir07b] Maryam Mirzakhani. Weil-Petersson volumes and intersection theory on the moduli space of curves. J. Amer. Math. Soc., 20(1):1-23 (electronic), 2007.

[MS74] John W. Milnor and James D. Stasheff. Characteristic classes. Princeton University Press, Princeton, N. J., 1974. Annals of Mathematics Studies, No. 76.

[Mül78] Werner Müller. Analytic torsion and $r$-torsion of Riemannian manifolds. Adv. Math., 28(3):233-305, 1978.

[NS65] M. S. Narasimhan and C. S. Seshadri. Stable and unitary vector bundles on a compact Riemann surface. Ann. of Math. (2), 82:540$567,1965$.

[Pen92] R. C Penner. Weil-petersson volumes. J. Differential Geom, Jan 1992.

[Qui85] D. Quillen. Determinants of Cauchy-Riemann operators on Riemann surfaces. Funktsional. Anal. i Prilozhen., 19(1):37-41, 96, 1985 .

[Ram75] A. Ramanathan. Stable principal bundles on a compact Riemann surface. Math. Ann., 213:129-152, 1975.

[RBKC96] A. S. Rapinchuk, V. V. Benyash-Krivetz, and V. I. Chernousov. Representation varieties of the fundamental groups of compact orientable surfaces. Israel J. Math., 93:29-71, 1996.

[Rei35] Kurt Reidemeister. Homotopie und linsenraüme. Hamburger Abhandlungen, 11:102-109, 1935.

[Ros63] Maxwell Rosenlicht. A remark on quotient spaces. An. Acad. Brasil. Ci., 35:487-489, 1963.

[RS71] D. B Ray and I. M Singer. R-torsion and the Laplacian on Riemannian manifolds. Adv. Math., 7:145-210, 1971.

[Spi79a] Michael Spivak. A comprehensive introduction to differential geometry. Vol. II. Publish or Perish Inc., Wilmington, Del., second edition, 1979.

[Spi79b] Michael Spivak. A comprehensive introduction to differential geometry. Vol. III. Publish or Perish Inc., Wilmington, Del., second edition, 1979. 
[Spr57] George Springer. Introduction to Riemann surfaces. AddisonWesley Publishing Company, Inc., Reading, Mass., 1957.

[Sti92] John Stillwell. Geometry of surfaces. Universitext. Springer-Verlag, New York, 1992. Corrected reprint of the 1992 original.

[Tur84] Vladimir G Turaev. A cocycle of the symplectic first chern class and maslov indices. Funktsional. Anal. i Prilozhen., 18(1):43-48, 1984 .

[Tur91] Vladimir G Turaev. Skein quantization of Poisson algebras of loops on surfaces. Ann. Sci. Ecole Norm. Sup. (4), 24(6):635-704, 1991.

[Wal92] Kevin Walker. An extension of Casson's invariant, volume 126 of Annals of Mathematics Studies. Princeton University Press, Princeton, NJ, 1992.

[Wit88] Edward Witten. Topological quantum field theory. Comm. Math. Phys., 117(3):353-386, 1988.

[Wit89] Edward Witten. Quantum field theory and the Jones polynomial. Comm. Math. Phys., 121(3):351-399, 1989.

[Wit91] Edward Witten. On quantum gauge theories in two dimensions. Comm. Math. Phys., 141(1):153-209, 1991.

[Wol83] Scott A Wolpert. On the symplectic geometry of deformations of a hyperbolic surface. Ann. of Math. (2), 117(2):207-234, Dec 1983.

[Woo71] John W. Wood. Bundles with totally disconnected structure group. Comment. Math. Helv., 46:257-273, 1971. 